\title{
HSP90-stabilized proteins as therapeutic targets in cancer
}

\author{
Dissertation \\ for the award of the degree \\ "Doctor rerum naturalium" \\ of the Georg-August-University Göttingen
}

within the doctoral program Molecular Medicine

of the Georg-August University School of Science (GAUSS)

submitted by

Luisa Klemke

from Hessisch Lichtenau, Germany

Göttingen 2021 


\section{Thesis Committee}

PD Dr. Ramona Schulz-Heddergott, Institute of Molecular Oncology, University Medical Center Göttingen (UMG)

Prof. Dr. Holger Reichardt, Institute for Cellular and Molecular Immunology, University Medical Center Göttingen (UMG)

Prof. Dr. Argyris Papantonis, Institute of Pathology, University Medical Center Göttingen (UMG)

\section{Members of the Examination Board}

$1^{\text {st }}$ Referee: PD Dr. Ramona Schulz-Heddergott, Institute of Molecular Oncology, University Medical Center Göttingen (UMG)

$2^{\text {nd }}$ Referee: Prof. Dr. Holger Reichardt, Institute for Cellular and Molecular Immunology, University Medical Center Göttingen (UMG)

\section{Further members of the Examination Board}

Prof. Dr. Argyris Papantonis, Institute of Pathology, University Medical Center Göttingen (UMG)

Prof. Dr. Heidi Hahn, Institute of Human Genetics, University Medical Center Göttingen (UMG)

Dr. Shiv Singh, Department of Gastroenterology, Gastrointestinal Oncology, and Endocrinology, University Medical Center Göttingen (UMG)

Dr. Nico Posnien, Department of Developmental Biology, Georg-August-University Göttingen

Date of oral examination: $8^{\text {th }}$ June 2021 
This thesis is dedicated to my family. 


\section{TABLE OF CONTENTS}

LIST OF FIGURES

ABBREVIATIONS. VII

1 ABSTRACT 1

2 INTRODUCTION. 2

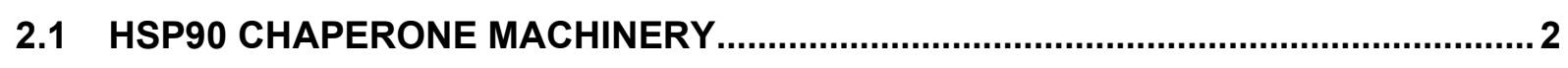

2.1.1 HSP90 CHAPERONE MACHINERY IN NORMAL CELLS ..................................... 2

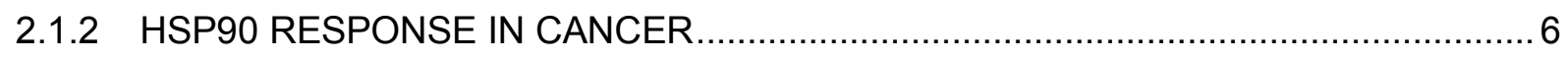

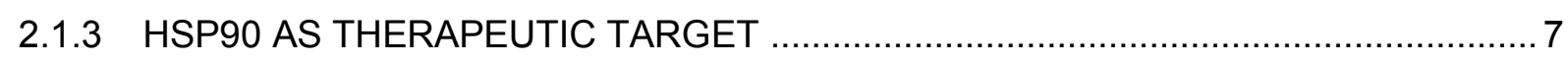

2.2 MACROPHAGE MIGRATION INHIBITORY FACTOR IN COLORECTAL CANCER.... 8

2.2.1 COLORECTAL CANCER

2.2.2 MACROPHAGE MIGRATION INHIBITORY FACTOR - A PRO-INFLAMMATORY CYTOKINE UNDER PHYSIOLOGICAL AND INFLAMMATORY CONDITIONS 10

2.2.3 MACROPHAGE MIGRATION INHIBITORY FACTOR - A TUMOR PROMOTOR UNDER ONCOGENIC CONDITIONS 12

2.3 MUTANT P53 IN PANCREATIC DUCTAL ADENOCARCINOMA ............................14

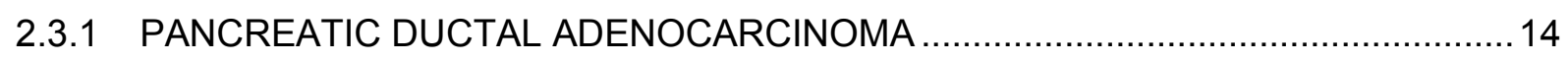

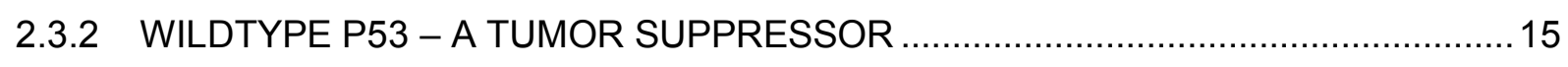

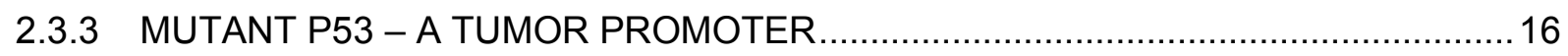

2.3.4 SPECIFIC GAIN OF NEW TUMORIGENIC FUNCTIONS BY MUTP53 ${ }^{\text {R248QNw } \ldots \ldots \ldots . . .19 ~}$

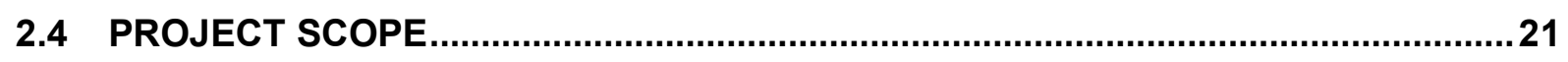

2.4.1 MACROPHAGE MIGRATION INHIBITORY FACTOR IN COLORECTAL CANCER ...21

2.4.2 MUTP53 ${ }^{\text {R248W }}$ SPECIFICITY IN PANCREATIC DUCTAL ADENOCARCINOMA .........22

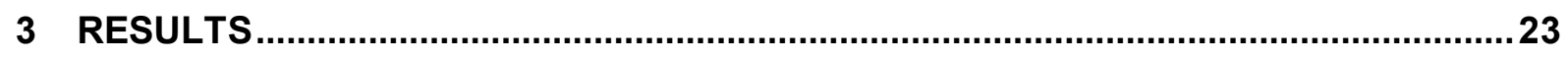

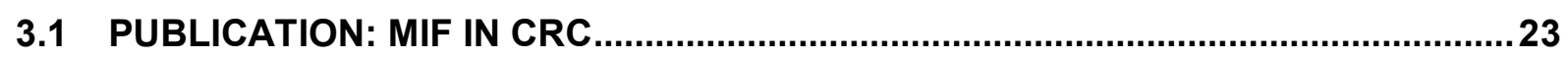

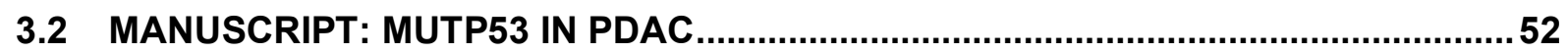


4 DISCUSSION .96

4.1 MIF PROMOTES COLORECTAL CANCER PROGRESSION .96

4.1.1 MIF CONTRIBUTES TO ANGIOGENESIS, BUT ONLY IN ESTABLISHED TUMORS A CD74-DEPENDENT MECHANISM .99

4.1.2 MIF CONTRIBUTES TO OVERALL INFLAMMATION, BUT ONLY DURING TUMOR INITIATION - A CD74-INDEPENDENT MECHANISM? . 104

4.1.3 HSP90-STABILIZED MIF CONTRIBUTES TO TUMOR CELL SURVIVAL 105

4.2 MUTP53 ${ }^{\mathrm{R} 248 / \mathrm{w}}$ PROMOTES MIGRATION IN CRC AND PDAC 108

4.2.1 DISTINCT COMPLEX FORMATION OF MUTP53 VARIANTS 110

4.2.2 STABILIZED MUTP53 AS POTENTIAL THERAPEUTIC TARGET 112

4.2.3 MUTP53 AND THE PDAC TUMOR MICROENVIRONMENT 115

4.3 CONCLUSION

5 REFERENCES $\mathbf{X I}$

6 ACKNOWLEDGEMENTS XXXIV

7 AFFIDAVIT. XXXVI

8 CURRICULUM VITAE XXXVII 


\section{LIST OF FIGURES}

Figure 1: ATPase cycle of the HSP90 chaperone machinery. ..............................

Figure 2: Transcriptional regulation of HSPs in normal cells. .................................5

Figure 3: Native forms of HSP90 in normal compared to cancer cells. .....................6

Figure 4: Pleiotropic MIF functions under physiological and inflammatory conditions.

Figure 5: MIF stabilization and functions in cancer cells. ................................13

Figure 6: Gain-of-function of mutant p53 via interaction with other proteins. ............18

Figure 7: Gain-of-function of mutp53 ${ }^{\mathrm{R} 248 \mathrm{Q} / \mathrm{W}}$ on proliferation and invasion through

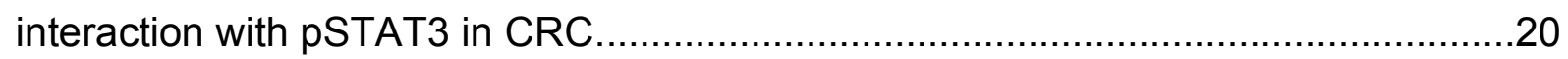

Figure 8: Functional switch of MIF during colorectal cancer progression.................98

Figure 9: Possible scenarios on how MIF triggers angiogenesis in cancer. 100

Figure 10: Migratory potential of PDAC cells depends on mutp53 ${ }^{\text {R248W }}$ and pSTAT3 complex formation 109 


\section{ABBREVIATIONS}

${ }^{\circ} \mathrm{C}$

17AAG (Tanespimycin) ADP

AHA1

AKT

AMPK

AOM

AP1

APC

ATP

BAX

CCL2

CCL5

CCND1

CD3

CD31

CD44

CD68

CD74

CDC37

CDKN1A

CDKN2A

CHIP

CHX

cJUN

Co-IP

con

CRC

cre degree celsius

17-(allylamino)geldanamycin

adenosine diphosphate

activator of $90 \mathrm{kDa}$ heat shock protein ATPase homolog 1 RAC-alpha serine/threonine-protein kinase

5'-AMP-activated protein kinase catalytic subunit alpha-1

azoxymethane

activating protein 1

adenomatosis polyposis coli

adenosine triphosphate

BCL2 associated $X$ protein

C-C motif chemokine ligand 2

C-C motif chemokine ligand 5

cyclin D1

cluster of Differentiation 3

cluster of Differentiation 31

cluster of Differentiation 44

cluster of Differentiation 68

cluster of Differentiation 74

cell division cycle 37

cyclin dependent kinase inhibitor $1 \mathrm{~A}$

cyclin dependent kinase inhibitor $2 \mathrm{~A}$

c-terminus of Hsc70-interacting protein

cycloheximide

AP1 transcription factor subunit

co-immunoprecipitation

control

colorectal cancer

cyclization recombinase 
CRISPR/Cas9

CTNNB1

CXCR2

CXCR4

CXCR7

DAPI

DBD

DMSO

DNA

DSS

ERK

ERT2

FDA

Fig

fl

GADD45A

Ganet

GC

GOF

H2AX

HER2

HHSEC

HIF1 $\alpha$

HOP

hrs

HSC70

HSE

HSF1

HSP40

HSP70

HSP90

HSR
Clustered regularly interspaced palindromic repeats/ CRISPR-associated protein 9

catenin beta-1

C-X-C motif chemokine receptor 2

C-X-C motif chemokine receptor 4

C-X-C motif chemokine receptor 7

4',6-diamidino-2-phenylindole

DNA binding domain

dimethylsulfoxide

deoxyribonucleic acid

dextran sodium sulfate

extracellular regulated MAP kinase

estrogen receptor 2

Food and Drug Administration

figure

floxed allele

growth arrest and DNA damage inducible 45 alpha

Ganetespib

glucocorticoid

gain-of-function

histone variant $2 \mathrm{~A} . \mathrm{X}$

human epidermal growth factor receptor 2

human hepatic sinusoidal endothelial cells

hypoxia inducible factor 1 subunit alpha

HSP70/HSP90 organizing protein

hours

heat shock cognate $71 \mathrm{kDa}$ protein

heat shock element

heat shock factor 1

heat shock protein 40

heat shock protein 70

heat shock protein 90

heat shock response 


\begin{tabular}{|c|c|}
\hline HUVEC & human umbilical vein endothelial cells \\
\hline IBD & inflammatory bowel disease \\
\hline IL-6 & interleukin 6 \\
\hline IL-8 (CXCL8/Kc) & Interleukin-8 \\
\hline JAB1 & JUN-activation domain-binding protein 1 \\
\hline JAK2 & janus kinase 2 \\
\hline kDa & kilodalton \\
\hline Ki67 (MKI67) & marker of proliferation Ki-67 \\
\hline KIP1/p27 (CDKN1B) & cyclin dependent kinase inhibitor $2 \mathrm{~A}$ \\
\hline KRAS & kirsten rat sarcoma viral oncogene homolog \\
\hline LOF & loss-of-function \\
\hline LOH & loss-of-heterozygosity \\
\hline MAPK & mitogen-activated protein kinase \\
\hline MDM2 & mouse double minute 2 homolog \\
\hline MIF & macrophage migration inhibitory factor \\
\hline MKP1 & mitogen-activated protein kinase phosphatase 1 \\
\hline $\mathbf{m L}$ & milliliter \\
\hline MPO & myeloperoxidase \\
\hline mRNA & messenger RNA \\
\hline mutp53 & mutant p53 \\
\hline ng & nanogramm \\
\hline $\mathrm{nM}$ & nanomolar \\
\hline NOS2 & nitric oxide synthase 2 \\
\hline NOXA/PMAIP1 & phorbol-12-myristate-13-acetate-induced protein 1 \\
\hline NP-40 & Nonidet $\circledast \mathrm{P} 40$ \\
\hline ns & not significant \\
\hline Onales & Onalespib \\
\hline OSM & oncostatin $\mathrm{M}$ \\
\hline oxMIF & oxidized MIF \\
\hline p38 MAPK & p38 mitogen-activated protein kinase \\
\hline PDAC & pancreatic ductal adenocarcinoma \\
\hline PGE2 & prostaglandin E2 \\
\hline PI3K & phosphoinositide 3 kinase \\
\hline
\end{tabular}


PLA2

pSTAT3

PTGS2/COX2

PUMA

$\mathbf{R}$

Rad21

rhMIF

RNA

scr

SDS

SHP2

SIRNA

SMAD4

SRC

TAM

TF

TNFa

TP53

TP63

TP73

Tris

VEGF

wks

WT

$\mu \mathrm{g}$

$\mu \mathrm{l}$

$\mu \mathrm{m}$

$\mu \mathrm{M}$ phospholipase A2

phosphorylated signal transducer and activator of transcription 3

prostaglandin-endoperoxide synthase 2/ cyclooxygenase 2 p53 upregulated modulator of apoptosis

pearson correlation coefficient

double-strand-break repair protein rad21 homolog

recombinant human MIF

ribonucleic acid

scrambled siRNA

sodium dodecyl sulphate

src homology 2 (SH2) domain-containing phosphatase 2

small interfering RNA

mothers against decapentaplegic homologue 4

non-receptor tyrosine kinase

tamoxifen

transcription factor

tumor necrosis factor alpha

tumor protein 53

tumor protein 63

tumor protein 73

trisamine

vascular endothelial growth factor

weeks

wildtype

microgram

microliter

micrometer

micromolar 


\section{ABSTRACT}

Although cancer is among the most common causes of death worldwide, successful treatment options for most cancer entities remain elusive, raising the need for novel therapies. One attractive target of current drug candidates is the stress-inducible heat shock protein 90 (HSP90) chaperone machinery. Its normal chaperoning function is subverted in tumors to protect numerous mutated and overexpressed proteins from misfolding and degradation. Hence, it is playing a central role in oncogenic signaling. The addiction of cancer cells to the HSP9O chaperone machinery provides opportunities for targeting the stability of HSP90-dependent oncoproteins (clients).

To dissect the importance of the HSP90 chaperone machinery in tumor progression, we investigated two HSP90-stabilized proteins: the macrophage migration inhibitory factor (MIF) in colorectal cancer (CRC) and mutant p53 (mutp53) in pancreatic ductal adenocarcinoma (PDAC). Both proteins were shown to be elevated in cancer cells via the HSP90 chaperone machinery, correlating with worse prognosis for cancer patients. MIF is a pro-inflammatory cytokine which is known to promote tumor progression in various cancer entities. Indeed, we demonstrate that loss of HSP90-stabilized MIF in CRC results in reduced tumor growth. This effect was accompanied by decreased macrophage recruitment and angiogenesis in established CRC tumors. Our data suggest that MIF acts via the CD74/MAPK axis and is indeed a cancer-relevant HSP90 client in CRC.

The tumor suppressor p53 (p53) is mutated in approximately $50 \%$ of all human cancers. We found that the mutp53 ${ }^{\mathrm{R} 248 \mathrm{~W}}$ variant is highly stabilized by the HSP90 chaperone machinery in pancreatic ductal adenocarcinoma (PDAC) cells. Furthermore, we identified a unique gain-of-function role of this $p 53^{\mathrm{R} 248 \mathrm{~W}}$ mutant on cell migration. Mechanistically, mutp53 ${ }^{\mathrm{R} 248 \mathrm{~W}}$ specifically interacts with the phosphorylated transcription factor STAT3 and thus contributes to the aggressiveness of pancreatic cancer.

Our results further corroborate HSP90 as an attractive target to counteract tumor development, and we identified two HSP90 clients as cancer drivers, outlining additional target structures for cancer therapy. 


\section{INTRODUCTION}

Cancer is one of the leading causes of death in the world, with rising incidence every year [1, 2]. It is considered a 'disease of change', marked by broad genetic and phenotypic heterogeneity and plasticity [3]. Remarkable efforts and advances have been made trying to understand the complexity of this disease. However, clinical trials for cancer therapies are the least successful compared to major other diseases $[4,5]$. To address this issue, researchers from all over the world are focusing on targeted therapy and personalized oncology in order to develop tailor-made and specialized treatments for cancer patients [3,5]. In this respect, it is particularly important to identify cancer-relevant biomarkers to increase the success rates of clinical trials $[4,5]$. Several driver mutations have been identified to be essential for tumor initiation, providing a selection advantage for mutated cells [6-8]. However, throughout tumor development, cancer cells acquire genetic and epigenetic mutations as well as molecular alterations $[9,10]$. Together with environmental factors such as nutrient/oxygen starvation and oxidative stress, these mutations and alterations induce different stress responses in cancer cells [11-15]. One of these responses is the heat shock response (HSR), leading to the induction of stress proteins such as heat shock protein 90 (HSP90) [1416]. By assisting in the stabilization and activation of many proteins (termed clients), in particular oncogenes, HSP90 constitutes a suitable target for cancer therapy [16, 17]. Given the plethora of stabilized oncogenes, HSP90 inhibitors provide a possibility to overcome resistance mechanisms of cancer cells towards conventional chemotherapy [18-21].

\subsection{HSP9O CHAPERONE MACHINERY}

\subsubsection{HSP90 chaperone machinery in normal cells}

Heat shock proteins (HSPs) are a highly conserved ubiquitous family [16, 22-24]. Since their first discovery in 1962 [25], these proteins have been extensively studied and classified according to their molecular weights [26, 27]. Through assisting in the regulation of turnover, cellular localization and trafficking as well as activity of various proteins, HSPs can regulate growth, survival and differentiation of cells [16, 23, 28-30]. 
One of the most abundant stress proteins is the molecular chaperone HSP90 [24, 31, 32]. For HSP90, two major cytoplasmic isoforms have been identified: the inducible HSP90 $\alpha$ isoform and the constitutive HSP90 $\beta$ isoform [24, 33]. HSP90 predominantly exists as homodimer of either isoform; however, monomers and heterodimers have also been reported [24, 33, 34]. Both isoforms consist of a C-terminal domain as well as a middle and $\mathrm{N}$-terminal domain which are connected via a charged linker [23, 33]. The C-terminal domain is necessary for the interaction of two HSP90 monomers in order to form a functional dimer [35-37]. In contrast, the $\mathrm{N}$-terminal domain is required for binding and hydrolysis of ATP molecules [35]. Importantly, assembly with other cochaperones is necessary to form the full functional HSP90 chaperone machinery [29, 38]. An ATP-dependent chaperone cycle contains various steps of temporary and dynamic protein interactions, posttranslational modifications and conformational changes [23, 38] (Figure 1). In this manner, HSP90 can affect structure and functionality of its client proteins [38, 39]. For proper maturation, the chaperones HSP40 and HSP70 are the first to bind to the nascent polypeptide chain, forming the early complex [23, 39, 40]. HSP70 of the early complex can bind to HSP90 via the adaptor protein HOP (HSP70/HSP90 organizing protein), allowing the client to be transferred to HSP90 (intermediate complex) [23, 39-43]. If a polypeptide chain cannot be formed properly, the presence of co-chaperones such as the E3 ubiquitin-protein ligase CHIP (C-terminus of Hsc70-interacting protein) target the client peptide for proteasomal degradation [43, 44]. In contrast, proper maturation of clients are achieved by binding of ATP to HSP90, resulting in a transition into a 'closed and twisted' conformation, which is characterized by the interaction of the middle and $\mathrm{N}$ terminal domains [45]. At this stage, binding of the other co-chaperones triggers the dislocation of HOP, HSP70 and HSP40 to form the late complex [29, 39]. After hydrolysis of ATP, the mature protein is released. Dissociation of ADP reinstates the initial open conformation of HSP90, thus allowing binding of new clients [38, 39]. 


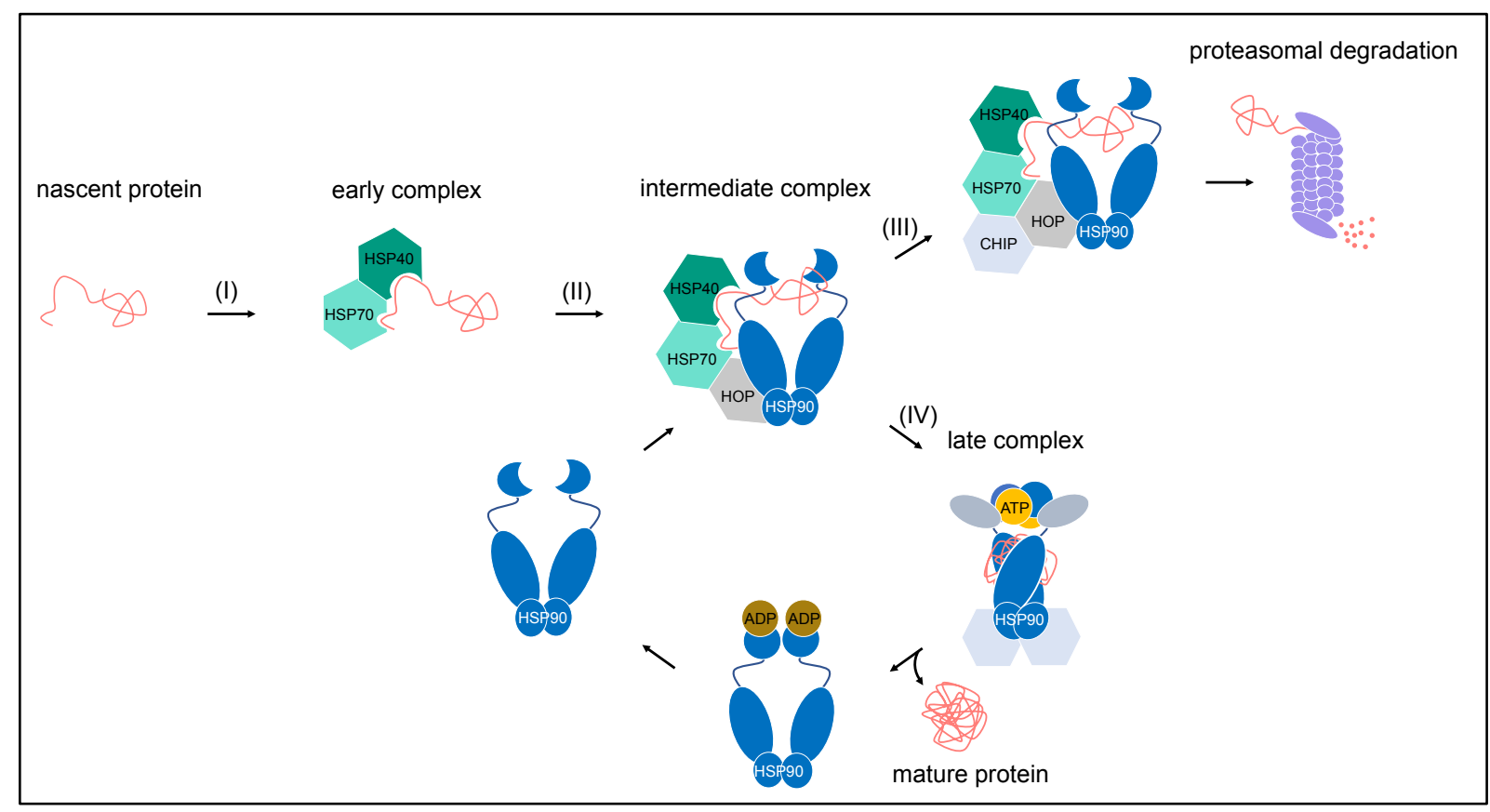

Figure 1: ATPase cycle of the HSP90 chaperone machinery. (I) Nascent polypeptide chains are being captured by HSP90 co-chaperones HSP70, HSP40 to form the early complex. (II) Binding of HSP70 to HSP90 via HOP allows translocation of the nascent polypeptide chain from the early complex to the HSP90 chaperone (intermediate complex). (III) Binding of the co-chaperone CHIP or other E3 ubiquitin protein ligases mark nascent proteins for proteasomal degradation. (IV) In contrast, binding of ATP to the N-terminal domain of HSP90, results in its transition into the 'closed and twisted' conformation, which is characterized by interaction of its middle and $\mathrm{N}$-terminal domains. Other cochaperones displace HSP70/HSP40 and HOP and assist in stabilizing the conformation of the late complex. After hydrolysis of ATP, the mature protein is released, and dissociation of ADP reverts HSP90 into its initial open conformation.

To this date, the mechanism behind how HPS90 recognizes its client proteins remains elusive, since no common patterns have been discovered thus far [23]. In the past decades, more than 20 co-chaperones have been identified, exhibiting different molecular functions [23, 39]. Additionally, a variety of post-translational modifications of HSP90 such as acetylation, phosphorylation and nitrosylation are known to regulate the activity of the protein and modulate its specificity to other co-chaperones or clients $[17,23,46]$. Therefore, the exact composition of co-chaperones and the dynamics of the ATPase cycle highly depend on the molecular context, the presence of posttranslational modifications and the client to be processed [23, 45, 47, 48].

Among the main transcriptional regulators of HSPs are the members of the heat shock factor (HSF) family [49]. In vertebrates, the most important regulator of HSPs is the heat shock factor 1 (HSF1) [49, 50]. Under physiological conditions, inactive 
monomeric HSF1 is bound to HSP90 [51-54]. However, cellular stress can trigger the accumulation of unfolded proteins which activates HSP90 [23, 51]. Unfolded proteins are captured by HSP70 and HSP40, for further translocation of the unfolded protein to HSP90 [42, 51]. The unfolded or misfolded proteins are further processed as described more detailed in section 2.1.1 to form a mature protein. This process leads to the displacement of HSF1 from HSP90, allowing the formation of an active trimeric HSF1 complex [49, 53] (Figure 2). The trimeric HSF1 molecule translocates to the nucleus to act as a transcription factor $[49,51]$. It binds to heat shock elements (HSE), a promotor region upstream of the HSP genes to initiate the expression of heat shock proteins such as HSP90 and HSP70 [49, 50].

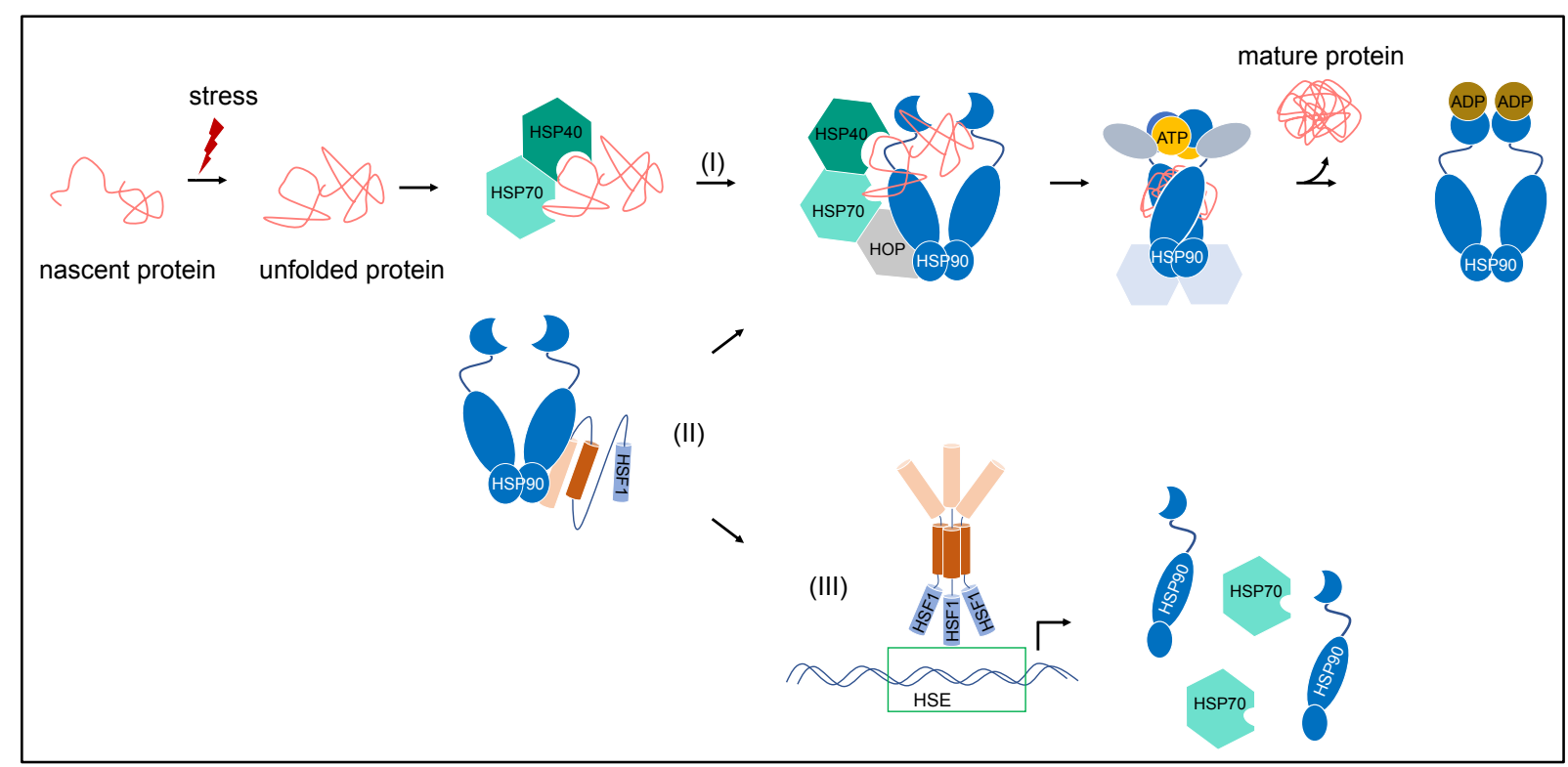

Figure 2: Transcriptional regulation of HSPs in normal cells. Nascent polypeptide chains accumulate to unfolded or misfolded proteins due to cellular stress. (I) HSP40 and HSP70 capture these unfolded proteins for translocation to HSP90. (II) Under physiological conditions, inactive monomeric HSF1 is bound to HSP90. Under stressed conditions, HSF1 dissociates from HSP90, resulting in its trimerization and transcriptional activation. (III) In the nucleus, HSF1 binds to heat shock elements (HSE) upstream of the heat shock protein genes such as HSP9O and HSP7O.

According to the updated list by the Picard Lab, more than $800 \mathrm{HSP} 90$ client proteins have been identified thus far [21]. Given the plethora of clients, it is not surprising that heat shock proteins can contribute to the development and progression of many pathological conditions such as cancer, neurodegenerative or infectious diseases [30, 38, 42, 43, 55]. Two HSP90-stabilized clients which have been associated with tumor progression are the macrophage migration inhibitory factor (MIF) and mutant p53 (mutp53) [56, 57]. 


\subsubsection{HSP9O response in cancer}

The elevated expression of HSP90 and other co-chaperones have been reported previously for many different cancer entities $[17,58]$, such as breast [59,60], colorectal $[61,62]$ or pancreatic cancers $[63,64]$. In most cases, the overexpression of chaperones in general and HSP90 in particular, correlates with decreased survival of cancer patients [59, 61, 62]. Cancer cells are rapidly proliferating cells, with a high demand for newly synthesized proteins $[13,15]$. The extensive amount of cytotoxic stress, caused by the high mutational load, oxygen and nutrient starvation, can give rise to an accumulation of unfolded or misfolded proteins [15, 16, 23, 51, 65]. In order to cope with this cellular stress, which could be detrimental and cytotoxic in the long run, cancer cells respond by inducing HSP expression [16, 66] (Figure 2). While HSF1 and HSP90 strongly co-regulate each other in normal cells [49], constant cellular stress in cancer cells results in a constitutive activation of HSF1 and HSF1-mediated chaperone expression, which can favor the formation of superchaperone complexes [67-71] (Figure 3).

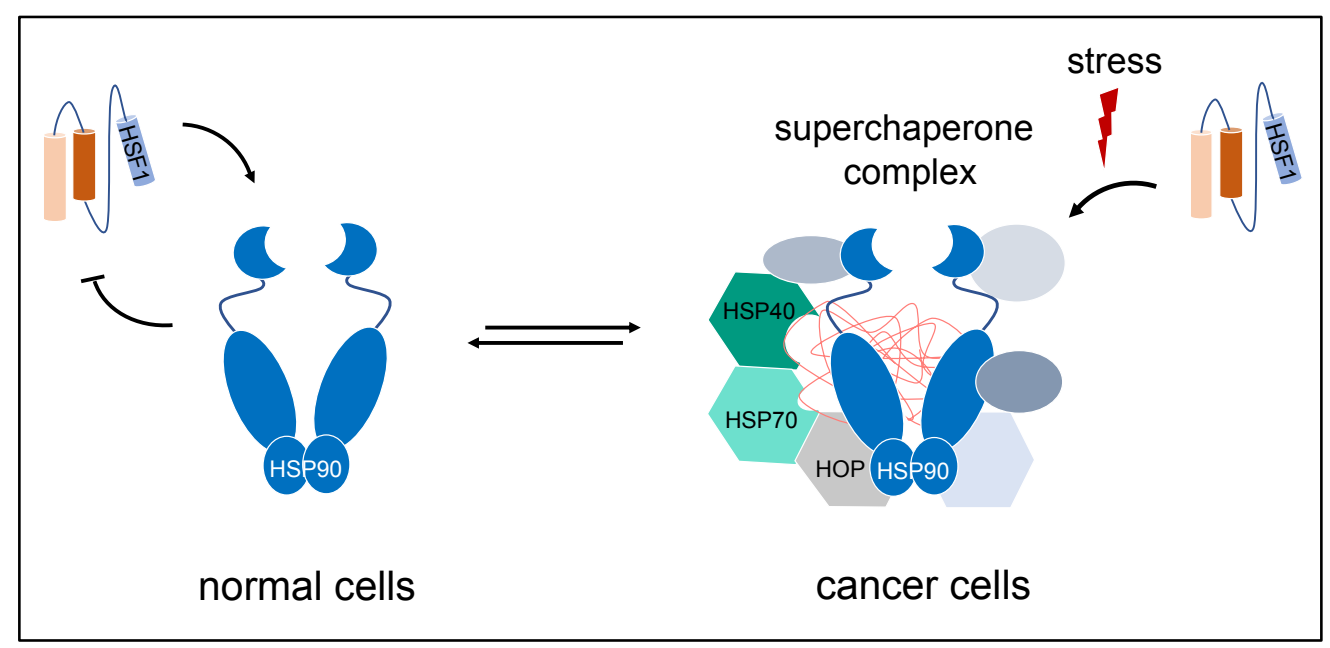

Figure 3: Native forms of HSP90 in normal compared to cancer cells. In normal cells, HSP90 exists as a transient dimer in order to assist in protein turnover and activity. Cellular homeostasis is achieved by counter regulation between HSF1 and HSP90. Due to high stress levels in cancer cells, HSF1 is constitutively active, resulting in increased expression of HSP90 and other chaperones. The association of multiple co-chaperones with HSP90 leads to the formation of superchaperone complexes, which in turn support increased stabilization and decreased degradation of its client proteins.

These superchaperone complexes further support tumor development by assisting in the proper maturation, stabilization and activity of a plethora of oncogenes (such as mutp53, AKT, v-SRC) $[16,56,71,72]$. Thus, HSP90 helps to fulfill the hallmarks of 
cancer defined by Hanahan and Weinberg, which includes increased proliferation, angiogenesis, invasion and metastasis [17, 73, 74].

In that context, several cancer-relevant HSP90 clients have already been identified across different cancer origins [72, 75]. In breast cancer for example, the human epidermal growth factor receptor-2 (HER2; ErbB2/Neu) was described as an important client of the HSP90 chaperone machinery [72, 76, 77]. Interestingly, a previous publication from our group found that HER2 overexpression regulates hyperactivation of HSF1, resulting in increased induction of the HSP90 chaperone machinery (Figure 2) and thus further stabilization of HSP90 clients, providing a positive feed-forward loop [76]. In comparison, the androgen receptor was identified as important HSP90 client relevant for prostate cancer progression $[75,78]$.

Because of its pleiotropic functions, the abundance of clients and large impact on tumor development and progression, HSP90 is considered a promising therapeutic target in cancer therapy $[16,72,75]$.

\subsubsection{HSP90 as therapeutic target}

The aberrant activation of HSP90 within the superchaperone complexes results in higher ATPase activity of HSP90 in cancer cells compared to normal cells [68, 71, 79]. Consequently, HSP90 is considered a potential target to selectively affect cancer cells $[71,80]$. In the past decades, several clinical trials have been performed using small molecules, which are able to bind and inhibit the N-terminal ATP binding pocket of HSP90, resulting in client degradation of cells with high ATPase activity such as cancer cells [71, 79, 81]. Unfortunately, first-generation inhibitors such as Tanespimycin (17AGG) showed low efficacy, high toxicity and reduced solubility [82, 83]. In order to address this issue, second-generation inhibitors such as Ganetespib (STA9090) and Onalespib (AT13387) have been developed and applied to clinical trials [20, 82, 84]. Using these inhibitors, several in vivo studies confirmed decreased tumor development for T-cell lymphomas [85], non-small cell lung cancers [86, 87] and breast cancers [88]. However, other studies in cancer entities such as colorectal cancer demonstrated low antitumor efficacy of HSP90 inhibition alone, with efficacy only observed in the presence of other chemotherapeutics $[89,90]$. 
Even though second-generation inhibitors have shown lower cytotoxicity compared to first-generation inhibitors [20,83], only limited efficacies have been reported in some in vivo studies and clinical trials $[20,84,89,90]$. So far, none of the 18 HSP90 inhibitors in development have shown sufficient efficacy for FDA (Food and Drug Administration) approval $[14,89,91]$. The limited efficacy of these HSP90 inhibitors was thought to be caused by the activation of HSF1 [84, 92]. The HSF1 mediated heat shock response (HSR) results in increased expression and activation of HSPs such as HSP70 (Figure 2) which can diminish the effects of an HSP90 inhibitor [49, 92]. In order to reduce the HSR, inhibitors binding to the C-terminal domain or those that disrupt the HSP9O protein-protein interactions are currently investigated [67, 92-95]. Such inhibitors have shown encouraging results thus far, causing the degradation of client proteins with limited induction of the HSF1 response compared to the N-terminal inhibitors [67, 92, 94].

Taken together these results have further emphasized the need to investigate the molecular basis of the disease for targeted and personalized medicine and the need for predictive cancer-relevant biomarkers for HSP90 inhibitors. Because of that, we are investigating the role of two important HSP90 clients: the macrophage migration inhibitory factor (MIF) and mutant p53 (mutp53).

\subsection{MACROPHAGE MIGRATION INHIBITORY FACTOR IN COLORECTAL CANCER}

\subsubsection{Colorectal Cancer}

Colorectal cancer (CRC) is the third most common cancer worldwide, with rising incidence every year [96-98]. In the majority of cases, the disease is caused by sporadic mutations due to environmental factors like age or lifestyle factors such as improper diet and the lack of physical exercise [96, 99]. However, a minority of CRC cases occur as a result of inherited genetic mutations [96, 99]. As early as in the late 1980s, Vogelstein and colleagues demonstrated, that a series of oncogene mutations and loss of tumor suppressors are essential for the development and progression of sporadic CRC [100, 101]. Genetic alterations of APC (adenomatosis polyposis coli), CTNNB1 (catenin beta-1), KRAS (Kirsten ras oncogene homolog), TP53 (tumor protein 53), and SMAD4 (mothers against decapentaplegic homologue 4) are essential 
drivers for the successive malignant degeneration of normal mucosa to carcinoma and metastasis [96, 99, 101]. Other important causative factors for colorectal cancer are inflammatory bowel diseases (IBD) such as ulcerative colitis and crohn's disease [96, 99, 102]. Due to the characteristic chronic and relapsing inflammation of these diseases, leading to inflammation-induced damage of the intestinal tissues, IBD patients are highly susceptible to developing CRC $[99,102]$.

To investigate a colitis-associated type of colorectal cancer, the AOM/DSS mouse model can be used [103-105]. This model entails a single injection of azoxymethane (AOM), a carcinogenic chemical agent causing DNA damage in the colon due to methylation of guanosine [103, 104]. After one week of rest, dextran sodium sulfate (DSS) is added to the drinking water, causing an acute inflammation in the intestine, due to the disruption of the epithelial barrier and infiltration of the microbiome into the tissue $[103,106]$. After administration of both agents, rodents develop tumors in the colorectal part of the intestine, mimicking the human patient situation $[103,105]$.

In sum, inflammation can play a crucial role in the development of colorectal cancer. Due to the long symptom-free tumor growth and the broad range of occurring symptoms, most patients are diagnosed at later stages of the disease $[107,108]$. Thus, preventive CRC screenings as well as development of predictive biomarkers are essential to allow earlier detection and better prognosis of these tumors [102, 107, 108].

It has been shown in various human cancer entities that elevated MIF levels in epithelial tumor cells correlated with poorer patient prognosis [109-112]. In a mouse model for breast cancer, we previously showed that elevated MIF levels are due to a stabilization through the HSP90 chaperone machinery, thus contributing to tumor progression [57, 80]. Hence, in the current study we investigated whether MIF is a tumor driver and can serve as potential drug target in CRC.

Before describing the role of MIF in cancer cells, the next section depicts the functions of the pro-inflammatory cytokine under physiological and inflammatory conditions. 


\subsubsection{Macrophage migration inhibitory factor - a pro-inflammatory cytokine under physiological and inflammatory conditions}

Macrophage migration inhibitory factor (MIF) is a ubiquitous pro-inflammatory cytokine involved in inflammatory and immune responses [113-115]. First discovered in 1966, it was shown to inhibit random migration of peritoneal macrophages [116-118] and to function as a homotrimer $[119,120]$. Besides its function as a pro-inflammatory cytokine, it was also reported to have enzymatic activity via its tautomerase domain [119-121]. MIF was shown to be involved in a plethora of disorders such as cardiovascular [122-124], neurodegenerative [125] and pulmonary diseases [126].

MIF can fulfil its pleiotropic functions either via receptor-mediated pathways by binding to CD74/CD44, CXCR2, CXCR4 and CXCR7 [113, 123, 127-130] or through receptordependent or independent endocytosis [113, 124, 131, 132] (Figure 4). By binding to transmembrane receptors extracellular MIF initiates various downstream signaling cascades and assists in diverse cellular functions $[113,123]$. Depending on the cellular context, these receptors function individually or as heterocomplexes [124, 129, 130]. The exact signaling pathway for MIF highly depends on the cellular context, the expression of the receptors on different cell types as well as environmental factors such as the expression of ligands that compete with MIF for binding to non-cognate receptors [128-130]. Binding of MIF to the non-cognate receptors CXCR2 or CXCR4 actives G-proteins, whereas interaction with CXCR7 activates ß-arrestin which inhibits short term G-protein coupled receptor signaling and results in a long-lasting ERK1/2 (extracellular regulated MAP kinase) and PI3K (phosphoinositide 3 kinase) activation $[130,133]$. Binding to the cognate CD74 receptor triggers its dimerization with CD44 and the activation of a downstream signaling cascade, for example via the tyrosine kinase SRC $[127,134]$. The individual and combined activation of these pathways can result in the activation of MAP-kinases or PI3K/AKT, that results in cell proliferation, migration and angiogenesis $[113,124,128,129,133,134]$. Furthermore, activation of ERK1/2 promotes induction of phospholipase A2 (PLA2) and cyclooxygenase 2 (COX2) resulting in transformation of arachidonic acid to prostaglandin $\mathrm{E}_{2}\left(\mathrm{PGE}_{2}\right)$, an essential driver of inflammatory responses [113, 135-137]. Simultaneously, MIFmediated activation of COX2 is suggested to have an inhibitory impact on p53, resulting in decreased apoptosis and further supporting cellular survival and proliferation $[113,133,138]$. However, MIF can also enter cells via endocytosis [113, 
124]. Intracellular MIF can for instance bind to JAB1 (JUN-activation domain-binding protein 1) resulting in its inactivation $[139,140]$. The inactivation of JAB1 prevents activation of cJUN (AP1 transcription factor subunit), which functions as a co-activator of the activator protein 1 (AP1), known to be involved in the regulation of proliferative and inflammatory signals [113, 139-141]. Furthermore, MIF-JAB1 diminishes JAB1 induced degradation of the cyclin-dependent kinase inhibitor KIP1 (p27), resulting in increased cell cycle arrest $[113,139,142,143]$. In this respect, high levels of intracellular MIF can counteract MIF-receptor induced pathways [122, 124].

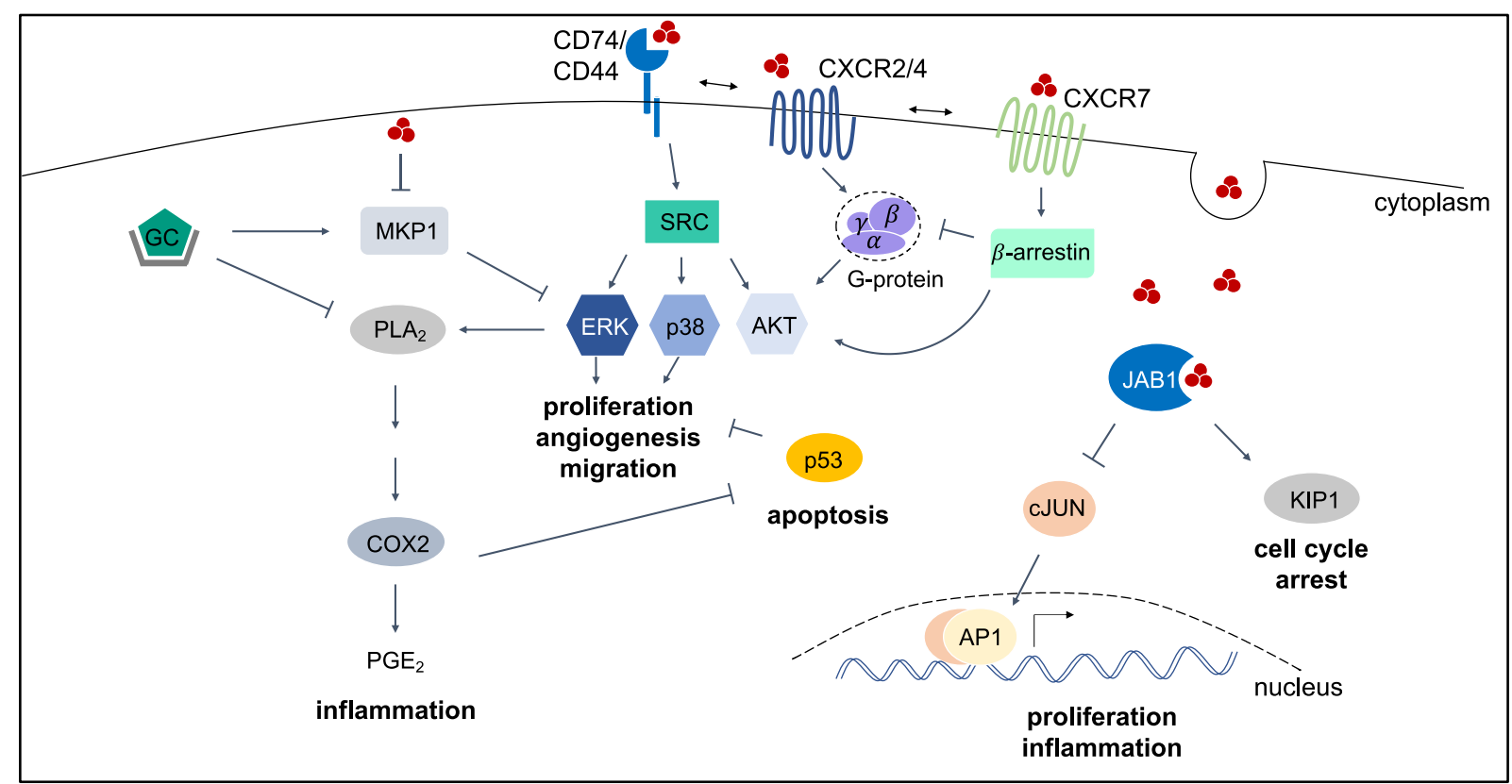

Figure 4: Pleiotropic MIF functions under physiological and inflammatory conditions. Macrophage migration inhibitory factor (MIF) acts in a receptor-dependent manner by binding to CD74/CD44, CXCR2/4/7 or can enter cells via endocytosis. Depending on the molecular context, the different MIF receptors can form heterocomplexes, which trigger downstream activation of MAPKs ERK1/2, p38 or PI3K/AKT supporting migration, proliferation, angiogenesis and inflammation of cells. MIF-induced activation of COX2 can have an inhibitory impact on p53, further promoting cellular survival by inhibiting apoptotic responses. High intracellular levels of MIF can counteract the receptorinduces pathways, caused by MIF-mediated inhibition of JAB1. This subsequently, leads to an induction of cell cycle arrest (via KIP1) and a reduction of inflammatory and proliferative signals (via cJun/AP1).

Counter regulatory activity has been reported between MIF and glucocorticoids (GC), hormones which are important anti-inflammatory players [113, 122, 144]. By inhibiting $\mathrm{PLA}_{2}$ or inducing the expression of mitogen-activated protein kinase phosphatase 1 (MKP1, the main negative regulator of ERK), high levels of glucocorticoids, can hamper MIF-induced pathways [137]. Similarly, it has been reported that MIF is able to counteract glucocorticoids for example through inhibition of MKP1 [113, 137, 144]. 
Therefore, a balance between the pro-inflammatory cytokine MIF and the antiinflammatory glucocorticoids is essential for proper cellular homeostasis in order to prevent the development of diseases $[122,130]$.

Taken together, there is an increasing amount of evidence over a number of years, that MIF is not just a pro-inflammatory cytokine involved in inflammatory responses but is also a potential driver of tumor development $[145,146]$.

\subsubsection{Macrophage migration inhibitory factor - a tumor promotor under oncogenic conditions}

It has been shown in various human cancer entities such as breast [109], prostate $[110,147]$, colon [111, 148] and hepatocellular carcinomas [112] that elevated MIF levels in epithelial tumor correlated with poorer patient prognosis.

Thus far, the role of MIF in intestinal cancer has been investigated in vivo by using $A p c^{\text {min }}$ mice [149] or xenograft tumor models [111, 150,151], confirming the tumor supportive role of MIF is this cancer entity. However, a causative in vivo model, mimicking the human patient situation remains elusive, since patients with malignant intestinal neoplasia mostly develop tumors in the distal part of the intestine (colon and rectum) [105]. Only a minority of tumors grow in the small intestine, as observed for the $A p c^{\min }$ mice [152]. Therefore, we have chosen to investigate the role of MIF as cancer-relevant HSP90 client in a more clinically relevant approach using the AOM/DSS mouse model to induce colitis-associated cancer [103].

In a recent study, our research group was able to show that MIF levels are elevated in breast cancer cells [57]. These high MIF levels (Figure 5) arise as a consequence of cellular stress induced activation of HSF1, triggering the expression of various chaperones [49, 57, 76, 80] (Figure 2). As a consequence, the formation of superchaperone complexes (Figure 3 ) leads to high stabilization of the MIF protein $[57,67,76]$. Furthermore, oxygen starvation in cancer cells triggers the activation of hypoxia inducible factor 1 alpha (HIF1 $\alpha$ ), a major transcription factor of MIF and proangiogenic genes such as VEGF [153-156]. In addition, extracellular MIF can bind to its receptors in an autocrine or paracrine fashion in order to trigger proliferation, angiogenesis, migration or apoptosis of cells via induction of the MAP kinases ( $p 38$, ERK1/2) or PI3K/AKT [111, 150, 151, 157-162]. In a positive feed-forward loop, these 
proteins can further contribute to the activation of HSF1 and HIF1 $\alpha$ to trigger MIF expression and stabilization [70, 76, 155, 163, 164]. In cancer cells, MIF can also contribute to inflammatory processes by activation of $\mathrm{PLA}_{2}$ [159]. The inhibitory impact of COX2 on the tumor suppressor p53 contributes to decreased apoptosis, which further supports cancer cell survival and proliferation [138, 159, 165, 166].

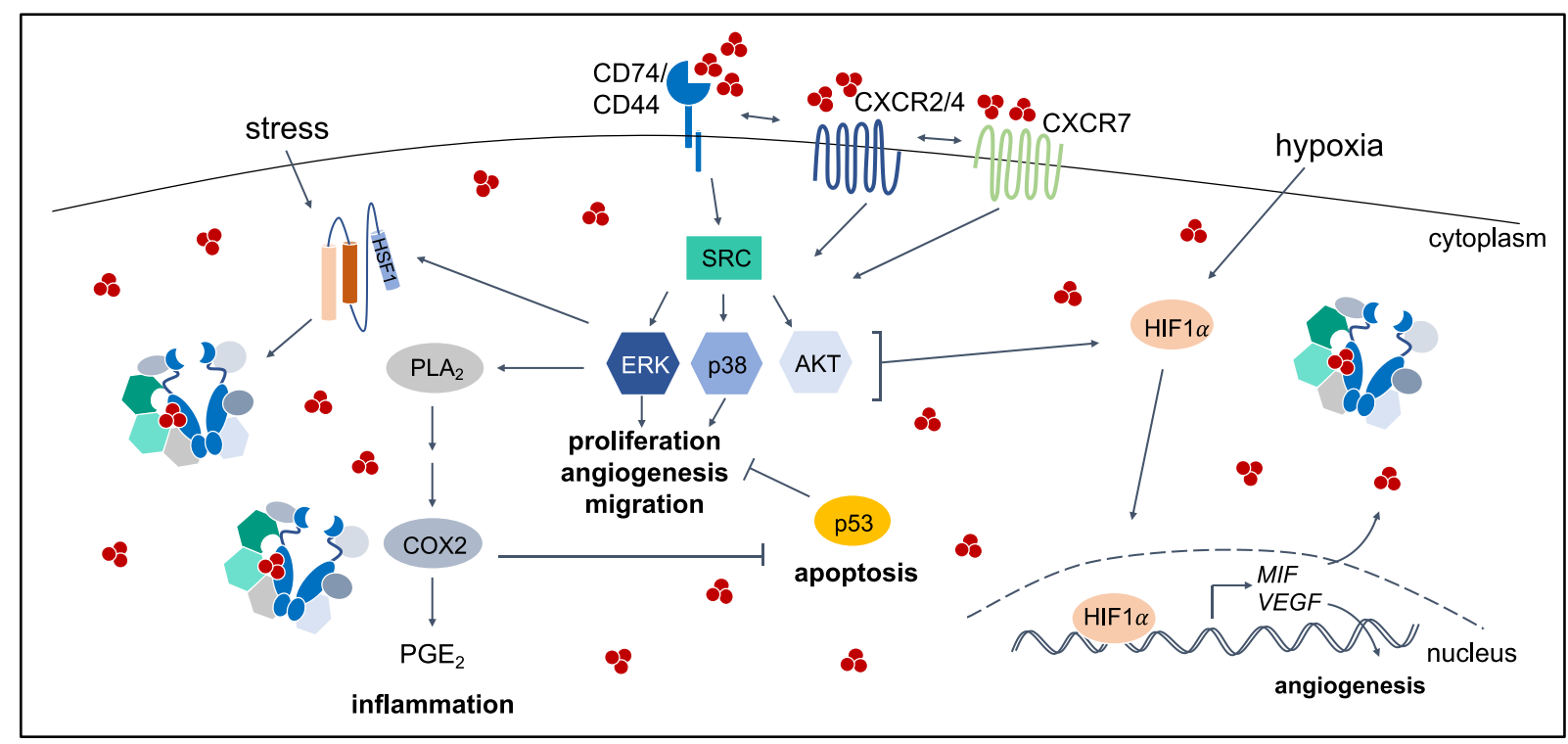

Figure 5: MIF stabilization and functions in cancer cells. Stabilization of MIF via HSP90 multichaperone complexes results in elevated MIF protein levels in cancer cells and serum. Furthermore, constant cellular stress and oxygen starvation induce constitutive activation of HIF $1 \alpha$ and HSF1. HSF1 further promotes activation and expression of the HSP90 chaperone machinery. HIF1 $\alpha$ can function as a transcription factor for MIF further promoting elevation of MIF levels on gene expression levels in cancer cells. Increased binding of MIF to its receptors enhances activation of downstream signaling pathways to promote proliferation, migration and inflammation to promote carcinogenesis.

Notably, the increased expression, stabilization and secretion of MIF from cancer cells and its chemokine like properties, can further contribute to the recruitment and activation of tumor promoting immune cells such as tumor associated macrophages $[123,154,155,167]$. Constituents of the tumor microenvironment can support tumor progression, not just by expressing and secreting MIF themselves [148, 168], but also by producing and secreting various cytokines which can further promote tumorigenic proliferation and angiogenesis $[167,169,170]$.

In cancer cells, MIF has also been shown to promote cellular resistance to stress or chemotherapeutics via regulation of MAPKs, STAT3 or AMPK, subsequently 
promoting tumor cell survival $[157,171,172]$. Because of its pleiotropic functions associated with tumor progression, MIF has been considered a promising selective target for cancer therapy $[80,157,159]$. Targeting of MIF can be achieved by three main strategies:

(I) Direct binding of small molecules to the tautomerase domain, which is known to be important for the interaction with other proteins $[173,174]$.

(II) By using monoclonal antibodies against MIF or its cognate receptor CD74, resulting in a modulation of the downstream signaling cascades and interference with MIF induced tumorigenic pathways [175].

(III) Indirect inhibition through its destabilization using HSP90 inhibitors, resulting in decreased MIF levels in cancer cells and diminished MIF-induced tumorigenic functions $[57,76]$.

To exploit the potential of anti-MIF therapy in the field of oncology, a number of approaches have also been used in preclinical as well as some clinical trials supporting MIF's tumorigenic potential $[159,175]$. However, additional research is necessary to fully understand the tumor promoting mechanism of action and the potential of using MIF as a biomarker for CRC.

\subsection{MUTANT P53 IN PANCREATIC DUCTAL ADENOCARCINOMA}

\subsubsection{Pancreatic ductal adenocarcinoma}

Pancreatic cancer is the seventh leading cause of cancer death worldwide, with rising fatalities every year [2, 98]. Of all pancreatic malignancies, pancreatic ductal adenocarcinoma (PDAC) occurs with a frequency of more than $90 \%$ and is considered as highly chemoresistent and poorly treatable due to limited medical and surgical options [176-179]. The late onset of symptoms combined with a high capability to metastasize and high plasticity of the cancer cells, make PDAC one of the most aggressive entities with a five-year survival rate of around 8\% [176-178]. Due to the broad heterogeneity of PDAC, several efforts have been made in order to specify defined PDAC subtypes based on histological findings as well as transcriptome analysis. Taken together, it is suggested to distinguish between the epithelial-like 
classical subtype and the more aggressive basal-like quasi-mesenchymal subtype [177, 180-183].

PDAC derives from pancreatic intraepithelial neoplasia which are categorized into three stages (PanIN1-3) and characterized by successive accumulation of genetic mutations and increasing desmoplasia [177, 184]. Whereas mutations in KRAS are sufficient for the initiation of PanINs, additional genetic hits in tumor suppressor genes such as TP53 (tumor protein p53), CDKN2A (cyclin-dependent kinase inhibitor 2A), and SMAD4 (mothers against decapentaplegic homologue 4) are required for PanINPDAC lineage [177, 184, 185]. A significant hallmark of PDAC is the presence of a dense stromal matrix, known as the tumor microenvironment, which accounts for up to $90 \%$ of the tumor bulk and has been shown to further promote PDAC progression [177, 179]. Unfortunately, targeting components of the tumor microenvironment using antistromal therapies to diminish PDAC progression has not been successful in clinical trials so far [179].

In CRC we previously demonstrated that mutp53 ${ }^{\mathrm{R} 248 \mathrm{Q} / \mathrm{W}}$ is stabilized by the HSP90 chaperone machinery, contributing to tumor progression and aggressiveness [56]. In PDAC, approximately $70 \%$ of patients carry mutations in TP53 which is mutated as a late genetic event during tumor development [177, 186, 187]. Thus, we aimed to investigate whether mutant p53 (mutp53) is also a cancer-relevant HSP90 client in PDAC. To understand the altered functions of p53 mutants in cancer cells, the next section describes the physiological functions of the tumor suppressor p53.

\subsubsection{Wildtype p53 - a tumor suppressor}

Since its first discovery in 1979, the tumor suppressor p53, referred to as the 'guardian of the genome', has been extensively studied [188-190]. The genetic structure of TP53 was shown to be highly evolutionarily conserved across species [191, 192]. It consists of an N-terminal transactivation domain, followed by a core DNA-binding domain and a C-terminal oligomerization domain [191, 193]. Under physiological conditions, p53 levels are tightly regulated and kept low, by its major antagonist, the E3 ubiquitin ligase MDM2, targeting it for proteasomal degradation [194-196]. However, a variety of different stressors such as nutrient starvation, DNA damage or hypoxia result in posttranslational modifications of both proteins, leading to an induction of p53 activity [193, 
195, 197]. Proper tetramerization of $\mathrm{p} 53$ is required for DNA binding, post-translational modifications as well as protein-protein interactions [197]. Following nuclear translocation, active p53 can bind to its target genes, and prevents the accumulation of mutagenic DNA [193, 195, 198]. As a transcription factor p53 regulates the expression of a large variety of genes involved in cell cycle progression (such as CDKN1A, GADD45A) as well as cellular survival and apoptosis (such as BAX, PUMA, NOXA) $[193,197,199]$. In a negative feedback loop, p53 induces the expression of MDM2, resulting in increased MDM2-mediated degradation of p53 to ensure normal cellular homeostasis [194, 195, 199].

Because of its pleiotropic functions in cellular protection and tumor suppression, mutation or ablation of TP53 is an essential step in human tumor development [188, 200, 201]. Indeed, TP53 has been shown to be mutated in approximately $50 \%$ of all human cancers, making it the most frequently mutated gene in a variety of cancer entities [202, 203].

\subsubsection{Mutant p53 - a tumor promoter}

The majority of TP53 mutations are missense mutations in the central region of the gene, the DNA binding domain (DBD) [202, 204-206]. Some of these mutations occur with high frequency among different cancer entities and are so called hotspot mutations [202, 205, 206]. These mutations in the DNA binding domain can be distinguished in two main groups: 'DNA-contact' mutants (e.g.: R273H, R248Q), directly affecting residues necessary for DNA binding or 'conformational' mutants harboring mutations which are causing structural changes (e.g.: R282W, R175H) [205, 207]. Most mutations in the DNA binding domain result in the loss of the DNA binding capacity, leading to a loss of wildtype (WT) p53 tumor suppressor function (loss-offunction, LOF) [202, 205]. Especially in early stages of tumor development, mutations of TP53 due to genotoxic stress occur only on one allele [208]. This results in an intermediate stage, characterized by the presence of heterocomplexes between mutp53 and the remaining WTp53 [205, 208]. This interaction of mutp53 and WTp53 results in diminished WTp53 activity, known as the dominant-negative effect of the mutant on the wildtype protein [202, 205, 208]. However, as tumors progress, loss-ofheterozygosity $(\mathrm{LOH})$ results in the loss of the remaining WTp53 allele, which is a prerequisite for stabilization of mutp53 in cancer cells [208, 209]. Most of the DBD 
mutants have been shown to be highly unfolded or less structurally stable than WTp53 [210-212]. The stabilization of these mutants is achieved by increased binding to the aberrantly activated HSP90 chaperone machinery (Figures 2 and 3), preventing it from proteasomal degradation (Figure 1), which results in elevated mutp53 levels in cancer cells [16, 56, 85, 213-215]. However, mutation of p53 can result in the exposure of a hydrophobic aggregation sequence within the DNA binding domain contributing to the formation of oligomers and prion-like proteins [216-219]. Co-aggregation of mutp53 and WTp53 in these prion-like structures is thought to contribute to the dominant negative effect $[219,220]$.

We propose that stabilization of mutp53 by HSP90 is a requirement for the gain of new tumorigenic functions (gain-of-function, GOF), providing a selection advantage for cancer cells [56, 208, 221, 222]. DBD hotspot mutants can no longer bind to the DNA to activate the transcriptional machinery, but still maintain the $\mathrm{N}$-terminal transactivation domain [210]. Therefore, several mechanisms have been described through which p53 mutants can still fulfil their transcriptional activity (Figure 6):

(I) Binding of mutp53 to other proteins and transcription factors can results in their enhanced functional or transcriptional activity (hyperactivation) [210, 223].

(II) Binding of mutp53 to other proteins or transcription factors (TF) can diminish their functional or transcriptional activity (inactivation) [223, 224].

(III) Interaction of mutp53 with other proteins and transcription factors may contribute to a change of existing signaling pathways (reprogramming) [210, 224]. 


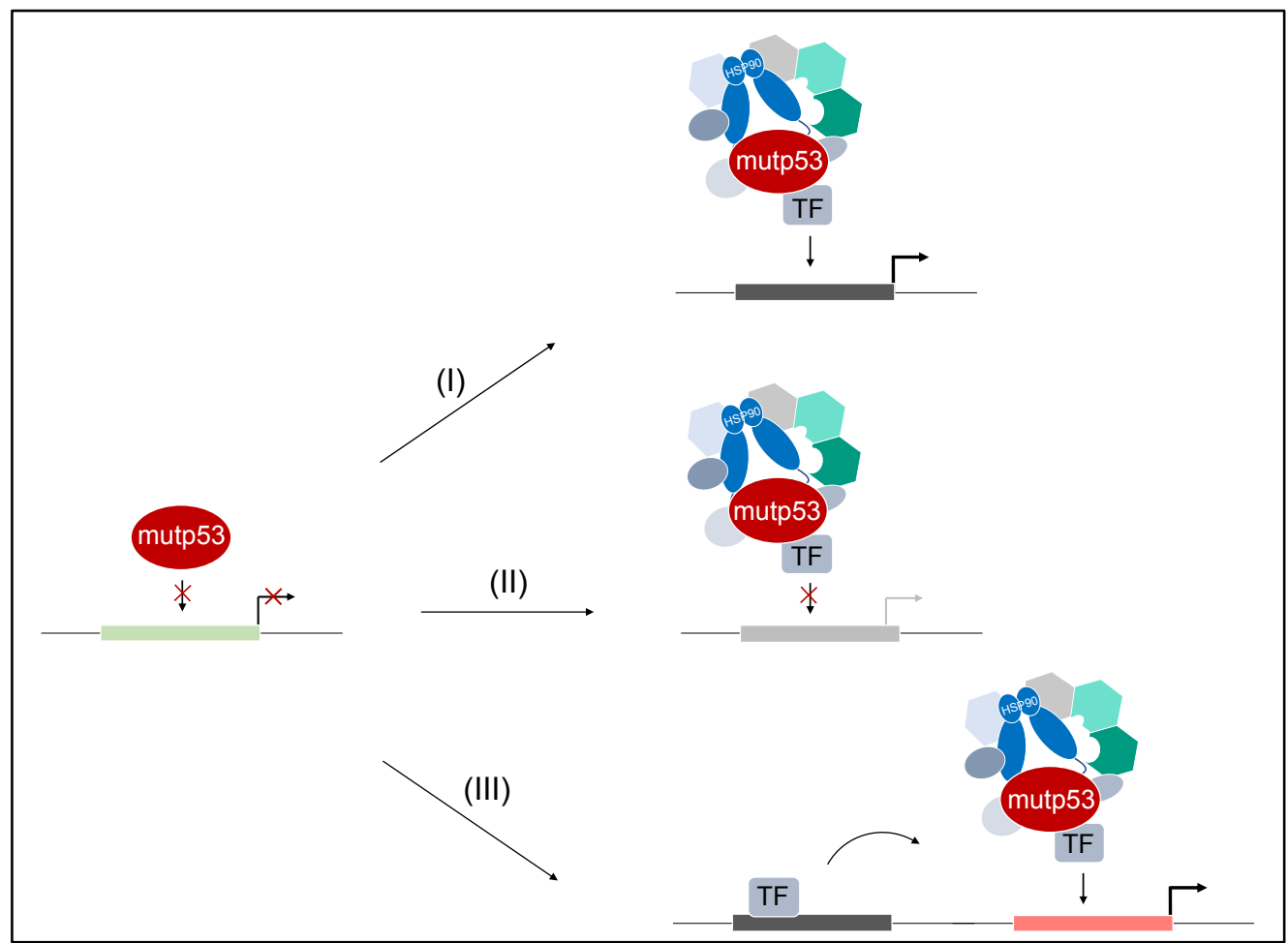

Figure 6: Gain-of-function of mutant p53 via interaction with other proteins. High stabilization of mutp53 via the HSP90 chaperone machinery is a prerequisite for gain-of-function (GOF). To fulfil its tumorigenic functions despite loss of DNA binding capacity, mutp53 interact with other proteins (such as co-factors) and transcription factors (TF). These interactions can trigger a hyperactivation (I), inactivation (II) or reprogramming (III) of these target proteins in order to drive tumor progression.

These mechanisms provide examples on how different p53 mutants (despite loss of DNA binding capacity) can gain new tumor promoting functions to support for example therapy resistance, invasion and metastasis of cancer cells [56, 210, 222, 224].

Because of the increasing network of mutp53 interactions with other proteins and regulation of pathways, several efforts have been made to target mutp53 in cancer therapy. In order to achieve this, two main strategies can be used:

(I) Small molecules to restore WTp53 conformation and activity to induce cell cycle arrest and apoptosis in cancer cells and to prevent oligomerization of mutp53 proteins [225-228].

(II) Destabilization of mutp53 proteins by inhibition of the HSP90 chaperone complex $[56,85,214,226,229]$. 
The underlying mechanism of the gain of function is highly dependent on the mutant itself as well as the cellular context [204, 210]. Due to the different structures and functions caused by distinct mutations on p53, each mutant has to be considered as an individual protein [204]. Different mutp53 variants might result in unequal gain of new functions and show diverse tumor promoting activities and prognostic potentials $[230,231]$. The existing controversies in the field of mutant p53 emphasize the necessity to further explore the exact mechanism of individual mutants and their relevance in tumor progression [231].

Thus, our research group investigates the specificity of the hotspot mutant p53 R2480/W, its stabilization via the HSP90 chaperone system and its GOF to support cancer progression.

\subsubsection{Specific gain of new tumorigenic functions by mutp53 $248 Q / W$}

Mutp53 $248 \mathrm{Q} / \mathrm{W}$ is a hotspot missense mutant in the DNA binding domain (contact mutant), that has been shown to be elevated in cancer cells and to correlate with a worse prognosis in cancer patients [56, 209, 230].

In a murine model for colorectal cancer, our group has previously shown that mutp53 ${ }^{\mathrm{R} 248 \mathrm{O} / \mathrm{W}}$ is highly stabilized by the HSP90 chaperone machinery [56]. It exerts its gain-of-function on migration through interaction with the transcription factor pSTAT3, resulting in hyperactivation of the transcription factor STAT3 via displacement of its phosphatase SHP2 [56, 210] (Figure 6). Hence, mutp53R248Q/W leads to an increased transcriptional regulation of STAT3 target genes involved in proliferation and invasion [56]. Concomitantly, an ablation of mutp53 decreases pSTAT3 levels, and diminishes tumor promoting target gene expression [56] (Figure 7). 


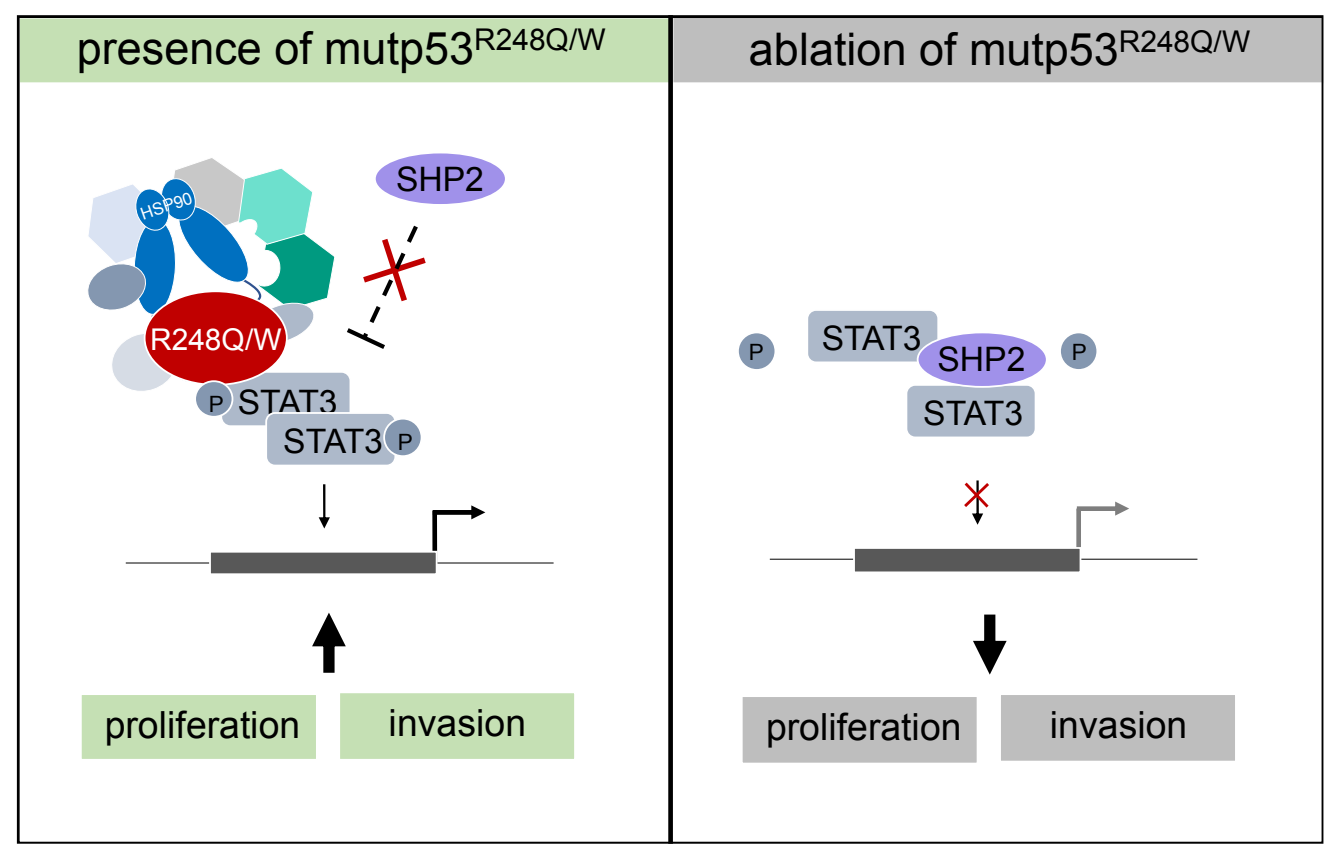

Figure 7: Gain-of-function of mutp53 ${ }^{\mathrm{R} 248 \mathrm{Q} / \mathrm{W}}$ on proliferation and invasion through interaction with pSTAT3 in CRC. In CRC mutp53 ${ }^{R 248 / w}$ is highly stabilized via the HSP90 chaperone machinery, which is a known prerequisite for its gain-of-function (GOF) effects. mutp53 $248 \mathrm{Q} / \mathrm{W}$ binds to phosphorylated STAT3 (pSTAT3), resulting in a hyperactivation of the transcription factor via displacement of the phosphatase SHP2. The increased PSTAT3 level support expression of genes involved in proliferation and invasion. Ablation of mutp53 ${ }^{\mathrm{R} 248 \mathrm{Q} / \mathrm{w}}$ strongly diminished pSTAT3 level and respective target gene expression.

Indeed, high levels of mutp53 $248 \mathrm{Q} / \mathrm{W}$ in colorectal cancer patients mostly correlated with high levels of pSTAT3 which was associated with poorer survival [56].

Importantly, the exact interaction partners of mutp53 ${ }^{\mathrm{R} 248 \mathrm{Q} / \mathrm{W}}$ and the functional outcome (Figure 6) highly depend on the molecular and cellular context [204]. Besides colorectal cancer, mutp53 ${ }^{\mathrm{R} 248 \mathrm{Q} / \mathrm{W}}$ has also been reported as potential tumor driver in breast cancer by upregulation of HER2 (human epidermal growth factor receptor 2) [232] or in ovarian cancer by inducing invasive gene expression via interaction with Rad21 [233]. In both cases, mutp53 ${ }^{\mathrm{R} 248 \mathrm{Q} / \mathrm{W}}$ has been suggested to interact with other proteins to promote tumor progression and aggressiveness [232, 233].

Taken together these data suggest the need to further investigate the specificity of different p53 mutants in cancer entities based on the molecular context. 


\section{$2.4 \quad$ PROJECT SCOPE}

The aim of this thesis is to investigate HSP90-stabilized proteins as therapeutic targets in cancer. We evaluated if the two HSP90 clients MIF and mutp53 are cancer-relevant HSP90 clients and thus, suitable drug targets in colorectal cancer and pancreatic ductal adenocarcinoma respectively.

\subsubsection{Macrophage migration inhibitory factor in colorectal cancer}

We aimed to investigate the role MIF in AOM/DSS induced colitis-associated tumor development (described in section 2.2.1 in more detail). This chemically induced cancer model is known to mimic the human patient situation better than previously described $A p c^{\text {min }}$ or xenograft mouse models [105, 149, 151]. To evaluate the impact of HSP90-stabilized MIF in CRC progression we made use of a conditional Mif knockout mouse as well as murine and patient-derived colonic tumor organoids. Our first and most important question was:

Are MIF levels elevated in AOM/DSS induced tumors and patient CRC samples?

To answer this question, mice were treated with AOM/DSS to induce tumor development. Mice were dissected 12 weeks after AOM injection and colon was prepared to evaluate the tumor burden and Mif level in CRC cells. By this approach, we were able to address the second most important question:

\section{Are colorectal tumors dependent on MIF?}

Since MIF as a pro-inflammatory cytokine is involved in immune and inflammatory responses, we first investigated the overall inflammation in established tumors (week 12 post-AOM) and during tumor initiation (3 days after DSS start: 'short' and 8 days after DSS stop: 'recovery'). Furthermore, we investigated tumorigenic mechanisms such as angiogenesis and proliferation in established tumors and recovering tissue.

To further clarify whether effects derive from epithelial cells or from stromal cells of the tumor bulk, Mif-depleted tumor organoids were prepared to assess tumorigenic MIF activity. 
Additionally, different HSP90 inhibitors (first-generation inhibitor 17AAG; secondgeneration inhibitors Ganetespib and Onalespib) were used to evaluate susceptibility of tumor organoids harboring elevated Mif levels, compared to normal colon and small intestinal epithelia-derived organoids.

\subsubsection{Mutp53 ${ }^{R 248 W}$ specificity in pancreatic ductal adenocarcinoma}

Using a panel of seven different cell lines, we wanted to elucidate the specificity of different mutp53 (mutant p53) variants in PDAC. Based on our results previously gained in a mouse model for colorectal cancer [56], we hypothesized that mutp53 ${ }^{\mathrm{R} 248 \mathrm{~W}}$ is stabilized through the HSP90 chaperone machinery resulting in a gain-of-function effect on proliferation and invasion via interaction with phosphorylated STAT3 (pSTAT3). Therefore, our most important question was:

Is mutant p53 stabilized via the HSP90 chaperone machinery in PDAC cells?

We evaluated the levels and stability of mutp53 in different PDAC cells. Using the two HSP90 inhibitors Gantespib and Onalespib, we investigated whether different mutp53 variants are stabilized by the HSP90 chaperone machinery. Using transwell migration and wound healing assays we elaborated on the functional role of different p53 mutants in migration. Next, we aimed to understand the underlying molecular mechanism, driving mutp53-dependent migration in PDAC.

Is mutp53-pSTAT3 complex formation necessary for migration in PDAC?

Therefore, co-immunoprecipitation experiments were performed to explore if different p53 mutants (and specifically R248W) are able to bind pSTAT3. To further analyze whether migration effects are due to a possible interaction with pSTAT3, migration assays were performed after STAT3 knockdown (siRNA) or STAT3 inhibition using the small molecule inhibitor Stattic. 


\section{RESULTS}

\subsection{PUBLICATION: MIF IN CRC}

\section{HSP90-STABILIZED MIF SUPPORTS TUMOR PROGRESSION VIA MACROPHAGE RECRUITMENT AND ANGIOGENESIS IN COLORECTAL CANCER}

Luisa Klemke ${ }^{1}$, Tiago De Oliveira ${ }^{2}$, Daria Witt ${ }^{1}$, Nadine Winkler ${ }^{1}$, Hanibal Bohnenberger ${ }^{3}$, Richard Bucala ${ }^{4}$, Lena-Christin Conradi ${ }^{2}$ and Ramona SchulzHeddergott ${ }^{1 *}$

${ }^{1}$ Institute of Molecular Oncology, University Medical Center Göttingen, Göttingen, Germany.

2 Department of General, Visceral, and Pediatric Surgery, University Medical Center Göttingen, Göttingen, Germany.

${ }^{3}$ Institute of Pathology, University Medical Center Göttingen, Göttingen, Germany.

${ }^{4}$ Departments of Medicine, Pathology, and Epidemiology \& Public Health, Yale School of Medicine and Yale Cancer Center, New Haven, CT, USA.

*Corresponding author: ramona.schulz@zentr.uni-goettingen.de

Cell Death and Disease (Feb 2021) 12:155

PMID: 33542244, DOI: 10.1038/s41419-021-03426-z

\section{Own contribution:}

Conducted experiments and data analysis for: Figures 2A-F, Figures 3B-D, Figures 4A-C, Figures 5A-C, E, G and H-J, Figures 6D-I, Figures 7A-D, Figures S1A-C, Figures S2A-C and G-I, Figures S3A-C and G-K, Figures S4A, B, Figures S5A-C. Data analysis for: Figures 1C, E, Figures 4E-G, Figures 5D, F, Figure 7E, Figure S2F, as well as preparation of additional supplemental material. Involved in the conceptualization, methodology, acquisition, analysis and interpretation of data, figure arrangement, writing and revising the manuscript. 


\title{
Hsp90-stabilized MIF supports tumor progression via macrophage recruitment and angiogenesis in colorectal cancer
}

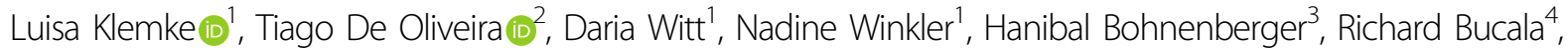 \\ Lena-Christin Conradi ${ }^{2}$ and Ramona Schulz-Heddergott (1) ${ }^{1}$
}

\begin{abstract}
Macrophage migration inhibitory factor (MIF) is an upstream regulator of innate immunity, but its expression is increased in some cancers via stabilization with HSP90-associated chaperones. Here, we show that MIF stabilization is tumor-specific in an acute colitis-associated colorectal cancer (CRC) mouse model, leading to tumor-specific functions and selective therapeutic vulnerabilities. Therefore, we demonstrate that a Mif deletion reduced CRC tumor growth. Further, we define a dual role for MIF in CRC tumor progression. Mif deletion protects mice from inflammationassociated tumor initiation, confirming the action of MIF on host inflammatory pathways; however, macrophage recruitment, neoangiogenesis, and proliferative responses are reduced in Mif-deficient tumors once the tumors are established. Thus, during neoplastic transformation, the function of MIF switches from a proinflammatory cytokine to an angiogenesis promoting factor within our experimental model. Mechanistically, Mif-containing tumor cells regulate angiogenic gene expression via a MIF/CD74/MAPK axis in vitro. Clinical correlation studies of CRC patients show the shortest overall survival for patients with high MIF levels in combination with CD74 expression. Pharmacological inhibition of HSP90 to reduce MIF levels decreased tumor growth in vivo, and selectively reduced the growth of organoids derived from murine and human tumors without affecting organoids derived from healthy epithelial cells. Therefore, novel, clinically relevant Hsp90 inhibitors provide therapeutic selectivity by interfering with tumorigenic MIF in tumor epithelial cells but not in normal cells. Furthermore, Mif-depleted colonic tumor organoids showed growth defects compared to wild-type organoids and were less susceptible toward HSP90 inhibitor treatment. Our data support that tumor-specific stabilization of MIF promotes CRC progression and allows MIF to become a potential and selective therapeutic target in CRC.
\end{abstract}

\section{Introduction}

Macrophage migration inhibitory factor (MIF), which was originally discovered as a secreted proinflammatory cytokine with a central role in immune and inflammatory responses, has also been identified as a tumor promoter ${ }^{1,2}$.

\footnotetext{
Correspondence: Ramona Schulz-Heddergott

(ramona.schulz@zentr.uni-goettingen.de)

${ }^{1}$ Institute of Molecular Oncology, University Medical Center Göttingen, Göttingen, Germany

${ }^{2}$ Department of General, Visceral, and Pediatric Surgery, University Medical Center Göttingen, Göttingen, Germany

Full list of author information is available at the end of the article

Edited by M. Agostini
}

\section{(c) The Author(s) 2021}

MIF is known to exert effects in epithelial cancer cells, stromal fibroblasts, endothelial cells, and immune cells ${ }^{3-10}$. In tumors, the major source of MIF is the epithelial cells themselves ${ }^{11-13}$, followed by a minor secretory contribution from constituents of the tumor microenvironment, such as stromal and inflammatory cells ${ }^{5,14,15}$. Therefore, tumor cells aberrantly elevate MIF expression via Hsp90mediated protein stabilization ${ }^{10,11,16}$. The HSP90 chaperone machinery is a prerequisite for tumorigenesis because it stabilizes oncogenic and tumor-promoting proteins, protecting them from degradation ${ }^{17,18}$. We previously

\footnotetext{
(c) Open Access This article is licensed under a Creative Commons Attribution 4.0 International License, which permits use, sharing, adaptation, distribution and reproduction changes were made. The images or other third party material in this article are included in the article's Creative Commons license, unless indicated otherwise in a credit line to the material. If material is not included in the article's Creative Commons license and your intended use is not permitted by statutory regulation or exceeds the permitted use, you will need to obtain
} permission directly from the copyright holder. To view a copy of this license, visit http://creativecommons.org/licenses/by/4.0/. 
identified MIF as an Hsp90-stabilized protein in breast cancer cells ${ }^{11}$.

Colorectal cancer (CRC) patients also present elevated MIF levels, which are associated with a worse prognosis $^{12,15,19-22}$. Among cancers, CRC has the third highest incidence ${ }^{23}$. Previous in vitro studies in human CRC cells showed that MIF increases proliferation, angiogenesis, and migration ${ }^{12,24,25}$. Functionally, MIF can bind to its main receptor CD74 to activate p38, MAPKs, or PI3K/ $\mathrm{AKT}$, which induces the expression of angiogenic factors ${ }^{4,12,24,26-28}$. Furthermore, MIF regulates therapeutic resistance via regulation of STAT3, MAPKs, AMPK, or hypoxia-dependent mechanisms ${ }^{28-31}$. Other studies using CT26 allograft models support that MIF promotes CRC progression $^{12,24}$. In vivo, it has been shown that MIF stimulates the early stages of small intestinal adenomas in $A p c^{\text {min }}$ mice $^{27}$. Although all these studies showed a positive correlation between aberrant MIF function and CRC growth, an in vivo model of causative and severe CRC that mimics the human CRC was not available.

In our study, we investigated whether MIF promotes tumor growth in an autochthonous colorectal azoxymethane (AOM)/dextran sodium sulfate (DSS) mouse model and whether MIF can serve as a potential drug target. Because of the tumor-specific Hsp90-mediated stabilization of MIF, this protein could be selectively targeted in CRC. Our data suggest that MIF increases CRC growth and supports tumor-specific macrophage recruitment, tumor cell proliferation, and neoangiogenesis without affecting overall inflammation in established tumors.

Strikingly, a recent study in a mouse model of chronic colitis-dependent CRC reported a tumor-protective role for $\mathrm{MIF}^{32}$. This phenomenon was not observed in neither the $A p c^{\text {min }}$ mouse model ${ }^{27}$ nor several other in vivo cancer studies, including myc-induced lymphoma, chronic lymphocytic leukemia, breast, prostate, bladder, and skin cancer ${ }^{3,4,11,33-38}$. An important difference between the previous work and our study is that we used a mouse model of acute colitis-associated CRC, which is more similar to human sporadic $\mathrm{CRC}^{39}$. Importantly, in our sporadic CRC model, MIF as a tumor-promoting factor is selectively targetable in tumor cells by inhibiting Hsp90, supporting a strong rationale for MIF as a potential therapeutic target in sporadic CRC.

\section{Results}

MIF supports tumor growth in a mouse model of CRC

Given the importance of MIF in cancer and to determine whether MIF supports CRC tumorigenesis, we used the severe CRC AOM/DSS mouse model, which includes one phase of acute colitis (Fig. 1A). After a recovery phase, mice exclusively develop tumors within 12 weeks in the large intestine ${ }^{40}$. At 5 weeks post-AOM, when the tumors were macroscopically visualized by colonoscopy, $\mathrm{Mif}^{-/-}$
A

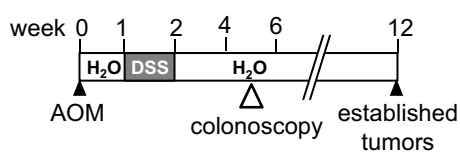

B 5 wks post-AOM

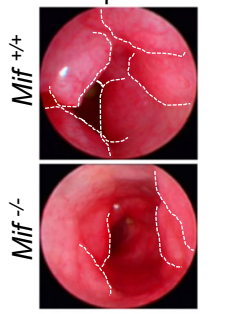

D

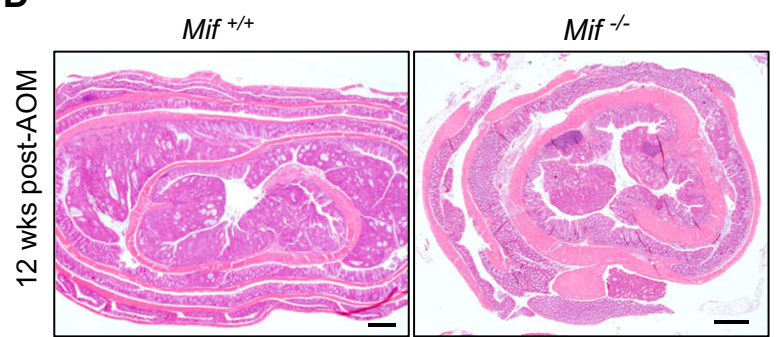

C 5 wks post-AOM
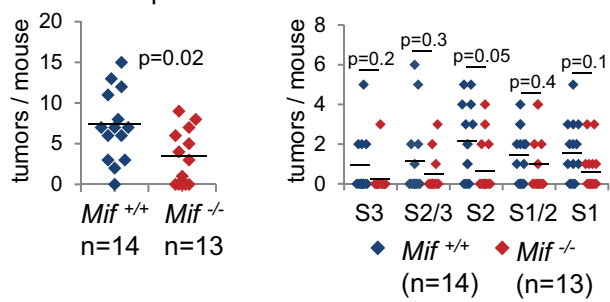

E

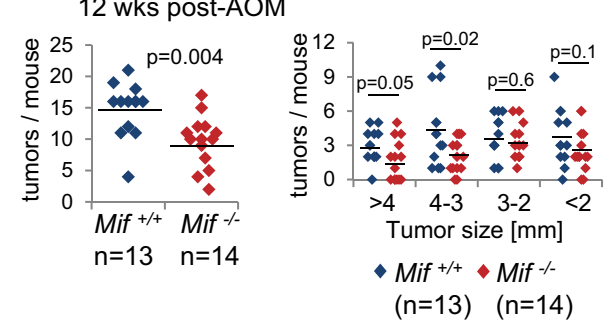

Fig. 1 MIF supports tumor growth in a CRC mouse model. A Schematic of the AOM/DSS CRC mouse model. Visualization of tumor burden started at week 5 by colonoscopy. At 12 weeks post-AOM injection, established tumors were analyzed. B Representative colonoscopy images of the colonic lumen of the indicated genotypes at week 5 post-AOM. Dashed lines indicate the tumor borders. $\mathbf{C}$ Average number of tumors (left) and tumor sizes (right) in $\mathrm{Mif}^{+/+}$and $\mathrm{Mif}^{-/-}$mice at 5 weeks post-AOM injection. Numbers were determined by colonoscopy in living mice. S1 = small tumors to S3 = larger tumors. D Representative H\&E-stained colonic tissues with tumors from Mif ${ }^{+/+}$and Mif ${ }^{-/-}$mice at week 12 post-AOM injection. Scale bars, $600 \mu \mathrm{m}$. E Average number of tumors per mouse (left) and tumor sizes (right) of the indicated mice at 12 weeks post-AOM injection. $\mathbf{C}$, E $n$, mouse numbers. Black lines, mean. Student's $t$ test of indicated groups. 
A

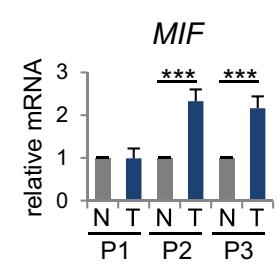

B

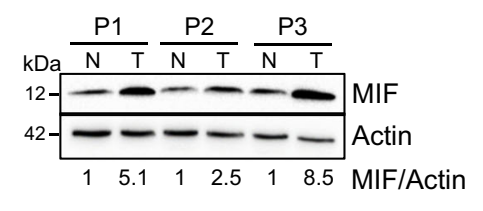

C

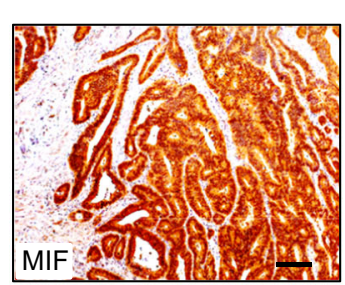

D

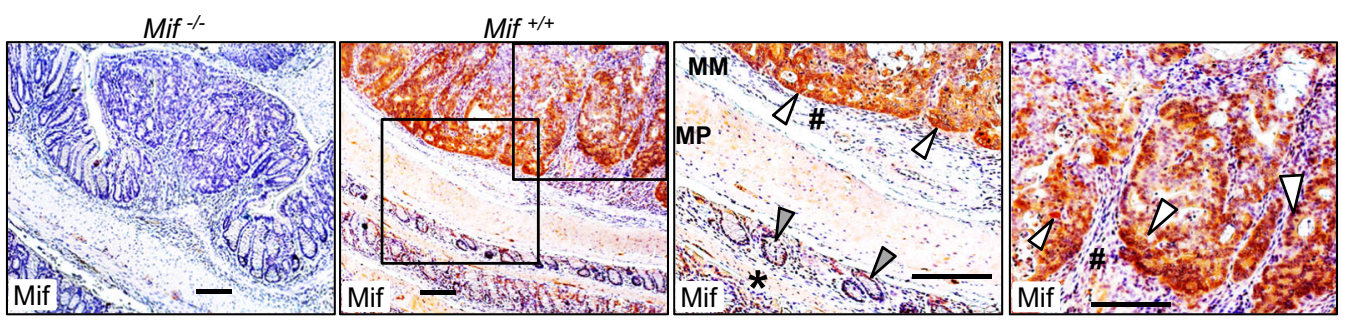

E

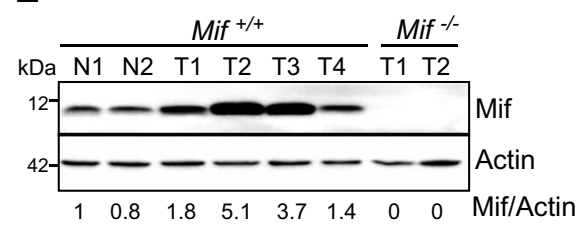

$\mathbf{F}$

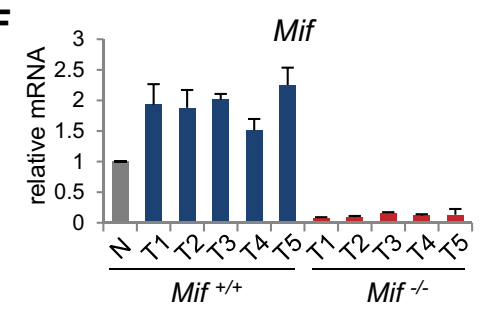

Fig. 2 MIF levels are elevated in CRC cells. A Relative MIF mRNA expression of individual human CRC patients (P1-P3). Matched pairs of adjacent epithelia ('N') and their tumors ('T') were evaluated by qRT-PCR, and RNA levels were normalized to those of RPLPO mRNA. Mean \pm SD of four technical replicates. Student's $t$ test was performed for comparison of indicated groups; ${ }^{* * *} p \leq 0.001$. B MIF protein levels in matched pairs of tumor ('T') and adjacent epithelial ('N') tissues from three individual human CRC patients (P1-P3). Immunoblot analysis. Actin, loading control. MIF expression ratios (MIF/Actin) were calculated by densitometry, normalized to the loading control, relative to the respective epithelium (' $\mathrm{N}$ '). $\mathbf{C}$ Histological MIF staining in a colonic tumor from a human CRC patient. Scale bar, $200 \mu \mathrm{m}$. D Representative histological Mif staining in colonic tumors from Mif ${ }^{+/+}$and Mif $^{-/-}$ mice. Mif ${ }^{\prime-}$ tumors served as negative control. Rectangles represent the area at a higher magnification. MM, muscularis mucosae; MP, muscularis propria. White arrows, tumor epithelial cells with elevated MIF levels. \#, stromal cells of mucosae. Gray arrows, epithelial cells within the next surrounding colonic tissue layer. ${ }^{*}$, MM and MP of the next surrounding layer. Note that normal epithelial cells from untransformed regions of the colon show low MIF levels (gray arrow) compared to those in tumor cells (white arrow). Scale bars, $200 \mu m$. E Mif protein levels in murine single tumor samples ( $\left.\mathrm{T}^{\prime}\right)$ compared to matched normal epithelium ('N') from Mif ${ }^{+/+}$mice. Mif ${ }^{-1-}$ tumors served as a negative staining control. Mif ratios were calculated as described in (B). F Mif mRNA level in single tumor samples (' $\left.\mathrm{T}^{\prime}\right)$ of Mif $^{+/+}$and Mif ${ }^{-/-}$mice compared to pooled normal epithelium $\left({ }^{\prime}{ }^{\prime}\right)$ of Mift $^{+/+}$mice $(n=2)$. mRNA levels were normalized to those of Rplpo mRNA. Means \pm SD of four technical replicates.

mice showed a reduction in the tumor burden (Fig. 1B). Quantification of colonic tumors by a scoring system ${ }^{41,42}$ revealed a reduction in tumor multiplicity in $\mathrm{Mif}^{-1-}$ mice (Fig. 1C). Moreover, at 12 weeks post-AOM, during which the CRC tumors are well established, MIF deficiency decreased tumor burden and numbers (Fig. 1D, E). In summary, MIF supports tumor growth in an acute colitisassociated CRC mouse model.

\section{MIF levels are elevated in CRC cells}

During tumorigenesis, MIF protein levels are increased $^{12,27}$. Our data confirm elevated MIF levels in cancer cells from CRC patients (Figs. 2A-C, S1A). Compared to the moderate increase in MIF mRNA levels (Figs. 2A, S1A), MIF protein levels were strongly increased in tumors from patients (Fig. 2B, C). Similar to the patient tumors, established AOM/DSS-induced tumors confirmed tumor-specific elevation of MIF expression (Fig. 2D). Intriguingly, epithelial cancer cells express high levels of MIF compared to those in the normal surrounding epithelium (Fig. 2D), indicating that the major source of MIF is tumor epithelial cells. Measurement of MIF expression in murine tumor lysates indicated increased MIF expression in tumors compared to normal colonic tissue (Figs. 2E, F and S1B, C). Taken together, these results confirm an enhanced tumorspecific increase in MIF occurrence within the epithelial tumor compartment.

\section{A Mif deletion protects mice from inflammation-associated cancer initiation}

As a proinflammatory cytokine, MIF regulates immune responses and is suggested to be a link between inflammation and cancer ${ }^{1,2}$. Therefore, we hypothesized that the 
loss of Mif expression protects mice during the colitisassociated phase of tumor initiation. Indeed, during the recovery phase, colonic tissue damage and epithelial cell loss, as reflected by the inflammatory score, were increased in $\mathrm{Mif}^{+/+}$mice compared to $\mathrm{Mif}^{-1-}$ mice and were accompanied by increased immune cell infiltration (Fig. 3A, B). To further examine the inflammatory response, we histologically analyzed the immune cell composition within the tumor microenvironment. Infiltrates from the colonic tissue of $\mathrm{Mif}^{+/+}$mice had higher percentages of CD3-positive (T-lymphocyte marker), MPO-positive (neutrophil/granulocyte marker), and FoxP3-positive (regulatory T-cell marker) cells than did colonic tissue from $\mathrm{Mif}^{-/}$mice (Figs. 3C, S2A). Immune infiltrates and the inflammatory score showed a positive correlation (Figure S2B). Interestingly, CD68-positive (monocyte/macrophage marker) cell infiltration was unchanged between the two mice groups (Fig. 3C, S2A). Similar to the changes in the inflammatory cell composition, the expression of inflammation-associated cytokines was downregulated in $\mathrm{Mif}^{-1-}$ tissues during the recovery period, confirming a reduction in inflammation in the absence of Mif (Fig. 3D, S2C). Consistent with the protective effect of Mif deletion during recovery, $\mathrm{Mif}^{-/}$ mice showed a reduced overall inflammatory response under DSS administration (Figures S2D-G). Furthermore, since MIF inhibits p53 activity ${ }^{11,43}$, we pursued whether MIF interferes with the DNA damage response and apoptosis in response to AOM treatment. Surprisingly, neither the levels of phosphorylated histone H2A.X
A

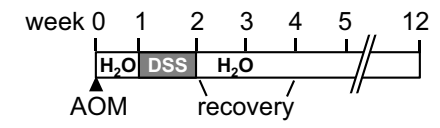

C
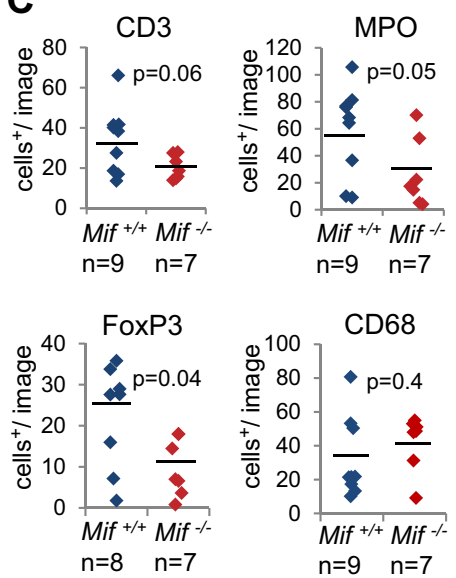

B
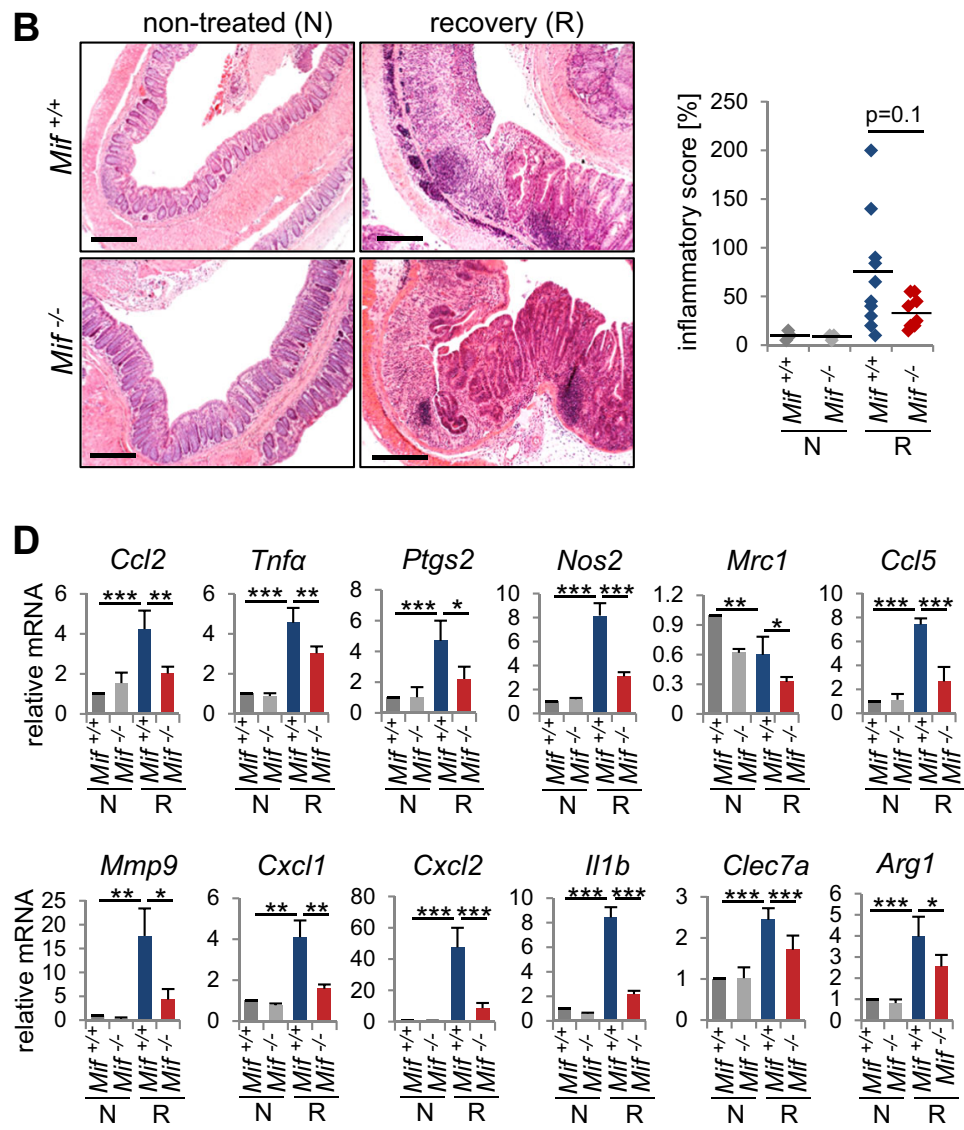

Fig. 3 A Mif deletion protects mice from inflammation-associated cancer initiation. A Schematic of the AOM/DSS mouse model. CRC was initiated by a single AOM injection, followed by one cycle of DSS administration. At 8 days of post-DSS treatment, mice were analyzed for recovery. B Representative H\&E staining of colonic tissues from the indicated mice 8 days post-DSS (recovery = ' $R$ ') or from untreated control mice (' $N$ '). Scale bars, $300 \mu \mathrm{m}$. (Right) The 'inflammatory score' was assessed based on tissue morphology and immune cell infiltration. (Left) Nontreated mice, $n=3 /$ group; recovery Mif ${ }^{+/+}$mice, $n=10$, Mif $^{-1-}$ mice, $n=8$. Black lines, mean. $p$ values were calculated with ANOVA Bonferroni's multiple comparison test; $p=0.0455$. Indicated groups, calculated with Student's $t$ test. C Quantification of the indicated histological staining of colonic tissues 8 days post-DSS ('recovery') in Mif ${ }^{+/+}$and Mif ${ }^{-1-}$ mice. $n$, mouse numbers. Five to seven images (area $=\times 40$ magnification) per mouse were analyzed for positively stained stromal cells. Black lines, mean. D mRNA levels of inflammatory genes in recovering ('R') and nontreated (' $N$ ') colonic tissues. Single samples from the indicated genotypes/groups were pooled (nontreated, $n \geq 3$; recovering tissue, $n \geq 4$ ). qRT-PCR was performed, and the expression levels were normalized to those of Rp/p0 mRNA. Means \pm SD of $\geq 3$ technical replicates/pools, each pipetted. C, D $p$ values were calculated with Student's $t$ test comparing the indicated groups. ${ }^{*} p \leq 0.05,{ }^{* *} p \leq 0.01,{ }^{* *} p \leq 0.001$. 
(a DNA damage marker) nor the expression of p53 target genes (Mdm2, Cdkn1a, Ccnd1, Gadd45a, and Bax) was altered in colonic tissues in $\mathrm{Mif}^{-1-}$ mice compared to those in $\mathrm{Mif}^{+/+}$mice, suggesting that MIF failed to regulate an AOM-induced p53-dependent response in colonic epithelia (Figures S2H, I).

Overall, a Mif deficiency protected mice during the early phases of inflammation in the AOM/DSS model and demonstrated that during colitis-associated tumor initiation, MIF acts as a proinflammatory cytokine.

\section{MIF supports CRC development via tumor-specific macrophage recruitment and angiogenesis without affecting overall inflammation}

To determine whether MIF also acts as an inflammatory cytokine to support established tumors, we analyzed the expression of inflammatory markers at 12 weeks post-AOM. Interestingly, immunohistological staining (Figure S3A) and their corresponding quantifications (Fig. 4A) did not show any differences in the extent of infiltrating lymphocytes, regulatory $\mathrm{T}$-cells or neutrophils/granulocytes within established tumors. In line with these findings, an assessment of inflammatory cytokines from tumor lysates failed to show major differences between Mif-expressing and Mif-deficient tumors, although all cytokines were upregulated in tumor samples ('T') compared to normal epithelium samples from untreated animals ('N') (Figure S3B). However, interestingly, CD68-positive macrophage/ monocyte infiltration was decreased in $\mathrm{Mif}^{-1-}$ tumors compared to $\mathrm{Mif}^{+/+}$tumors (Fig. 4B, upper panel), supporting the function of MIF as a chemokine to mediate macrophage recruitment ${ }^{3,4,44}$. To clarify whether elevated MIF expression in tumor cells mediates tumor-specific macrophage recruitment, we monitored the adjacent epithelium (Fig. 4B, lower panel). Indeed, macrophages specifically infiltrated tumors, suggesting that MIF regulates the chemotaxis of tumor-associated macrophages to promote CRC tumorigenesis. Tumorassociated macrophages are known to secrete tumorpromoting cytokines during cancer progression to stimulate tumor cell proliferation and angiogenesis ${ }^{45,46}$. Indeed, levels of Vegfa, an angiogenic cytokine known to be secreted by macrophages ${ }^{46,47}$, are reduced in Mif $^{-/-}$ tumors (Figs. 4C, F, G, S3C). Moreover, in established CRC tumors, $\mathrm{Mif}^{+/+}$mice showed stronger vessel formation, as indicated by CD31-positive staining, compared to $\mathrm{Mif}^{-1-}$ mice (Fig. 4D, E). Immunoblots confirmed increased activation of proangiogenic factors such as p38 and ERK in Mif-containing samples (Fig. 4F), an effect described previously ${ }^{3,10,12,27,48}$. MIF also affected tumor cell proliferation in AOM/DSS-induced tumors (Fig. 4H), which might explain the smaller tumors observed in $\mathrm{Mif}^{-/-}$mice (Fig. 1D, E).
Interestingly, Akt activity remained unchanged in Mifdeficient AOM/DSS tumors (Figure S3D), despite strong evidences that MIF activates PI3K/AKT in $\mathrm{CRC}^{26}$ and other cancers ${ }^{49,50}$.

Given that MIF also intrinsically regulates apoptosis via p53, e.g., in HER2-positive breast cancer or macrophages $^{11,51}$, we clarified whether the loss of MIF expression also activates p53 target genes in AOM/DSS tumors. In our CRC mouse model, Mif deficiency did not upregulate the expression of p53 target genes involved in apoptosis (e.g., Bax, Bcl2l1, Bcl2, and Mcl1) (Figure S3E). TUNEL staining confirmed the lack of altered apoptosis in $\mathrm{Mif}^{-/-}$tumors (Figure S3F). However, the expression of the cell cycle inhibitor $p 21 / C d k n 1 a$ was upregulated in Mif $^{-/}$tumors (Figure S3E), supporting the diminished proliferation upon MIF loss (Fig. 4H).

To assess whether angiogenesis and proliferation are affected during the recovery phase, we evaluated Vegfa, CD31, and Ki67 expression in colonic tissues at 8 days postDSS (Figures S3G-K) and found that neither vessel formation and Vegfa expression nor proliferation was dependent on the presence of MIF during colonic tissue recovery.

Albeit our data confirmed that MIF supports inflammatory processes during colitis-associated tumor-initiating phases, we identified that in established tumors, MIF contributes to tumor-specific macrophage recruitment, tumor cell proliferation, and vessel formation without affecting overall inflammatory responses. Whether these infiltrated macrophages release proangiogenic cytokines ${ }^{45,47}$ or whether MIF regulates angiogenic pathways in tumor cells themselves ${ }^{52}$ must be further elucidated.

\section{The CD74-MIF receptor complex facilitates the expression of proangiogenic factors in human CRC cells}

MIF functions through CD74/CD44 and/or CXCR2/4 receptor complexes in proliferation, angiogenesis, and with its chemokine-like properties in monocyte and leukocyte recruitment ${ }^{3,8,53-55}$. The CD74 receptor is the main MIF receptor ${ }^{53,56}$. Since the expression of Vegfa is downregulated in Mif-deficient tumors (Figs. 4C, S3C), we examined whether tumor cells themselves are able to express angiogenic genes via MIF binding to CD74 to activate MAP kinases to induce VEGF and IL8 expression $^{12,24,26-28}$. First, we used the CD74-expressing (Fig. 5A) and MIF secreting HCT116 cell line ${ }^{29,57}$. Indeed, knockdown of either MIF or CD74 in HCT116 cells reduced VEGFA and CXCL8/IL8 expression supporting a MIF-CD74 axis (Figs. S4A, 5B). Second, we used DLD-1 cells which do not express CD74 and are not shown to secrete MIF (Fig. 5A), thus, missing the prerequisites (secreted MIF and CD74) for a MIF-CD74 axis. As expected, in parental DLD-1 cells, depletion of MIF did not show any alterations in VEGFA and CXCL8/IL8 expression (Figs. S4B, 5C). Moreover, supplementation of 
A

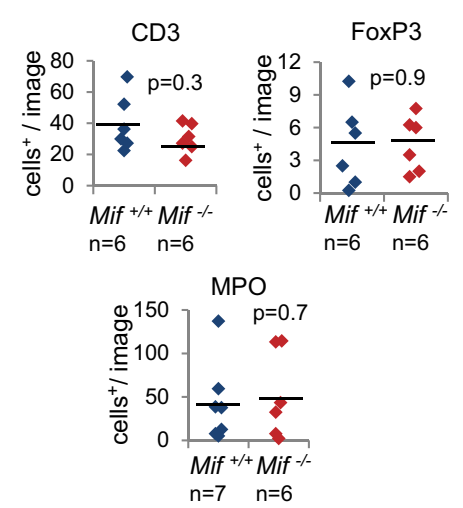

C

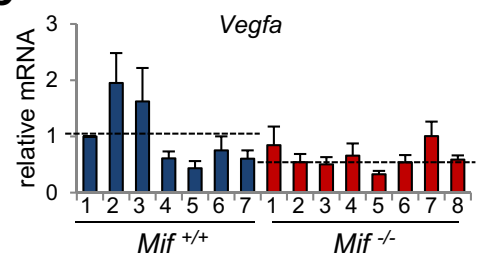

$\mathbf{F}$

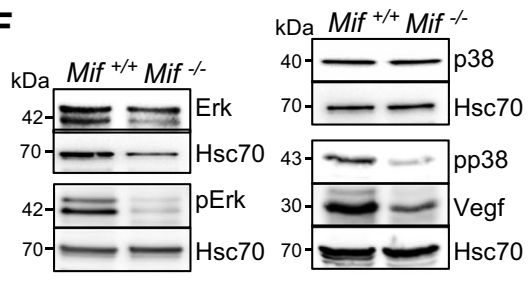

B
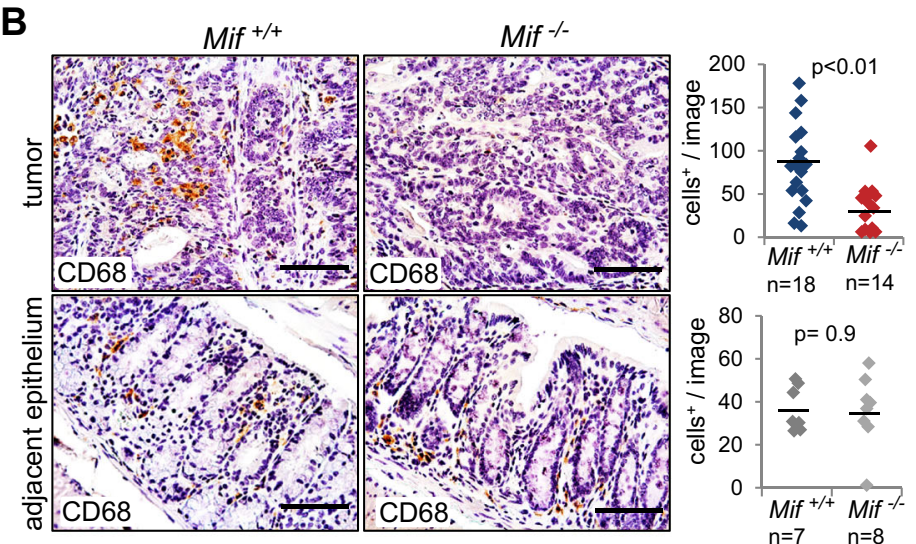

D
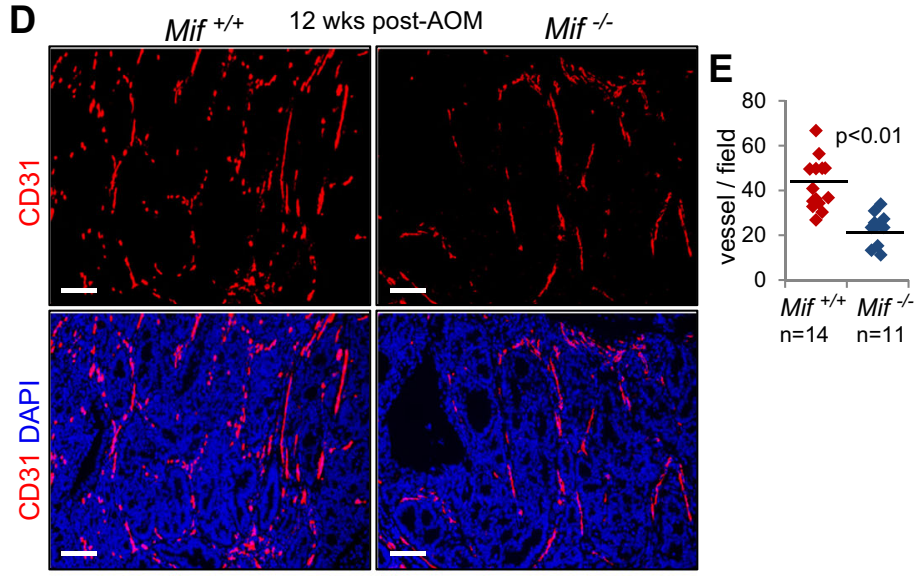

G

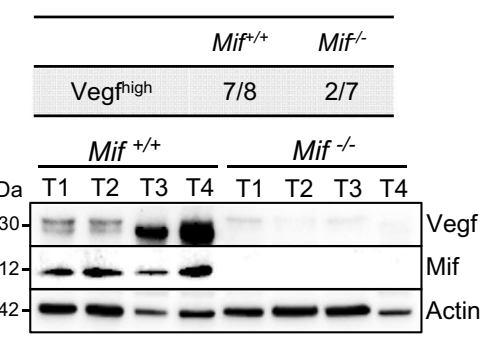

H

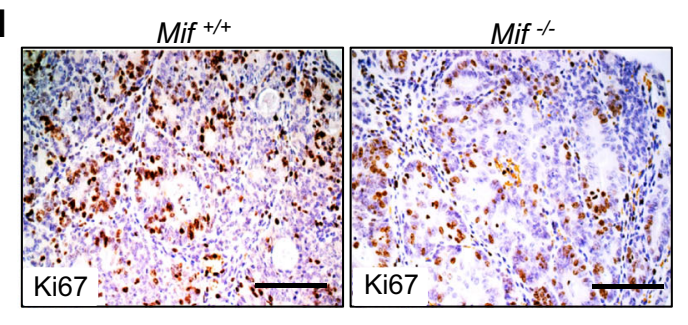

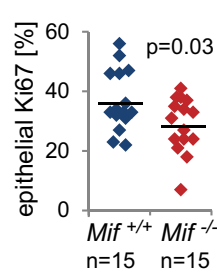

Fig. 4 MIF accelerates proliferation and angiogenesis in established colorectal tumors. A Quantification of the indicated histological staining of $\mathrm{Mif}^{+/+}$and $\mathrm{Mif}^{-/-}$tumors at 12 weeks post-AOM treatment. At least 2 images (area $=\times 40$ magnification) per tumor were viewed for positive stromal cells. $n$, tumor number from 3 mice each. B Representative CD68 staining of indicted tumors and their corresponding adjacent normal epithelium at 12 weeks post-AOM injection. Scale bars, $100 \mu \mathrm{m}$. (Right) For each tumor, $\geq 3$ images were taken, and positive staining was quantified. There were 18 tumors from $7 \mathrm{Mif}^{+^{-/}}$mice and 14 tumors from $8 \mathrm{Mif}^{-1-}$ mice. For adjacent epithelium, $\geq 5$ images (area $=\times 40$ magnification) per mouse were viewed and counted for positive stromal cells. $n$, mouse numbers. C mRNA levels of Vegfa in single tumors of different mice $\left(\right.$ Mif $\left.^{+/+}, n=7 ; \mathrm{Mif}^{-1-} n=8\right)$, relative to housekeeping gene, Hprt7. Means \pm SD of four technical replicates. Black dashed line, mean. D Representative immunofluorescence for CD31 in tumors from Mif ${ }^{+/+}$and Mif ${ }^{\prime-}$ mice at 12 weeks post-AOM. DAPI, counterstaining. Scale bars, $100 \mu \mathrm{m}$. E Quantification of the vessel number from (D). Vessels from at least five images (area $=\times 40$ magnification) per tumor were counted. A 'vessel' is defined as one separate fragment of CD31 staining. $n=$ number of tumors out of 5 mice each. $\mathbf{F}$ Immunoblot analysis of $\mathrm{Mif}^{+/+}$and $\mathrm{Mif}^{-{ }^{-}-}$tumors at 12 weeks post-AOM with pooled samples ( $n \geq 6$ tumors per condition). Hsc70, loading control. G Summary of $\mathrm{Mif}^{+/+}(n=8)$ and $\mathrm{Mif}^{-{ }^{\prime}-}(n=7)$ tumors analyzed by immunoblot. (Bottom) Representative immunoblot analysis of single $\mathrm{Mif}^{+/+}$and $\mathrm{Mif}^{-1-}$ tumors at 12 weeks post-AOM. Compared to a reference tumor $\left(\mathrm{T} 1\right.$ of $\left.\mathrm{Mif}^{+/+}\right)$, Vegf figh' means higher or same protein levels than the reference tumor. Actin, loading control. $\mathbf{H}$ Representative histological staining for Ki67 in tumors from $\mathrm{Mif}^{+/+}$and $\mathrm{Mif}^{-/-}$mice and quantification at 12 weeks post-AOM. Scale bars, $100 \mu \mathrm{m}$. Quantification of Ki67 staining counted within 2-4 images (area $=\times 40$ magnification) per tumor. $n$, tumor number; both groups showed 15 tumors from 5 mice. A, B, E, H Black lines, mean. $p$ values were calculated by Student's $t$ test. 
A

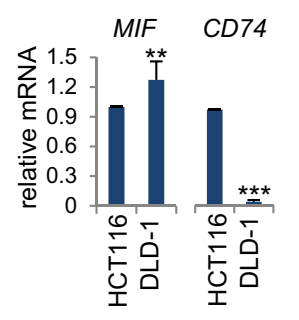

B

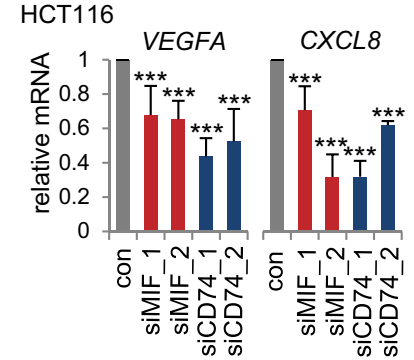

C DLD-1

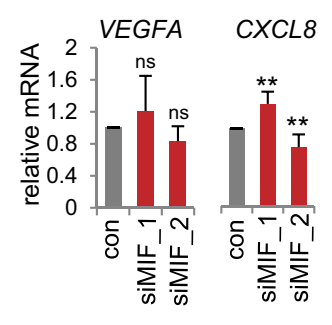

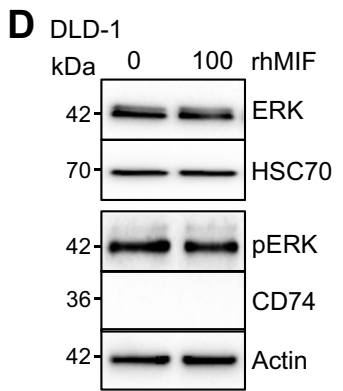

E

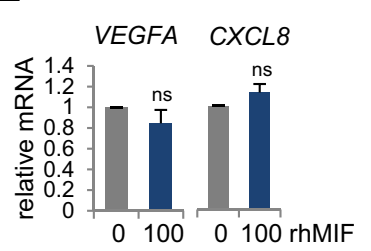

$F$

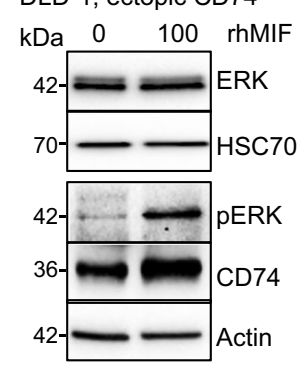

G ectopic $\mathrm{CD} 74$

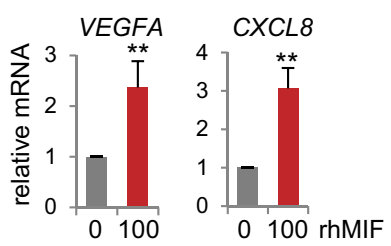

H

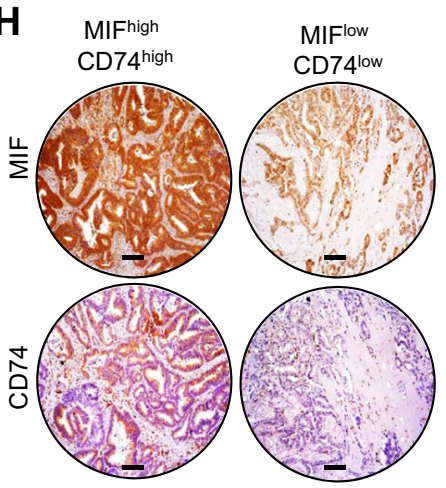

I

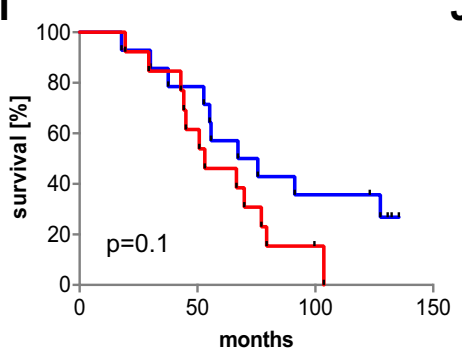

- MIF high $C D 74^{\text {low }}, \mathrm{n}=14,71.4 \mathrm{mo}$ - MIF ${ }^{\text {high }} \mathrm{CD} 74^{\text {high }}, \mathrm{n}=13,53.1 \mathrm{mo}$
J

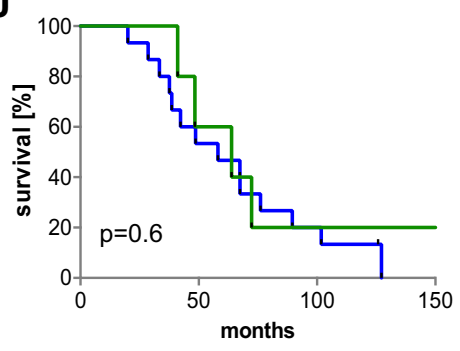

- MIFlow $C D 74^{\text {low }}, \mathrm{n}=15,58.1 \mathrm{mo}$

- MIF ${ }^{\text {low }} C D 74^{\text {high }}, \mathrm{n}=5,63.8$ mo

Fig. 5 The CD74-MIF receptor interaction facilitates the expression of angiogenic factors in CRC cells. A qRT-PCR analysis of the relative mRNA levels of MIF and CD74 in HCT116 and DLD-1 CRC cells. Normalized to those of RPLPO. Means \pm SD of 2 biological replicates with 3 technical replicates each. B, C Expression analysis of the indicated angiogenic genes in HCT116 (B) or DLD-1 (C) cells after siRNA-mediated knockdown of MIF or CD74 for $72 \mathrm{~h}$; a scrambled siRNA served as the control ('con'). qRT-PCR were normalized to HPRT1 or RPLPO, respectively. Means \pm SD of $\geq 3$ technical replicates from two biological replicates. D, F Angiogenic pathway analysis in CD74-deficient DLD-1 cells. $48 \mathrm{~h}$ after DLD-1 cells were transfected with an empty control (D) or CD74 overexpression plasmid (F), they were treated with $100 \mathrm{ng} / \mathrm{mL}$ recombinant human MIF (rhMIF) for $24 \mathrm{~h}$. Immunoblots of CD74, pERK, and total ERK. Actin and Hsc70, loading controls. E, G Angiogenic marker expression (VEGFA, CXCL8). DLD-1 cells were treated as described in $(\mathbf{D}, \mathbf{F})$ followed by qRT-PCR. Expression of the indicated genes was normalized to that of RPLPO. Means \pm SD of $\geq 3$ technical replicates. H Representative images of serial sections from two human CRC patients stained for MIF and CD74 with different stabilization of MIF (MIF ${ }^{\text {high: }}$ : stabilized, MIF ${ }^{\text {low: }}$ unstabilized) and expression levels of CD74 (CD74 ${ }^{\text {high }}, \mathrm{CD}^{\mathrm{low}}$ ). Scale bars, $200 \mu \mathrm{mm}$. I, J Correlation between patients with stabilized MIF (MIF high) and CD74 levels (CD74 high, CD74 $4^{\text {low }}$ (I) and patients with unstabilized MIF (MIF Fow) and CD74 levels (CD74 $4^{\text {high }}$, CD74 $4^{\text {low }}$ ) (J) on overall survival of patients. Log-rank (Mantel-Cox) test for comparison of indicated groups. A, B, C, E, G $p$ values were calculated with Student's $t$ tests for comparison of indicated groups; ns $=$ not significant, ${ }^{*} p \leq 0.05,{ }^{* *} p \leq 0.01,{ }^{* * *} p \leq 0.001$.

recombinant MIF (rhMIF) in DLD-1 cells to mimic MIF secretion, also failed to activate ERK or angiogenic gene expression (Fig. 5D, E). Importantly, supplementation of both, MIF by rhMIF and CD74 by plasmid-based ectopic expression, lead to ERK activation and increased VEGFA and CXCL8/IL8 expression confirming that concomitant CD74 and secreted MIF are necessary for expression of angiogenic markers (Fig. 5F, G). To further investigate the MIF-CD74 axis, we performed clinical correlation studies based on MIF and CD74 expression levels of human CRC patients (Fig. 5H, I, J). Interestingly, simultaneous high levels of MIF and CD74 showed a trend for patient shortest survival (53.1 months) compared to stabilized MIF alone (71.4 months) (Fig. 5I). In contrast, CD74 status in patients with low MIF levels did not impact overall survival (Fig. 5J).

These findings underline the importance of MIF in cancer and support that MIF acts via CD74 in CRC. 


\section{MIF-driven CRC is vulnerable to Hsp90 inhibition}

Next, we asked whether constitutive MIF stabilization in CRC cells creates vulnerabilities that can be therapeutically targeted. Since MIF is stabilized by $\mathrm{Hsp} 90^{11}$, we used the pharmacological Hsp90 inhibitor 17AAG. When $\mathrm{Mif}^{+/+}$and $\mathrm{Mif}^{-/-}$mice reached a defined tumor burden, they were treated with 17AAG (Fig. 6A). Hsp90 inhibition reduced MIF protein levels in AOM/DSS tumors (Fig. 6B) and showed a trend for decreased tumor burden in $\mathrm{Mif}^{+/+}$mice (Fig. 6C-E). Differences were not statistically significant but showed a trend in $\mathrm{Mif}^{+/+}$mice (Fig. 6D, E, left panels). By contrast, Hsp90 inhibition in $\mathrm{Mif}^{-1-}$ mice failed to achieve tumor reduction (Fig. 6D, E, right panels).

To further support MIF as tumor-relevant Hsp90 client in CRC progression, we used genetically deleted MIF tumor organoid cultures. We observed a decreased growth in Mif-depleted organoids (Fig. 6F, G), further confirming, that MIF loss reduces tumor cell proliferation (Fig. 4H). Whether these growth defects arise from intracellular MIF functions and/or an MIF-CD74 axis remains elusive. Moreover, Vegfa expression was reduced in those organoids (Fig. 6G). To further support MIF as a tumor-relevant Hsp90 substrate in CRC, we analyzed these Mif-depleted organoids after treatment with 17AAG (Fig. 6G, H). Indeed, a Mif depletion led to a decreased susceptibility toward 17AAG treatment compared to Mifproficient organoids (Fig. $6 \mathrm{H}$ ). Furthermore, apoptotic markers such as cleaved caspase-3 and Parp were only upregulated after 17AAG treatment in Mif-proficient organoids, but not in Mif-deficient organoids (Fig. 6I).

These data support a relevant point: Hsp90 inhibition seems to stronger target CRC tumors with elevated MIF, although the HSP90 system stabilizes innumerable oncogenes. These findings support that MIF is a tumorrelevant Hsp90 substrate in CRC.

\section{MIF is a selective therapeutic target of Hsp90 inhibition in CRC-derived organoids}

To exploit further therapeutically targeting of stabilized MIF, we administered Hsp90 inhibitors to healthy epithelial/mucosal-derived and tumor-derived murine colonic organoids from the same AOM/DSS-induced mice (i.e., matched pairs). Since organoids derived from mice with a $129 \mathrm{~S} 1 / \mathrm{SvImJ}$ background failed to grow in vitro in our laboratory (Figure S5A), we used C57BL/6 mice. Observation of the organoid morphology and the subsequent quantifications showed higher levels of cell death after 17AAG in tumor-derived organoids, compared to the epithelial-derived organoids (Fig. 7A). Immunoblot analysis confirmed strong reduction of Mif levels especially after treatment with $500 \mathrm{nM}$ 17AAG (Fig. 7B). This prompted us to test Ganetespib and Onalespib, two clinically relevant second-generation HSP90 inhibitors that have been extensively tested in clinical trials and have a suitable toxicity profile ${ }^{58-61}$. Both inhibitors induced cell death to a far lesser extent in normal epithelial-derived organoids than in tumor-derived organoids (Fig. 7C) and showed promising specificity toward tumor organoids. Although Mif protein was degraded by Hsp90 inhibition in normal and tumorderived organoids treated with either inhibitor (Fig. 7D), only tumor-derived organoids were morphologically disrupted upon Hsp90 inhibition (Fig. 7C), indicating that MIF plays a tumorigenic role rather than an essential function in normal epithelial cells. Importantly, and in line with our findings, we confirmed the enhanced Mif levels in tumorderived organoids (Fig. 7B, D). Furthermore, in MIFexpressing patient-derived CRC organoids, Ganetespib markedly increased organoid death compared to that observed in the control organoids (Fig. 7E).

Therapeutic selectivity toward tumor cells plays an important role in therapy implementation. To further test whether Hsp90 inhibitors affect healthy tissues, we used organoids derived from the murine small intestine. Upon implementing the same treatment scheme as that used for colonic tumor-derived organoids, we discovered that 17AAG, Ganetespib, and Onalespib only exerted minor or no effects on the small intestine-derived organoids (Figure S5B). Indeed, Ganetespib failed to significantly degrade Mif protein in those organoids (Figure S5C), while another Hsp90 substrate, Stat3, was degraded. This confirms the selectivity of Hsp90 inhibition toward stabilized MIF in tumors. Even though immuno blot analysis also showed reduction of Mif levels after 17AAG treatment (Figure S5C), again depletion of unstabilized Mif in small intestinal organoids, did not impact morphology or survival of organoids (Figure S5B) as observed for normal colonic epithelia-derived organoids (Fig. 7A, B).

Thus, our findings highlight that MIF degradation via Hsp90 inhibition is a promising mechanism in CRC therapy. MIF acts not only as a critical driver in CRC but also as a selective target for Hsp90 inhibition in tumors.

\section{Discussion}

We used the immune-competent AOM/DSS mouse model, which mimics CRC progression in humans, to exploit the therapeutic potential of MIF. We demonstrated that MIF is specifically elevated in tumor cells and drives tumor growth in this acute colitis-associated ('sporadic') CRC model. Thus, in established tumors, stabilized MIF preferentially supports tumor-specific macrophage infiltration, vessel formation, and tumor cell proliferation.

Concomitantly, we also showed within this model that MIF regulates overall inflammatory signatures but especially during tumor initiation. Compared with $\mathrm{Mif}^{+/+}$ mice, $\mathrm{Mif}^{-1-}$ mice were protected against acute colitisassociated tumor initiation (Fig. 3), confirming the general function of MIF as a proinflammatory cytokine ${ }^{3,10}$. By 

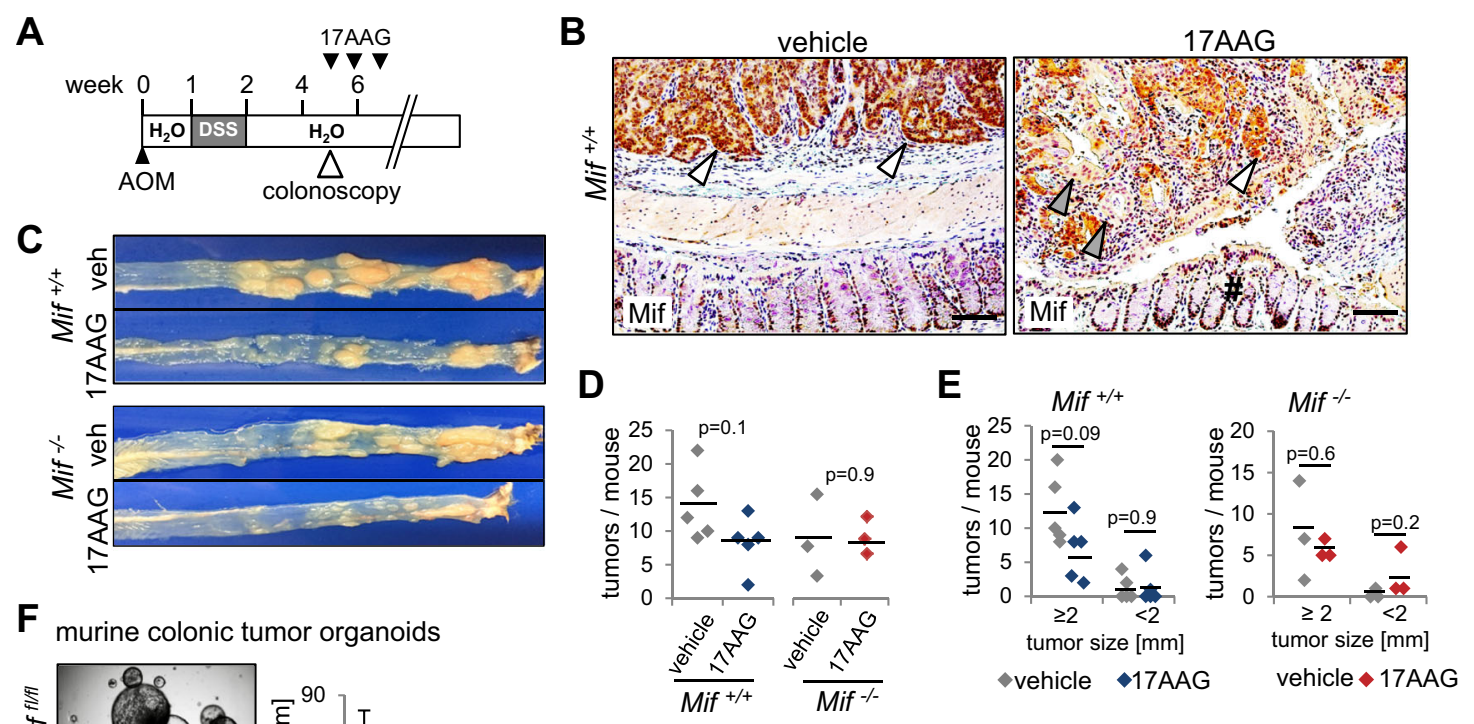

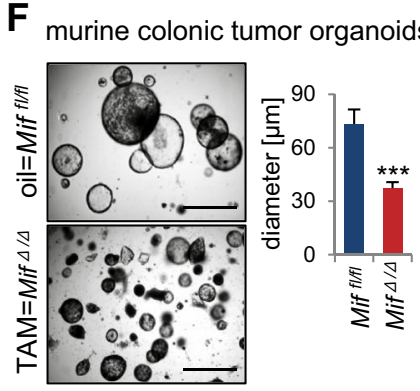

G

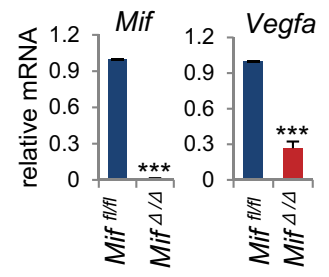

H

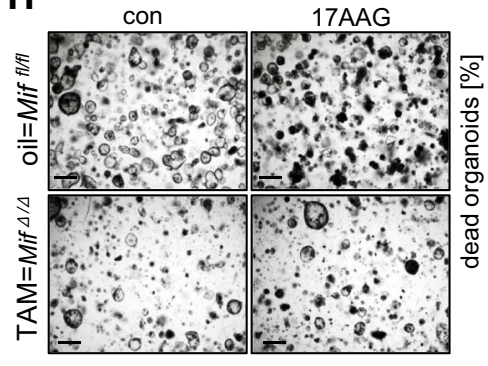

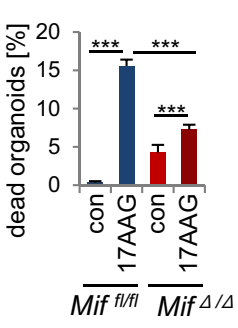

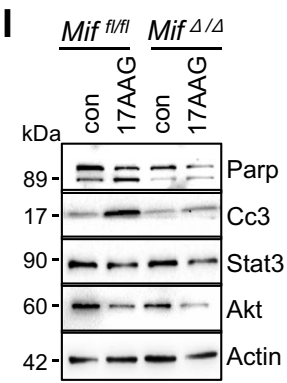

Fig. 6 MIF-driven CRC is vulnerable to Hsp90 inhibition. A Hsp90 inhibitor treatment timeline within the AOM/DSS mouse model. After tumors visualized via colonoscopy and scored as more than one S2/3 or three S1/2 tumors, mice were treated with the HSP90 inhibitor $17 A A G$ for 3 weeks. B Representative histological Mif staining of tumor-bearing colonic tissue from Mif ${ }^{+/+}$mice after 3 weeks of 17AAG or vehicle treatment. White arrows indicate tumor epithelial cells with elevated Mif levels. Gray arrows indicate tumor cells with degraded Mif. \#, normal epithelia of the next surrounding layer with low Mif levels. Scale bars, $100 \mu \mathrm{m}$. C Macroscopic overview of entire tumor-bearing colons from Mif ${ }^{+/+}$and Mif $^{-/-}$mice after $^{-1}$

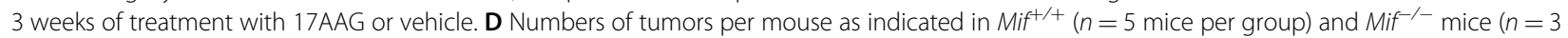
per group). Black lines, mean. $p$ values with ANOVA, Bonferroni's multiple comparison test, $p=0.212$. E Tumor sizes of mice from (D). Indicated groups, calculated with Student's $t$ test. F Assessment of organoid growth in Mif-depleted organoids. Mif ${ }^{\text {/lff }}$; villincreERT2 mice with a defined tumor burden were treated with Tamoxifen (TAM) or respective vehicle control to generate Mif-deficient CRC tumors (Mif P $^{\text {) }}$ to mimic a Mif epithelialspecific knockout. From those tumors, colonic tumor organoids were generated. Representative images and quantification of colonic tumor organoids ( $\mathrm{p} 5)$ derived from oil $\left(\mathrm{Mif}^{f / f f}\right)$ or TAM $\left(\mathrm{Mif}^{\mathrm{A} \Delta}\right)$ treated mice. (right) At least 11 images were quantified from 10 different gel domes. Diameter of organoids was measured using ImageJ. Scale bars, $200 \mu \mathrm{m}$. Means \pm SD from different images. G Expression analysis of Mif and Vegfa in Mif ${ }^{f / f l}$ or Mif $^{V \Delta}$ derived organoids. Via qRT-PCR, expression was co-normalized to Rplp0 and Hprt1. Means \pm SD of 4-5 technical replicates. $\mathbf{H}$ Imaging and quantification of organoid death of Mif-proficient (top) and Mif-deficient (bottom) organoids prepared as in (F) after 17AAG (500 nM) for 21 h. Scale bars, $200 \mu \mathrm{m}$. Quantifications based on organoid morphology. At least 5 images from $\geq 5$ gel domes ( $\times 4$ magnification each) per group were counted. The percentage of dead organoids was calculated to all organoids. Mean \pm SD from $\geq 6$ images. I Immunoblot of organoids of ( $\mathbf{H}$ ) at the endpoint of the experiment. Cc3 (cleaved caspase-3) and Parp (poly(ADP-ribose)-polymerase), apoptotic markers. Stat3 and Akt, further Hsp90 clients served as positive control for 17AAG functionality. Actin, loading control. F, G, H $p$ values, Student's t tests for indicated groups.

contrast, established Mif-deficient tumors did not show reductions in overall inflammation (Fig. 4); rather, only tumor-associated macrophages significantly infiltrated $\mathrm{Mif}^{+/+}$tumors. Thus, MIF seems to lose its overall proinflammatory function once CRC tumors are established. Proliferation, vessel formation and angiogenic cytokine expression were reduced in Mif-deficient tumors, an effect described previously $3,10,27,48$.

Studies showing that human tumor cells themselves are able to activate MAPK-mediated IL8 and VEGF 
A murine colon

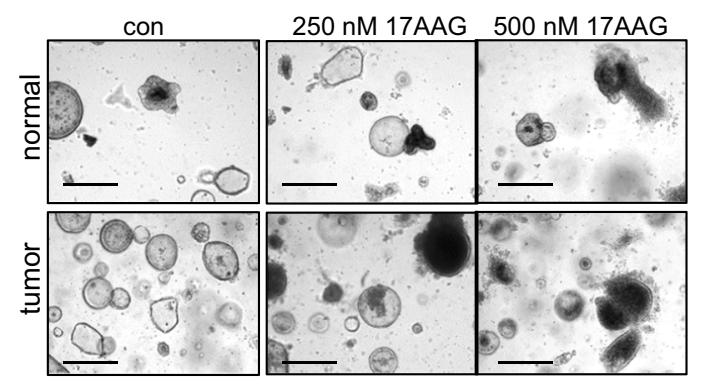

C murine colon

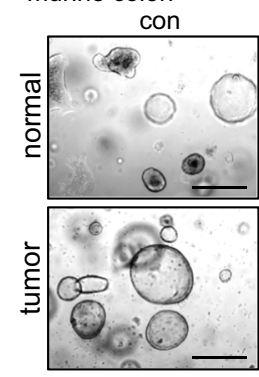

$30 \mathrm{nM}$ Ganet

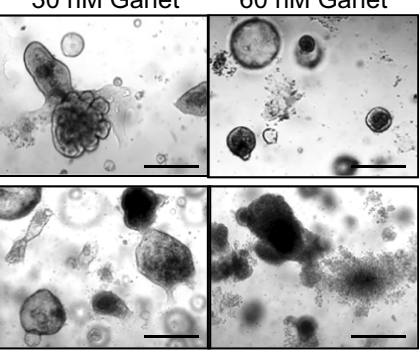

D

murine colon

$$
\text { normal }
$$

$\sum_{i=1} \sum_{i=1}$
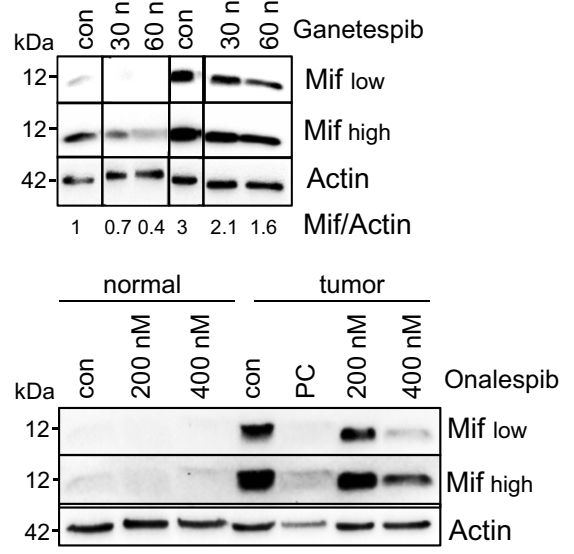

B

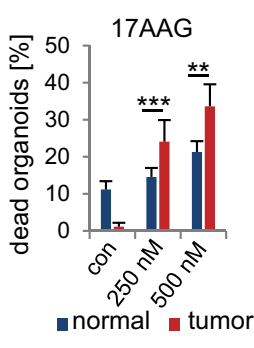

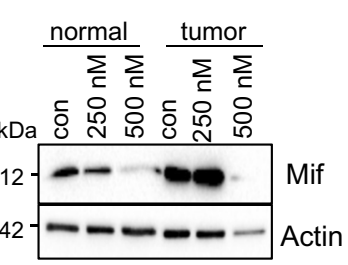

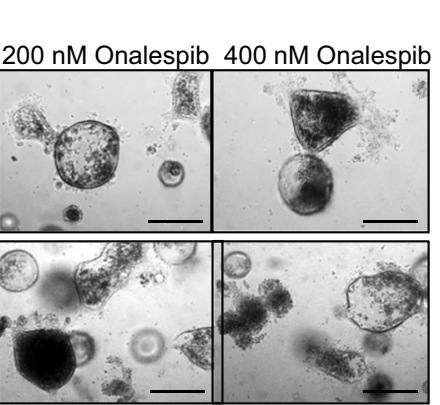

E

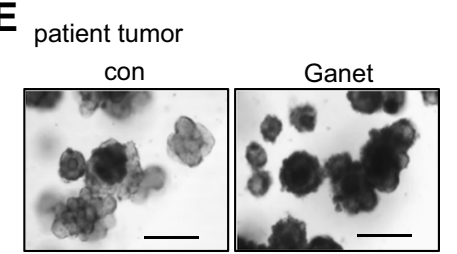

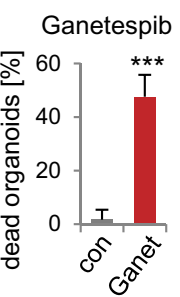

Fig. $7 \mathrm{MIF}$ is an actionable and selective therapeutic target via Hsp90 inhibition in CRC-derived organoids. A, C Therapeutic selectivity of Hsp90 inhibitors. Representative images show colonic organoids derived from matched pairs of pooled $\mathrm{Mif}^{+/+}$tumors or their adjacent epithelium ('normal'). The indicated organoids were treated with DMSO ('con'), 17AAG (A), Ganetespib ('Ganet'), or Onalespib (C) at the indicated concentrations for $21 \mathrm{~h}$. Scale bars, 200 um. (Right) Quantifications based on organoid morphology. At least 6 images from $\geq 5$ gel domes ( $x 4$ magnification each) per group were counted. For each group, number of dead organoids was calculated by the number of total organoids. The data are presented as the means \pm SD from different images. B, D Hsp90 inhibitor treatment as described in (A, C) of matched pairs. Immunoblot analysis was performed to evaluate Mif degradation. 'Mif high' and 'Mif low' indicate exposure times during signal acquisition. Actin, loading control. PC, positive control. Mif/ Actin, Mif expression ratios compared to Actin. E Representative images and quantification of organoids from patients with resectable CRC treated with $80 \mathrm{nM}$ Ganetespib for $21 \mathrm{~h}$. Scale bars, $200 \mu \mathrm{m}$. Quantification was performed as described in (A). Five images ( $\times 10$ magnification) per condition were quantified. Means \pm SD from different images. A, C, E Student's $t$ test was performed for comparison of indicated groups; ${ }^{*} p \leq 0.05,{ }^{* *} p \leq 0.01$, ${ }^{* * *} p \leq 0.001$.

expression by binding of MIF to its main receptor CD74 $7,12,27,28,35,53$ were confirmed within this study in human CRC cells (Fig. 5). Our data support that MIF can act in an autocrine MIF-CD74 manner in HCT116 CRC cells, resulting in accelerated expression of angiogenic factors. Furthermore, in DLD-1 cells, we supplemented recombinant MIF concomitantly with ectopic CD74 which mimics paracrine MIF-CD74 interactions to induce VEGF and IL8. In the in vivo CRC model, we assume that tumor epithelial cells do both, secrete MIF to recruit 
macrophages to the tumor (which consequently secrete angiogenic factors) (Fig. 4B); and provide an autocrine MIF-CD74 interaction to induce the MAPK-VEGF axis (Fig. 5B and F), albeit we have not specifically tested it in this study. However, reduced expression of VEGF in Mifdeficient organoids (Fig. 6G), support the idea, that tumor cells themselves contribute to VEGF expression. Nevertheless, MIF is known to act as chemokine on tumorspecific macrophage recruitment and/or macrophage polarization, and macrophages are known to secrete angiogenic factors, further promoting CRC tumorigenesis $^{44,55}$. In sum, tumor cells and tumor-associated macrophages might contribute to angiogenic factor expression but stabilized MIF in epithelial tumor cells provides the prerequisite for both scenarios.

To further test whether tumor epithelial cells with elevated MIF expression provide dual control over tumor growth, additional experimental models with inducible, tissue-specific Mif deletions are required. In principle, reduced chemotaxis of Mif $^{-/-}$macrophages ${ }^{62}$ or Mif-depleted fibroblasts within the tumor stroma ${ }^{63}$ might also contribute to tumor reduction in Mif $^{-1-}$ mice.

The co-expression of MIF and CD74 seems to be important in tumorigenesis (Fig. 5), and either MIF or CD74 alone might not be strong tumor biomarkers. Our CRC patient study (Fig. $5 \mathrm{H}-\mathrm{J}$ ) as well as patient studies of lung cancer and colon carcinomatosis indicate that MIF/ CD74 co-expression corresponds to an even worse prognosis $^{28,64}$. Moreover, a recent mouse study revealed a strong upregulation of CD74 during colonic inflammation, promoting mucosal healing, and epithelial tissue recovery by enhanced cell proliferation ${ }^{65}$. While this study confirms the importance of a MIF/CD74 co-existence in proliferation, it also clarified that a CD74 deficiency alone massively increases overall inflammation with a reduced recovery rate ${ }^{65}$. In contrast, MIF deletion or ablation alone protects against inflammation, demonstrated in experimental models of gastrointestinal inflammation ${ }^{66-68}$. Why a CD74 single deletion intensifies inflammation remains speculative ${ }^{65,69}$. One explanation might be altered macrophage recruitment. $\mathrm{MIF}^{-/-}$macrophages exhibited reduced overall chemotaxis compared to wild-type macrophages, whereas $\mathrm{CD}_{74^{-1-}}$ macrophages showed random chemokinesis $^{62}$, leading to an accelerated inflammatory response. Moreover, receptors often co-regulate each other, and after CD74 loss, MIF might increase its affinity to CXCR2 and/or CXCR4 receptors driving inflammation instead of proliferation and angiogenesis ${ }^{54,70}$. Dual roles for ligand-receptor complexes are becoming increasingly evident in the context of active inflammation and mucosal recovery ${ }^{69}$. In sum, MIF/CD74 co-expression might be the major predictor for tumor growth in CRC.
MIF is mainly stabilized in tumors but not stromal or inflammatory cells (Fig. 2). MIF stabilization occurs via binding to $\mathrm{Hsp} 90^{16}$, which offers therapeutic approaches to target cancer cells via Hsp90 inhibition. We showed for the first time that clinically relevant Hsp90 inhibitors decreased MIF levels in CRC and subsequently reduced tumor growth (Figs. 6 and 7). Given the plethora of known Hsp90stabilized oncogenes ${ }^{18}$, it is interesting to see that Hsp90mediated stabilization of MIF is critical for the survival of Mif-proficient murine colonic tumor-derived organoids (Fig. $6 \mathrm{H})$. MIF reduced tumor-derived organoids show a reduced antitumor response to Hsp90 interference compared to that in Mif-proficient organoids, indicating that MIF is an important Hsp90-stabilized protein in CRC. Moreover, Hsp90 interference provides therapeutic selectivity toward tumor cells (Fig. 7). Since Hsp90 inhibitors exhibit fundamental differences in action ${ }^{71}$, we focused on newly developed inhibitors such as Ganetespib and Onalespib.

In summary, since MIF stabilization is a crucial event, specifically in tumor cells, Hsp90 inhibition provides a potential approach to target MIF function in CRC. These findings support the tumor-promoting role of MIF in CRC and highlight the necessity to better understand the underlying MIF-induced tumorigenic mechanisms in CRC.

\section{Materials and methods \\ Patient samples}

Clinical samples (protein samples, RNA samples, PFAfixed paraffin-embedded sections, patient tissue for cultivation) were provided by the Department of General, Visceral and Pediatric Surgery of the University Medical Center Göttingen (UMG, Germany).

\section{Mouse models and genotyping}

Mouse experiments were approved by state (Niedersächsisches Landesamt für Verbraucherschutz und Lebensmittelsicherheit, LAVES, Germany) and institutional (Göttingen University Medical Center) committees, which ensured that all experiments conformed to the relevant regulatory standards. Constitutive Mif knockout in the $129 \mathrm{~S} 1 / \mathrm{SvImJ}$ background has been described in detail in ref. ${ }^{72}$. DNA isolation and genotyping were performed using DirectPCR lysis Reagent and OneTaq ${ }^{\circledR Q u i c k-L o a d}{ }^{\circledR}$ MasterMix. Genotyping primers are specified in Supplemental Table 1. Mifflox/flox mice in C57BL/6NCrl background were described in detail ${ }^{72,73}$; and were used for the development of murine organoids. In brief, to remove floxed MIF alleles from colonic epithelial tissue, we crossed Miflfl mice with villinCreERT2harboring mice to generate $M i^{f l f l}$; villincreERT2 transgenic mice. Mice were housed and handled under pathogen-free barrier conditions. 


\section{Murine CRC induction, colonoscopy, and treatment}

For experiments, randomly selected 10 -week-old female and male mice $(>20 \mathrm{~g}$ ) were used. For the induction of colorectal tumors, mice were treated with a single intraperitoneal injection of $10 \mathrm{mg} / \mathrm{kg}$ azoxymethane (AOM, Sigma-Aldrich) in $0.9 \%$ sodium chloride. After 1 week of rest, $1.5 \%$ (129S1/SvImJ background) or $2 \%$ (C57BL/6 background) DSS (MP-Biomedicals) was added to the drinking water for 6 consecutive days. Throughout the AOM/DSS phase, the body weights of the mice were continuously measured.

Five weeks after AOM induction, tumor development was monitored weekly by conducting a colonoscopy (Karl Storz $\mathrm{GmbH}$ ) on anesthetized mice (1.5-2\% isoflurane inhalation). Tumor sizes were determined according to the method described by Becker and Neurath ${ }^{41}$ based on the colonic luminal perimeter as follows: $\mathrm{S} 1=$ just detectable, $S 2=1 / 8$ of the lumen, $S 3=1 / 4$ of the lumen, $\mathrm{S} 4=1 / 2$ of the lumen, and $\mathrm{S} 5>1 / 2$ of the lumen. For analysis of established tumors, we chose an endpoint study design, terminating the experiment at 12 weeks after AOM treatment to avoid losing mice to extraneous reasons such as intestinal obstruction and anal prolapse.

For pharmacological Hsp90 inhibitor analysis, tumors were visualized and validated by colonoscopy. Reaching a defined tumor burden, at least one S2/3 tumor and at least three $S 1 / 2$ tumors when scored by colonoscopy, mice were treated with 17-allylamino, 17-demethoxygeldanamycin (17AAG, provided by the National Cancer Institute, NCI). Therefore, 17AAG was pre-dissolved in DMSO and further diluted in 10\% DMSO/18\% Kolliphor ${ }^{\circledR}$ RH40/3.6\% Dextrose (Sigma-Aldrich) in $\mathrm{H}_{2} \mathrm{O} .60 \mathrm{mg} / \mathrm{kg}$ of $17 \mathrm{AAG}$ or vehicle were given by intraperitoneal injection for 5 days per week for 3 consecutive weeks. During 17AAG treatment, tumors were weekly visualized and monitored by colonoscopy.

At endpoints, mice were euthanized and the entire large intestine was harvested, longitudinally opened, and displayed. Tumor numbers were counted and sizes were measured with an electronic caliper. For subsequent analysis, single tumor biopsies were taken. Each large intestine was 'swiss rolled', fixed in 3.7\% paraformaldehyde/PBS, processed for embedding and bisected. Both halves were placed into a mold for paraffin embedding.

For Mif depletion in vivo, AOM/DSS-treated Mifflfl mice were used for Tamoxifen (TAM, Sigma-Aldrich) or respective vehicle control (oil). Reaching a defined tumor burden, at least one $\mathrm{S} 2 / 3$ tumor and at least three $\mathrm{S} 1 / 2$ tumors when scored by colonoscopy, mice were treated for 5 consecutive days, followed by 2 days of rest and another 3 days TAM/oil treatment. Twelve days after TAM-end, mice were dissected, and organoids were prepared (see section above).
All animal experiments were carried out in full agreement with the guidelines outlined above.

\section{Human cell cultures, treatment, and transfection}

The human CRC cell line DLD-1 was cultured in RPMI 1640 medium, whereas HCT116 CRC cells were cultured in McCoy's 5A modified medium. Media were supplemented with $10 \%$ FBS, Penicillin-Streptomycin, and Lglutamine (RPMI 1640). Cell lines were cultured at $37^{\circ} \mathrm{C}$ and $5 \% \mathrm{CO}_{2}$ in a humidified atmosphere and were regularly tested for Mycoplasma contamination.

Knockdown of MIF or CD74 was achieved by siRNA transfection using Lipofectamine ${ }^{\mathrm{TM}} 3000$ reagent according to the manufacturer's instructions. All siRNAs were purchased from Ambion and used according their guidelines; the sequences are listed in Supplemental Table 2. CD74 overexpression in DLD-1 cells was performed using Lipofectamine $^{\mathrm{TM}} 3000$ transfection reagent. In brief, $24 \mathrm{~h}$ after cell seeding, DLD-1 cells were cotransfected with $0.5 \mu \mathrm{g}$ of GFP-containing plasmid and $1.5 \mu \mathrm{g}$ of either pcDNA3.1CD74 expression plasmid ${ }^{56}$ or the corresponding pcDNA3.1/V5-His-TOPO control plasmid. Forty-eight hours post-transfection, cells were treated with recombinant human MIF as indicated.

\section{HEK293T cell media conditioning for organoid culture medium}

HEK293T cells expressing murine R-spondin-1 and Noggin or Wnt3a were cultivated in DMEM supplemented with $10 \%$ FBS, Penicillin-Streptomycin and Sodium Pyruvate in a humidified atmosphere at $37^{\circ} \mathrm{C}$ with $5 \% \mathrm{CO}_{2}$. For HEK293T-mR-spondin-1 Zeocin and for HEK293TmNoggin Geneticin were added to the medium during cultivation and expansion. For conditioning, medium was replaced by advanced DMEM/F12 medium supplemented with GlutaMAX ${ }^{\mathrm{TM}}$, Penicillin-Streptomycin, and $10 \mathrm{mM}$ HEPES, and cells were cultivated for 1 week. For murine Rspondin-1-containing and Noggin-containing media, batch quality was examined using Dot-blot analysis. Murine colonic organoid culture medium contains advanced DMEM/F12 medium supplemented with 50\% conditioned Wnt3a medium, 20\% conditioned Noggin medium, 10\% conditioned R-spondin-1 medium, N-2, B-27, $3.4 \mu \mathrm{g} / \mathrm{mL}$ ROCK inhibitor, $5 \mu \mathrm{M}$ CHIR 99021, $500 \mathrm{nM}$ A83-01, $10 \mathrm{mM}$ Nicotinamide, $80 \mu \mathrm{M}$ N-Acetyl-L-Cysteine, and $200 \mathrm{ng} / \mathrm{mL}$ rmEGF.

\section{Preparation and cultivation of colonic and small intestinal organoids}

Tumor-harboring large intestines of C57BL/6 mice were harvested. Three to four tumors per mouse and in parallel, parts of the normal epithelium were biopsied from the same mouse allowing generation of matched organoid pairs. Normal epithelial tissue was cut, washed, 
and incubated in $4 \mathrm{mM}$ EDTA/PBS for $30 \mathrm{~min}$ on ice. Pieces were thoroughly, mechanically dissociated in PBS. Tumor samples were digested with $2 \mathrm{mg} / \mathrm{mL}$ Collagenase type-I solution at $37^{\circ} \mathrm{C}$ for $30 \mathrm{~min}$. Normal crypts and tumor fragments were filtered using cell strainers. Cell pellets were washed, resuspended in cold Matrigel, and droplet-plated allowing Matrigel polymerization at $37^{\circ} \mathrm{C}$ for $30 \mathrm{~min}$. Organoids-containing domes were covered with organoid culture medium, cultivated at humidified $37{ }^{\circ} \mathrm{C}$ with $5 \% \mathrm{CO}_{2}$. Medium was exchanged every 2 to 3 days. Organoids splitting was performed once a week. For passaging, organoids were manually disrupted in icecold PBS, and cultured as described above.

Small intestinal tract starting from jejunum to the end of ileum were prepared from C57BL/6 mice, incubated in $5 \mathrm{mM}$ EDTA/PBS for $30 \mathrm{~min}$ on ice, washed, and mechanically dissociated. Crypts were resuspended in cold Matrigel and cultured as colonic organoids, but with small intestinal organoid medium containing advanced DMEM/ F12 supplemented with $20 \%$ conditioned mNoggin medium, $10 \%$ conditioned R-spondin-1 medium, N-2, B-27, $80 \mu \mathrm{M}$ $\mathrm{N}$-Acetyl-L-Cysteine, $50 \mathrm{ng} / \mathrm{mL}$ rmEGF.

\section{Organoid treatments and morphological quantification}

Experiment with matched pairs of normal epitheliaderived and tumor-derived colonic organoids, murine small intestinal organoids, and human organoids were performed between passage 2-7. HSP90 inhibitors 17AAG (National Cancer Institute, NCI), Onalespib and Ganetespib (Synta Pharmaceuticals) were dissolved in DMSO and used as indicated. For quantification of treatment response, light microscopy images of $\geq 5$ Matrigel domes were taken from each condition. The amount of images was dependent on size and culture density as indicated. Based on morphology, dead organoids were defined as organoids with a partial or complete loss of outer epithelial barrier leading to disruption into clumps of dead cells or separation of dead cells ${ }^{74}$. The percentage of dead organoids was calculated relative to the total amount of organoids per image. For dead organoid quantification and measurement of organoid diameter ImageJ was used. For analysis of organoid lysates, Matrigel domes were disrupted using ice-cold PBS. Suspension was centrifuged and organoid-containing pellets were further washed and incubated with Cell Recovery solution (Corning) for complete removal of Matrigel. Organoids were resuspended in standard RIPA buffer for protein lysates and in TRIZOL for RNA isolation.

\section{Histological analysis}

Immunohistological stainings were performed with standardized protocols for formalin-fixed paraffin-embedded (FFPE) tissues. Following primary antibodies were used: MIF (Sigma-Aldrich, HPA003868), CD74 (Sigma-Aldrich,
HPA010592), phospho-histone H2A.X (Ser139, Cell Signaling, \#9718), Ki67 (Abcam, ab15580), Cluster of differentiation 31 (CD31 (SZ31), Dianova, DIA-310), Cluster of differentiation 3 (CD3 [SP7], Abcam, ab16669), Forkheadbox protein p3 (FoxP3, Abcam, ab54501), Myeloperoxidase (MPO, R\&D Systems, AF3667). For CD68, two different antibodies were used to double-check staining (Abcam, ab53444 and eBioscience ${ }^{\mathrm{TM}}$, 14-0681-82). For detection of primary antibodies from rabbit and rat, ImmPRESS ${ }^{\mathrm{TM}}$ Reagent anti-Rabbit IgG and ImmPressTM Reagent antiRat IgG (both Vector Laboratories) were used. For antibodies from goat, the $A B C$ detection system was used, entailing a biotinylated goat/sheep antibody (GE Healthcare) and ExtrAvidin ${ }^{\circledR}$-Peroxidase (Sigma-Aldrich). As substrate for the horseradish peroxidases served 3,3'-Diaminobenzidine tetrahydrochloride (DAB, Roth). Counterstain of the nuclei was achieved using Mayers Hämalaun solution (Merck). Alexa Fluor®594-coupled secondary antibody was used as detection system for immunofluorescence with DAPI (Sigma-Aldrich) as counterstain for nuclei. Images were taken using a standard microscope (Carl Zeiss AG) with the ZEN imaging program from Zeiss. Figures were further prepared using Adobe Photoshop software. For quantification of staining, samples were blinded and positively stained cells were counted manually using CellCounter function of ImageJ. Percentage of epithelial Ki67-positive cells was determined relative to the total number of epithelial cells. For staining of CD31, CD68, CD3, FoxP3, and MPO, the number of positive cells was counted per image.

Hämalaun \& Eosin G stained sections were used to determine the inflammatory score. The inflammatory score is based on morphological changes (grade of damage) of the tissue due to immune cell infiltration and epithelial layer disruption. Grade $0=$ factor 0 , no infiltration of immune cells, normal distribution of epithelia and amount of goblet cells; grade $1=$ factor 1 , minor infiltration of immune cells, epithelia is still intact and minor changes in goblet cell number; grade $2=$ factor 2 , moderate infiltration of inflammatory cells, epithelia is partly damaged and reduced number of goblet cells; grade $3=$ factor 3 , massive infiltration of immune cells, complete disruption/loss of epithelia and loss of goblet cells. For calculation, amount of tissue in percentage with respective grade of tissue damage was multiplied with the corresponding factor (factor 0-3). The obtained percentages were summed, resulting in a value for the inflammatory score (minimum 0-maximum 300) for each mouse. To ensure unbiased quantification, the inflammatory score was individually determined by one scientist and one pathologist.

TUNEL staining was used to detect DNA-strand breaks occurring during apoptotic cell death in established tumors. TUNEL reaction mix (Sigma-Aldrich) consists of TUNEL 
enzyme solution and TUNEL label mix. The assay was performed according to manufactures guidelines. DAPI served as counterstaining, slides were mounted with Fluorescent Mounting Medium (DakoCytomation).

\section{Quantitative immunohistochemistry on colon cancer patient samples}

Section of a tissue micro array (TMA) for primary colonic tumors was kindly provided by the Department of Pathology of the University Medical Center Göttingen (UMG, Germany). According to described standard protocols for immunohistochemistry (see above), TMAs were stained for MIF (Sigma-Aldrich, HPA003868) and CD74 (Sigma-Aldrich, HPA010592). For CD74 staining tumors with more than $10 \%$ strongly positive stained cells or more than $40 \%$ overall stained cells with lower intensity were graded as high $\left(\mathrm{CD} 74^{\text {high }}\right)$. For MIF staining, biopsis with high intensity in more than $70 \%$ of cells were graded as MIF high . Biopsis with moderate or low intensity were graded as MIF $^{\text {low }}$.

\section{Immunoblot analysis}

For Whole lysates from human CRC cell lines and murine organoids were made with standard RIPA buffer (1\% sodium deoxycholate, $10 \mathrm{mM}$ EDTA, $1 \%$ Triton $\mathrm{X}$ 100, 0.1\% SDS, $150 \mathrm{mM} \mathrm{NaCl,} 20 \mathrm{mM}$ Tris- $\mathrm{HCl} \mathrm{pH} 7.5$, cOmplete $^{\mathrm{TM}}$ mini protease inhibitor cocktail and phosphatase inhibitor mix consisting of $2 \mathrm{mM}$ Imidazol, $1 \mathrm{mM}$ sodium orthovanadate, and $1 \mathrm{mM}$ sodium fluoride) was used. For protein extraction from human and murine samples, tissues were minced, lysed in RIPA buffer, and further processed by sonication. For determination of protein concentrations using BCA protein assay (Pierce), samples were centrifuged and supernatants were collected. Equal protein concentrations were separated by SDS gel electrophoresis and transferred onto nitrocellulose membranes (Amersham). After blocking with 5\% milk (Roth), membranes were incubated with the following antibodies: MIF and CD74 (both Sigma-Aldrich); CDK4 and $\beta$-Actin (both Abcam); HSC70 [B-6], total AKT, phospho-AKT [D9E], phospho-p44/42 MAPK (ERK1/2), phospho-p38 MAPK [3D7], p38 MAPK, cleaved caspase-3, and PARP (all Cell Signaling); VEGF, STAT3, and ERK (all Santa Cruz). All primary antibodies were detected with HRP-conjugated secondary antibodies. Development of the signal was performed using Immobilion western chemiluminescent HRP substrate (Millipore/Merck) or Clarity Max $^{\mathrm{TM}}$ Western ECL Substrate (BioRad). Detailed antibody information in Supplemental Table 2.

\section{Quantitative real-time PCR (qRT-PCR)}

RNA from human cell lines and colonic tissues and tumors was isolated using Trizol reagent (Invitrogen) according to manufacturer guidelines. Tissues and tumor pieces were shredded using a homogenizer (T10 basic ULTRA-TURRAX). After reverse transcription (M-MuLV Reverse Transcriptase from NEB) of equal amounts of mRNA, quantitative real-time PCR analysis was performed using a qPCR MasterMix (72 mM Tris- $\mathrm{HCl} \mathrm{pH}$ 8.8 (Roth), $19 \mathrm{mM}$ (NH4)2SO4 (Roth), 0.01\% Tween-20 (AppliChem), $3 \mathrm{mM} \mathrm{MgCl}_{2}$, (Sigma-Aldrich), 1:80,000 SYBR Green (Invitrogen), 0.24 mM dNTPs, (dATP, dCTP, dGTP, dTTP, all dNTPs from Primetech), 19 U/ml Taqpolymerase (Primetech), 0.24\% Triton X-100 (AppliChem), $300 \mathrm{mM}$ Trehalose (Roth). Used primers are listed in Supplemental Table 1. For gene analysis, at least two different cDNAs (technical replicates) were used for qRTPCR runs from one biological replicate. Biological replicates are independent experiments.

\section{Quantification and statistical analysis}

Statistics of each experiment such as number of animals, number of tumors, biological replicates, technical replicates, precision measures (mean and $\pm \mathrm{SD}$ ), and the statistical tests used for significance are provided in the figures and figure legends.

Densitometric measurements for quantification of immunoblot bands were done with the gel analysis software Image $\mathrm{Lab}^{\mathrm{TM}}$ (BioRad) and normalized to loading controls.

Pearson correlation factor $\mathrm{R}$ was used for analysis of immunohistochemical correlation studies on CRC tissue. GraphPad Prism was used for analysis of Kaplan-Meier survival curves with the Log-rank (Mantel-Cox) test.

The following designations for levels of significance were used within this manuscript: ${ }^{*} p \leq 0.05$; ${ }^{* * *} p \leq 0.01$; $* * * * 0.001 ;$ ns, not significant.

\section{Acknowledgements \\ We thank Jennifer Appelhans (Pathology, Göttingen), Joshua Blume, and Tamara Isermann (Molecular Oncology, Göttingen) for their technical assistance and Robyn Laura Kosinsky (Clinic for General, Visceral and Pediatric Surgery, Göttingen) for providing the HEK293T Wnt3a cells.}

\section{Funding}

R.S.-H. and L.K. are supported by the Deutsche Forschungsgemeinschaft (DFG) (SCHUH-3160/3-1), R.S.-H. by a Dorothea Schlözer Fellowship (University Medical Center Göttingen) and the Else Kröner-Fresenius Stiftung. Open Access funding enabled and organized by Projekt DEAL.

\section{Author details}

${ }^{1}$ Institute of Molecular Oncology, University Medical Center Göttingen, Göttingen, Germany. '2Department of General, Visceral, and Pediatric Surgery, University Medical Center Göttingen, Göttingen, Germany. ${ }^{3}$ Institute of Pathology, University Medical Center Göttingen, Göttingen, Germany.

${ }^{4}$ Departments of Medicine, Pathology, and Epidemiology \& Public Health, Yale School of Medicine and Yale Cancer Center, New Haven, CT, USA

\section{Author contributions}

Conceptualization: R.S.-H. and L.K.; methodology: R.S.-H., L.K., T.D.O., D.W., L.-C. C., N.W., and R.B.; acquisition of data: all authors; analysis and interpretation of 
data: R.S.-H., L.K., D.W., and L.-C. C.; writing original draft: R.S.-H. and L.K.; writing review, and editing: all authors; final approval: all authors; funding acquisition: R.S.-H.; supervision: R.S.-H.

\section{Ethics approval}

Patient samples (protein samples, RNA samples, PFA-fixed paraffin-embedded sections, patient tissue for cultivation) were provided by the Department of General, Visceral and Pediatric Surgery of the University Medical Center Göttingen (UMG, Germany) with approval from the ethics committee of UMG for the collection of CRC samples (approval numbers 9/8/08 and 25/3/17).

\section{Conflict of interest}

The authors declare that they have no conflict of interest.

\section{Publisher's note}

Springer Nature remains neutral with regard to jurisdictional claims in published maps and institutional affiliations.

Supplementary information The online version contains supplementary material available at https://doi.org/10.1038/s41419-021-03426-z.

Received: 11 December 2020 Revised: 2 January 2021 Accepted: 7 January 2021

Published online: 04 February 2021

\section{References}

1. Bucala, R. \& Donnelly, S. C. Macrophage migration inhibitory factor: a probable link between inflammation and cancer. Immunity 26, 281-285 (2007).

2. Conroy, H., Mawhinney, L. \& Donnelly, S. C. Inflammation and cancer: macrophage migration inhibitory factor (MIF) - the potential missing link. QJM 103, 831-836 (2010)

3. Gordon-Weeks, A. N., Lim, S. Y., Yuzhalin, A. E., Jones, K. \& Muschel, R. Macrophage migration inhibitory factor: a key cytokine and therapeutic target in colon cancer. Cytokine Growth Factor Rev. 26, 451-461 (2015).

4. Jankauskas, S. S., Wong, D. W. L., Bucala, R., Djudjaj, S. \& Boor, P. Evolving complexity of MIF signaling. Cell Signal 57, 76-88 (2019).

5. Mitchell, R. A. \& Yaddanapudi, K. Stromal-dependent tumor promotion by MIF family members. Cell Signal 26, 2969-2978 (2014).

6. Simons, D. et al. Hypoxia-induced endothelial secretion of macrophage migration inhibitory factor and role in endothelial progenitor cell recruitment. J. Cell Mol. Med. 15, 668-678 (2011).

7. Amin, M. A. et al. Migration inhibitory factor mediates angiogenesis via mitogen-activated protein kinase and phosphatidylinositol kinase. Circ. Res. 93 321-329 (2003)

8. Asare, Y., Schmitt, M. \& Bernhagen, J. The vascular biology of macrophage migration inhibitory factor (MIF). Expression and effects in inflammation, atherogenesis and angiogenesis. Thromb. Haemost. 109, 391-398 (2013).

9. Lee, J. P. et al. Loss of autophagy enhances MIF/macrophage migration inhibitory factor release by macrophages. Autophagy 12, 907-916 (2016).

10. O'Reilly, C., Doroudian, M., Mawhinney, L. \& Donnelly, S. C. Targeting MIF in cancer: therapeutic strategies, current developments, and future opportunities. Med. Res. Rev. 36, 440-460 (2016).

11. Schulz, R. et al. Inhibiting the HSP90 chaperone destabilizes macrophage migration inhibitory factor and thereby inhibits breast tumor progression. $J$. Exp. Med. 209, 275-289 (2012)

12. He, X. X. et al. Macrophage migration inhibitory factor promotes colorectal cancer. Mol. Med. 15, 1-10 (2009).

13. Hira, E. et al. Overexpression of macrophage migration inhibitory factor induces angiogenesis and deteriorates prognosis after radical resection for hepatocellular carcinoma. Cancer 103, 588-598 (2005).

14. Maaser, C., Eckmann, L., Paesold, G., Kim, H. S. \& Kagnoff, M. F. Ubiquitous production of macrophage migration inhibitory factor by human gastric and intestinal epithelium. Gastroenterology 122, 667-680 (2002).

15. Morris, K. T., Nofchissey, R. A., Pinchuk, I. V. \& Beswick, E. J. Chronic macrophage migration inhibitory factor exposure induces mesenchymal epithelial transition and promotes gastric and colon cancers. PLoS ONE 9, e98656 (2014).
16. Schulz, R., Dobbelstein, M. \& Moll, U. M. HSP90 inhibitor antagonizing MIF: The specifics of pleiotropic cancer drug candidates. Oncoimmunology $\mathbf{1}$ 1425-1426 (2012)

17. Gomez-Pastor, R., Burchfiel, E. T. \& Thiele, D. J. Regulation of heat shock transcription factors and their roles in physiology and disease. Nat. Rev. Mol. Cell Biol. 19, 4-19 (2018).

18. Schopf, F. H., Biebl, M. M. \& Buchner, J. The HSP90 chaperone machinery. Nat. Rev. Mol. Cell Biol. 18, 345-360 (2017).

19. Chen, W. T. et al. Identification of biomarkers to improve diagnostic sensitivity of sporadic colorectal cancer in patients with low preoperative serum carcinoembryonic antigen by clinical proteomic analysis. Clin. Chim. Acta $\mathbf{4 1 2}$ 636-641 (2011).

20. Lee, $\mathrm{H}$. et al. Macrophage migration inhibitory factor may be used as an early diagnostic marker in colorectal carcinomas. Am. J. Clin. Pathol. 129, 772-779 (2008).

21. Croner, L. J. et al. Discovery and validation of a colorectal cancer classifier in a new blood test with improved performance for high-risk subjects. Clin. Proteom. 14, 28 (2017)

22. Yasasever, $\mathrm{V}$. et al. Macrophage migration inhibitory factor in cancer. Cancer Invest. 25, 715-719 (2007).

23. Marmol, I., Sanchez-de-Diego, C., Pradilla Dieste, A., Cerrada, E. \& Rodriguez Yoldi, M. J. Colorectal carcinoma: a general overview and future perspectives in colorectal cancer. Int. J. Mol. Sci. 18, https://doi.org/10.3390/ijms18010197 (2017).

24. Ogawa, $H$. et al. An antibody for macrophage migration inhibitory factor suppresses tumour growth and inhibits tumour-associated angiogenesis. Cytokine 12, 309-314 (2000).

25. Shin, H. N., Moon, H. H. \& Ku, J. L. Stromal cell-derived factor-1alpha and macrophage migration-inhibitory factor induce metastatic behavior in CXCR4expressing colon cancer cells. Int. J. Mol. Med. 30, 1537-1543 (2012).

26. Sun, B. et al. Induction of macrophage migration inhibitory factor by lysophosphatidic acid: relevance to tumor growth and angiogenesis. Int. J. Mol. Med. 12, 633-641 (2003).

27. Wilson, J. M. et al. Macrophage migration inhibitory factor promotes intestinal tumorigenesis. Gastroenterology 129, 1485-1503 (2005).

28. Bozzi, F. et al. MIF/CD74 axis is a target for novel therapies in colon carcinomatosis. J. Exp. Clin. Cancer Res. 36, 16 (2017).

29. Cheon, S. K. et al. Macrophage migration inhibitory factor promotes resistance to MEK blockade in KRAS mutant colorectal cancer cells. Mol. Oncol. 12, 1398-1409 (2018).

30. Russo, R. et al. Macrophage migration inhibitory factor is a molecular determinant of the anti-EGFR monoclonal antibody cetuximab resistance in human colorectal cancer cells. Cancers 11, https:/doi.org/10.3390/cancers11101430 (2019).

31. Yao, K. et al. Macrophage migration inhibitory factor is a determinant of hypoxia-induced apoptosis in colon cancer cell lines. Clin. Cancer Res. 11, 7264-7272 (2005).

32. Pacheco-Fernandez, T. et al. Corrigendum to "Macrophage migration inhibitory factor promotes the interaction between the tumor, macrophages, and $\mathrm{T}$ cells to regulate the progression of chemically induced colitis-associated colorectal cancer". Mediators Inflamm. 2020, 2195341, https://doi.org/10.1155/ 2020/2195341 (2020)

33. Hussain, F. et al. Human anti-macrophage migration inhibitory factor antibodies inhibit growth of human prostate cancer cells in vitro and in vivo. Mol Cancer Ther. 12, 1223-1234 (2013).

34. Martin, J. et al. Macrophage migration inhibitory factor (MIF) plays a critical role in pathogenesis of ultraviolet-B (UVB) -induced nonmelanoma skin cancer (NMSC). FASEB J. 23, 720-730 (2009).

35. Choudhary, S. et al. Macrophage migratory inhibitory factor promotes bladder cancer progression via increasing proliferation and angiogenesis. Carcinogenesis 34, 2891-2899 (2013)

36. Talos, F., Mena, P., Fingerle-Rowson, G., Moll, U. \& Petrenko, O. MIF loss impairs Myc-induced lymphomagenesis. Cell Death Differ. 12 1319-1328 (2005)

37. Reinart, N. et al. Delayed development of chronic lymphocytic leukemia in the absence of macrophage migration inhibitory factor. Blood 121, 812-821 (2013)

38. Nemajerova, A, Mena, P., Fingerle-Rowson, G., Moll, U. M. \& Petrenko, O. Impaired DNA damage checkpoint response in MIF-deficient mice. EMBO J. 26, 987-997 (2007) 
39. De Robertis, M. et al. The AOM/DSS murine model for the study of colon carcinogenesis: from pathways to diagnosis and therapy studies. J. Carcinog. 10, 9 (2011)

40. Tanaka, T. et al. A novel inflammation-related mouse colon carcinogenesis model induced by azoxymethane and dextran sodium sulfate. Cancer Sci. $\mathbf{9 4}$ 965-973 (2003).

41. Becker, C., Fantini, M. C. \& Neurath, M. F. High resolution colonoscopy in live mice. Nat. Protoc. 1, 2900-2904 (2006).

42. Becker, C. et al. In vivo imaging of colitis and colon cancer development in mice using high resolution chromoendoscopy. Gut 54, 950-954 (2005).

43. Hudson, J. D. et al. A proinflammatory cytokine inhibits p53 tumor suppressor activity. J. Exp. Med. 190, 1375-1382 (1999).

44. Yaddanapudi, $\mathrm{K}$. et al. Control of tumor-associated macrophage alternative activation by macrophage migration inhibitory factor. J. Immunol. 190 2984-2993 (2013).

45. Owen, J. L. \& Mohamadzadeh, M. Macrophages and chemokines as mediators of angiogenesis. Front Physiol. 4, 159 (2013).

46. Mehrad, B., Keane, M. P. \& Strieter, R. M. Chemokines as mediators of angiogenesis. Thromb. Haemost. 97, 755-762 (2007).

47. Corliss, B. A., Azimi, M. S., Munson, J. M., Peirce, S. M. \& Murfee, W. L. Macrophages: an inflammatory link between angiogenesis and lymphangiogenesis. Microcirculation 23, 95-121 (2016).

48. Nishihira, J. et al. Macrophage migration inhibitory factor (MIF): its potential role in tumor growth and tumor-associated angiogenesis. Ann. N. Y Acad. Sci. 995, 171-182 (2003)

49. Lue, $\mathrm{H}$. et al. Macrophage migration inhibitory factor (MIF) promotes cell survival by activation of the Akt pathway and role for CSN5/JAB1 in the control of autocrine MIF activity. Oncogene 26, 5046-5059 (2007).

50. Oliveira, C. S. et al. Macrophage migration inhibitory factor engages PI3K/Akt signalling and is a prognostic factor in metastatic melanoma. BMC Cancer $\mathbf{1 4}$ 630 (2014).

51. Mitchell, R. A. et al. Macrophage migration inhibitory factor (MIF) sustains macrophage proinflammatory function by inhibiting p53: regulatory role in the innate immune response. Proc. Natl Acad. Sci. USA 99, 345-350 (2002).

52. Hanahan, D. \& Weinberg, R. A. Hallmarks of cancer: the next generation. Cell 144, 646-674 (2011)

53. Leng, L. et al. MIF signal transduction initiated by binding to CD74. J. Exp. Med. 197, 1467-1476 (2003).

54. Zernecke, A., Bernhagen, J. \& Weber, C. Macrophage migration inhibitory factor in cardiovascular disease. Circulation 117, 1594-1602 (2008).

55. Yaddanapudi, K. \& Mitchell, R. A. In MIF Family Cytokines in Innate Immunity and Homeostasis (eds Richard Bucala \& Jürgen Bernhagen) 59-76 (Springer International Publishing, 2017).

56. Shi, $X$. et al. CD44 is the signaling component of the macrophage migration inhibitory factor-CD74 receptor complex. Immunity 25, 595-606 (2006).

57. Hu, C. T. et al. MIF, secreted by human hepatic sinusoidal endothelial cells, promotes chemotaxis and outgrowth of colorectal cancer in liver prometastasis. Oncotarget 6, 22410-22423 (2015).
58. Jhaveri, K. \& Modi, S. Ganetespib: research and clinical development. Onco Targets Ther. 8, 1849-1858, (2015).

59. Alexandrova, E. M. et al. Improving survival by exploiting tumour dependence on stabilized mutant p53 for treatment. Nature 523, 352-356 (2015).

60. Do, K. et al. Phase I study of the heat shock protein 90 (Hsp90) inhibitor onalespib (AT13387) administered on a daily for 2 consecutive days per week dosing schedule in patients with advanced solid tumors. Invest. N. Drugs 33 921-930 (2015)

61. Graham, B. et al. The heat shock protein 90 inhibitor, AT13387, displays a long duration of action in vitro and in vivo in non-small cell lung cancer. Cancer Sci. 103, 522-527 (2012).

62. Fan, $\mathrm{H}$. et al. Macrophage migration inhibitory factor and CD74 regulate macrophage chemotactic responses via MAPK and Rho GTPase. J. Immunol. 186, 4915-4924 (2011).

63. Petrenko, O., Fingerle-Rowson, G., Peng, T., Mitchell, R. A. \& Metz, C. N. Macrophage migration inhibitory factor deficiency is associated with altered cell growth and reduced susceptibility to Ras-mediated transformation. J. Biol. Chem. 278, 11078-11085 (2003).

64. McClelland, M., Zhao, L., Carskadon, S. \& Arenberg, D. Expression of CD74, the receptor for macrophage migration inhibitory factor, in non-small cell lung cancer. Am. J. Pathol. 174, 638-646 (2009).

65. Farr, L. et al. CD74 Signaling Links Inflammation to Intestinal Epithelial Cell Regeneration and Promotes Mucosal Healing. Cell Mol. Gastroenterol. Hepatol., https://doi.org/10.1016/j.jcmgh.2020.01.009 (2020).

66. de Jong, Y. P. et al. Development of chronic colitis is dependent on the cytokine MIF. Nat. Immunol. 2, 1061-1066 (2001).

67. Ohkawara, T. et al. Amelioration of dextran sulfate sodium-induced colitis by anti-macrophage migration inhibitory factor antibody in mice. Gastroenterology 123, 256-270 (2002).

68. Wong, B. L. et al. Essential role for macrophage migration inhibitory factor in gastritis induced by Helicobacter pylori. Am. J. Pathol. 174, 1319-1328 (2009).

69. Hoffmann, A., Zwissler, L. C., El Bounkari, O. \& Bernhagen, J. Studying the promigratory effects of MIF. Methods Mol. Biol. 2080, 1-18 (2020).

70. Schwartz, V. et al. A functional heteromeric MIF receptor formed by CD74 and CXCR4. FEBS Lett. 583, 2749-2757 (2009).

71. Neckers, L. et al. Methods to validate Hsp90 inhibitor specificity, to identify offtarget effects, and to rethink approaches for further clinical development. Cell Stress Chaperones 23, 467-482 (2018).

72. Fingerle-Rowson, $\mathrm{G}$. et al. The p53-dependent effects of macrophage migration inhibitory factor revealed by gene targeting. Proc. Natl Acad. Sci. USA 100 9354-9359 (2003).

73. Brocks, T. et al. Macrophage migration inhibitory factor protects from nonmelanoma epidermal tumors by regulating the number of antigen-presenting cells in skin. FASEB J. 31, 526-543 (2017)

74. Grabinger, T. et al. Ex vivo culture of intestinal crypt organoids as a mode system for assessing cell death induction in intestinal epithelial cells and enteropathy. Cell Death Dis. 5, e1228 (2014). 


\section{Supplemental material}
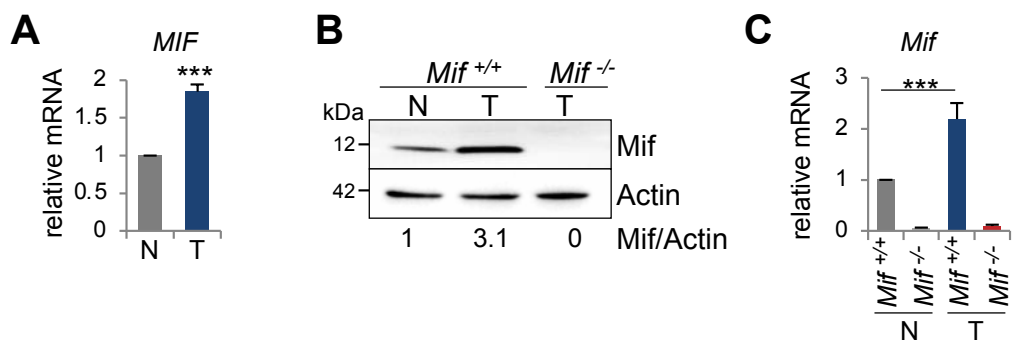

Supp Figure 1

Figure S1: Related to Figure 2. MIF levels are elevated in colorectal cancer cells. (A) Pooled cases of single patients (P1-P3), including tumor samples ('T') and their adjacent normal epithelium ('N') ( $n=3$ each). qRT-PCRs normalized to RPLPO mRNA. MIF expression in tumor samples was calculated relative to respective adjacent epithelium. Mean \pm SD of 3 technical replicates in duplicates. (B) Mif protein level in pooled samples of tumors ('T') and normal epithelium ('N') of Mift/+ mice. Mif/- tumors serve as negative staining control. Mif ratios (Mif/Actin) were calculated by densitometry, normalized to loading control, relative to $\mathrm{Mif}^{+/+}$epithelium ('N'). (C) Relative Mif mRNA levels of pooled normal ('N', $\mathrm{n} \geq 2$ ) and tumor (' $T$ ', $\mathrm{n}=6$ ) samples from $\mathrm{Mif}^{+/+}$and $\mathrm{Mif}^{--}$mice. Mean $\pm \mathrm{SD}$ of 5 technical replicates in duplicates. (A, C) Student's t test used for comparison of indicated groups: ${ }^{* *} p \leq 0.001$. 
Klemke et al. Cell Death and Disease (2021)

Supplemental material
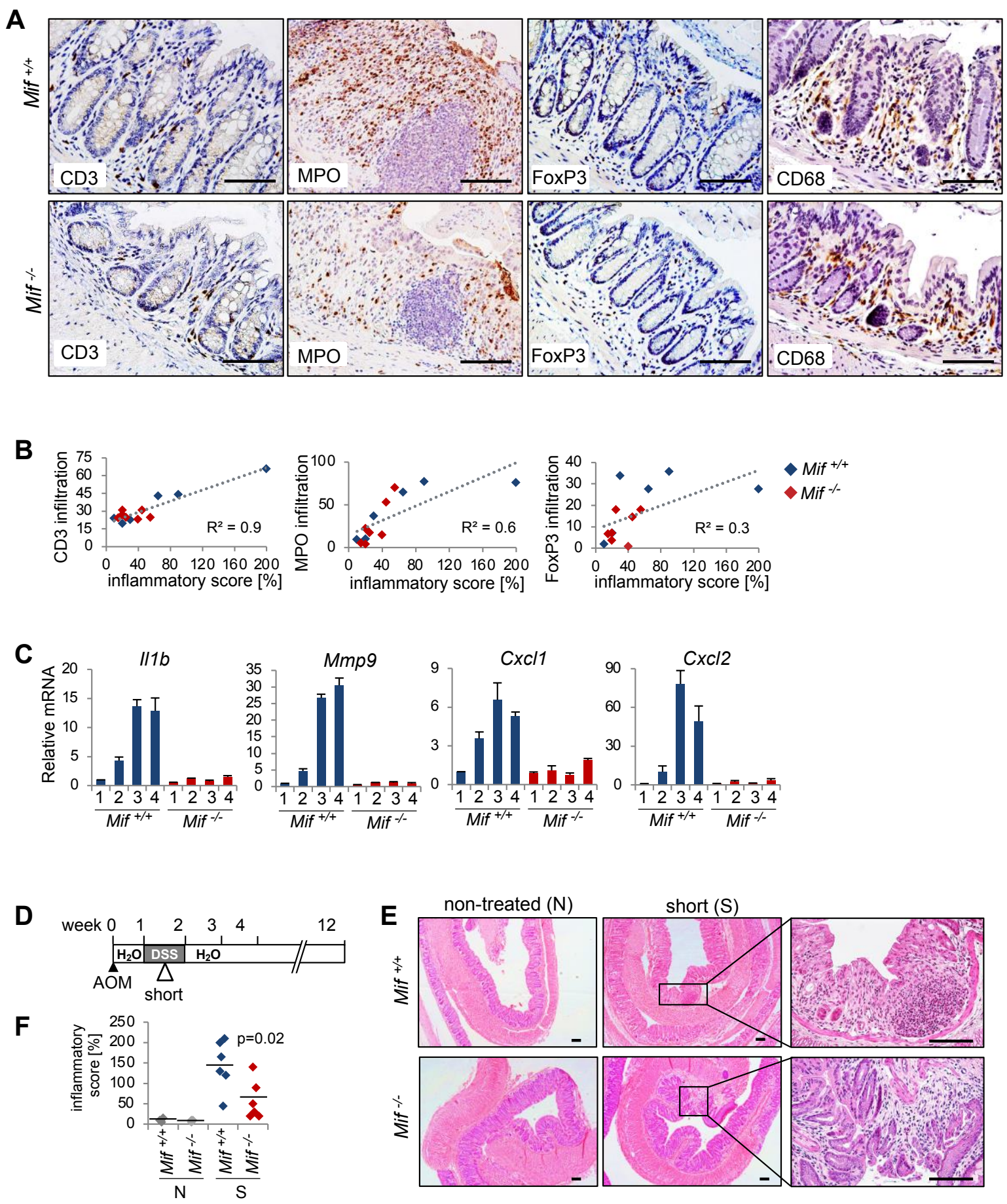

Supp Fig 2 


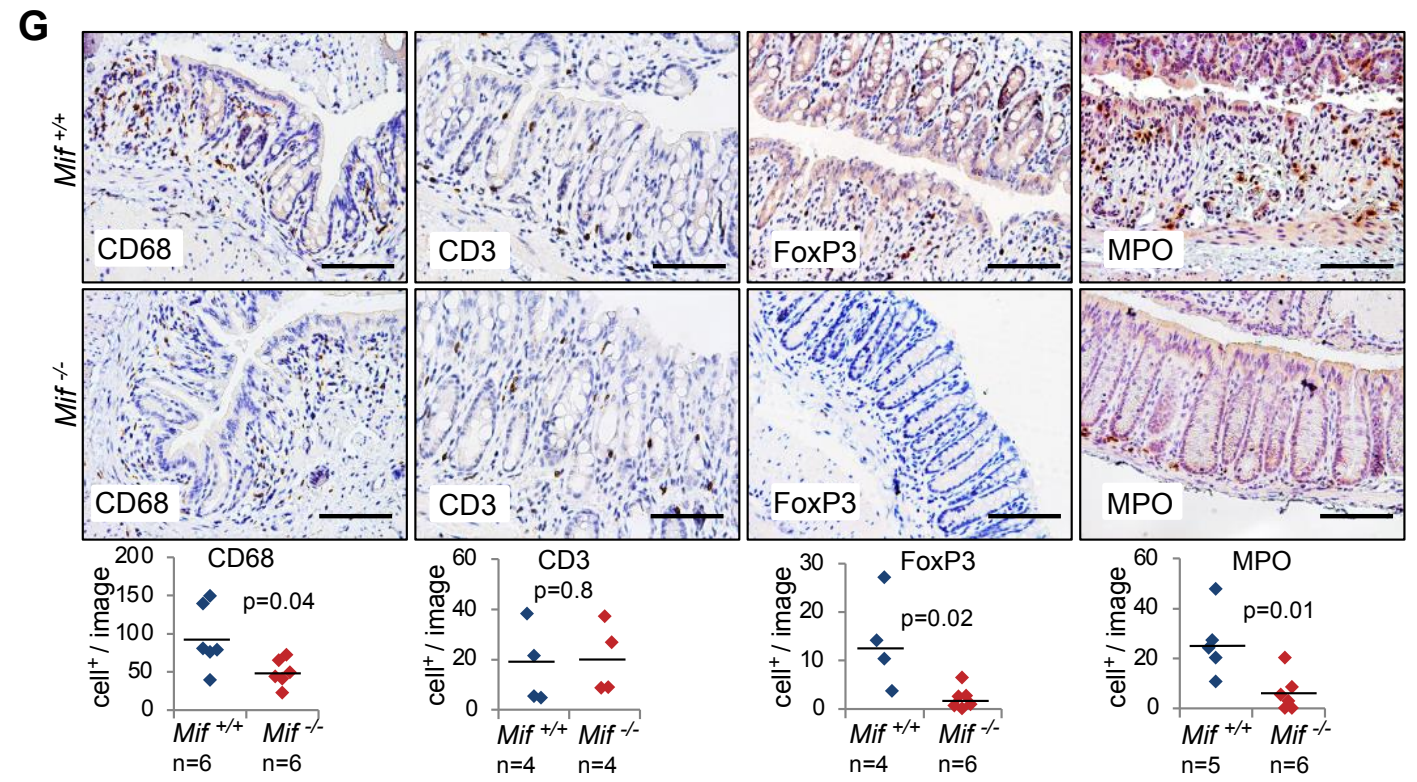

H post-AOM treatment
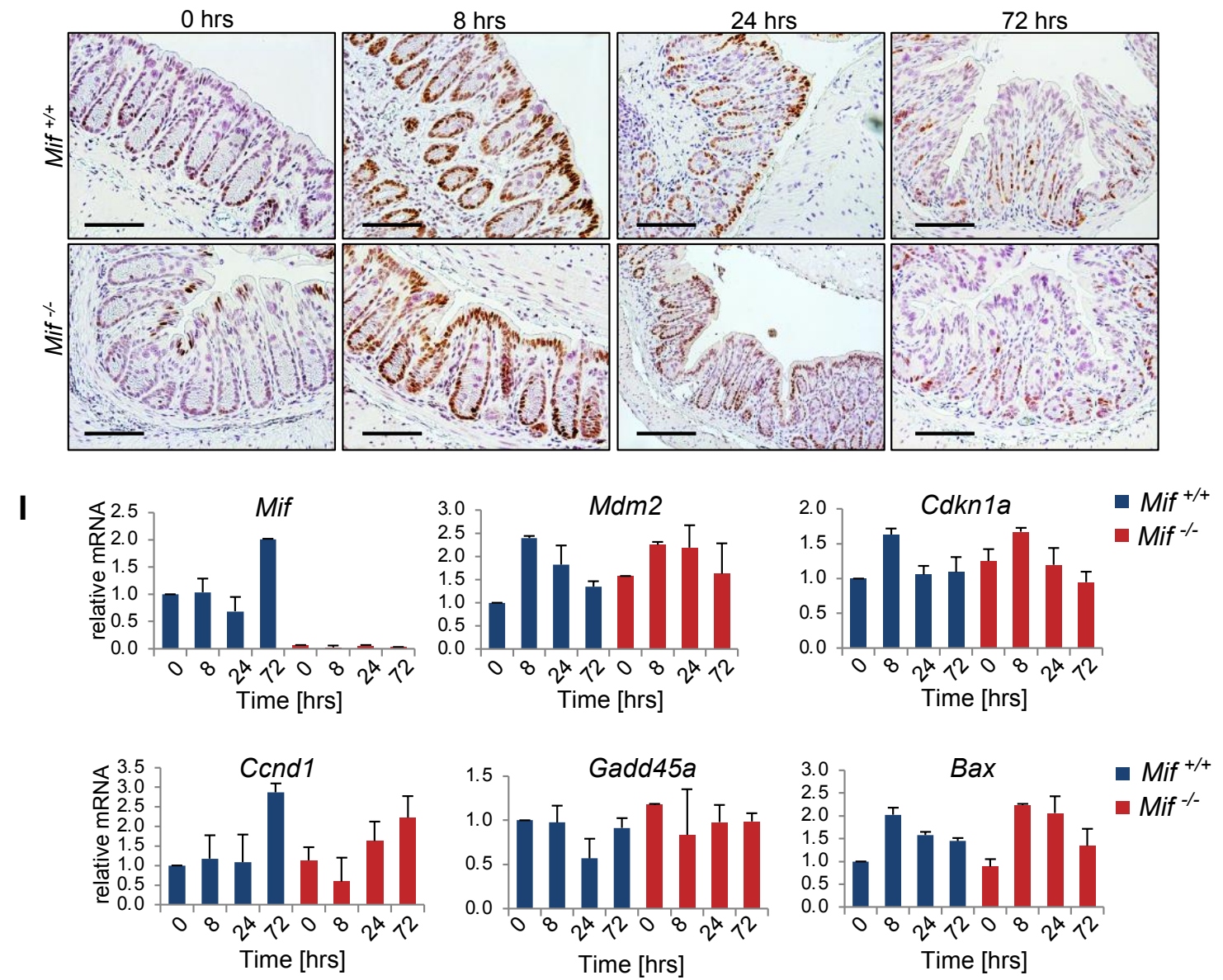

Supp Fig 2 ext 
Klemke et al. Cell Death and Disease (2021)

Supplemental material

Figure S2: Related to Figure 3. A MIF deletion protects mice from inflammation-associated cancer initiation. (A) Histological staining of colonic tissues 8 days after DSS (recovery period) of indicated genotypes. Inflammatory marker CD68 (Cluster of Differentiation 68) for monocytes/macrophages, CD3 (Cluster of Differentiation 3) for T-lymphocytes, FoxP3 (Foxhead-box-protein P3) for regulatory T-cells and MPO (Myeloperoxidase) for neutrophils/granulocytes. Scale bars, $100 \mu \mathrm{m}$. (B) Correlation between the inflammatory score of $\mathrm{Mif}^{+/+}$and $\mathrm{Mif}^{/-}$mice (Figure 3B) and the respective quantification of infiltrating immune cells (CD3, MPO and FoxP3 staining) in the recovery group from (Figure 3C). CD3, MPO and FoxP3 with $\mathrm{Mif}^{+/+} \mathrm{n}=6$ and $\mathrm{Mif}^{/-} \mathrm{n}=7$. R, Pearson correlation factor. (C) mRNA expression of representative cytokines of individual mice from recovery group (8 days post-DSS) from single samples (4 mice per group). Via qRT-PCR, expression levels were normalized to those of RplpO. Means \pm SD of $\geq 2$ technical replicates in duplicates. (D) Treatment scheme of the AOM/DSS colorectal cancer mouse model. Mice of the 'short' group were dissected at day 3 after starting DSS administration. (E) Representative H\&E staining of colonic tissues of indicated genotype at day 3 after DSS start (short, 'S') or in untreated control tissue ('N'). Scale bar, $100 \mu \mathrm{m}$. (F) The inflammatory score of the 'short' DSS and control groups was assessed based on H\&E stained tissue morphology in (E). Non-treated control $n=3$ mice per group, short $n=6$ mice per group. Black line, mean. $p$ value with Student's $t$ test. $(G)$ Representative histology of inflammatory cells (CD68, CD3, FoxP3, MPO staining) in $\mathrm{Mif}^{+/+}$and $\mathrm{Mif}^{\prime-}$ colonic tissues at day 3 after DSS start. Scale bars, $100 \mu \mathrm{m}$. For quantifications of histological staining, 4-5 images (area=40x magnification) per mouse were counted for positive stained stromal cells. $n$, number of mice. Black line, mean. $p$ values were calculated via Student's $t$ test. (H) A MIF loss is dispensable for AOM-induced DNA damage response. Representative immunohistological phosphoHistone H2A.X staining after the initial AOM injection at indicated time points. Scale bars, $100 \mu \mathrm{m}$. (I) A MIF loss does not impair the AOM-induced p53 response. mRNA level of Mif and WTp53 target genes of indicated groups at different time points after a single AOM injection. Single colonic tissues of indicated time points and genotypes were pooled ( $\mathrm{n} \geq 3$ mice per group). qRT-PCRs normalized to Rp/p0 mRNA. Mean \pm SD of 2 technical replicates, pipetted in duplicates. 
Klemke et al. Cell Death and Disease (2021)

Supplemental material

\section{A}

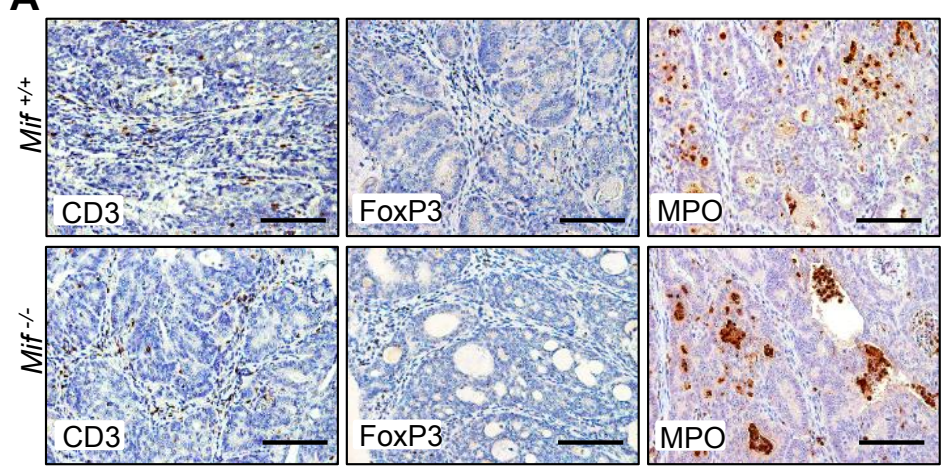

C

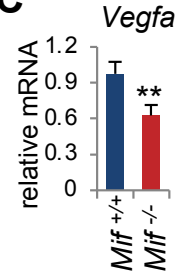

D

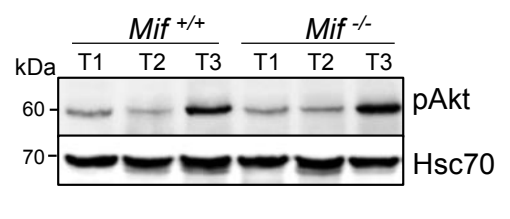

B

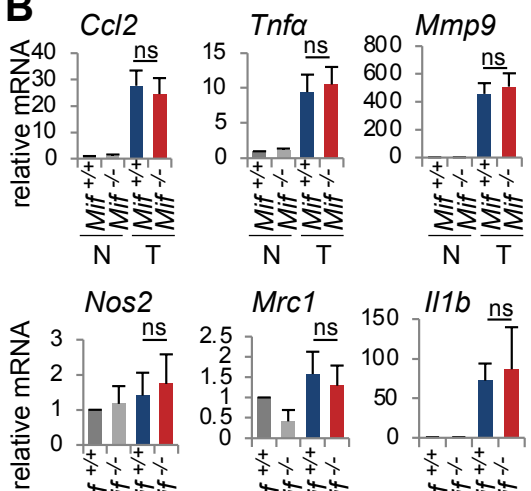

管

N

$\frac{2}{\mathrm{~N}}$

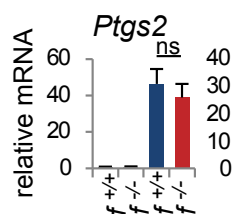

$\mathrm{Cxc} / 1 \quad \mathrm{Cxc} / 2$

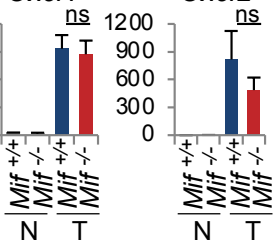

$\mathbf{F}$

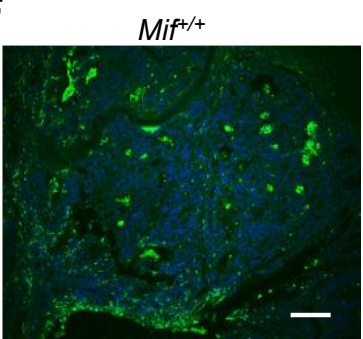

H
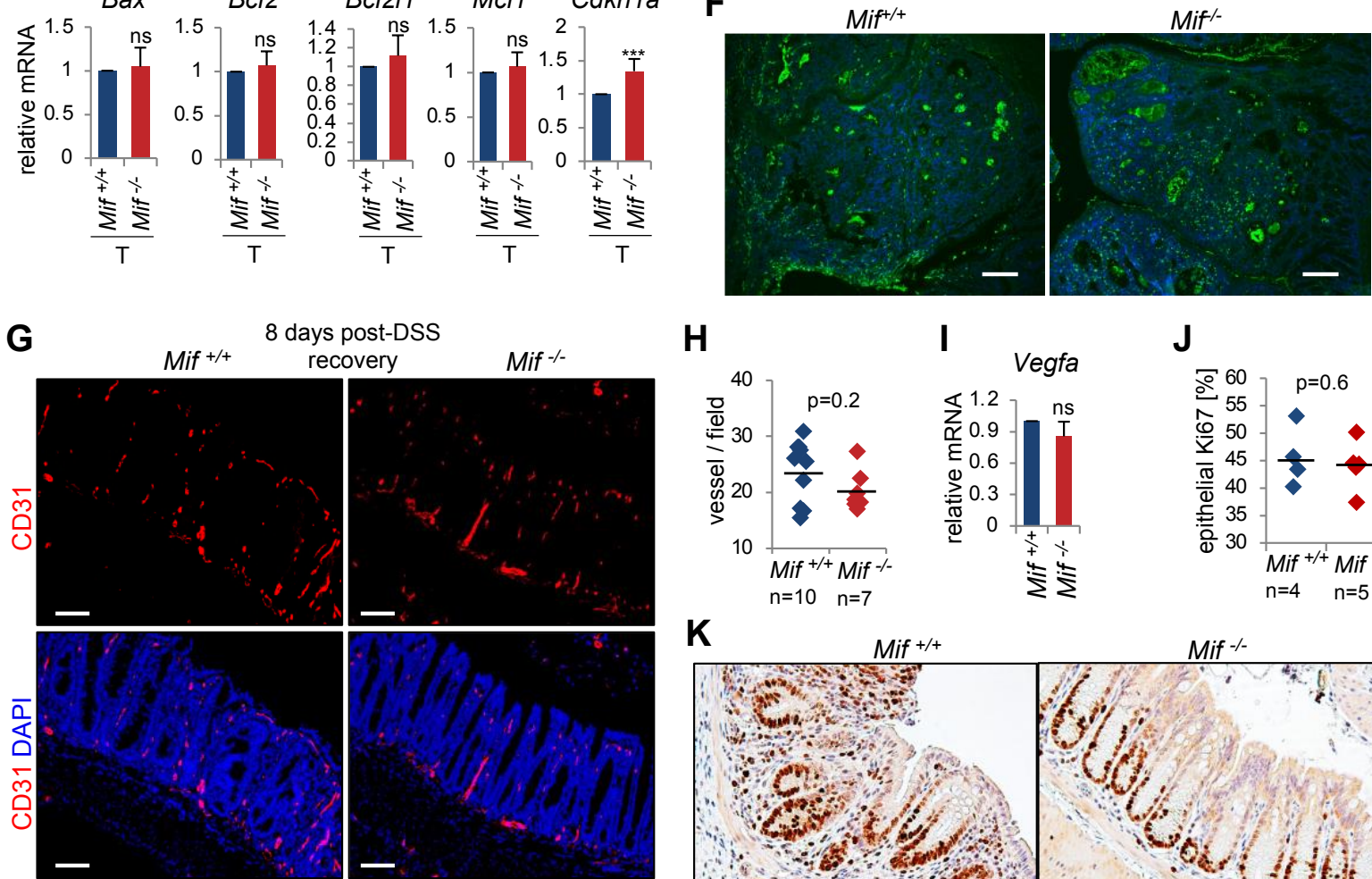

K

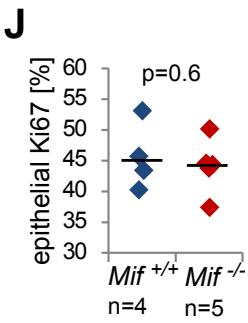

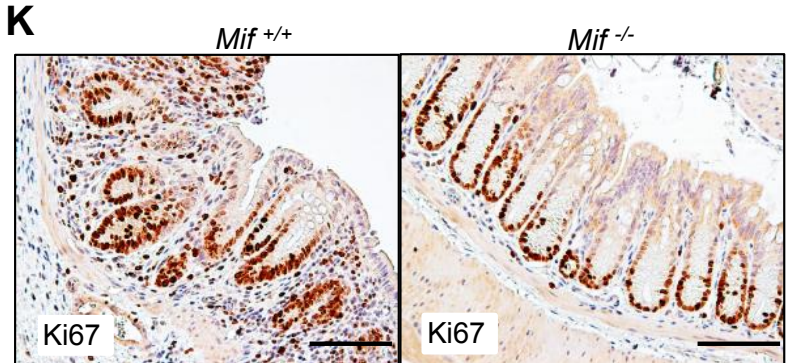

Supp Figure 3 
Klemke et al. Cell Death and Disease (2021)

Supplemental material

Figure S3: Related to Figure 4. MIF supports CRC tumor growth and macrophage infiltration without affecting overall inflammation. (A) Representative histology of CD3, FoxP3 and MPO staining of $\mathrm{Mif}^{+/+}$and Mif ${ }^{/-}$tumors. Scale bars, $100 \mu \mathrm{m}$. (B) mRNA expression of inflammatory genes in tumors ('T') and nontreated control tissues ('N') of indicated genotypes. Single colonic tissues ( $\mathrm{n} \geq 2)$ or single tumors $(n \geq 6)$ of indicated genotypes were pooled. qRT-PCR, expression levels were normalized to those of Rplpo. Means \pm SD of 3-4 technical replicates in duplicate. Student's $t$ test. (C) Relative expression of Vegfa, averaged from single tumor samples (7-8 mice) (Figure 4C). Means \pm SD of 4 technical replicates. (D) Phospho (p)-Akt level of single tumors (T1-T3) of $\mathrm{Mif}^{+/+}$and Mif/- mice. Hsc70, loading control. (E) Apoptotic gene expression of indicated tumors. Pooled single tumors ( $n \geq 6$ ). qRTPCR normalized to Rplp0 mRNA. Mean \pm SD of $\geq 5$ technical replicates in duplicates. $p$ value with Student's t test. ${ }^{* *} \mathrm{p} \leq 0.001$; ns, not significant. (F) TUNEL staining in $\mathrm{Mif}^{+/+}$and Mif/- tumors at 12 weeks post-AOM. Scale bars, $100 \mu \mathrm{m}$. (G, H) Representative immunofluorescence of $\mathrm{Mif}^{+/+}$and $\mathrm{Mif}^{\text {/- }}$ colonic tissue at 8 days post-DSS (recovery group), for CD31 (red) and DAPI (blue). Scale bars, $100 \mu \mathrm{m}$. Quantification of tumor vessel fragment density from $(G)$. At least 6 images (area $=40 x$ magnification) per colonic tissue were counted and calculated. Black lines, mean. $n$, number of mice. $p$ value with Student's t test. (I) Relative expression of Vegfa in recovering Mif ${ }^{+/+}$and Mif/ colonic tissue (pool of 4-5 mice per group). Via qRT-PCR expression was calculated relative to those of Rplp0. Mean \pm SD of 3 technical replicates in duplicates. p value with Student's t test. ns, not significant. (J, K) Quantification of epithelial Ki67 staining $(\mathrm{J})$ and representative histology of recovering $\mathrm{Mif}^{+/+}$and $\mathrm{Mif}^{-}$colonic tissue at 8 days post-DSS (K). Ki67 positivity was performed using 6 images per mouse. n, number of mice. Normalization of positive epithelial cells to total number of epithelial cells. $p$ value with Student's $t$ test. Scale bars, $100 \mu \mathrm{m}$. 
Klemke et al. Cell Death and Disease (2021)

Supplemental material
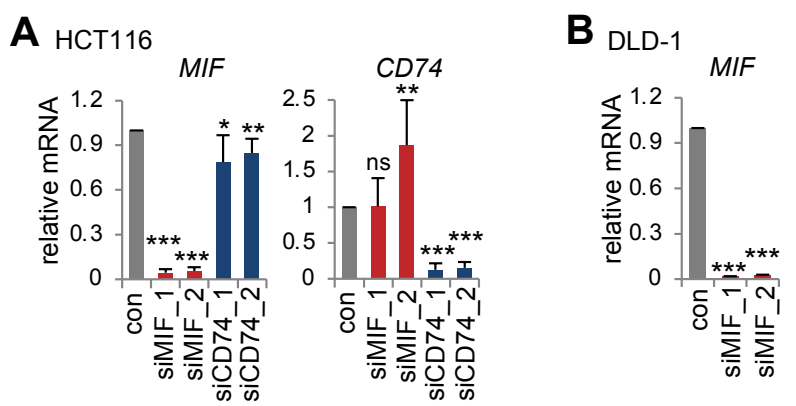

Supp Figure 4

Figure S4: Related to Figure 5. Knockdown efficiency of MIF and CD74 by small interfering RNA (siRNA) in CRC epithelial cells. (A, B) Expression of MIF and CD74 in HCT116 (A) and DLD-1 (B) cells normalized to RPLPO or HPRT1 respectively for evaluation of knockdown efficiency after $72 \mathrm{hrs}$ siRNA transfection against MIF, CD74 or respective scrambled control ('con'). Mean \pm SD of 5-6 technical replicates in duplicates from 2 biological replicates. Student's t test: ns=not significant, ${ }^{*} p \leq 0.05$; ${ }^{* *} \mathrm{p} \leq 0.01 ;{ }^{* *} \mathrm{p} \leq 0.001$. 
Klemke et al. Cell Death and Disease (2021)

Supplemental material
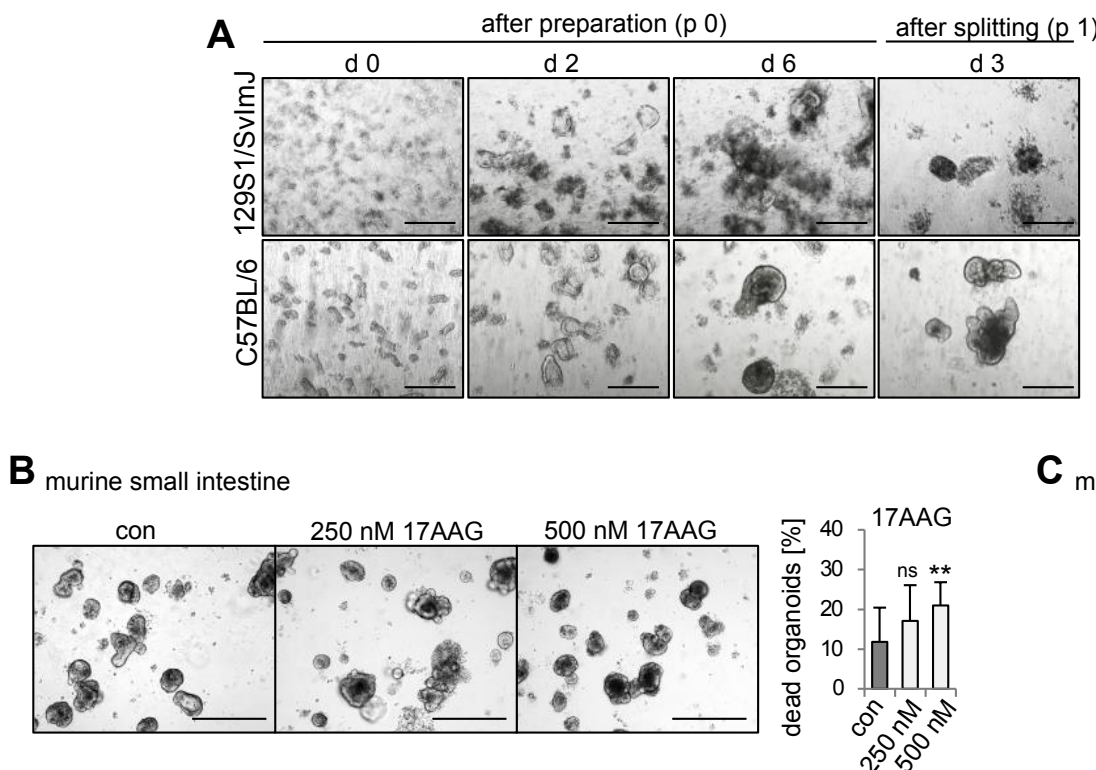

C murine small intestine
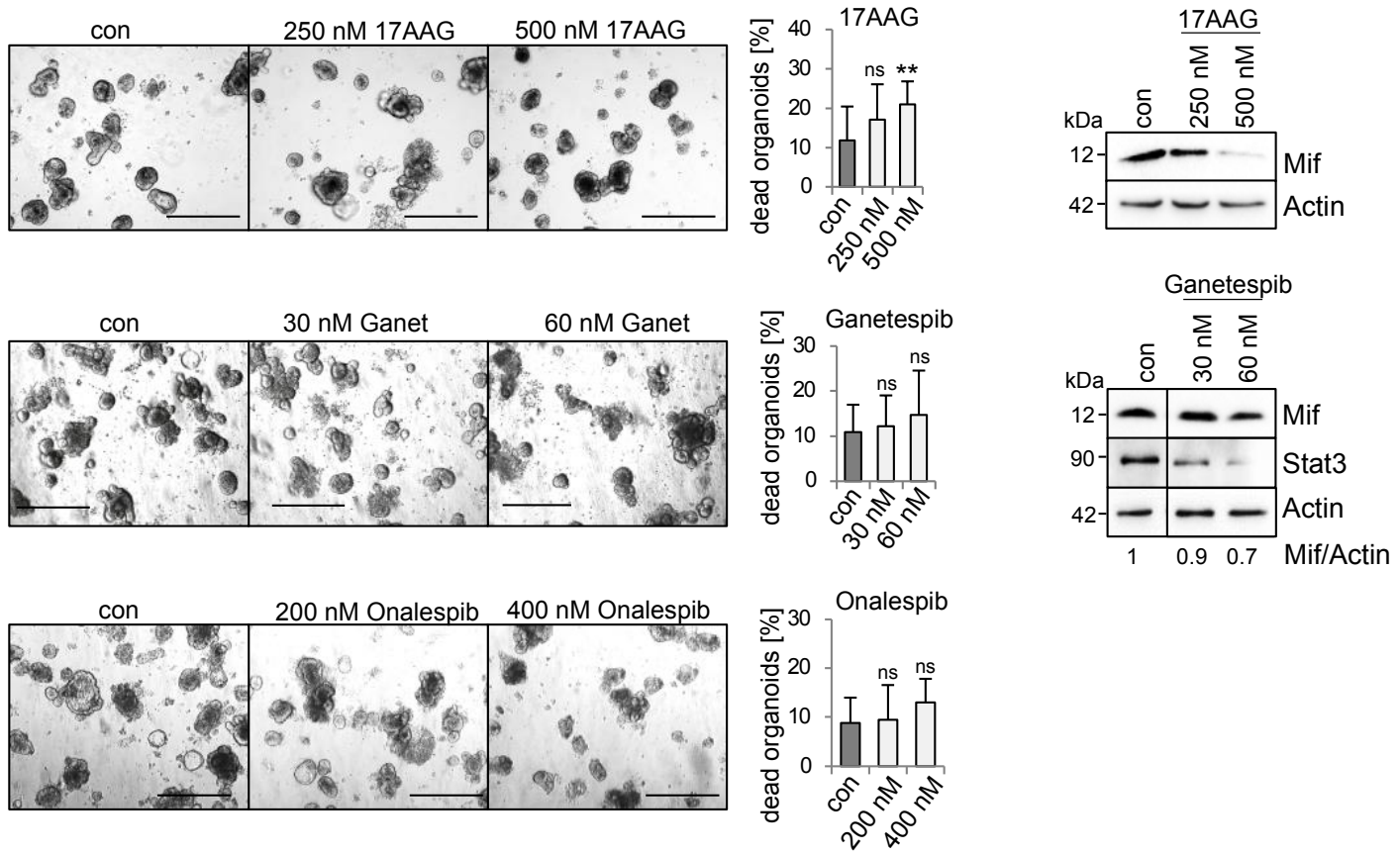

\section{Supp Figure 5}

Figure S5: Related to Figure 7. MIF is an actionable and selective therapeutic target by Hsp90 inhibition in colorectal cancer-derived organoids. (A) Representative images to evaluate growth and development of normal colonic organoids from two different mouse strains (129S1/SvImJ and C57BL/6). Images were taken at day of organoid preparation ( $d 0$ ) as well as two (d 2) and six (d 6) days after preparation ( $\mathrm{p} 0=$ passage 0 ). Additional image taken three ( $\mathrm{d} 3$ ) days after splitting ( $\mathrm{p} 1=$ =passage 1 ). Scale bars, $200 \mu \mathrm{m}$. (B) Representative images of murine normal small intestinal organoids after treatment with DMSO control ('con') and indicated concentrations of 17AAG, Ganetespib ('Ganet') or Onalespib for $21 \mathrm{hrs}$. Scale bars, $200 \mu \mathrm{m}$. Quantification reveal percentage of dead organoids relative to the total amount of organoids ( $\geq 9$ images per condition from $\geq 5$ gel domes). Mean \pm SD from different images. $p$ values in relation to control ('con'). ${ }^{*} p \leq 0.05 ;{ }^{* * *} p \leq 0.001$. (C) Hsp90 inhibitor treatment as in (B) of matched pairs. Immunoblot analysis to evaluate Mif degradation. Well known Hsp90-stabilized protein Stat3 used as positive control for the treatment. Actin, loading control. Mif expression ratios (Mif/Actin) were calculated by densitometry, normalized to the loading control, vehicle control. 
Klemke et al. Cell Death and Disease (2021)

Supplemental material

Supplemental Table 1: Primers

\begin{tabular}{|c|c|c|c|}
\hline Gene & Origin & Forward & Reverse \\
\hline \multicolumn{4}{|c|}{ Quantitative RT-PCR } \\
\hline $\begin{array}{l}R P L P 0 \\
(36 B 4)\end{array}$ & Human & 5'-GATTGGCTACCCAACTGTTG & 5'-CAGGGGCAGCAGCCACAAA \\
\hline HPRT1 & Human & 5'-CCTGGCGTCGTGATTAGTGAT & 5'-GGGCTACAATGTGATGGCCT \\
\hline MIF_1 & Human & 5'-AGCAGCTGGCGCAGGCCAC & 5'-CTCGCTGGAGCCGCCGAAGG \\
\hline MIF_ 2 & Human & 5'-GCAGCTGGCGCAGGCCAC & 5'-GGAGCCGCCGAAGGCCATGA \\
\hline CD74 & Human & 5'-GGCAACATGACAGAGGACCA & 5'-AGTGACTCTTTCGGTGGAGC \\
\hline $\begin{array}{l}\text { CXCL8 } \\
\text { (IL8)_1 }\end{array}$ & Human & 5'-GCTCTGTGTGAAGGTGCAGTT & 5'-AATTTCTGTGTTGGCGCAGT \\
\hline $\begin{array}{l}\text { CXCL8 } \\
\text { (IL8)_2 }\end{array}$ & Human & 5'-ACACTGCGCCAACACAGAAA & 5'-TTGCTTGAAGTTTCACTGGCAT \\
\hline VEGFA & Human & 5'-CTCCACCATGCCAAGTGGT & 5'-GTCCACCAGGGTCTCGATTG \\
\hline $\begin{array}{l}\text { Rplp0 } \\
(36 B 4)\end{array}$ & Mouse & 5'-GCAGATCGGGTACCCAACTGTT & 5'-CAGCAGCCGCAAATGCAGATG \\
\hline Mif_1 & Mouse & 5'-TCCGTGCCAGAGGGGTTTCTGT & 5'ACGTTGGCAGCGTTCATGTCG \\
\hline Mif 2 & Mouse & 5'-CTCCGTGCCAGAGGGGTTTCT & 5'-GCACCACCGATCTTGCCGATG \\
\hline $\operatorname{Arg1}$ & Mouse & 5'-GAGCATGAGCTCCAAGCCAA & 5'-TCTCTCACGTCATACTCTGTTTCT \\
\hline$B a x$ & Mouse & 5'-GCTGATGGCAACTTCAACTGG & 5'-TGATCAGCTCGGGCACTTTAG \\
\hline $\mathrm{Bcl} 2$ & Mouse & 5'-GACTGAGTACCTGAACCGGC & 5'-AGTTCCACAAAGGCATCCCAG \\
\hline$B c / 2 / 1$ & Mouse & $\begin{array}{l}\text { 5'- } \\
\text { TCGCCGGAGATAGATTTGAATAACC }\end{array}$ & 5'-TGGGCTCAACCAGTCCATTG \\
\hline $\mathrm{Ccl} 2$ & Mouse & 5'-GTCCCTGTCATGCTTCTGGG & 5'-GAGTAGCAGCAGGTGAGTGG \\
\hline $\mathrm{Ccl} 5$ & Mouse & 5'-TCACCATATGGCTCGGACA & 5'-TTCTCTGGGTTGGCACACAC \\
\hline Ccnd1 & Mouse & 5'-GGAGCTGCTGCAAATGGAAC & 5'-CAGTCCGGGTCACACTTGA \\
\hline $\begin{array}{l}\text { Cdkn1a } \\
\text { (p21) }\end{array}$ & Mouse & 5'-GTGGCCTTGTCGCTGTCTT & 5'-GCGCTTGGAGTGATAGAAATCTG \\
\hline Clec7a_1 & Mouse & 5'-AGGAAG & $\begin{array}{l}\text { 5'- } \\
\text { TACCACAAAGCACAGGATTCCTAAA }\end{array}$ \\
\hline Clec7a_2 & Mouse & 5'-TGGGTGCCCTAGGAGGTTT & 5'-AACCATGGCCCTTCACTCTG \\
\hline $\begin{array}{l}\mathrm{Cxcl1} \\
(\mathrm{Kc})\end{array}$ & Mouse & 5'-GCTGGGATTCACCTCAAGAA & 5'-CTTGGGGACACCTTTTAGCA \\
\hline $\mathrm{Cxcl} 2$ & Mouse & 5'-CTCTCAAGGGCGGTCAAAAAG & 5'-TTGGTTCTTCCGTTGAGGGAC \\
\hline Gadd45a & Mouse & 5'-AAGCTGCTCAACGTAGACCCC & 5'-ATCCATGTAGCGACTTTCCCG \\
\hline $1 / 1 b$ & Mouse & 5'AGCTTCCTTGTGCAAGTGTCT & 5'-GACAGCCCAGGTCAAAGGTT \\
\hline$M c / 1$ & Mouse & 5'-TAAGGACGAAACGGGACTGG & 5'AGTTTGGTGGCTGGAGCTTTA \\
\hline$M d m 2$ & Mouse & 5'-GGTCCCTGTCCTTTGATCCG & 5'-GCTCACTTACGCCATCGTCA \\
\hline Mmp9 & Mouse & 5'-TCTGTCCAGACCAAGGGTACA & 5'-GCCTTGGGTCAGGCTTAGAG \\
\hline Mrc1 & Mouse & 5'-TTGGTGGCAATTCACGAGAG & 5'-GGGAAGGGTCAGTCTGTGTTTG \\
\hline Nos2 & Mouse & 5'-CCCTCCTGATCTTGTGTTGGA & 5'-CAACCCGAGCTCCTGGAAC \\
\hline Ptgs2 & Mouse & 5'-TCCTGACCCACTTCAAGGGA & 5'-СТССТТАТТТСССТТСАСАСССА \\
\hline Tnfa & Mouse & 5'-AGGGATGAGAAGTTCCCAAATG & 5'-TGTGAGGGTCTGGGCCATA \\
\hline Vegfa 1 & Mouse & 5'-ACTGGACCCTGGCTTTACTG & 5'-GATCCGCATGATCTGCATGG \\
\hline Vegfa 2 & Mouse & 5'-CTGGACCCTGGCTTTACTGC & 5'-TGAACTTGATCACTTCATGGGACT \\
\hline \multicolumn{4}{|c|}{ Genotyping } \\
\hline Mif $A 1$ & Mouse & 5'AGGTTAGTCACTCTACTGGCC & \\
\hline Mif B1 & Mouse & 5'-TCTCACTGTTCTGGTGTGAGG & \\
\hline Mif C1 & Mouse & 5'-GGCTCCTGGTCTCAGTCAGG & \\
\hline Vil Cre & Mouse & 5'-CGCGAACATCTTCAGGTTCT & \\
\hline Vil Cre & Mouse & 5'-CAAGCCTGGCTCGACGGCC & \\
\hline
\end{tabular}


Klemke et al. Cell Death and Disease (2021)

Supplemental material

Supplemental Table 2: Reagents and Resources

\begin{tabular}{|c|c|c|}
\hline REAGENT or RESOURCE & SOURCE & IDENTIFIER (Cat\#) \\
\hline \multicolumn{3}{|l|}{ Antibodies } \\
\hline Rabbit polyclonal anti-Akt & Cell Signaling & 9272, RRID:AB_329827 \\
\hline $\begin{array}{l}\text { Rabbit monoclonal anti-phospho-Akt } \\
\text { (Ser473) [D9E] }\end{array}$ & Cell Signaling & 4060, RRID:AB_2315049 \\
\hline Mouse polyclonal anti-beta-Actin & Abcam & ab6276, RRID:AB_2223210 \\
\hline Rabbit polyclonal anti-beta-Actin & Abcam & ab8227, RRID:AB 2305186 \\
\hline Rabbit monoclonal anti-CD3 [SP7] & Abcam & ab16669, RRID:AB_443425 \\
\hline Rat monoclonal anti-CD31 [SZ31] & Dianova & DIA-310, RRID:AB_2631039 \\
\hline Rat monoclonal anti-CD68 [FA-11] & Abcam & ab53444, RRID:AB 869007 \\
\hline Rat monoclonal anti-CD68 [FA-11] & eBioscience $^{\mathrm{TM}}$ & 14-0681-82, RRID:AB_2572857 \\
\hline Rabbit polyclonal anti-CD74 & Sigma-Aldrich & HPA010592, RRID:AB_1078482 \\
\hline $\begin{array}{l}\text { Rabbit monoclonal anti-Cleaved Caspase-3 } \\
\text { [5A1E] }\end{array}$ & Cell Signaling & 9664, RRID:AB_2070042 \\
\hline Rabbit polyclonal anti-FoxP3 & Abcam & ab54501, RRID:AB_880110 \\
\hline $\begin{array}{l}\text { Rabbit monoclonal anti-Histone H2A.X, } \\
\text { phospho (Ser139) [20E3] }\end{array}$ & Cell signaling & 9718, RRID:AB_2118009 \\
\hline Mouse monoclonal HSC70 [B-6] & Santa Cruz & sc-7298, RRID:AB 627761 \\
\hline Rabbit polyclonal anti-Ki67 & Abcam & ab15580, RRID:AB_443209 \\
\hline Rabbit polyclonal anti-p38 MAPK & Cell Signaling & 9212, RRID:AB_330713 \\
\hline $\begin{array}{l}\text { Rabbit monoclonal anti-phospho-p38 MAPK } \\
\text { (Thr180/Tyr182) [3D7] }\end{array}$ & Cell Signaling & 9215, RRID:AB_331762 \\
\hline Rabbit polyclonal anti-ERK & Santa Cruz & Sc-94, RRID:AB_2140110 \\
\hline $\begin{array}{l}\text { Rabbit monoclonal anti-phospho-p44/42 } \\
\text { MAPK (Erk1/2) (Thr202/Tyr204) [D13.14.4E] }\end{array}$ & Cell Signaling & 4370, RRID:AB_2315112 \\
\hline Rabbit polyclonal anti-MIF & Sigma-Aldrich & HPA003868, RRID:AB_1079290 \\
\hline Goat polyclonal anti-MPO & R\&D system & AF3667, RRID:AB_2250866 \\
\hline Rabbit polyclonal anti-PARP & Cell Signaling & 9542, RRID:AB_2160739 \\
\hline Rabbit polyclonal anti-STAT3 & Santa Cruz & sc-482, RRID:AB 632440 \\
\hline Mouse monoclonal anti-VEGF [C1] & Santa Cruz & sc-7269, RRID:AB_ 628430 \\
\hline donkey anti-rat IgG $(\mathrm{H}+\mathrm{L})$ Alexa Fluor 594 & Invitrogen & A-21209, RRID:AB_2535795 \\
\hline ExtrAvidin $®$-Peroxidase & Sigma-Aldrich & E2886, RRID:AB_2620165 \\
\hline biotinylated goat/sheep antibody & GE Healthcare & RPN1025-2ML, RRID:AB_1082105 \\
\hline ImmPRESS TM Reagent Anti-Rabbit IgG & Vector Laboratories & MP-7401, RRID:AB_2336529 \\
\hline $\begin{array}{l}\text { ImmPRESS }{ }^{\mathrm{TM}} \text { Reagent Anti-Rat IgG, mouse } \\
\text { adsorbed }\end{array}$ & Vector Laboratories & MP-7444, RRID:AB_2336530 \\
\hline goat anti-rabbit IgG-HRP & Santa Cruz & SC-2004, RRID:AB_631746 \\
\hline goat anti-mouse IgG-HRP & Santa Cruz & sc-2005, RRID:AB_631736 \\
\hline \multicolumn{3}{|l|}{ Bacterial strains } \\
\hline Bacteria: ElectroMAX DH10B cells & $\begin{array}{l}\text { Invitrogen/Thermo } \\
\text { Fisher Scientific }\end{array}$ & $18290-015$ \\
\hline \multicolumn{3}{|c|}{ Chemicals, Peptides and Recombinant Proteins } \\
\hline $0.9 \%$ sodium chloride & B. Braun & 2001675 \\
\hline 17AAG & Provided by $\mathrm{NCl}$ & $\mathrm{N} / \mathrm{A}$ \\
\hline $\begin{array}{l}\text { 3,3'-Diaminobenzidine tetrahydrochloride } \\
\text { (DAB) }\end{array}$ & Roth & CN75.2 \\
\hline Ammonium sulfate $\left(\mathrm{NH}_{4}\right)_{2} \mathrm{SO}_{4}$ & Roth & 9218.1 \\
\hline AOM (Azoxymethane) & Sigma-Aldrich & A5486 \\
\hline BCA protein assay & Pierce & 23227 \\
\hline Clarity Max ${ }^{\mathrm{TM}}$ Western ECL Substrate & BioRad & 1705062 \\
\hline cOmplete ${ }^{\mathrm{TM}}$ mini protease inhibitor cocktail & Roche & 11836170001 \\
\hline Cycloheximide & Sigma-Aldrich & C7698 \\
\hline DAPI & Sigma-Aldrich & D9542 \\
\hline dATP & Primetech & 1202.4 \\
\hline dCTP & Primetech & 1203.4 \\
\hline
\end{tabular}


Klemke et al. Cell Death and Disease (2021)

Supplemental material

\begin{tabular}{|c|c|c|}
\hline Dextrose & Sigma-Aldrich & D9434 \\
\hline dGTP & Primetech & 1204.4 \\
\hline DirectPCR lysis Reagent & Peqlab & $31-101-T$ \\
\hline DSS (Dextran sodium sulfate) & MP Biomedicals & 160110 \\
\hline dTTP & Primetech & 1205.4 \\
\hline EDTA & Roth & 8040.1 \\
\hline Eosin G & Roth & 7089.1 \\
\hline Fluorescent Mounting Medium & DakoCytomation & S302380-2 \\
\hline Ganetespib & $\begin{array}{l}\text { Provided by Synta } \\
\text { Pharmaceuticals }\end{array}$ & N/A \\
\hline Onalespib (AT13387) & Selleckem & S1163 \\
\hline HRP substrate & Millipore/Merck & WBKLS0500 \\
\hline Imidazole & Roth & 3899.2 \\
\hline Isoflurane CP & CP-Pharma & 1214 \\
\hline Kolliphor $^{\circledR}$ RH 40 & Merck & 07076 \\
\hline Lipofectamine $^{\mathrm{TM}} 3000$ Transfection Reagent & Invitrogen & L3000015 \\
\hline M-MuLV Reverse Transcriptase & $\begin{array}{l}\text { New England } \\
\text { Biolabs }\end{array}$ & M0253S \\
\hline Magnesium chloride $\left(\mathrm{MgCl}_{2}\right)$ & Sigma-Aldrich & M1028 \\
\hline Mayer's Hemalum solution & Merck & 109249 \\
\hline Milk powder & Roth & T145.4 \\
\hline $\mathrm{NaCl}$ & Roth & 3957.2 \\
\hline Sodium Fluoride & Applichem & A0401 \\
\hline nitrocellulose membranes & Amersham & GE10600001 \\
\hline OneTaq® Quick-Load® 2X Master Mix & $\begin{array}{l}\text { New England } \\
\text { Biolabs }\end{array}$ & M0486L \\
\hline Phusion® High-Fidelity DNA Polymerase & $\begin{array}{l}\text { ThermoFisher } \\
\text { Scentific }\end{array}$ & F530 \\
\hline $\begin{array}{l}\text { Recombinant human Macrophage migration } \\
\text { inhibitory factor }\end{array}$ & Immunotools & 11344263 \\
\hline Roti $®$ Histokitt II & Roth & T160.1 \\
\hline SDS & Roth & CN30.3 \\
\hline Sodium deoxycholate & Sigma-Aldrich & 30970 \\
\hline Sodium orthovanadate & Sigma-Aldrich & S6508 \\
\hline SYBR Green & Invitrogen & S7567 \\
\hline Tamoxifen (TAM) & Sigma-Aldrich & T5648 \\
\hline Taq-polymerase & Primetech & 1800.4 \\
\hline Trehalose & Roth & 5151.3 \\
\hline Tris-HCl & Roth & 4855.3 \\
\hline Triton $\mathrm{X}-100$ & AppliChem & A1388 \\
\hline TRIzol $^{\mathrm{TM}}$ Reagent & Invitrogen & 15596026 \\
\hline TUNEL enzyme solution & Sigma-Aldrich & 11767305001 \\
\hline TUNEL Label mix & Sigma-Aldrich & 11767291910 \\
\hline Tween-20 & AppliChem & A4974 \\
\hline \multicolumn{3}{|l|}{ Reagents for Cell culture } \\
\hline A83-01 & Sigma-Aldrich & SML0788 \\
\hline Advanced DMEM/F12 medium & Gibco & 12634010 \\
\hline B-27 & Gibco & 17504044 \\
\hline Cell Recovery solution & Corning & 11543560 \\
\hline CHIR 99021 & Axon Medchem & 1386 \\
\hline Collagenase type I, powder & Gibco & 17018029 \\
\hline DMEM & Gibco & 61965059 \\
\hline FBS & Merck & S0615 \\
\hline Geneticin (G418) & InvivoGen & ant-gn-1 \\
\hline GlutaMAX $^{\mathrm{TM}}$ & Gibco & 35050061 \\
\hline HEPES & Gibco & 15630080 \\
\hline L-Glutamine & Gibco & 25030123 \\
\hline
\end{tabular}


Klemke et al. Cell Death and Disease (2021)

Supplemental material

\begin{tabular}{|c|c|c|}
\hline Matrigel & Corning & 354230 \\
\hline McCoy's 5A modified medium & Gibco & 16600082 \\
\hline Mycoplasma Detection Kit & Lonza & LT07-318 \\
\hline $\mathrm{N}-2$ & Gibco & 17502048 \\
\hline N-Acetyl-L-Cysteine & Sigma-Aldrich & A9165 \\
\hline Nicotinamide & Sigma-Aldrich & N3376 \\
\hline Penicillin-Streptomycin & Gibco & 15140122 \\
\hline rmEGF & ImmunoTools & 12343406 \\
\hline ROCK inhibitor (Y-27632) & Sigma-Aldrich & SCM075 \\
\hline RPMI 1640 & Gibco & 42401042 \\
\hline Sodium Pyruvate & Gibco & 11360039 \\
\hline Zeocin & InvivoGen & ant-zn-05 \\
\hline \multicolumn{3}{|l|}{ Experimental models: Cell lines } \\
\hline HEK293T Wnt3a & $\begin{array}{l}\text { Robyn Laura } \\
\text { Kosinsky }\end{array}$ & \\
\hline HEK293T Noggin & Farin et. al, 2012 & PMID: 22922422 \\
\hline HEK293T R-spondin I & Farin et. al, 2012 & PMID: 22922422 \\
\hline HCT116 & ATCC & ATCC $^{\circledR}$ CCL-247 ${ }^{\mathrm{TM}}$ \\
\hline DLD-1 & DSMZ & ACC 278 \\
\hline \multicolumn{3}{|l|}{ Experimental models: Mouse strains } \\
\hline Mif knock-out (129S1/SvImJ) & $\begin{array}{l}\text { Fingerle-Rowson et } \\
\text { al., } 2003\end{array}$ & PMID: 12878730 \\
\hline $\mathrm{Mif}^{f / / f 1}(\mathrm{C} 57 \mathrm{BL} / 6 \mathrm{~N})$ & $\begin{array}{l}\text { Fingerle-Rowson et } \\
\text { al., } 2003 \text {, Brocks et } \\
\text { al., } 2017\end{array}$ & PMID: 12878730, PMID: 27825106 \\
\hline Mouse: villin:CreERT2 & $\mathrm{N} / \mathrm{A}$ & RRID:IMSR_JAX:020282 \\
\hline C57BL/6NCrl & Charles River & RRID:IMSR CRL:27 \\
\hline \multicolumn{3}{|l|}{ Oligonucleotides and Recombinant DNA } \\
\hline Primers for qPCR and genotyping & this paper & Table 1 \\
\hline $\begin{array}{l}\text { siRNA Silencer }{ }^{\mathrm{TM}} \text { Select Negative Control } \\
\text { No. } 2 \text { siRNA ( } \mathrm{src} 2)\end{array}$ & Invitrogen & 4390847 \\
\hline siRNA MIF Silencer ${ }^{\mathrm{TM}}$ Select & $\begin{array}{l}\text { ThermoFisher } \\
\text { Scientific }\end{array}$ & 4390824, ID s8780 \\
\hline siRNA MIF Silencer ${ }^{\mathrm{TM}}$ & $\begin{array}{l}\text { ThermoFisher } \\
\text { Scientific }\end{array}$ & AM51331, ID 11396 \\
\hline siRNA hCD74 Silencer ${ }^{\mathrm{TM}}$ Select & $\begin{array}{l}\text { ThermoFisher } \\
\text { Scientific }\end{array}$ & 4392420, ID s2715 \\
\hline siRNA hCD74 Silencer ${ }^{\mathrm{TM}}$ Select & $\begin{array}{l}\text { ThermoFisher } \\
\text { Scientific }\end{array}$ & 4392420, ID s225179 \\
\hline pcDNA3 GFP & Addgene & 74165 \\
\hline pcDNA3.1/V5-His-TOPO vector & Shi et al., 2006 & PMID: 17045821 \\
\hline pcDNA3.1-CD74 & Shi et al., 2006 & PMID: 17045821 \\
\hline \multicolumn{3}{|l|}{ Software and Algorithms } \\
\hline Adobe Photoshop Software & Adobe & $\begin{array}{l}\text { https://www.adobe.com/de/creative } \\
\text { cloud/plans.html }\end{array}$ \\
\hline GraphPad Prism & GraphPad & https://www.graphpad.com \\
\hline ImageJ software & Open source & https://imagej.net/Welcome \\
\hline Image Lab ${ }^{\text {TM }}$ Software & Biorad & $\begin{array}{l}\text { http://www.bio-rad.com/de- } \\
\text { de/product/image-lab-software }\end{array}$ \\
\hline ZEN & Zeiss & $\begin{array}{l}\text { https://www.zeiss.de/mikroskopie/pr } \\
\text { odukte/mikroskopsoftware/zen.html }\end{array}$ \\
\hline
\end{tabular}




\title{
3.2 MANUSCRIPT: MUTP53 IN PDAC
}

\section{THE GAIN-OF-FUNCTION P53 R248W MUTANT PROMOTES MIGRATION BY STAT3 DEREGULATION IN HUMAN PANCREATIC CANCER CELLS}

\author{
Luisa Klemke $^{1}$, Clara F Fehlau ${ }^{1}$, Nadine Winkler ${ }^{1}$, Felicia Toboll ${ }^{1}$, Shiv K Singh ${ }^{2}$, Ute \\ $\mathrm{M} \mathrm{Moll}^{3}$ and Ramona Schulz-Heddergott ${ }^{1^{*}}$ \\ ${ }^{1}$ Institute of Molecular Oncology, University Medical Center Göttingen, Germany. \\ 2 Department of Gastroenterology, Gastrointestinal Oncology, and Endocrinology, University Medical Center \\ Göttingen, Germany. \\ 3 Department of Pathology, Stony Brook University, Stony Brook NY 11794, USA \\ *Corresponding author: ramona.schulz@zentr.uni-goettingen.de
}

\section{RUNNING TITLE}

Missense mutant p53 function in PDAC

Submitted to: Frontiers in Oncology

Status: Provisionally accepted (April 2021)

DOI: $10.3389 /$ fonc.2021.642603

\section{Own contribution:}

Conducted experiments and data analysis for: Figure 1A, Figures 2A, B, Figures 3C, D, Figures 4A, E-G, Figure 5A-D, Figures S1, Supp. Fig. 2B. Data analysis for: Figure 1B, Figures 4B-D, $\mathrm{H}$, as well as preparation of additional supplemental material. Involved in the conceptualization, methodology, acquisition, analysis and interpretation of data, figure arrangement, writing and revising the manuscript.

${ }^{* *}$ Figures and corresponding figure legends are located at the end of the manuscript 
Klemke et al., manuscript provisionally accepted by Frontiers in Oncology (April 2021)

\section{The gain-of-function p53 R248W mutant promotes migration by STAT3 deregulation in human pancreatic cancer cells}

Luisa Klemke ${ }^{1}$, Clara Friederike Fehlau ${ }^{1}$, Nadine Winkler ${ }^{1}$, Felicia Toboll ${ }^{1}$, Shiv K Singh ${ }^{2}$, Ute M Moll $^{3}$ and Ramona Schulz-Heddergott ${ }^{1^{*}}$

${ }^{1}$ Institute of Molecular Oncology, University Medical Center Göttingen, Germany

2 Department of Gastroenterology, Gastrointestinal Oncology, and Endocrinology, University Medical Center Göttingen, Germany

${ }^{3}$ Department of Pathology, Stony Brook University, Stony Brook NY 11794, USA

* Corresponding author: ramona.schulz@zentr.uni-goettingen.de

\section{RUNNING TITLE}

Missense mutant p53 function in PDAC

\section{CORRESPONDING AUTHOR}

Dr. Ramona Schulz-Heddergott

Institute of Molecular Oncology, Universitätsmedizin Göttingen (UMG)

Justus-von-Liebig-Weg 11, 37077 Göttingen, Germany

E-mail: ramona.schulz@zentr.uni-goettingen.de

Phone: +49 5513960780

Fax: +495513960747

\section{GRANT SUPPORT}

R. S.-H. and L. K. are supported by the Deutsche Forschungsgemeinschaft (DFG, SCHUH-3160/3-1). R.S.H. and S.K.S are supported by the Clinical Research Unit KFO5002 (DFG). U. M. M. is supported by the NIH National Cancer Institute (2R01CA176647).

\section{AUTHOR CONTRIBUTIONS}

Conceptualization R. S.-H., L. K.; Methodology R. S.-H., L. K., C. F. F., S. K. S.; Experimentation L. K., C. F. F, N. W., F. T.; Writing Original Draft L. K., U. M. M.; Writing Review \& Editing: all authors; Funding Acquisition U. M. M, R. S.-H.; Supervision R. S.-H.

\section{CONFLICT OF INTEREST}

All authors declare to have no conflict of interests. 


\section{ABSTRACT}

Missense p53 mutations (mutp53) occur in approx. 70\% of pancreatic ductal adenocarcinomas (PDAC). Typically, mutp53 proteins are aberrantly stabilized by Hsp90/Hsp70/Hsp40 chaperone complexes. Notably, stabilization is a precondition for specific mutp53 alleles to acquire powerful neomorphic oncogenic gain-of-functions (GOFs) that promote tumor progression in solid cancers mainly by increasing invasion and metastasis. In colorectal cancer (CRC) we recently established that the common hotspot mutants mutp53 ${ }^{\mathrm{R} 248 \mathrm{Q}}$ and mutp53 $3^{\mathrm{R} 248 \mathrm{~W}}$ exert GOF activities by constitutively binding to and hyperactivating STAT3. This results in increased proliferation and invasion in an autochthonous CRC mouse model and correlates with poor survival in patients.

Comparing a panel of p53 missense mutations in a series of homozygous human PDAC cell lines, we show here that similar to CRC the mutp53 ${ }^{\mathrm{R} 248 \mathrm{~W}}$ protein again undergoes a strong Hsp90mediated stabilization and selectively promotes migration. Highly stabilized mutp53 is degradable by the Hsp90 inhibitors Onalespib and Ganetespib, and correlates with growth suppression, possibly suggesting therapeutic vulnerabilities to target GOF mutp53 proteins in PDAC.

In response to mutp53 depletion only mutp53 ${ }^{\text {R248W }}$ harboring PDAC cells show STAT3 dephosphorylation and reduced migration, again suggesting an allele-specific GOF in this cancer entity, similar to CRC. Moreover, mutp53 ${ }^{\mathrm{R} 248 \mathrm{~W}}$ also exhibits the strongest constitutive complex formation with phosphorylated STAT3. The selective mutp53 ${ }^{\mathrm{R} 248 \mathrm{~W}} \mathrm{GOF}$ signals through enhancing the STAT3 axis, which was confirmed since targeting STAT3 by knockdown or pharmacological inhibition phenocopied mutp53 depletion and reduced cell viability and migration preferentially in mutp53 ${ }^{\mathrm{R} 248 \mathrm{~W}}$-containing PDAC cells. Our results confirm that mutp53 GOF activities are allele specific and can span across tumor entities. 


\section{INTRODUCTION}

Already in the early 1990s the tumor suppressor p53 was coined as 'guardian of the genome' (Lane, 1992; Zilfou and Lowe, 2009) and it was known that mutation of the TP53 gene (tumor protein p53, HGNC:11998) is an essential step in human tumor development (Hollstein et al., 1991; Lane, 1992). Ever since scientists have tried to understand the influence of the TP53 status within the mutational landscape in different cancer entities and to investigate the role of different variants in tumorigenic pathways. It became evident that some p53 mutant protein variants do not only abrogates tumor suppressor functions (loss-of-function, LOF), but also gain new tumorigenic functions (gain-of-functions; GOFs). Given that approx. $70 \%$ are missense mutations leading to amino acid substitutions mostly in the DNA binding domain, some alleles are selected and occur at a high frequency, termed hotspots. Most hotspot mutants gain neomorphic tumorigenic functions, particularly in invasion and metastasis of solid tumors (Brosh and Rotter, 2009; Oren and Rotter, 2010; Goh et al., 2011; Kim and Lozano, 2018; Sabapathy and Lane, 2018; Mantovani et al., 2019). A key prerequisite for GOFs of some missense p53 mutants (termed here 'mutp53') is protein stabilization through the Hsp90/Hsp70/Hsp40 (heat shock protein 90/70/40) chaperone machinery, resulting in protection from MDM2 (mouse double minute 2) and other E3 ligases and thus proteasomal degradation (Walerych et al., 2004; Walerych et al., 2009; Wiech et al., 2012; Parrales et al., 2016; Schulz-Heddergott and Moll, 2018; Wawrzynow et al., 2018; Mantovani et al., 2019).

Due to the heterogeneity of TP53 point mutations, whose phenotype in addition are highly dependent on the cellular context, different missense mutants exert different cellular responses (Freed-Pastor and Prives, 2012; Lee et al., 2012; Gencel-Augusto and Lozano, 2020). Thus, it is important to consider the context- and allele-dependent specificity of different mutp53 proteins (Lee et al., 2012; Walerych et al., 2015; Ubby et al., 2019; Kadosh et al., 2020). To investigate the mutp53 specificity, different groups have dissected the impact of various mutp53 GOF alleles on tumorigenesis using autochthonous mouse models (Hanel et al., 2013; Alexandrova et al., 2015; Kim et al., 2015; Schulz-Heddergott et al., 2018; Zhang et al., 2018) or clinical correlation studies (Said et al., 2013; Xu et al., 2014; Alexandrova et al., 2017; Schulz-Heddergott et al., 2018). Recent results from our group highlight the GOF hotspot mutp53 ${ }^{R 248 Q / W}$ specificity in mouse and human colorectal cancer (CRC). mutp53 ${ }^{\mathrm{R} 248 \mathrm{Q} / \mathrm{W}}$ binds to and deregulates phosphorylated STAT3 (signal transducer and activator of transcription 3) by protecting it from SHP2 phosphatase (PTPN11, protein tyrosine phosphatase non-receptor type 11), its major negative regulator. Thus, 
depletion of mutp53 ${ }^{\mathrm{R} 248 \mathrm{Q} / \mathrm{W}}$ inhibits STAT3 signaling and causes suppression of tumor invasion and proliferation (Schulz-Heddergott et al., 2018). The p53 R248 hotspot is the single most common variant in all TP53-altered tumor types occurring in 9\%, of cases which translates to about 66,000 newly diagnosed cancer patients in the US per year harboring R248 variants. Of R248 substitutions, over $90 \%$ are either $Q$ or W, with similar frequencies (The Cancer Genome Atlas Program - National Cancer Institute).

Here we asked whether mutp53 ${ }^{\mathrm{R} 248 \mathrm{~W}}$ also exhibits tumor-promoting functions affecting migration in pancreatic ductal adenocarcinoma (PDAC). Note, the TP53 ${ }^{\mathrm{R} 248 \mathrm{Q}}$ allele is not available in established PDAC lines. PDAC is currently the fourth leading cause of cancer death worldwide with a rapidly ascending trajectory, and incidence is predicted to increase even further in the future (Orth et al., 2019; Siegel et al., 2020). PDAC, which constitutes around 90\% of all pancreatic malignancies, is highly aggressive and chemoresistant and still has a dismal 5 year survival rate of only approx. 9\% (Grasso et al., 2017; Smigiel et al., 2018; Orth et al., 2019; American-Cancer-Society, 2020).

In approx. $70 \%$ of PDAC patients TP53 undergoes mainly missense mutations (www.cbioportal.org) as a late genetic event at the transition from high grade PanIN dysplasia to invasiveness during pancreatic cancer progression (Guo et al., 2016; Cicenas et al., 2017). Here we show in a panel of common human PDAC cell lines harboring different homozygous missense p53 mutants that mutp53 variants differ in their protein stability, with mutp53 ${ }^{\mathrm{R} 248 \mathrm{~W}}$ again accumulating the highest protein levels also in the pancreatic cell context. Importantly, comparing all PDAC lines only mutp53 $3^{\mathrm{R} 248 \mathrm{~W}}$ depletion strongly reduced migration capacity. In support, mutp53 ${ }^{\mathrm{R} 248 \mathrm{~W}}$ specifically showed the strongest binding to phosphorylated STAT3 under baseline and cytokine-stimulated conditions, forming a constitutive mutp53 ${ }^{R 248 \mathrm{~W}}$-pSTAT3 complex. Only mutp53 ${ }^{\mathrm{R} 248 \mathrm{~W}}$ depletion was able to reduce pSTAT3 levels. Consequently, targeting the tumorpromoting mutp53 ${ }^{\mathrm{R} 248 \mathrm{~W}}$-pSTAT3 complex by pSTAT3 depletion or pharmacological inhibition diminished cell viability and migration in mutp53 ${ }^{\mathrm{R} 248 \mathrm{~W}}$ expressing, but not in mutp53 ${ }^{\mathrm{R} 273 \mathrm{H}}$ or mutp53 ${ }^{\mathrm{R} 282 \mathrm{~W}}$ expressing PDAC cells. Our results support a GOF function of mutp53 ${ }^{\mathrm{R} 248 \mathrm{~W}}$ in pancreatic cancer cell lines, justifying future investigations in this tumor entity in vivo. 


\section{RESULTS}

\section{p53 missense mutants in human PDAC cell lines are stabilized via HSP90}

Since different p53 mutants have different conformations and thus different tumorigenic functions that additionally depend on specific cellular/oncogenic context, each allele and tumor type constellation should be considered separately (Bullock et al., 2000; Freed-Pastor and Prives, 2012; Kim and Lozano, 2018; Schulz-Heddergott and Moll, 2018). To investigate the allele specificity of mutated TP53 in pancreatic ductal adenocarcinoma (PDAC), we used homozygous human PDAC cell lines expressing different endogenous p53 hotspot and non-hotspot missense

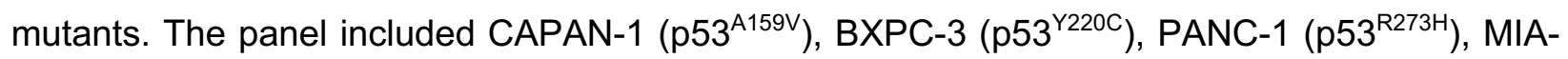
PACA-2 (p53 $\left.{ }^{\mathrm{R} 248 \mathrm{~W}}\right)$, PA-TU-8902 (p53 $\left.{ }^{\mathrm{C} 176 \mathrm{~S}}\right)$ and PA-TU-8988T (p53 $\left.{ }^{\mathrm{R} 282 \mathrm{~W}}\right)$. L3.6pl harbors a truncating LOF mutation and served as p53null control. Unfortunately, an established PDAC line with a mutated TP53 ${ }^{\mathrm{R} 248 \mathrm{Q}}$ allele is not available. The absence of wildtype $\mathrm{p} 53$ function was verified in all cases (Figure S1).

Comparative immunoblot analysis identified the highest steady state protein levels in MIAPACA-2 cells expressing the R248W mutant (Figure 1A). The second highest levels were observed in C176S and R282W harboring PA-TU-8902 and PA-TU-8988T cells, respectively. The lowest level was seen in A159V expressing CAPAN-1 cells (Figure 1A). Cycloheximide chase experiments confirmed that the highest p53 steady state levels in cells harboring mutp53 ${ }^{\mathrm{R} 248 \mathrm{~W}}$, mutp53 ${ }^{\mathrm{C} 176 \mathrm{~S}}$ and mutp53 ${ }^{\mathrm{R} 282 \mathrm{~W}}$ were also the most stable proteins with the longest half-lives, while mutant p53 protein with the lowest level (A159V) had the shortest half-life (Figure 1B).

A key prerequisite for the gain-of-function (GOF) of some missense p53 mutants is protein stabilization through the Hsp90 chaperone machinery. Importantly, the clinically relevant Hsp90 inhibitors Ganetespib or Onalespib provide therapeutic selectivity towards tumor epithelial cells but not normal cells, making them attractive for anti-cancer therapies (Klemke et al., 2021). Furthermore, in other cellular contexts such as lymphoma (Alexandrova et al., 2015), treatment with the Hsp90 inhibitors Ganetespib or Onalespib also downregulated mutp53 protein levels in most PDAC cells, except BXPC-3 cells (Figure 2A), indicating that mutp53 proteins are mainly stabilized in this context by the Hsp90 chaperone machinery. In line, PANC-1, MIA-PACA-2, PATU-8902 and PA-TU-8988T cells showed diminished cell growth by about $40 \%$, while the other lines had less reduction (Figure 2B). These data reinforce that at least some mutp53 proteins in PDAC might also be targetable with Hsp90 inhibitors. 


\section{The $\mathrm{p} 53^{\mathrm{R} 248 \mathrm{~W}}$ mutant selectively promotes migration in PDAC cells}

We previously established that a main GOF activity of the mutp53 ${ }^{\mathrm{R} 248 \mathrm{~W}}$ and mutp $53^{\mathrm{R} 248 \mathrm{Q}}$ in colorectal cancer compared to p53 null is promotion of cell migration and invasion in tumors in vivo and in vitro (Schulz-Heddergott et al., 2018). To test whether this is also the case in PDAC we performed migration assays. Of note, transwell migration assays showed that only siRNAmediated depletion of mutp53 ${ }^{\mathrm{R} 248 \mathrm{~W}}$ decreased the migration capacity of MIA-PACA-2 cells, while depletion of other alleles failed to do so (Figures 3A-D). Interestingly, PA-TU-8988T and PA-TU8902 cells which also express high levels of stabilized mutp53 ${ }^{\mathrm{R} 282 \mathrm{~W}}$ or mutp53 ${ }^{\mathrm{C} 176 \mathrm{~S}}$ respectively (Figures 1A, B) did not show reduced migration after mutp53 depletion (Figure 3C) or were completely unable to migrate through the pores of the transwell membrane (Figure $3 \mathrm{E}$ ). This remained even after treatment of PA-TU-8902 cells with the cytokines Interleukin-6 (IL-6) and Oncostatin M (OSM) (Figure 3E), known to induce migration and proliferation in numerous cell types (Natesh et al., 2015; Razidlo et al., 2018; Che et al., 2019). This suggests that high mutp53 stabilization per se is a necessary but not sufficient precondition for acquiring a GOF on migration.

To confirm the effects seen in migration assays, 3 cell lines were further analyzed by wound healing scratch assays. Again, specifically MIA-PACA-2 cells bearing the R248W mutation showed the strongest reduction in wound closing capacity upon mutp53 depletion (Figures 3F$\mathrm{H})$.

\section{Mutp53 ${ }^{\mathrm{R248W}}$ selectively binds to phosphorylated STAT3 in PDAC cells}

In colorectal carcinoma an important mechanism of tumor invasion is mediated by mutp53 $3^{\mathrm{R} 248 \mathrm{Q} / \mathrm{W}_{\text {- }}}$ pSTAT3 signaling by forming a physical complex (Schulz-Heddergott et al., 2018). Reduced migration capacity of MIA-PACA-2 cells after mutp53 ${ }^{\mathrm{R} 248 \mathrm{~W}}$ depletion (Figures $3 \mathrm{~A}, \mathrm{~F}$ ) suggests a similar mechanism. Since the STAT3 pathway is also an important driver of PDAC tumorigenesis (Denley et al., 2013; Nagathihalli et al., 2015), we asked whether mutp53 ${ }^{\mathrm{R} 248 \mathrm{~W}}$-regulated migration is similarly mediated through STAT3 signaling. The PDAC panel showed high constitutive levels of phosphorylated STAT3 (pSTAT3) in 5 of the 7 cell lines (Figure 4A). Only 2 cell lines, PA-TU8902 and PA-TU-8988T, had very low levels of activated STAT3 (yet exhibited significant stabilization of mutp53). On the other hand, this immunoblot analysis which examines relative ratios of both proteins indicated that 4 lines with high pSTAT3 had very low or undetectable mutp53 levels. Importantly, MIA-PACA-2 cells, as the only cell line with dually high levels of both mutp53 and pSTAT3, seem to fulfill the best precondition to promote migration via this axis. 
Thus, co-immunoprecipitations (ColPs) were performed to test which of the various mutp53 proteins are able to bind STAT3. Indeed, R248W in MIA-PACA-2 cells showed the strongest binding to total STAT3 protein compared to BXPC-3 and PANC-1 cells, forming a constitutive endogenous signaling complex (Figure S2A). Since phosphorylation status is another important parameter for binding to STAT3, these cell lines with different mutp53 variants and stabilization levels were subjected to ColPs with an antibody specific for phosphorylated STAT3. Among these mutants analyzed, mutp53 $3^{\mathrm{R} 248 \mathrm{~W}}$ in MIA-PACA-2 cells again showed the strongest binding to pSTAT3 (Figure 4B). CAPAN-1 cells with low mutp53 level showed a minor binding to pSTAT3 (Figure 4C) such as BXPC-3 and PANC-1 cells (Figure 4B) (yet exhibited moderate levels of mutp53 compared to CAPAN-1). However, PA-TU-8988T cells with intermediate mutp53 levels (lower than in MIA-PACA-2 but higher than in PANC-1 or BXPC-3 cells) again showed a strong binding of mutp53 $3^{\mathrm{R} 282 \mathrm{~W}}$ to pSTAT3 (Figure 4D). This confirms a point made in colorectal carcinoma that the ability of mutp53 to bind to pSTAT3 correlates with the degree of its stabilization (Schulz-Heddergott et al., 2018).

To investigate if the mutp53-pSTAT3 complex can directly regulate the phosphorylation status of STAT3 as shown in CRC (Schulz-Heddergott et al., 2018), we depleted mutp53 in MIA-PACA-2, PA-TU-8988T, PANC-1, BXPC-3 and PA-TU-8902 cells (Figures 4E, S2B). In MIA-PACA-2 and PA-TU-8988T cells, both with a strong mutp53-pSTAT3 complex formation, only mutp53 ${ }^{\text {R248W }}$ regulated STAT3 activity in PDAC cells, as indicated by decreased STAT3 phosphorylation selectively in MIA-PACA-2 cells (Figure 4E). In all other cell lines tested, pSTAT3 level were not decreased after mutp53 depletion (Figures 4E, S2B). Why mutp53 binding to pSTAT3 failed to reduce STAT3 activity in PA-TU-8988T cells remains speculative but confirms the reduced migration capacity after mutp53 depletion exclusively in MIA-PACA-2 cells (Figures 3A-D). These data further underline the strong invasive GOF function of the mutp53 ${ }^{\mathrm{R} 248 \mathrm{Q} / \mathrm{W}}$ allele reaching across cancer entities.

Although most PDAC cell lines already exhibited high constitutive levels of pSTAT3 at baseline (Figure 4A), treatment with Interleukin-6 (Figure 4F) or Oncostatin M (Figure 4G) further stimulated the STAT3 pathway and induced additional increase in phosphorylated STAT3. Thus, to further evaluate whether the mutp53 binding capacity to pSTAT3 increases with higher pSTAT3 levels, MIA-PACA-2, as well as PANC-1 and BXPC-3 cells (both with a low binding capacity) were treated with IL-6, OSM or solvent control. Interestingly, even after this strong induction of pSTAT3, the $\mathrm{p} 53^{\mathrm{R} 248 \mathrm{~W}}$ mutant showed by far the strongest binding to pSTAT3, again emphasizing allele selectivity (Figure $4 \mathrm{H}$ ). These data suggest that it is not the level of pSTAT3 that predicts p53 binding in PDAC, but rather the nature of the mutp53 variant. In sum, mutp $53^{\mathrm{R} 248 \mathrm{~W}}$ shows a strong 
ability for complexing with pSTAT3 and regulation of migration, independent of the levels of phosphorylated STAT3.

\section{Mutp53 ${ }^{\mathrm{R} 248 \mathrm{~W}}$ selectively regulates STAT3 phosphorylation and activity in PDAC cells}

The above findings led us to hypothesize that mutp53 ${ }^{\mathrm{R} 248 \mathrm{~W}}$ binds to and deregulates pSTAT3 in PDAC cells by forming an oncogenic complex. Since mutp53 ${ }^{\mathrm{R} 248 \mathrm{~W}}$ depletion also selectively suppressed phosphorylation and thus activation of STAT3 (Figure 4E), we next asked whether the R248W mutant can be functionally linked to STAT3 dependency for migration in PDAC cells. To this end we determined migration capacity after STAT3 ablation. Indeed, depletion of STAT3 suppressed migration ability in mutp53 ${ }^{\mathrm{R} 248 \mathrm{~W}}$ expressing MIA-PACA-2 cells (Figure 5A) but not in mutp53 ${ }^{\mathrm{R} 273 \mathrm{H}}$ expressing PANC-1 cells (Figure 5B).

To confirm that phosphorylated STAT3 is critical for the oncogenic mechanism of the tumorpromoting mutp53 ${ }^{\mathrm{R} 248 \mathrm{~W}}$-pSTAT3 complex, we used the small-molecule STAT3 inhibitor Stattic. Stattic selectively inhibits activation of STAT3 through interference with dimerization and nuclear translocation (Schust et al., 2006). It has been shown that Stattic substantially reduces STAT3 phosphorylation in colorectal, liver and breast cancer cells (Schust et al., 2006; Lin et al., 2011; Spitzner et al., 2014) as well as in PDAC cells such as MIA-PACA-2 and PANC-1 (Cardoso et al., 2012; Lin et al., 2016; Nagathihalli et al., 2018). Importantly, among the panel of PDAC cells, R248W expressing MIA-PACA-2 cells were again the most susceptible to pSTAT3 inhibition by Stattic with the lowest $\mathrm{IC}_{50}$ value $(8 \mu \mathrm{M})$ in cell viability assays (Figure $5 \mathrm{C}$ ). Likewise, migration after Stattic treatment was strongly suppressed in MIA-PACA-2 cells (by $\sim 70 \%$ ), but lower suppressed in PANC-1 (by $\sim 15 \%$ ) or PA-TU-8988T cells (by $\sim 45 \%$ ) cells (Figure 5D).

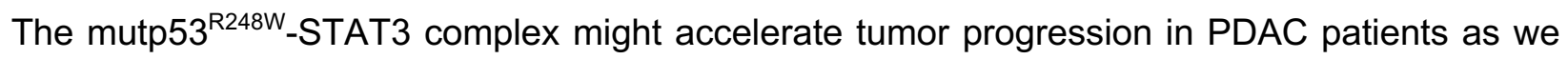
had previously seen in CRC patients (Schulz-Heddergott et al., 2018). Indeed, TCGA patient data support this notion since PDAC patients harboring $T P 53^{R 248 Q}$ or $T P 53^{R 248 W}$ mutations showed a trend for reduced survival compared to patients with loss-of-function NS+FS mutation (Figure 5E), supporting the mutp53 $3^{\mathrm{R} 248 \mathrm{~W}}$-pSTAT3 complex as a potentially attractive target in PDAC.

In conclusion, targeting the tumor-promoting mutp53 ${ }^{\mathrm{R} 248 \mathrm{~W}}$-pSTAT3 complex by STAT3 depletion or pharmacological inhibition diminished cell viability and migration in mutp53 ${ }^{\mathrm{R} 248 \mathrm{~W}}$ expressing, but not in mutp53 ${ }^{\mathrm{R} 273 \mathrm{H}}$ expressing, PDAC cells. 


\section{DISCUSSION}

The phenotype of p53 missense mutants is heterogenous and moreover depends on the cellular context (Freed-Pastor and Prives, 2012; Lee et al., 2012; Gencel-Augusto and Lozano, 2020). Here we analyze a panel of p53 missense mutants (mutp53) in a series of homozygous human PDAC cell lines and compare the impact of various mutants on protein properties and functions. We find that mutp53 $3^{\mathrm{R} 248 \mathrm{~W}}$ protein undergoes strong Hsp90-mediated stabilization and selectively promotes migration by engaging in the strong constitutive complex formation with phosphorylated STAT3 at baseline and upon cytokine stimulation. Our data in pancreatic cancer suggest a R248W allele-specific gain-of-function on migration via STAT3 deregulation. These data mirror our previous findings in colorectal cancer (Schulz-Heddergott et al., 2018) and further underline the necessity to investigate p53 missense mutants in a context and allele-dependent manner (Lee et al., 2012; Walerych et al., 2015; Ubby et al., 2019).

Interestingly, PA-TU-8902 cells expressing intermediate stabilized mutp53 ${ }^{\mathrm{C} 176 \mathrm{~S}}$ showed strong STAT3 pathway stimulation by OSM or IL-6 (Figures 4F, G) but did not migrate at all through the transwell assay (Figure 3E), indicating that STAT3 fails to impact migration in these cells. Furthermore, PA-TU-8988T cells harboring intermediate levels of mutp53 ${ }^{\mathrm{R} 282 \mathrm{~W}}$ showed a strong binding to pSTAT3 but failed to regulate pSTAT3 level (indicating STAT3 activity) (Figure 4E) and failed to influence the migratory capacity in transwell assays as seen in mutp53 ${ }^{\mathrm{R} 248 \mathrm{~W}-}$ containing MIA-PACA-2 cells (compare Figure 3A and 3C). However, in principle, the mutp53 ${ }^{\text {R282W}}$-pSTAT3 complex confirms a point made in our colorectal carcinoma study that the ability of mutp53 to bind pSTAT3 correlates with the degree of its stabilization (Schulz-Heddergott et al., 2018). The function which is acquired by the mutp53 ${ }^{\mathrm{R} 282 \mathrm{~W}}$-pSTAT3 complex in PA-TU-8988T remains speculative. STAT3 is not just an important factor for PDAC migration (Cardoso et al., 2012; Patel et al., 2014; Nagathihalli et al., 2016), but is also involved in many other hallmarks of cancer to promote tumor progression (Siveen et al., 2014; Zou et al., 2020).

Thus, we find that different p53 mutants have different impacts on migration- and cell growthassociated STAT3 functions. Importantly, among TP53 mutations, several other common alterations exist that drive PDAC (Bailey et al., 2016). We cannot exclude that molecular PDAC subtypes influence mutp53 GOF activities. Other mutations and alteration might also contribute to migratory differences after depletion of mutp53 variants. To address this question, an isogenic cell panel with various TP53 mutations is necessary. Since the maintenance of the TP53 copy 
number is very crucial in relation to mutp53 protein stabilization, a CRISPR/Cas9-based isogenic cell panel might be most useful.

Mechanistically, the favored gain-of-function hypothesis is that the nuclear presence of highly abundant stabilized mutp53 proteins, which have lost specific DNA binding capacity on their own, results in hijacking of (by binding to) other transcription factors and their specific cofactors, thereby building a complex network to divert and oncogenically reprogram their transcriptional activity (Muller and Vousden, 2013; 2014; Kim et al., 2015; Walerych et al., 2015; Bellazzo et al., 2018; Kim and Lozano, 2018; Mantovani et al., 2019). Regarding co-factors, it is conceivable that the mutp53 protein also adds p53-specific coactivators into this illegitimate mix, and/or that the canonical coactivator specific for the partnering transcription factor might get displaced. Thus, interplay networks of mutp53 with co-regulation of various tumor drivers is essential for GOF mediated cancer progression (Oren and Rotter, 2010; Muller and Vousden, 2013; Kim et al., 2015; Kim and Lozano, 2018; Grzes et al., 2020). This concept could explain why the mutp53 status or the status of STAT3 phosphorylation alone is not yet a determinant for migration but depends on the specific missense mutation, resulting in specific mutp53-STAT3 complexes with mutp53 variant-specific transcriptional cofactors. In line, it is shown that mutp53 ${ }^{\mathrm{R} 273 \mathrm{H}}$ and mutp53 ${ }^{\mathrm{R} 175 \mathrm{H}}$ can regulate NF-KB activity in cancer cells (Weisz et al., 2007; Cooks et al., 2013). Interestingly, NFKB and STAT3 also physically interact and coregulate transcriptional pathways in cancer (Grivennikov and Karin, 2010; Ji et al., 2019). Together with our finding that mutp53 ${ }^{\mathrm{R} 273 \mathrm{H}}$ does not significantly bind to pSTAT3 in PANC-1 cells (Figures 4B) and does not regulate their migration (Figures 3B, G), it further emphasizes the allele specificity of oncogenic mechanisms. Other studies also show context-dependent mutp53 specificities (Freed-Pastor and Prives, 2012; Kim and Lozano, 2018). One example is mutp53 ${ }^{\mathrm{R} 175 \mathrm{H}}$ which promotes aberrant self-renewal in leukemic cells through binding to $\mathrm{FOXH1}$ as critical regulator of stem cell-associated genes (Loizou et al., 2019). Furthermore, mutp53 ${ }^{\mathrm{R} 175 \mathrm{H}}$ or mutp53 ${ }^{\mathrm{R} 273 \mathrm{H} / \mathrm{C}}$ form complexes with NF-Y and p300 proteins to override cellular failsafe programs thus, permitting tumor progression (Di Agostino et al., 2006). Mutp53 promotes invasion, e.g. via constitutive activation of EGFR/integrin signaling (Muller et al., 2009) and by antagonizing TAp63 (Adorno et al., 2009).

Mutp53 stabilization occurs via binding to Hsp90 (Alexandrova et al., 2015; Mantovani et al., 2019) which offers therapeutic approaches to target stabilized GOF mutp53 protein in cancer cells via Hsp90 inhibition. Thus, treatment with the HSP90 inhibitors Ganetespib and Onalespib diminished mutp53 levels in most analyzed PDAC cells (Figure 2A). However, in BXPC-3 cells, both Hsp90 inhibitors failed to destabilize Hsp90 clients (also see functional control AKT). Why 
Klemke et al., manuscript provisionally accepted by Frontiers in Oncology (April 2021)

remains speculative but resistance mechanisms are known such as an UGT1A (UDP glucuronosyltransferase 1A) overexpression (Landmann et al., 2014). Importantly, in cells with a strong stabilization of mutp53 (MIA-PACA-2, PA-TU-8902 and PA-TU-8988T, Figure 1B), inhibition of HSP90 resulted in significant suppression of cell growth (Figure 2B). In CAPAN-1 cells with a low degree of mutp53 stabilization (Figures 1A, B), HSP90 inhibition did not substantially impact cell confluency (Figure 2B).

In sum, our preliminary in vitro results support a GOF of mutp53 ${ }^{\mathrm{R} 248 \mathrm{~W}}$ in pancreatic cancer, justifying future in vivo investigations on stabilized mutp53 as putative therapeutic target in this important tumor entity that is in dire need of new therapeutic concepts. 


\section{MATERIAL AND METHODS}

All materials used and corresponding information are provided as supplemental Table 1.

\section{Cell Culture}

Homozygous mutant human pancreatic cancer cell lines MIA-PACA-2 (mutp53 ${ }^{\text {R248W) }}$ (DZMS, RRID:CVCL_0428), PANC-1 (mutp53 ${ }^{\text {R273H }}$ ) (ATCC, RRID:CVCL_0480), BXPC-3 (mutp53 ${ }^{\text {Y220C }}$ ) (ATCC, RRID:CVCL_0186), and PA-TU-8902 (mutp53 ${ }^{\mathrm{C} 176 S}$ ) (DSMZ, RRID:CVCL_1845) were grown in DMEM (Gibco) with 10\% FBS (Merck). PA-TU-8988T (mutp53 ${ }^{\mathrm{R} 282 \mathrm{~W}}$ ) (DSMZ, RRID:CVCL_1847) were grown in DMEM medium with 5\% FBS. CAPAN-1 (mutp53 ${ }^{\text {A159V }}$ ) (ATCC, RRID:CVCL_0237) were grown in RPMI 1640 (Gibco) with 20\% FBS, and L3.6pl cells (truncating frameshift p53 mutation) (Bruns et al., 1999; Herreros-Villanueva et al., 2013) were grown in RPMI 1640 with 10\% FBS. All media were supplemented with Penicillin-Streptomycin $(10,000 \mathrm{U} / \mathrm{mL}$, Gibco) and L-Glutamine (Gibco). All cell lines were grown at $37^{\circ} \mathrm{C}$ at $5 \% \mathrm{CO}_{2}$ in a humidified atmosphere and tested for Mycoplasma contamination on a regular basis (Mycoplasma Detection Kit, Lonza). Cell line authentication certificates are provided as supplemental material.

\section{Transfection with siRNA}

Depletion of human TP53 or STAT3 mRNAs was achieved by siRNA transfection using Lipofectamine $^{\text {TM }} 3000$ (Invitrogen) or Lipofectamine ${ }^{\text {TM }} 2000$ (Invitrogen) transfection reagents. siRNA sequences are listed in supplemental Table 1. Cells were reverse transfected in 6-well plates (Sarstedt) according to manufacturer guidelines. After 24 hrs supernatant was collected and replaced by fresh culture medium. $72 \mathrm{hrs}$ post-transfection cells were harvested for analyses.

\section{Immunoblot analysis}

Cell lysates were prepared with RIPA buffer containing $20 \mathrm{mM}$ Tris-HCl pH 7.5, $10 \mathrm{mM}$ EDTA, $1 \%$ sodium deoxycholate, $150 \mathrm{mM} \mathrm{NaCl}, 1 \%$ Triton $\mathrm{X}-100,0.1 \%$ SDS, phosphatase inhibitor consisting of $2 \mathrm{mM}$ imidazol, $1 \mathrm{mM}$ sodium orthovanadate and $1 \mathrm{mM}$ sodium fluoride, and cOmplete $^{\mathrm{TM}}$ mini protease inhibitor cocktail (Roche). Samples were lysed in RIPA buffer with sonication. Protein concentrations were determined by BCA protein assay (Pierce). Equal amounts of lysates were loaded $(15-30 \mu \mathrm{g})$ and separated by SDS-polyacrylamide gel electrophoresis followed by transfer onto nitrocellulose membranes (Amersham). After blocking with 5\% milk (Roth), membranes were incubated with the following antibodies: HSC70 [B-6] (Santa Cruz), beta-Actin (Abcam), total-AKT [D9E] (Cell Signaling), p53 [DO-1] or HRP- 
conjugated p53 [DO-1] (Santa Cruz), phospho-Y705 STAT3 [EP2147Y] (Abcam), total STAT3 (Santa Cruz) or total STAT3 [79D7] (Cell Signaling), MDM2 [IF-2] (Calbiochem®/Millipore), p21 Waf1/Cip1 [12D1] (Cell Signaling). Primary antibodies were detected with HRP-conjugated secondary antibodies. Signal was developed using Clarity Max ${ }^{\mathrm{TM}}$ Western ECL Substrate (BioRad), SuperSignal ${ }^{\mathrm{TM}}$ West Femto Maximum Sensitivity Substrate (ThermoFisher Scientific) or Immobilion Western chemiluminescent HRP substrate (Millipore/Merck). For antibody details see Table 1.

\section{Co-immunoprecipitation}

For colP cells were lysed in buffer containing $50 \mathrm{mM}$ Tris- $\mathrm{HCl}, \mathrm{pH} 7.5,150 \mathrm{mM} \mathrm{NaCl}, 1 \%$ Nonidet $^{\mathrm{TM}} \mathrm{P} 40,10 \mu \mathrm{M}$ MG-132, phosphatase inhibitor consisting of $2 \mathrm{mM}$ Imidazol, $1 \mathrm{mM}$ sodium orthovanadate and $1 \mathrm{mM}$ Sodium Fluoride, and cOmplete ${ }^{\mathrm{TM}}$ mini protease inhibitor cocktail (Roche), followed by sonication. After centrifugation samples were precleared with protein $G$ Sepharose (GE Healthcare) and equal amounts of protein were immunoprecipitated using antibodies against total STAT3 (Santa Cruz), phospho-Y705 STAT3 (Abcam) or control lgG antibody (Abcam). Precipitates were analyzed by immunoblotting. For colPs, p53 was immunoblotted with an HRP-conjugated p53 antibody (Santa Cruz). 5\% of each input was used as input control and stained with beta-Actin (Abcam) as loading control. To stimulate STAT3, cells were treated with $50 \mathrm{ng} / \mathrm{mL}$ IL-6 or OSM $24 \mathrm{hrs}$ prior to performing the ColP.

\section{Cycloheximide chase}

To evaluate the stability of different mutp53 proteins in the panel of PDAC cell lines Cycloheximide $(\mathrm{CHX})$ chase experiments were performed. Cells were treated with $40 \mu \mathrm{g} / \mathrm{mL}$ Cycloheximide (Sigma-Aldrich) or ethanol vehicle control for $8 \mathrm{~h}$ and $24 \mathrm{hrs}$. Protein lysates were prepared with RIPA buffer as described in immunoblot analysis.

\section{Treatment with Hsp90 inhibitors and STAT3 inhibitor}

To investigate HSP90 chaperone dependent stabilization of different mutp53 proteins, cells were treated with Hsp90 ATPase inhibitors Ganetespib (Synta Pharmaceuticals) or Onalespib (Selleckchem). To investigate dependency on STAT3, small-molecule inhibitor Stattic (Santa Cruz) was used which prevents STAT3 phosphorylation and activation. Cells were seeded in 6well plates (Sarstedt) and treated with the inhibitors or respective DMSO control for $24 \mathrm{hrs}$ followed by harvesting protein lysates and immunoblot analysis. To determine cell confluency, 
cells were seeded in 96-wells (Corning) and treated with Onalespib or Ganetespib for 24 hrs. Confluency was determined using the Celigo Imaging Cytometer (Nexcelom, Software v5.0.0.0).

\section{Treatment with cytokines (IL-6, OSM)}

To stimulate the STAT3 pathway, cells were seeded in 6-well plates (Sarstedt) and treated with Interleukin-6 (IL-6) or Oncostatin M (OSM 209a.a.) (both from Immunotools) or solvent control for $24 \mathrm{hrs}$ and analyzed by immunoblots.

\section{Cell viability assay after Stattic treatment}

Cells were seeded in 96-well plates (Corning) and treated with increasing concentrations (0$80 \mu \mathrm{M}$ ) of Stattic or solvent control for $24 \mathrm{hrs}$. The CellTiter-Gloß Luminescent Cell Viability Assay (Promega), based on detectable ATP, was performed according to manufacturer's guidelines. Each biological replicate was measured in triplicates and viability was calculated relative to the solvent control for each cell line.

\section{Wound healing assay}

$24 \mathrm{hrs}$ after transfection with siRNAs or scrambled control, cells were incubated in serum-reduced media (1\% FBS). $48 \mathrm{hrs}$ post transfection, three scratches per well were made with a $1 \mathrm{ml}$ pipette tip or $200 \mu l$ pipette tip as dublicates. 48 hrs after scratching, at least 5 images per scratch were taken, quantified, and averaged per experiment. The degree of wound healing was determined by measuring the scratched area per image using the 'polygon selection function' of Image $J$ software. Wound healing rate was measured by averaging each scratch area after 48 hrs relative to the initial area at $0 \mathrm{hrs}$. Biological replicates are defined as independent experiments with cells at different passages and different days. For technical replicates cells from one experiment were seeded in two different wells (duplicates).

\section{Transwell migration assay}

Cells were either transfected with siRNA against TP53 mRNA, STAT3 mRNA or scrambled control, or treated with STAT3 inhibitor Stattic. $72 \mathrm{hrs}$ after siRNA transfection, cells were trypsinized and seeded into transwell inserts (Corning) in serum-reduced media (1\% FBS for MIAPACA-2, PANC-1, BXPC-3 and PA-TU-8902; 0.5\% FBS for PA-TU-8988T). Wells (Corning) were filled with the respective complete medium of each cell line. 
To investigate migration potential upon Stattic treatment, cells were seeded in transwell inserts as described above. Different concentrations of Stattic or respective control were added to the cells 1-2 hrs after seeding, allowing cells to settle before treatment.

Final $24 \mathrm{hrs}$ after seeding, cells that had migrated to the underside of the membrane were carefully washed with PBS, fixed in ice cold methanol for 10 min and stained with crystal violet $(0.1 \%$ in $20 \% \mathrm{EtOH})$ for $20 \mathrm{~min}$. After washing, remaining cells inside the insert were removed using a pre-wet Q-tip. Migrated cells were visualized by light microscopy and analyzed using Image J. Migration rate was calculated relative to scrambled siRNA or solvent control, respectively. Biological replicates are defined as independent experiments with cells at different passages and different days. For technical replicates cells from one experiment were seeded in two different transwell inserts (dublicates).

Attempting to induce migration of PA-TU-8902 cells, cells were seeded in 6-well plates (Corning) and treated with $50 \mathrm{ng} / \mathrm{mL}$ IL-6 or OSM (Immunotools). After $24 \mathrm{hrs}$ pre-treatment, cells were transferred to transwell inserts, cytokines added again. And followed as described above.

\section{Analysis of human patient TCGA data}

Human genomic data including TP53 gene mutation and clinical information was downloaded from cBioPortal (www.cbioportal.org). We used cBioportal Pancreatic ductal adenocarcinoma database in this analysis (Cerami et al., 2012; Gao et al., 2013). Two datasets were used to detect mutated samples and the clinical data, QCMG, Nature 2016, and TCGA, PanCancer Atlas (Bailey et al., 2016; Cancer Genome Atlas Research Network. Electronic address and Cancer Genome Atlas Research, 2017). TP53 R248Q/W missense mutant group was sampled with TP53 missense mutations (MS) with amino acid change R248, and TP53 LOF group was sampled with frameshift (FS) and nonsense (NS) TP53 mutations. R language (The R Project for Statistical Computing, https://www.r-project.org, version 4.0.2) and the package "survival" were used in the analysis, including calculating log-rank p-value and Kaplan-Meier curves.

\section{Statistical analysis}

The number of biological and technical replicates (mean \pm SEM) is provided in the figure legends. For all experiments, an unpaired Student's t test was used to calculate p-values. 
Klemke et al., manuscript provisionally accepted by Frontiers in Oncology (April 2021)

\section{Acknowledgement}

We thank Jinyu Li (Stony Brook University Cancer Center) for statistical analysis of TCGA patient data. We thank Silke Kaulfuß (Institute of Human Genetics, University Medical Center Göttingen, Germany) for sequencing of the human cell line L3.6pl. 


\section{REFERENCES}

Adorno, M., Cordenonsi, M., Montagner, M., Dupont, S., Wong, C., Hann, B., et al. (2009). A Mutant-p53/Smad complex opposes p63 to empower TGFbeta-induced metastasis. Cell 137(1), 87-98. doi: 10.1016/j.cell.2009.01.039.

Alexandrova, E.M., Mirza, S.A., Xu, S., Schulz-Heddergott, R., Marchenko, N.D., and Moll, U.M. (2017). p53 loss-of-heterozygosity is a necessary prerequisite for mutant p53 stabilization and gain-of-function in vivo. Cell Death Dis 8(3), e2661. doi: 10.1038/cddis.2017.80.

Alexandrova, E.M., Yallowitz, A.R., Li, D., Xu, S., Schulz, R., Proia, D.A., et al. (2015). Improving survival by exploiting tumour dependence on stabilized mutant p53 for treatment. Nature 523(7560), 352-356. doi: 10.1038/nature14430.

American Cancer Society (2020). Survival Rates for Pancreatic Cancer [Online]. Available: https://www.cancer.org/cancer/pancreatic-cancer/detection-diagnosis-staging/survivalrates.html\# [Accessed 10.12.2020].

Bailey, P., Chang, D.K., Nones, K., Johns, A.L., Patch, A.M., Gingras, M.C., et al. (2016). Genomic analyses identify molecular subtypes of pancreatic cancer. Nature 531(7592), 47-52. doi: 10.1038 /nature16965.

Bellazzo, A., Sicari, D., Valentino, E., Del Sal, G., and Collavin, L. (2018). Complexes formed by mutant $\mathrm{p} 53$ and their roles in breast cancer. Breast Cancer (Dove Med Press) 10, 101-112. doi: $10.2147 /$ BCTT.S145826.

Brosh, R., and Rotter, V. (2009). When mutants gain new powers: news from the mutant p53 field. Nat Rev Cancer 9(10), 701-713. doi: 10.1038/nrc2693.

Bruns, C.J., Harbison, M.T., Kuniyasu, H., Eue, I., and Fidler, I.J. (1999). In vivo selection and characterization of metastatic variants from human pancreatic adenocarcinoma by using orthotopic implantation in nude mice. Neoplasia 1(1), 50-62. doi: 10.1038/sj.neo.7900005.

Bullock, A.N., Henckel, J., and Fersht, A.R. (2000). Quantitative analysis of residual folding and DNA binding in mutant p53 core domain: definition of mutant states for rescue in cancer therapy. Oncogene 19(10), 1245-1256. doi: 10.1038/sj.onc.1203434.

Cancer Genome Atlas Research Network. Electronic address, a.a.d.h.e., and Cancer Genome Atlas Research, N. (2017). Integrated Genomic Characterization of Pancreatic Ductal Adenocarcinoma. Cancer Cell 32(2), 185-203 e113. doi: 10.1016/j.ccell.2017.07.007.

Cardoso, A.A., Jiang, Y., Luo, M., Reed, A.M., Shahda, S., He, Y., et al. (2012). APE1/Ref-1 regulates STAT3 transcriptional activity and APE1/Ref-1-STAT3 dual-targeting effectively inhibits pancreatic cancer cell survival. PLoS One 7(10), e47462. doi: 10.1371/journal.pone.0047462.

Cerami, E., Gao, J., Dogrusoz, U., Gross, B.E., Sumer, S.O., Aksoy, B.A., et al. (2012). The cBio cancer genomics portal: an open platform for exploring multidimensional cancer genomics data. Cancer Discov 2(5), 401-404. doi: 10.1158/2159-8290.CD-12-0095.

Che, Q., Xiao, X., Liu, M., Lu, Y., Dong, X., and Liu, S. (2019). IL-6 promotes endometrial cancer cells invasion and migration through signal transducers and activators of transcription 3 signaling pathway. Pathol Res Pract 215(6), 152392. doi: 10.1016/j.prp.2019.03.020. 
Cicenas, J., Kvederaviciute, K., Meskinyte, I., Meskinyte-Kausiliene, E., Skeberdyte, A., and Cicenas, J. (2017). KRAS, TP53, CDKN2A, SMAD4, BRCA1, and BRCA2 Mutations in Pancreatic Cancer. Cancers (Basel) 9(5). doi: 10.3390/cancers9050042.

Cooks, T., Pateras, I.S., Tarcic, O., Solomon, H., Schetter, A.J., Wilder, S., et al. (2013). Mutant p53 prolongs NF-kappaB activation and promotes chronic inflammation and inflammationassociated colorectal cancer. Cancer Cell 23(5), 634-646. doi: 10.1016/j.ccr.2013.03.022.

Denley, S.M., Jamieson, N.B., McCall, P., Oien, K.A., Morton, J.P., Carter, C.R., et al. (2013). Activation of the IL-6R/Jak/stat pathway is associated with a poor outcome in resected pancreatic ductal adenocarcinoma. J Gastrointest Surg 17(5), 887-898. doi: 10.1007/s11605-013-2168-7.

Di Agostino, S., Strano, S., Emiliozzi, V., Zerbini, V., Mottolese, M., Sacchi, A., et al. (2006). Gain of function of mutant $\mathrm{p53}$ : the mutant $\mathrm{p} 53 / \mathrm{NF}-\mathrm{Y}$ protein complex reveals an aberrant transcriptional mechanism of cell cycle regulation. Cancer Cell 10(3), 191-202. doi: 10.1016/j.ccr.2006.08.013.

Freed-Pastor, W.A., and Prives, C. (2012). Mutant p53: one name, many proteins. Genes Dev 26(12), 1268-1286. doi: 10.1101/gad.190678.112.

Gao, J., Aksoy, B.A., Dogrusoz, U., Dresdner, G., Gross, B., Sumer, S.O., et al. (2013). Integrative analysis of complex cancer genomics and clinical profiles using the cBioPortal. Sci Signal 6(269), pl1. doi: 10.1126/scisignal.2004088.

Gencel-Augusto, J., and Lozano, G. (2020). p53 tetramerization: at the center of the dominantnegative effect of mutant p53. Genes Dev 34(17-18), 1128-1146. doi: 10.1101/gad.340976.120.

Goh, A.M., Coffill, C.R., and Lane, D.P. (2011). The role of mutant p53 in human cancer. J Pathol 223(2), 116-126. doi: 10.1002/path.2784.

Grasso, C., Jansen, G., and Giovannetti, E. (2017). Drug resistance in pancreatic cancer: Impact of altered energy metabolism. Crit Rev Oncol Hematol 114, 139-152. doi: 10.1016/j.critrevonc.2017.03.026.

Grivennikov, S.I., and Karin, M. (2010). Dangerous liaisons: STAT3 and NF-kappaB collaboration and crosstalk in cancer. Cytokine Growth Factor Rev 21(1), 11-19. doi: 10.1016/j.cytogfr.2009.11.005.

Grzes, M., Oron, M., Staszczak, Z., Jaiswar, A., Nowak-Niezgoda, M., and Walerych, D. (2020). A Driver Never Works Alone-Interplay Networks of Mutant p53, MYC, RAS, and Other Universal Oncogenic Drivers in Human Cancer. Cancers (Basel) 12(6). doi: 10.3390/cancers12061532.

Guo, J., Xie, K., and Zheng, S. (2016). Molecular Biomarkers of Pancreatic Intraepithelial Neoplasia and Their Implications in Early Diagnosis and Therapeutic Intervention of Pancreatic Cancer. Int J Biol Sci 12(3), 292-301. doi: 10.7150/ijbs.14995.

Hanel, W., Marchenko, N., Xu, S., Yu, S.X., Weng, W., and Moll, U. (2013). Two hot spot mutant p53 mouse models display differential gain of function in tumorigenesis. Cell Death Differ 20(7), 898-909. doi: 10.1038/cdd.2013.17.

Herreros-Villanueva, M., Zhang, J.S., Koenig, A., Abel, E.V., Smyrk, T.C., Bamlet, W.R., et al. (2013). SOX2 promotes dedifferentiation and imparts stem cell-like features to pancreatic cancer cells. Oncogenesis 2, e61. doi: 10.1038/oncsis.2013.23. 
Hollstein, M., Sidransky, D., Vogelstein, B., and Harris, C.C. (1991). p53 mutations in human cancers. Science 253(5015), 49-53. doi: 10.1126/science.1905840.

Ji, Z., He, L., Regev, A., and Struhl, K. (2019). Inflammatory regulatory network mediated by the joint action of NF-kB, STAT3, and AP-1 factors is involved in many human cancers. Proc Natl Acad Sci U S A 116(19), 9453-9462. doi: 10.1073/pnas.1821068116.

Kadosh, E., Snir-Alkalay, I., Venkatachalam, A., May, S., Lasry, A., Elyada, E., et al. (2020). The gut microbiome switches mutant p53 from tumour-suppressive to oncogenic. Nature 586(7827), 133-138. doi: 10.1038/s41586-020-2541-0.

Kim, M.P., and Lozano, G. (2018). Mutant p53 partners in crime. Cell Death Differ 25(1), 161-168. doi: 10.1038/cdd.2017.185.

Kim, M.P., Zhang, Y., and Lozano, G. (2015). Mutant p53: Multiple Mechanisms Define Biologic Activity in Cancer. Front Oncol 5, 249. doi: 10.3389/fonc.2015.00249.

Klemke, L., De Oliveira, T., Witt, D., Winkler, N., Bohnenberger, H., Bucala, R., et al. (2021). Hsp90stabilized MIF supports tumor progression via macrophage recruitment and angiogenesis in colorectal cancer. Cell Death Dis 12(2), 155. doi: 10.1038/s41419-021-03426-z.

Landmann, H., Proia, D.A., He, S., Ogawa, L.S., Kramer, F., Beissbarth, T., et al. (2014). UDP glucuronosyltransferase $1 \mathrm{~A}$ expression levels determine the response of colorectal cancer cells to the heat shock protein 90 inhibitor ganetespib. Cell Death Dis 5, e1411. doi: 10.1038/cddis.2014.378.

Lane, D.P. (1992). Cancer. p53, guardian of the genome. Nature 358(6381), 15-16. doi: 10.1038/358015a0.

Lee, M.K., Teoh, W.W., Phang, B.H., Tong, W.M., Wang, Z.Q., and Sabapathy, K. (2012). Cell-type, dose, and mutation-type specificity dictate mutant p53 functions in vivo. Cancer Cell 22(6), 751-764. doi: 10.1016/j.ccr.2012.10.022.

Lin, L., Jou, D., Wang, Y., Ma, H., Liu, T., Fuchs, J., et al. (2016). STAT3 as a potential therapeutic target in ALDH+ and CD44+/CD24+ stem cell-like pancreatic cancer cells. Int J Oncol 49(6), 2265-2274. doi: 10.3892/ijo.2016.3728.

Lin, L., Liu, A., Peng, Z., Lin, H.J., Li, P.K., Li, C., et al. (2011). STAT3 is necessary for proliferation and survival in colon cancer-initiating cells. Cancer Res 71(23), 7226-7237. doi: 10.1158/0008-5472.CAN-10-4660.

Loizou, E., Banito, A., Livshits, G., Ho, Y.J., Koche, R.P., Sanchez-Rivera, F.J., et al. (2019). A Gainof-Function p53-Mutant Oncogene Promotes Cell Fate Plasticity and Myeloid Leukemia through the Pluripotency Factor FOXH1. Cancer Discov 9(7), 962-979. doi: 10.1158/21598290.CD-18-1391.

Mantovani, F., Collavin, L., and Del Sal, G. (2019). Mutant p53 as a guardian of the cancer cell. Cell Death Differ 26(2), 199-212. doi: 10.1038/s41418-018-0246-9.

Muller, P.A., Caswell, P.T., Doyle, B., Iwanicki, M.P., Tan, E.H., Karim, S., et al. (2009). Mutant p53 drives invasion by promoting integrin recycling. Cell 139(7), 1327-1341. doi: 10.1016/j.cell.2009.11.026.

Muller, P.A., and Vousden, K.H. (2013). p53 mutations in cancer. Nat Cell Biol 15(1), 2-8. doi: $10.1038 / \mathrm{ncb} 2641$.

Muller, P.A., and Vousden, K.H. (2014). Mutant p53 in cancer: new functions and therapeutic opportunities. Cancer Cell 25(3), 304-317. doi: 10.1016/j.ccr.2014.01.021. 
Nagathihalli, N.S., Castellanos, J.A., Lamichhane, P., Messaggio, F., Shi, C., Dai, X., et al. (2018). Inverse Correlation of STAT3 and MEK Signaling Mediates Resistance to RAS Pathway Inhibition in Pancreatic Cancer. Cancer Res 78(21), 6235-6246. doi: 10.1158/00085472.CAN-18-0634.

Nagathihalli, N.S., Castellanos, J.A., Shi, C., Beesetty, Y., Reyzer, M.L., Caprioli, R., et al. (2015). Signal Transducer and Activator of Transcription 3, Mediated Remodeling of the Tumor Microenvironment Results in Enhanced Tumor Drug Delivery in a Mouse Model of Pancreatic Cancer. Gastroenterology 149(7), 1932-1943 e1939. doi: 10.1053/j.gastro.2015.07.058.

Nagathihalli, N.S., Castellanos, J.A., VanSaun, M.N., Dai, X., Ambrose, M., Guo, Q., et al. (2016). Pancreatic stellate cell secreted IL-6 stimulates STAT3 dependent invasiveness of pancreatic intraepithelial neoplasia and cancer cells. Oncotarget 7(40), 65982-65992. doi: 10.18632/oncotarget.11786.

Natesh, K., Bhosale, D., Desai, A., Chandrika, G., Pujari, R., Jagtap, J., et al. (2015). Oncostatin-M differentially regulates mesenchymal and proneural signature genes in gliomas via STAT3 signaling. Neoplasia 17(2), 225-237. doi: 10.1016/j.neo.2015.01.001.

Oren, M., and Rotter, V. (2010). Mutant p53 gain-of-function in cancer. Cold Spring Harb Perspect Biol 2(2), a001107. doi: 10.1101/cshperspect.a001107.

Orth, M., Metzger, P., Gerum, S., Mayerle, J., Schneider, G., Belka, C., et al. (2019). Pancreatic ductal adenocarcinoma: biological hallmarks, current status, and future perspectives of combined modality treatment approaches. Radiat Oncol 14(1), 141. doi: 10.1186/s13014019-1345-6.

Parrales, A., Ranjan, A., Iyer, S.V., Padhye, S., Weir, S.J., Roy, A., et al. (2016). DNAJA1 controls the fate of misfolded mutant p53 through the mevalonate pathway. Nat Cell Biol 18(11), 1233-1243. doi: 10.1038/ncb3427.

Patel, K., Kollory, A., Takashima, A., Sarkar, S., Faller, D.V., and Ghosh, S.K. (2014). MicroRNA let7 downregulates STAT3 phosphorylation in pancreatic cancer cells by increasing SOCS3 expression. Cancer Lett 347(1), 54-64. doi: 10.1016/j.canlet.2014.01.020.

Razidlo, G.L., Burton, K.M., and McNiven, M.A. (2018). Interleukin-6 promotes pancreatic cancer cell migration by rapidly activating the small GTPase CDC42. J Biol Chem 293(28), 1114311153. doi: 10.1074/jbc.RA118.003276.

Sabapathy, K., and Lane, D.P. (2018). Therapeutic targeting of p53: all mutants are equal, but some mutants are more equal than others. Nat Rev Clin Oncol 15(1), 13-30. doi: 10.1038/nrclinonc.2017.151.

Said, R., Hong, D.S., Warneke, C.L., Lee, J.J., Wheler, J.J., Janku, F., et al. (2013). P53 mutations in advanced cancers: clinical characteristics, outcomes, and correlation between progression-free survival and bevacizumab-containing therapy. Oncotarget 4(5), 705-714. doi: 10.18632/oncotarget.974.

Schulz-Heddergott, R., and Moll, U.M. (2018). Gain-of-Function (GOF) Mutant p53 as Actionable Therapeutic Target. Cancers (Basel) 10(6). doi: 10.3390/cancers10060188.

Schulz-Heddergott, R., Stark, N., Edmunds, S.J., Li, J., Conradi, L.C., Bohnenberger, H., et al. (2018). Therapeutic Ablation of Gain-of-Function Mutant p53 in Colorectal Cancer Inhibits Stat3Mediated Tumor Growth and Invasion. Cancer Cell 34(2), 298-314 e297. doi: 10.1016/j.ccell.2018.07.004. 
Schust, J., Sperl, B., Hollis, A., Mayer, T.U., and Berg, T. (2006). Stattic: a small-molecule inhibitor of STAT3 activation and dimerization. Chem Biol 13(11), 1235-1242. doi: 10.1016/j.chembiol.2006.09.018.

Siegel, R.L., Miller, K.D., and Jemal, A. (2020). Cancer statistics, 2020. CA Cancer J Clin 70(1), 7-30. doi: 10.3322/caac. 21590.

Siveen, K.S., Sikka, S., Surana, R., Dai, X., Zhang, J., Kumar, A.P., et al. (2014). Targeting the STAT3 signaling pathway in cancer: role of synthetic and natural inhibitors. Biochim Biophys Acta 1845(2), 136-154. doi: 10.1016/j.bbcan.2013.12.005.

Smigiel, J.M., Parameswaran, N., and Jackson, M.W. (2018). Targeting Pancreatic Cancer Cell Plasticity: The Latest in Therapeutics. Cancers (Basel) 10(1). doi: 10.3390/cancers10010014.

Spitzner, M., Roesler, B., Bielfeld, C., Emons, G., Gaedcke, J., Wolff, H.A., et al. (2014). STAT3 inhibition sensitizes colorectal cancer to chemoradiotherapy in vitro and in vivo. Int J Cancer 134(4), 997-1007. doi: 10.1002/ijc.28429.

Ubby, I., Krueger, C., Rosato, R., Qian, W., Chang, J., and Sabapathy, K. (2019). Cancer therapeutic targeting using mutant-p53-specific siRNAs. Oncogene 38(18), 3415-3427. doi: 10.1038/s41388-018-0652-y.

Walerych, D., Kudla, G., Gutkowska, M., Wawrzynow, B., Muller, L., King, F.W., et al. (2004). Hsp90 chaperones wild-type p53 tumor suppressor protein. J Biol Chem 279(47), 4883648845. doi: 10.1074/jbc.M407601200.

Walerych, D., Lisek, K., and Del Sal, G. (2015). Mutant p53: One, No One, and One Hundred Thousand. Front Oncol 5, 289. doi: 10.3389/fonc.2015.00289.

Walerych, D., Olszewski, M.B., Gutkowska, M., Helwak, A., Zylicz, M., and Zylicz, A. (2009). Hsp70 molecular chaperones are required to support p53 tumor suppressor activity under stress conditions. Oncogene 28(48), 4284-4294. doi: 10.1038/onc.2009.281.

Wawrzynow, B., Zylicz, A., and Zylicz, M. (2018). Chaperoning the guardian of the genome. The two-faced role of molecular chaperones in p53 tumor suppressor action. Biochim Biophys Acta Rev Cancer 1869(2), 161-174. doi: 10.1016/j.bbcan.2017.12.004.

Weisz, L., Damalas, A., Liontos, M., Karakaidos, P., Fontemaggi, G., Maor-Aloni, R., et al. (2007). Mutant p53 enhances nuclear factor kappaB activation by tumor necrosis factor alpha in cancer cells. Cancer Res 67(6), 2396-2401. doi: 10.1158/0008-5472.CAN-06-2425.

Wiech, M., Olszewski, M.B., Tracz-Gaszewska, Z., Wawrzynow, B., Zylicz, M., and Zylicz, A. (2012). Molecular mechanism of mutant p53 stabilization: the role of HSP70 and MDM2. PLoS One 7(12), e51426. doi: 10.1371/journal.pone.0051426.

Xu, J., Wang, J., Hu, Y., Qian, J., Xu, B., Chen, H., et al. (2014). Unequal prognostic potentials of p53 gain-of-function mutations in human cancers associate with drug-metabolizing activity. Cell Death Dis 5, e1108. doi: 10.1038/cddis.2014.75.

Zhang, Y., Xiong, S., Liu, B., Pant, V., Celii, F., Chau, G., et al. (2018). Somatic Trp53 mutations differentially drive breast cancer and evolution of metastases. Nat Commun 9(1), 3953. doi: 10.1038/s41467-018-06146-9.

Zilfou, J.T., and Lowe, S.W. (2009). Tumor suppressive functions of p53. Cold Spring Harb Perspect Biol 1(5), a001883. doi: 10.1101/cshperspect.a001883.

Zou, S., Tong, Q., Liu, B., Huang, W., Tian, Y., and Fu, X. (2020). Targeting STAT3 in Cancer Immunotherapy. Mol Cancer 19(1), 145. doi: 10.1186/s12943-020-01258-7. 


\section{Figures/Figure Legends}

A
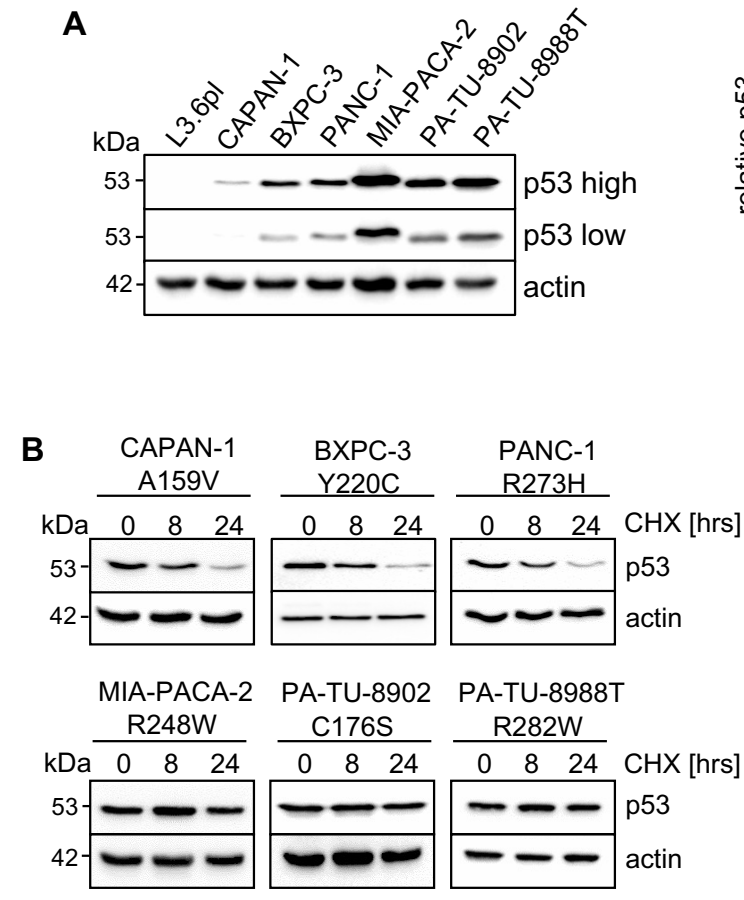
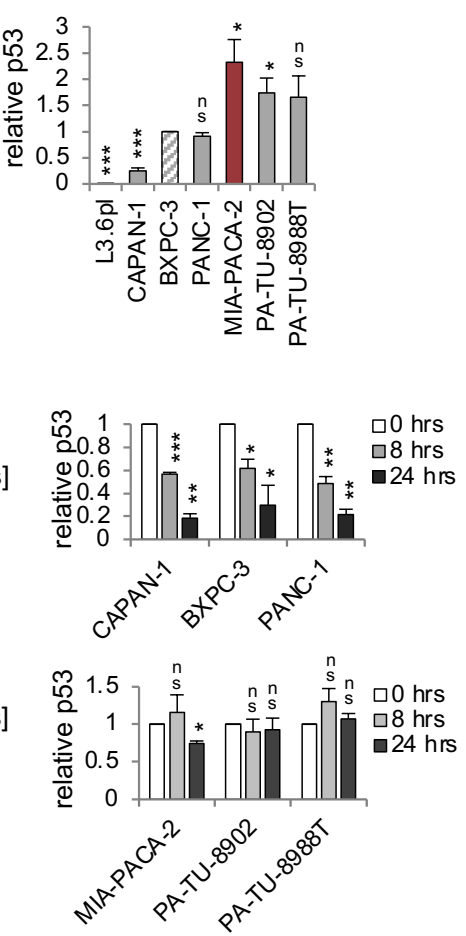

Figure 1

Figure 1: Stabilization of various missense p53 mutants in human PDAC cell lines. (A) Six PDAC cell lines harbouring various missense mutant p53 variants exhibit differential steady state protein levels. One representative immunoblot analysis out of 4 is shown. Actin as loading control. 'p53 high' and 'p53 low' mean exposure time. CAPAN-1 (mutp53 ${ }^{\mathrm{A} 159 \mathrm{~V}}$ ), BXPC-3 (mutp53 ${ }^{\mathrm{Y} 220 \mathrm{C}}$ ), PANC-1 (mutp53 ${ }^{\mathrm{R} 273 \mathrm{H}}$ ), MIA-PACA-2 (mutp53 ${ }^{\mathrm{R} 248 \mathrm{~W}}$ ), PA-TU-8902 (mutp53 ${ }^{\mathrm{C} 176 \mathrm{~S}}$ ) and PA-TU8988T (mutp53 ${ }^{\mathrm{R} 282 \mathrm{~W}}$ ). L3.6pl cells harboring a truncating LOF mutation served as p53 null control. (right) Diagrams represent the means \pm SEM of densitometric quantifications of two independent experiments with two technical replicates each (total $n=4$ immunoblots), normalized to actin or HSC70 and calculated relative to mutp53 level in BXPC-3 cells (patterned bar). (B) Differential half-lives of mutp53 proteins. Cycloheximide $(\mathrm{CHX})$ chase experiment. Cells were treated with $\mathrm{CHX}$ for 8 and $24 \mathrm{hrs}$ or vehicle control $(0 \mathrm{hrs})$. One representative immunoblot. Actin, loading control. (right) Diagrams represent mutp53 protein levels as means \pm SEM of densitometric quantifications of two independent experiments $(n=2)$, normalized to actin or HSC70. Calculated relative to control treatment $(0 \mathrm{hrs})$. (A, B) Student's t test. ${ }^{*} p \leq 0.05 ;{ }^{* *} p \leq 0.01$; ${ }^{* * *} p \leq 0.001$; ns, not significant. 

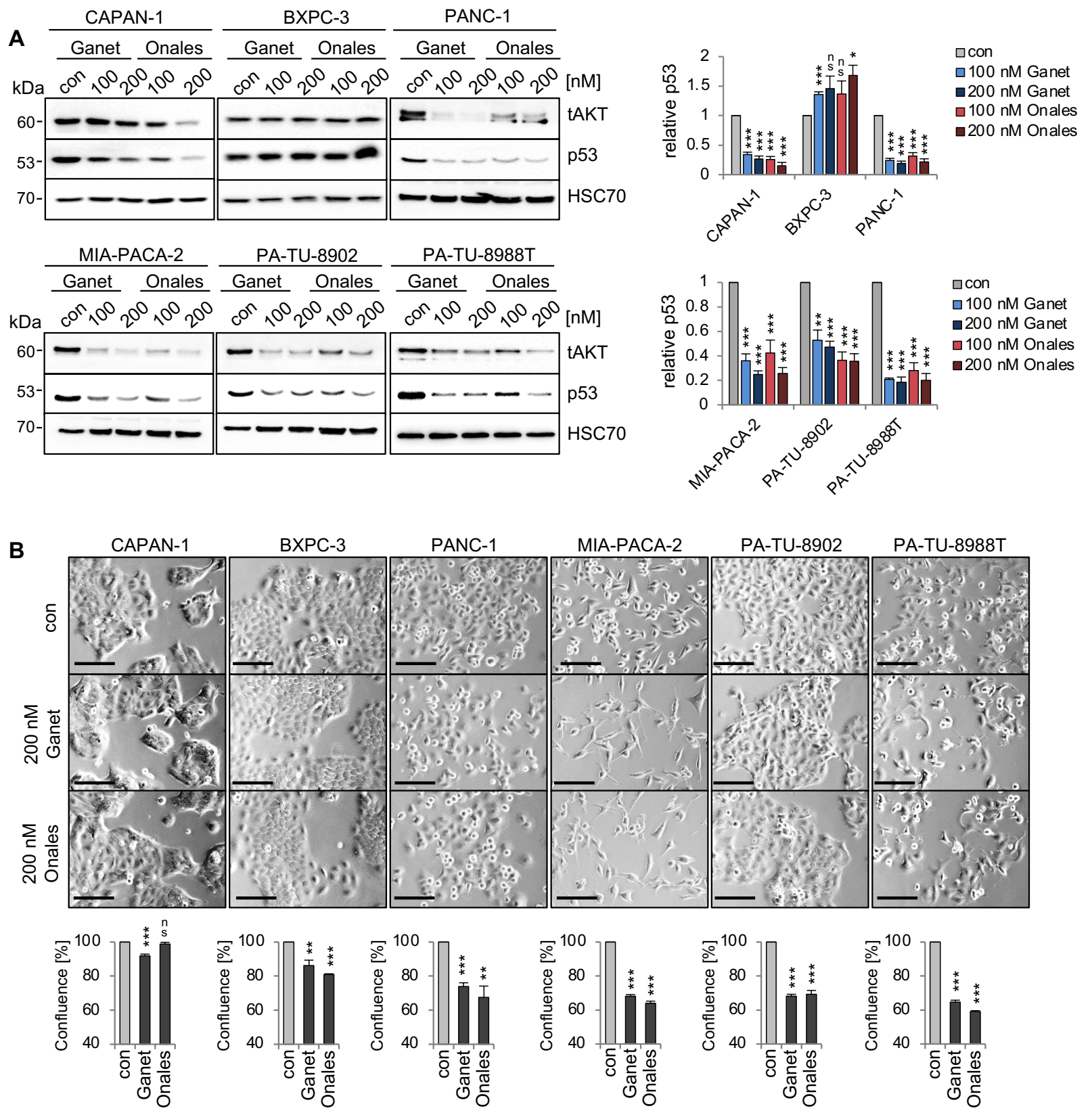

Figure 2 
Klemke et al., manuscript provisionally accepted by Frontiers in Oncology (April 2021)

Figure 2: Missense p53 mutants in PDAC cells are stabilized by HSP90. (A) Hsp90 dependent aberrant stabilization of mutp53 proteins in PDAC cell lines. Cells were treated for $24 \mathrm{hrs}$ with the indicated concentrations of Ganetespib, Onalespib or DMSO. One representative immunoblot out of 3 each is presented. HSC70, loading control. Total AKT ('tAKT', AKT serine/threonine kinase 1) as well-known Hsp90 client serves as functional control for an Hsp90 inhibition. (right) Diagrams represent the means \pm SEM of densitometric quantifications of at least two independent experiments with technical replicates (total $n \geq 3$ immunoblots), normalized to HSC70. Calculated relative to control DMSO treatments (con). (B) Cell confluence determination. Representative images of cells after treatment with $200 \mathrm{nM}$ Ganetespib, Onalespib or solvent control for $24 \mathrm{hrs}$. Cell confluency was analysed using a Celigo imaging cytometer. Scale bars, $100 \mu \mathrm{m}$. Confluence was calculated relative to their respective DMSO control from $n=3$ biological replicates. Student's t test. ${ }^{*} p \leq 0.05 ;{ }^{* *} p \leq 0.01 ;{ }^{* * *} p \leq 0.001 ;$ ns, not significant. 
A MIA-PACA-2 - mutp53R248W
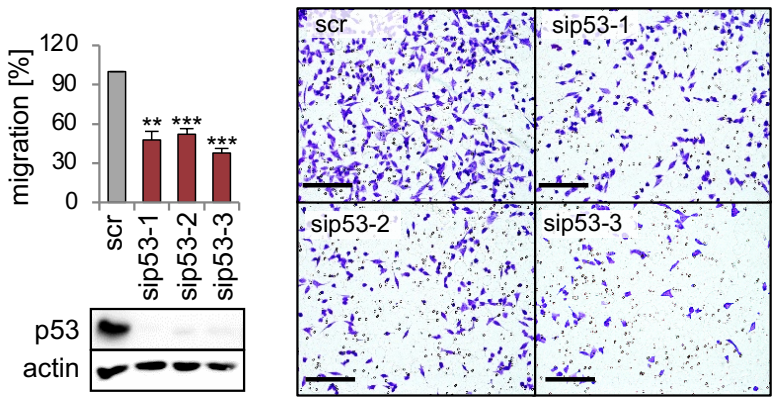

C PA-TU-8988T-mutp53R282W
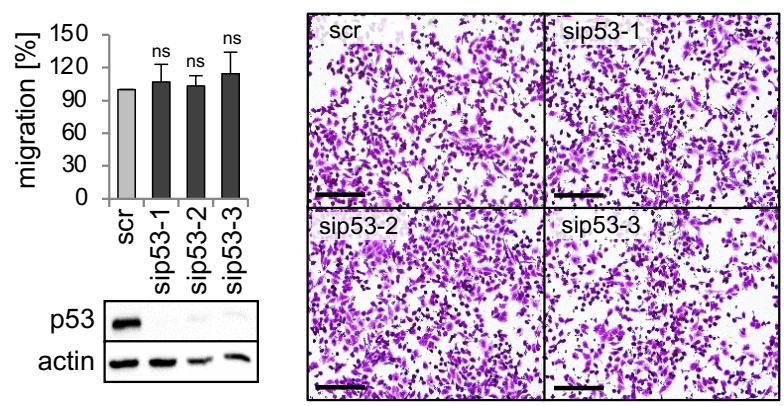

E PA-TU-8902 - mutp53C176S

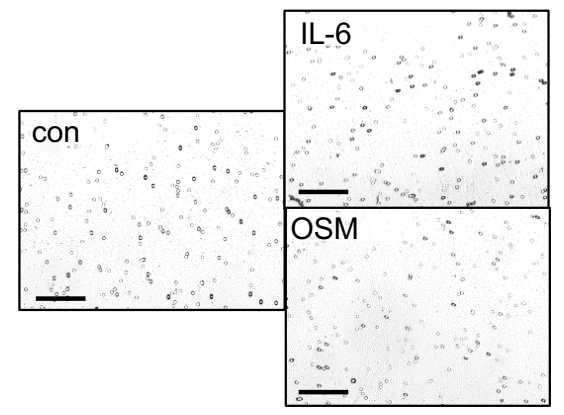

G PANC-1 - mutp53R273H
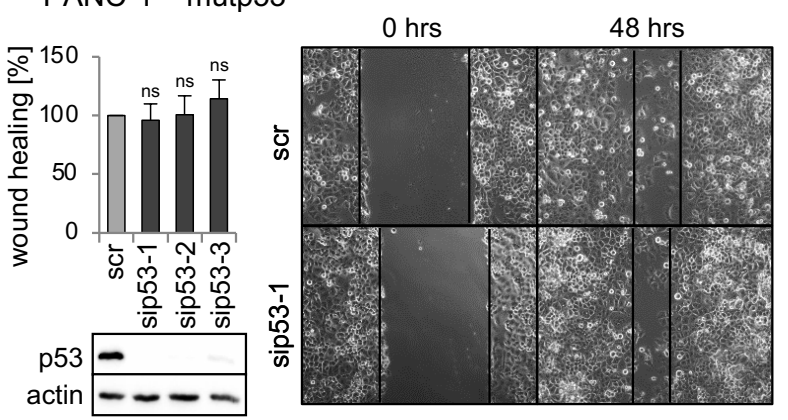

B PANC-1 - mutp53R273H
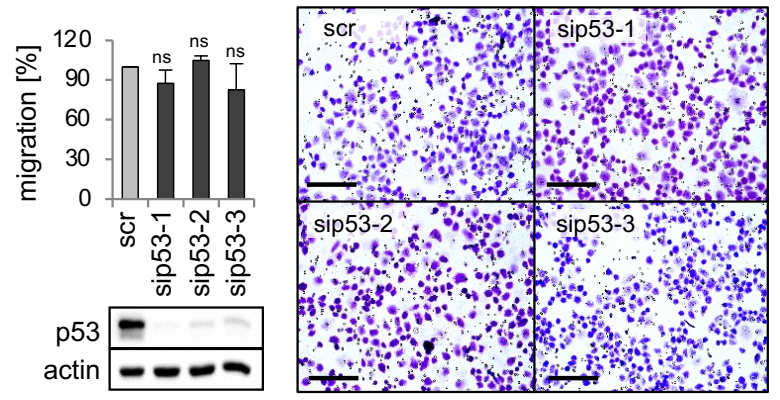

D BXPC-3 - mutp53 $3^{\mathrm{Y} 220 \mathrm{C}}$
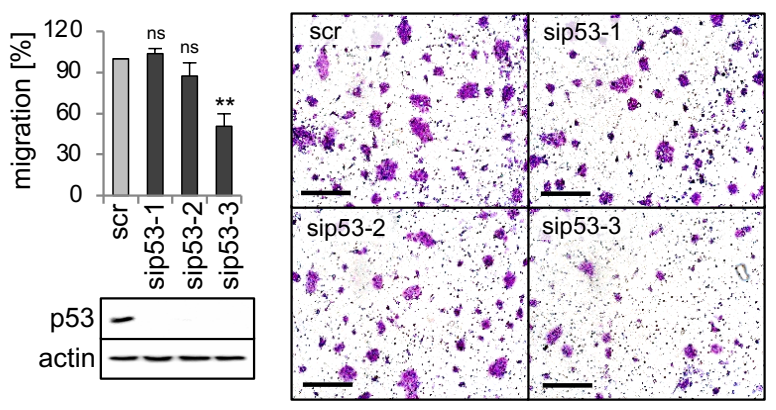

F MIA-PACA-2 - mutp53 ${ }^{\text {R248W }} 0$ hrs

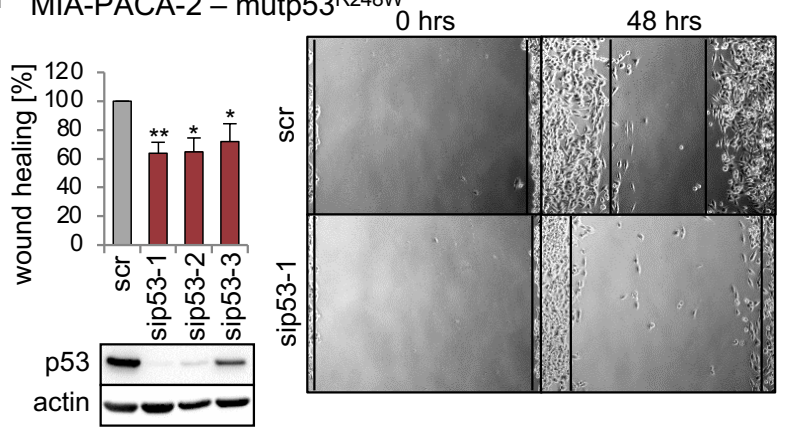

H PA-TU-8988T- mutp53 ${ }^{\text {R282W }}$
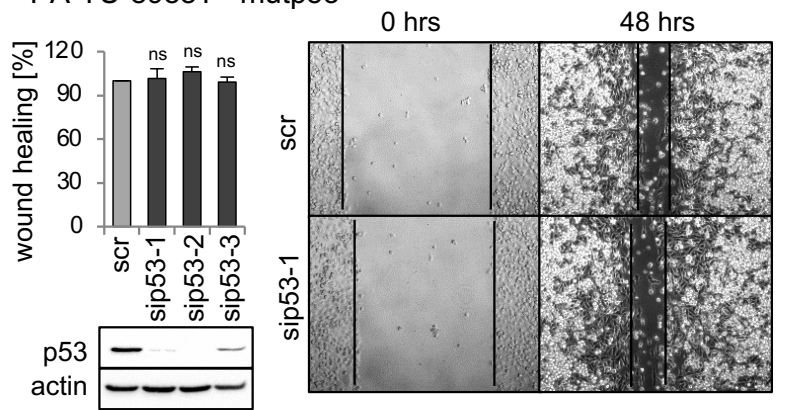

Figure 3 
Figure 3: Mutp53 ${ }^{\mathrm{R} 248 \mathrm{~W}}$ selectively promotes migration in PDAC cells. (A-D) Transwell migration assays of MIA-PACA-2, PANC-1, PA-TU-8988Tand BXPC-3 cells to evaluate mutp53dependent migration activity. mutp53 was depleted with three different siRNAs against TP53 mRNA (sip53 1-3). 72 hrs post-transfection with siRNAs, cells were seeded into transwell inserts and migration to the membrane underside was determined after 24 hrs. MIA-PACA-2 cells: 3 biological replicates $(n=3)$, PANC-1 cells: 2 biological replicates $(n=2)$, PA-TU-8988T cells: 3 biological replicates $(n=3), B X P C-3$ cells: 3 biological replicates, one with a technical replicate $(n=4)$. Note, siRNA 'sip53-3' reduced migration in BXPC-3 cells might be a consequence of siRNA off-target effects. Migration was calculated relative to scrambled control (scr, set as 100\%). Representative images of membrane undersides are shown. Scale bars, $200 \mu \mathrm{m}$. Immunoblot analysis verifies knockdown of mutp53. Actin, loading control. (E) Transwell migration assay of PA-TU-8902. Representative images of stained transwells after 24 hrs of migration are shown. To induce migration cells had been stimulated for $24 \mathrm{hrs}$ with $50 \mathrm{ng} / \mathrm{mL}$ Interleukin-6 (IL-6), Oncostatin M (OSM) or solvent control (con) prior to seeding into inserts, followed by additional cytokine treatment for another $24 \mathrm{hrs}$. Gray dots are pores of the membrane. Scale bars, $200 \mu \mathrm{m}$. (F-H) mutp53-dependent wound healing of MIA-PACA-2, PANC-1 and PA-TU-8988T cells. mutp53 knockdown for 48 hrs using three different siRNAs (sip53 1-3). 48 hrs post-transfection, scratch assays were performed for another $48 \mathrm{hrs}$. A minimum of 5 images were taken and quantified. MIA-PACA-2 cells: 3 biological replicates, 1 out of 3 with a technical replicate $(n=4)$, PANC-1 cells: 2 biological replicates, 1 out of 2 with a technical replicate $(n=3)$, PA-TU-8988T cells: 2 technical replicates $(n=2)$. Wound healing capacity was calculated relative to scrambled control (scr). Representative images after 0 hrs and 48 hrs are shown. Solid lines represent edges of the scratch. Immunoblots verify knockdown of mutp53. Actin, loading control. (A-D, F-H) Diagrams represent the means \pm SEM. Student's t test. ${ }^{*} p \leq 0.05$; ${ }^{* *} p \leq 0.01$; ${ }^{* * *} p \leq 0.001$; ns, not significant. 
A

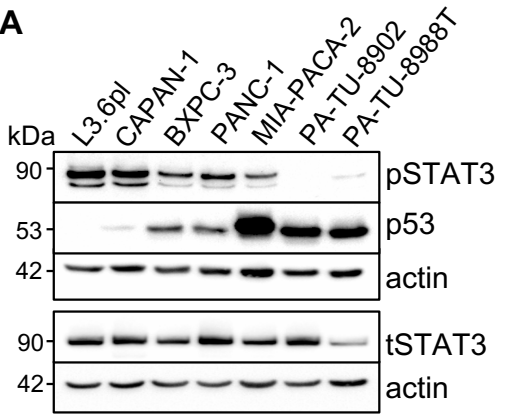

D input $\underline{\lg G}$ PSTAT3

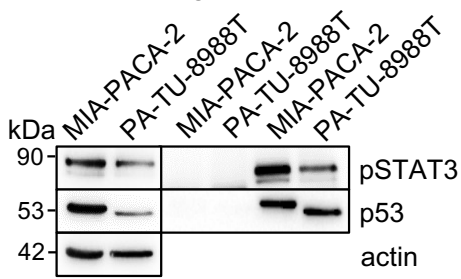

B

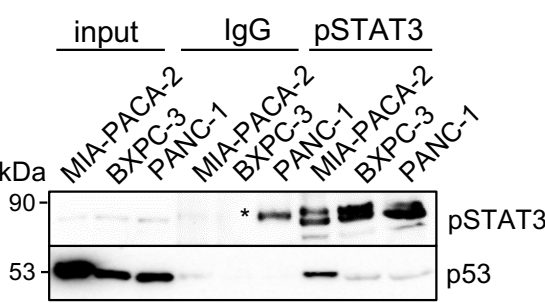

E

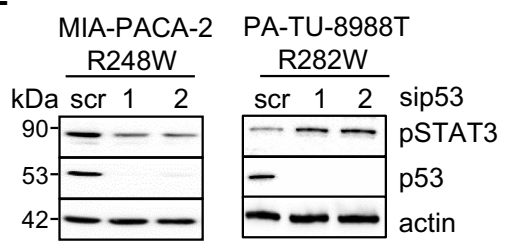

C
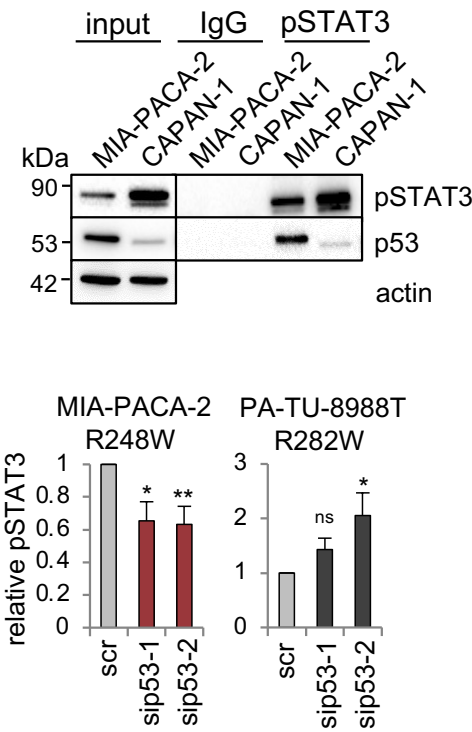

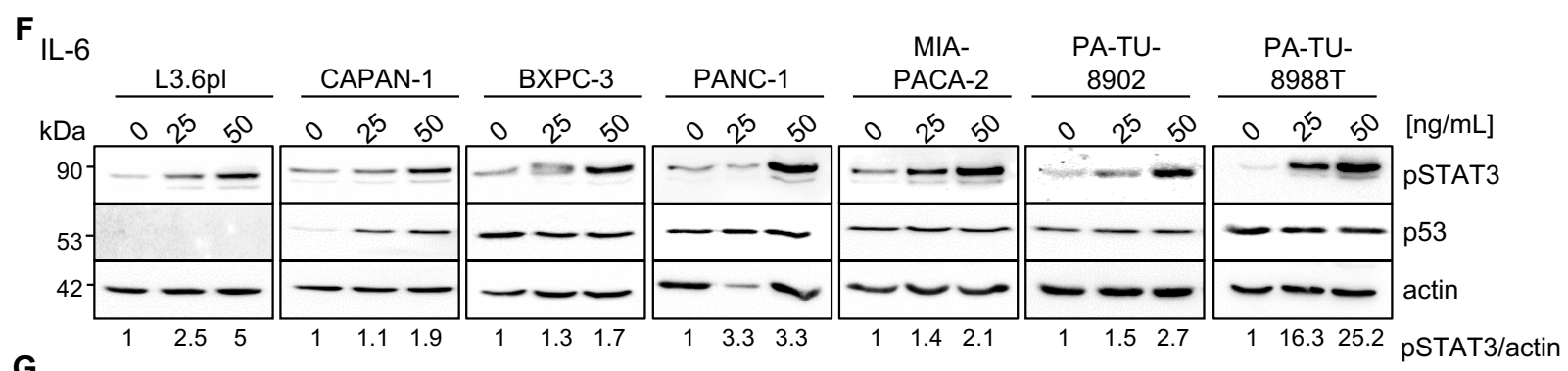

G

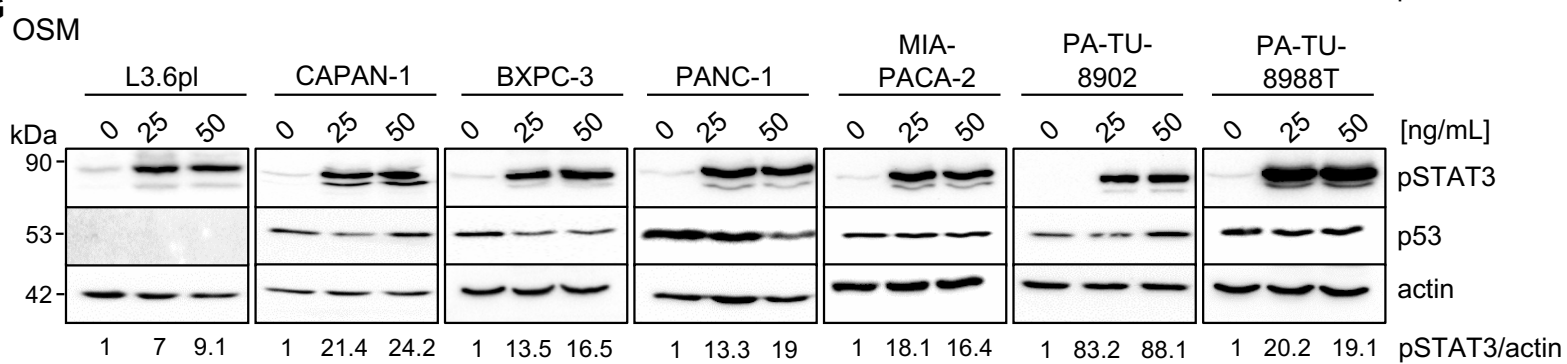

H

$\frac{\text { con }}{\text { input }} \stackrel{\lg G}{\text { pSTAT3 }} \frac{\mathrm{IL}-6}{\text { input }} \stackrel{\lg G}{\text { pSTAT3 }} \frac{\text { OSM }}{\text { input }} \frac{\lg G}{\text { pSTAT3 }}$

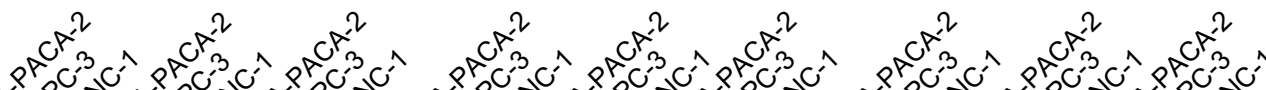

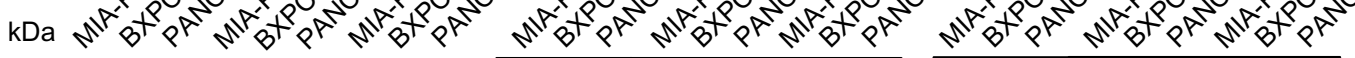

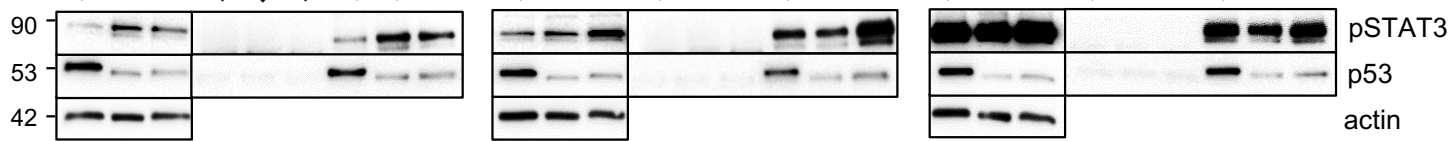

Figure 4 
Figure 4: Mutp53 ${ }^{\mathrm{R} 248 \mathrm{~W}}$ selectively binds to phosphorylated STAT3 in PDAC cells. (A) Representative immunoblot analysis of seven different PDAC cell lines. pSTAT3, pTyr 705STAT3 (Y705) and total STAT3 (tSTAT3). Actin, loading control. (B-D) Co-immunoprecipitations (ColPs) of untreated MIA-PACA-2, PANC-1, BXPC-3 (B), CAPAN-1 (C) and PA-TU-8988T (D) cells using anti-pSTAT3 (Y705) or IgG antibodies followed by immunoblot analysis. MIA-PACA-2 cells were always used as positive control. Note, the pSTAT3 band marked by an asterisk in (B) is an artefact due to a leaky pocket from the neighbouring MIA-PACA-2 lane. (E) Knockdown of mutp53 in MIA-PACA-2, but not in PA-TU-8988T cells downregulates pSTAT3 levels. Cells were transfected with two different siRNA against TP53 mRNA (sip53-1, -2) or scrambled control (scr) for $72 \mathrm{hrs}$ followed by immunoblot analysis. Representative immunoblot out of 3 (MIA-PACA-2) and out of 4 (PA-TU-8988T). Actin, loading control. (right) Diagrams represent the means \pm SEM of densitometric quantifications of three (MIA-PACA-2, $n=3$ ) or two (PA-TU-8988T, $n=4$ ) independent experiments, normalized to actin. Calculated relative to control scrambled siRNA (scr). Student's t test. ${ }^{*} p \leq 0.05 ;{ }^{* *} p \leq 0.01$; ns, not significant. $(F, G)$ Treatment of PDAC cell lines with the indicated concentrations of Interleukin-6 (IL-6, F), Oncostatin M (OSM, G) or respective solvent controls for $24 \mathrm{hrs}$. Representative immunoblot for pSTAT3 (Y705) induction is shown. Quantification by densitometry, normalized to actin loading control (pSTAT3/actin ratio) and calculated relative to solvent control. 'pSTAT3/actin', densitometric quantifications of the representative immunoblot, normalized to actin and relative to $0 \mathrm{ng} / \mathrm{ml} \mathrm{IL}-6$ or OSM treatments. (H) CoIPs of MIA-PACA-2, PANC-1 and BXPC-3 cells stimulated with $50 \mathrm{ng} / \mathrm{mL}$ IL-6, OSM or solvent control for 24 hrs. Immunoprecipitation using anti-pSTAT3 (Y705) or IgG antibodies, followed by immunoblot as indicated. Actin in unprecipitated input lysates, loading control. (B-D and $\mathrm{H}$ ) $5 \%$ of input were used for input control. 
A
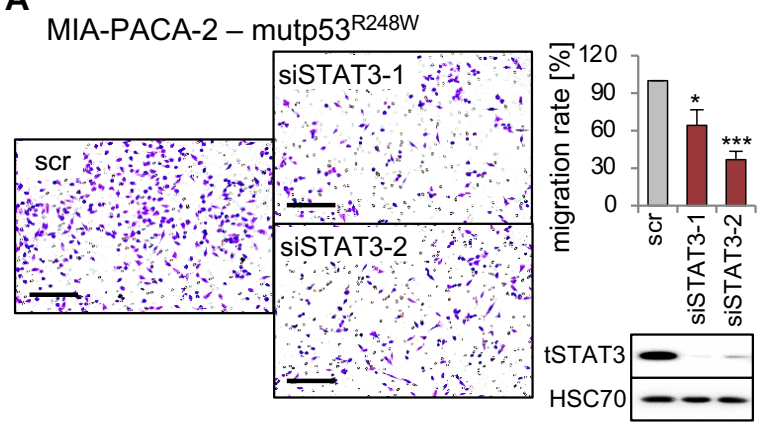

B

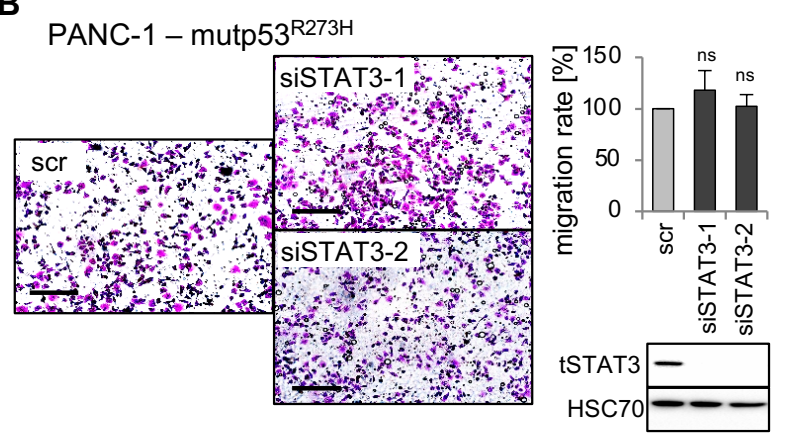

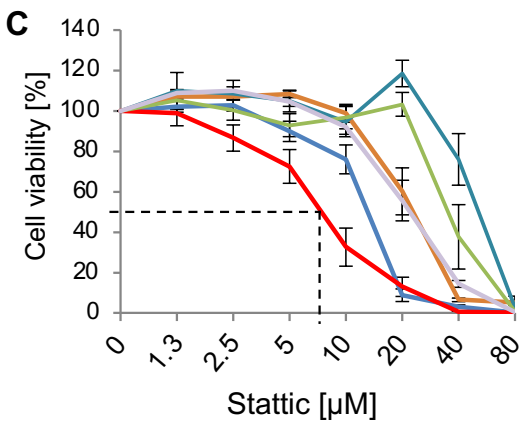

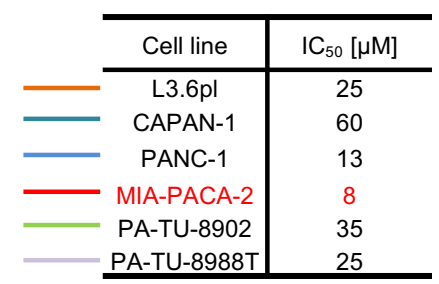

E

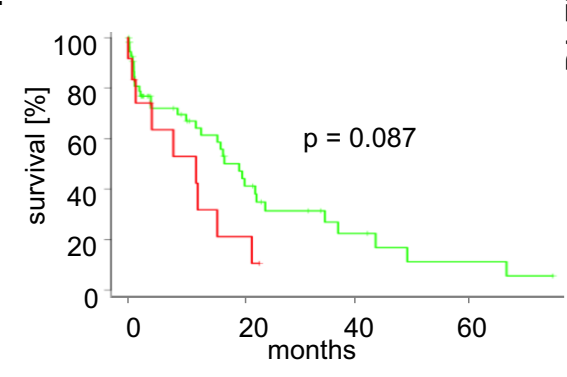

- TP53 ${ }^{\mathrm{R} 248}, \mathrm{n}=12$, median $12.0 \mathrm{mo}$

- TP53 NS/FS, n=55, median 19.7 mo
D
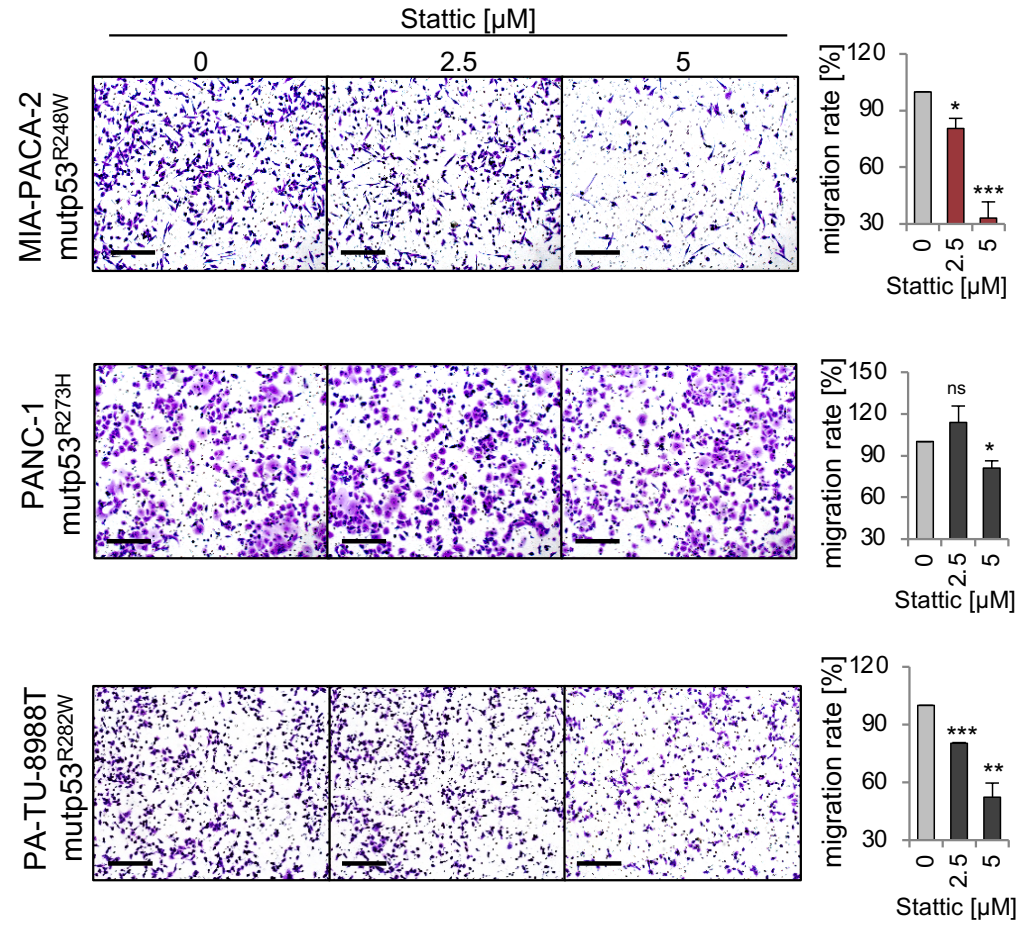

Figure 5 
Figure 5: $p 53^{\mathrm{R} 248 \mathrm{~W}}$ mutant selectively regulates STAT3 phosphorylation and activity in PDAC cells. (A, B) STAT3 knockdown phenocopies mutp53 knockdown in migration assays. MIA-PACA-2 (A) and PANC-1 (B) cells were transfected with two different siRNAs against STAT3 mRNA (siSTAT3-1, -2) or scrambled control (scr). $72 \mathrm{hrs}$ post-transfection cells were seeded into transwell inserts to assess their migration. After $24 \mathrm{hrs}$ cells were fixed, stained and counted at the membrane underside. Scale bars, $200 \mu \mathrm{m}$. MIA-PACA-2 cells: 4 biological replicates $(n=4)$, PANC-1 cells: 3 biological replicates, 2 out of 3 with 2 technical replicates $(n=5)$. Cells were calculated relative to scrambled control. Immunoblot analysis to confirm knockdown of STAT3. HSC70, loading control. (C) Cell viability assays of the indicated PDAC cell lines. Dose response curve after treatment with increasing concentrations of the STAT3 inhibitor Stattic or solvent control for $24 \mathrm{hrs}$. For each cell line 3-4 biological replicates were measured. Diagram represents means \pm SEM. From these curves $I_{50}$ values were determined, indicated in the table. Of note, MIA-PACA-2 cells are the most sensitive to Stattic treatment, indicated by the dashed line. (D) STAT3 inhibition phenocopies mutp53 knockdown in migration assays. Transwell migrations assays of MIA-PACA-2, PANC-1 and PA-TU-8988T cells treated with the indicated concentrations of Stattic for $24 \mathrm{hrs}$. Scale bars, $200 \mu \mathrm{m}$. For all cell lines, quantification of 2 biological replicates, 1 of them with 2 technical replicates ( $n=3$ total), calculated relative to $0 \mu M$ control treatment. (E) Survival curve of PDAC patients harboring TP53 R248 mutations versus patients harboring TP53 nonsense or frameshift (NS/FS) mutations. Number of patients and mean overall survival in months as indicated. TCGA data. Kaplan-Meier statistic, log-rank test. (A, B and D) Diagrams represent the means \pm SEM. Student's $t$ test. ${ }^{*} p \leq 0.05$; ${ }^{* *} p \leq 0.01$; ${ }^{* * *} p \leq 0.001$; ns, not significant. 


\section{Supplemental material}
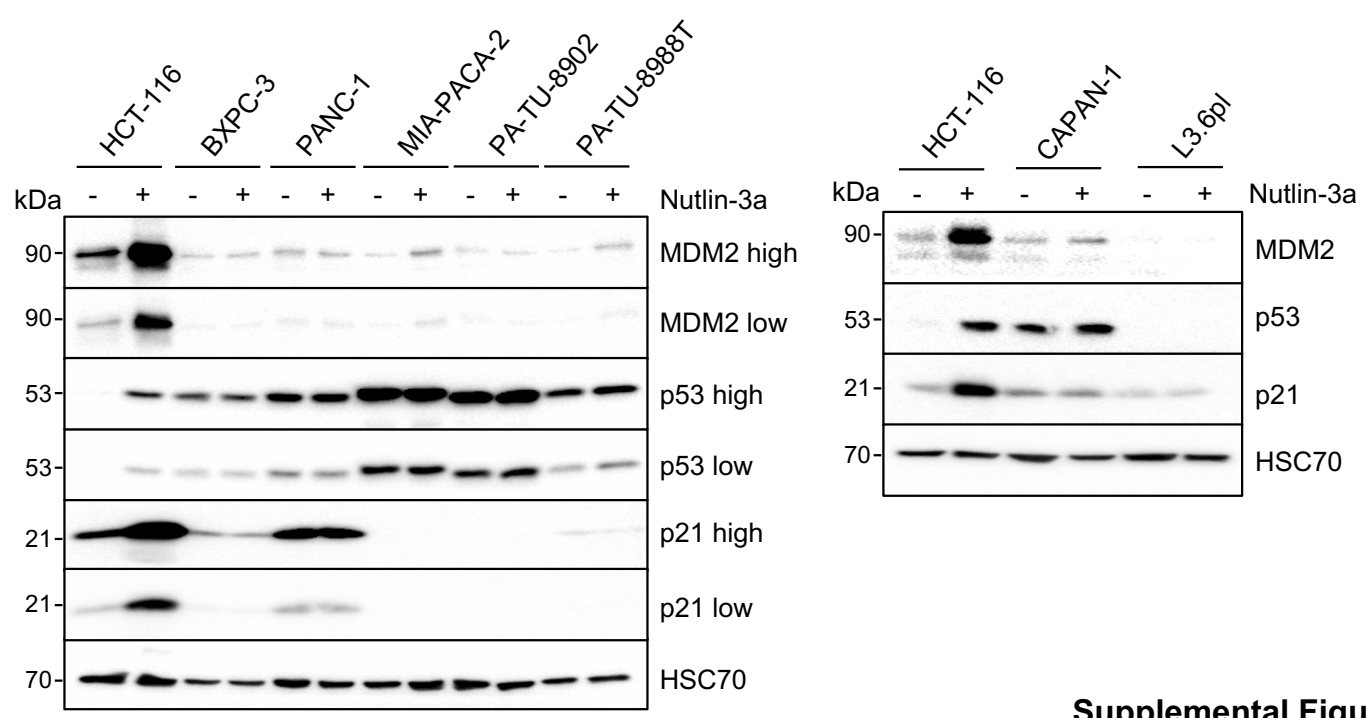

Supplemental Figure 1

Figure S1: Functional validation of missense p53 mutants in human PDAC cell lines. Representative immunoblots of seven PDAC cell lines harbouring various mutant p53 variants. Cells were treated with DMSO (-) or $10 \mu \mathrm{M}$ Nutlin-3a (+) for 8 hrs. Wildtype p53 containing HCT 116 cells served as functional positive control for p53 activation. 'high' and 'low' mean exposure time. CAPAN-1 (mutp53A159V), BXPC-3 (mutp53Y220C), PANC-1 (mutp53R273H), MIA-PACA2 (mutp53R248W), PA-TU-8902 (mutp53C176S) and PA-TU-8988T (mutp53R282W). L3.6pl cells harboring a truncating LOF mutation served as p53 null control. Note, all mutp53-containing cells failed to induce p53 targets p21 (CDKN1A, cyclin dependent kinase inhibitor 1A) and MDM2 (mouse double minute 2) after Nutlin-3a treatment. 
Klemke et al., manuscript provisionally accepted by Frontiers in Oncology (April 2021)

A

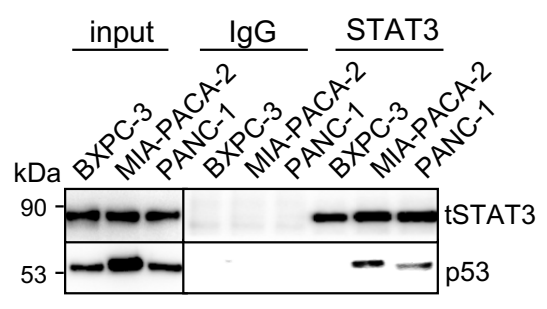

B

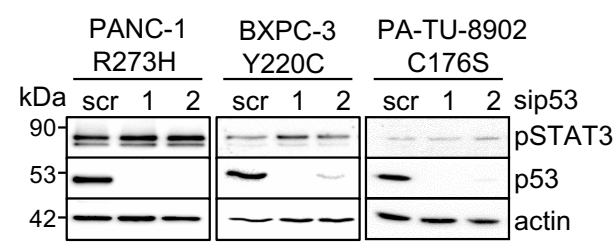

Supplemental Figure 2

Figure S2 related to Figure 4: Mutp53 ${ }^{\mathrm{R} 248 \mathrm{~W}}$ selectively binds to phosphorylated STAT3 in PDAC cells. (A) Co-immunoprecipitation (CoIP) of untreated BXPC-3, MIA-PACA-2 and PANC1 cells to investigate mutp53 binding to total STAT3 (tSTAT3). Immunoprecipitation with anti-total STAT3 (STAT3) or immunoglobulin G (IgG) antibodies, followed by immunoblot analysis. (B) Knockdown of mutp53 in PANC-1, BXPC-3 or PA-TU-8902 cells does not downregulate pSTAT3 levels. Cells were transfected with two different siRNA against TP53 mRNA (sip53-1, -2) or scrambled control (scr) for 72 hrs followed by immunoblot analysis. Representative immunoblots are shown. Actin, loading control. 
Klemke et al., manuscript provisionally accepted by Frontiers in Oncology (April 2021)

Supplemental material

Supplemental Table 1: Reagents and Resources

\begin{tabular}{|c|c|c|c|c|}
\hline REAGENT or RESOURCE & SOURCE & IDENTIFIER & & \\
\hline Antibodies & & & WB & $\begin{array}{l}\text { Co- } \\
\text { IP }\end{array}$ \\
\hline Rabbit monoclonal anti-Akt [D9E] & Cell Signaling & $\begin{array}{l}\text { 9272, } \\
\text { RRID:AB_329827 }\end{array}$ & $1: 1,000$ & \\
\hline Mouse polyclonal anti-beta-Actin & Abcam & $\begin{array}{l}\text { ab6276, } \\
\text { RRID:AB_2223210 }\end{array}$ & $1: 10,000$ & \\
\hline Rabbit polyclonal anti-beta-Actin & Abcam & $\begin{array}{l}\text { ab8227, } \\
\text { RRID:AB_2305186 }\end{array}$ & $1: 10,000$ & \\
\hline Mouse monoclonal anti-HSC70 [B-6] & Santa Cruz & $\begin{array}{l}\text { SC-7298, } \\
\text { RRID:AB } 627761\end{array}$ & $1: 5,000$ & \\
\hline $\begin{array}{l}\text { Mouse monoclonal anti-MDM2 (Ab-1) } \\
\text { [IF-2] }\end{array}$ & $\begin{array}{l}\text { Calbiochem } 囚 / \\
\text { Millipore }\end{array}$ & $\begin{array}{l}\text { OP46, } \\
\text { RRID:AB } 2335867\end{array}$ & $1: 300$ & \\
\hline $\begin{array}{l}\text { Rabbit monoclonal anti-p21 Waf1/Cip1 } \\
\text { [12D1] }\end{array}$ & Cell Signaling & $\begin{array}{l}2947, \\
\text { RRID:AB_823586 }\end{array}$ & $1: 1,000$ & \\
\hline Mouse monoclonal anti-p53 [DO-1] & Santa Cruz & $\begin{array}{l}\text { SC-126, } \\
\text { RRID:AB } 628082\end{array}$ & $1: 10,000$ & \\
\hline $\begin{array}{l}\text { Mouse monoclonal anti-p53 [DO-1], } \\
\text { HRP conjugated }\end{array}$ & Santa Cruz & $\begin{array}{l}\text { SC-126 HRP, } \\
\text { RRID:AB_628082 }\end{array}$ & $1: 1,000$ & $3 \mu \mathrm{g}$ \\
\hline $\begin{array}{l}\text { Rabbit monoclonal anti-phospho-Y705 } \\
\text { STAT3 [EP2147Y] }\end{array}$ & Abcam & $\begin{array}{l}\text { ab76315, } \\
\text { RRID:AB_1658549 }\end{array}$ & $1: 2,000$ & $3 \mu g$ \\
\hline Rabbit polyclonal anti-STAT3 & Santa Cruz & $\begin{array}{l}\text { SC-482, } \\
\text { RRID:AB_632440 }\end{array}$ & $1: 1,000$ & \\
\hline Rabbit monoclonal anti-STAT3 [79D7] & Cell Signaling & $\begin{array}{l}\text { 4904, } \\
\text { RRID:AB_331269 }\end{array}$ & $1: 1,000$ & $3 \mu \mathrm{g}$ \\
\hline Rabbit monoclonal anti-lgG [EPR25A] & Abcam & $\begin{array}{l}\text { ab172730, } \\
\text { RRID:AB_2687931 }\end{array}$ & & $3 \mu \mathrm{g}$ \\
\hline goat anti-rabbit IgG-HRP & Santa Cruz & $\begin{array}{l}\text { SC-2004, } \\
\text { RRID:AB_631746 }\end{array}$ & $1: 10,000$ & \\
\hline goat anti-mouse IgG-HRP & Santa Cruz & $\begin{array}{l}\text { SC-2005, } \\
\text { RRID:AB_631736 }\end{array}$ & $1: 10,000$ & \\
\hline \multicolumn{5}{|c|}{ Chemicals, Peptides and Recombinant Proteins } \\
\hline BCA protein assay & Pierce & \multicolumn{3}{|l|}{23227} \\
\hline $\begin{array}{l}\text { CellTiter-Glo } ® \text { Luminescent Cell Viability } \\
\text { Assay }\end{array}$ & Promega & \multicolumn{3}{|l|}{ G7571 } \\
\hline Clarity Max ${ }^{\mathrm{TM}}$ Western ECL Substrate & BioRad & \multicolumn{3}{|l|}{1705062} \\
\hline $\begin{array}{l}\text { cOmplete }{ }^{\mathrm{TM}} \text { mini protease inhibitor } \\
\text { cocktail }\end{array}$ & Roche & \multicolumn{3}{|l|}{11836170001} \\
\hline Crystal violet (C.I. 42555) & Roth & \multicolumn{3}{|l|}{$\mathrm{T} 123.1$} \\
\hline Cycloheximide & Sigma-Aldrich & \multicolumn{3}{|l|}{ C7698 } \\
\hline $\begin{array}{l}\text { Dimethyl sulfoxide (DMSO) Cell culture } \\
\text { grade }\end{array}$ & AppliChem & \multicolumn{3}{|l|}{ A3672 } \\
\hline EDTA & Roth & \multicolumn{3}{|l|}{8040.1} \\
\hline Ethanol absolut & $\begin{array}{l}\text { Chemsolute } ₫ / \\
\text { Th.Geyer }\end{array}$ & \multicolumn{3}{|l|}{2246} \\
\hline Ganetespib & $\begin{array}{l}\text { Provided by } \\
\text { Synta } \\
\text { Pharmaceuticals }\end{array}$ & \multicolumn{3}{|l|}{$N / A$} \\
\hline Imidazol & Roth & \multicolumn{3}{|l|}{899.2} \\
\hline $\begin{array}{l}\text { Immobilion Western chemiluminescent } \\
\text { HRP substrate }\end{array}$ & Millipore/Merck & \multicolumn{3}{|l|}{ WBKLS0500 } \\
\hline
\end{tabular}


Klemke et al., manuscript provisionally accepted by Frontiers in Oncology (April 2021)

Supplemental material

\begin{tabular}{|c|c|c|c|c|c|c|c|}
\hline \multicolumn{3}{|c|}{ InSolution'M MG-132 } & \multicolumn{2}{|c|}{$\begin{array}{l}\text { Calbiochem®/ } \\
\text { Merck }\end{array}$} & \multicolumn{3}{|l|}{474791} \\
\hline \multicolumn{3}{|l|}{ Interleukin-6 } & \multicolumn{2}{|c|}{ Immunotools } & \multicolumn{3}{|l|}{11340064} \\
\hline \multicolumn{3}{|l|}{ Methanol } & \multicolumn{2}{|l|}{ Roth } & \multicolumn{3}{|l|}{8388.3} \\
\hline \multicolumn{3}{|l|}{ Milk powder } & \multicolumn{2}{|l|}{ Roth } & \multicolumn{3}{|l|}{ T145.4 } \\
\hline \multicolumn{3}{|l|}{$\mathrm{NaCl}$} & \multicolumn{2}{|l|}{ Roth } & \multicolumn{3}{|l|}{3957.2} \\
\hline \multicolumn{3}{|l|}{$\mathrm{NaF}$} & \multicolumn{2}{|c|}{ AppliChem } & \multicolumn{3}{|l|}{ A0401 } \\
\hline \multicolumn{3}{|c|}{ Nitrocellulose membranes } & \multicolumn{2}{|c|}{ Amersham } & \multicolumn{3}{|l|}{ GE10600001 } \\
\hline \multicolumn{3}{|c|}{ Nonidet $^{\text {TM }}$ P 40 Substitute } & \multicolumn{2}{|c|}{ Sigma-Aldrich } & \multicolumn{3}{|l|}{74385} \\
\hline \multicolumn{3}{|c|}{ Nutlin-3a } & \multicolumn{2}{|c|}{ BOC Sciences } & \multicolumn{3}{|l|}{ B0084-425358 } \\
\hline Onalespib & & & Selleckch & & S1163 & & \\
\hline Oncostatin M & & & Immunotc & ols & 11344223 & & \\
\hline Protein G Sep & 4 Fast Flow & & GE Healt & care & 17061805 & & \\
\hline SDS & & & Roth & & CN30.3 & & \\
\hline Sodium deoxy & & & Sigma-Al & Irich & 30970 & & \\
\hline Sodium orthov & & & Sigma-Al & Irich & S6508 & & \\
\hline Stattic & & & Santa $\mathrm{Cr}$ & & sc-202818 & & \\
\hline $\begin{array}{l}\text { SuperSignal }{ }^{\mathrm{TM}} \\
\text { Sensitivity Suk }\end{array}$ & Femto Maxin & ium & \begin{tabular}{|l} 
ThermoF \\
Scientific \\
\end{tabular} & sher & 34095 & & \\
\hline Tris-HCl & & & Roth & & 4855.3 & & \\
\hline TritonX-100 & & & AppliChe & & A1388 & & \\
\hline Tween-20 & & & AppliChe & & A4974 & & \\
\hline Reagents for & ulture & & & & & & \\
\hline DMEM & & & Gibco & & 31600091 & & \\
\hline FBS & & & Merck & & S0615 & & \\
\hline L-Glutamine & & & Gibco & & 25030123 & & \\
\hline Mycoplasma [ & on Kit & & Lonza & & LT07-318 & & \\
\hline Penicillin-Stre & & & Gibco & & 15140122 & & \\
\hline RPMI 1640 & & & Gibco & & 42401042 & & \\
\hline $\begin{array}{l}\text { Lipofectamine } \\
\text { Reagent }\end{array}$ & 0 Transfectio & & Invitroger & & L3000015 & & \\
\hline $\begin{array}{l}\text { Lipofectamine } \\
\text { Reagent }\end{array}$ & 0 Transfectio & & Invitroger & & 11668019 & & \\
\hline TC treated $6 \mathrm{v}$ & & & Sarstedt & & 83.3920 & & \\
\hline TC treated 96 & ates & & Corning & & 3903 & & \\
\hline $\begin{array}{l}\text { Falcon }^{\circledR} \text { Perm } \\
\text { Plate with } 8.0 \\
\text { Membrane }\end{array}$ & $\begin{array}{l}\text { Support for } 22 \\
\text { ansparent PE }\end{array}$ & $\begin{array}{l}- \text {-well } \\
T\end{array}$ & Corning & & 353097 & & \\
\hline $\begin{array}{l}\text { Falcon }^{\circledR} 24-w \epsilon \\
\text { Polystyrene P } \\
\text { Companion PI }\end{array}$ & $\begin{array}{l}\text { reated Cell } \\
\text { le Support }\end{array}$ & & Corning & & 353504 & & \\
\hline Experimental & Is: Cell lines & & & & & & \\
\hline & & & & 96 well & $\begin{array}{c}\text { Transwell } \\
\text { insert }\end{array}$ & $\begin{array}{l}6 \text { well } \\
\mathrm{cmp}\end{array}$ & $\begin{array}{c}6 \text { well } \\
\text { transfection }\end{array}$ \\
\hline L3.6pl & $\begin{array}{l}\text { RRID:CV } \\
\text { CL_0384 }\end{array}$ & $\begin{array}{l}\text { PMI } \\
239 \\
\text { PMI } \\
109\end{array}$ & $\begin{array}{l}23 ; \\
70\end{array}$ & 5,000 & - & 150,000 & - \\
\hline MIA-PACA-2 & $\begin{array}{l}\text { DSMZ, } \\
\text { RRID:CV } \\
\text { CL_0428 }\end{array}$ & $\mathrm{ACC}$ & & 3,000 & 70,000 & 120,000 & 80,000 \\
\hline
\end{tabular}


Klemke et al., manuscript provisionally accepted by Frontiers in Oncology (April 2021)

Supplemental material

\begin{tabular}{|c|c|c|c|c|c|c|c|}
\hline PANC-1 & $\begin{array}{l}\text { ATCC, } \\
\text { RRID:CV } \\
\text { CL_0480 }\end{array}$ & \multicolumn{2}{|c|}{ CRL-1469 } & 5,000 & 50,000 & 200,000 & 100,000 \\
\hline PA-TU-8988T & $\begin{array}{l}\text { DSMZ, } \\
\text { RRID:CV } \\
\text { CL 1847 }\end{array}$ & \multicolumn{2}{|c|}{ ACC 162} & 4,000 & 70,000 & 150,000 & 90,000 \\
\hline PA-TU-8902 & $\begin{array}{l}\text { DSMZ, } \\
\text { RRID:CV } \\
\text { CL_1845 }\end{array}$ & \multicolumn{2}{|c|}{ ACC 179} & 4,000 & 70,000 & 150,000 & 100,000 \\
\hline BXPC-3 & $\begin{array}{l}\text { ATCC, } \\
\text { RRID:CV } \\
\text { CL_0186 }\end{array}$ & \multicolumn{2}{|c|}{ CRL-1687 } & 5,000 & 100,000 & 200,000 & 100,000 \\
\hline CAPAN-1 & $\begin{array}{l}\text { ATCC, } \\
\text { RRID:CV } \\
\text { CL } 0237\end{array}$ & \multicolumn{2}{|c|}{ HTB-79 } & 10,000 & - & 300,000 & - \\
\hline & & & & & & $\mathrm{cmp}, \mathrm{comp}$ & \\
\hline \multicolumn{8}{|c|}{ Oligonucleotides and Recombinant DNA } \\
\hline \multicolumn{3}{|c|}{$\begin{array}{l}\text { siRNA Silencer TM Select Negative } \\
\text { Control No. } 2 \text { siRNA (scr) }\end{array}$} & \multicolumn{2}{|c|}{ Invitrogen } & \multicolumn{3}{|l|}{4390847} \\
\hline \multicolumn{3}{|c|}{ siRNA p53 Silencer TM Select No.1 } & \multicolumn{2}{|c|}{$\begin{array}{l}\text { ThermoFisher } \\
\text { Scientific }\end{array}$} & \multicolumn{3}{|c|}{ 4390824, siRNA ID s605 } \\
\hline \multicolumn{3}{|c|}{ siRNA p53 Silencer ${ }^{\mathrm{TM}}$ Select No.2 } & \multicolumn{2}{|c|}{$\begin{array}{l}\text { ThermoFisher } \\
\text { Scientific }\end{array}$} & \multicolumn{3}{|c|}{ 4390824, siRNA ID s606 } \\
\hline \multicolumn{3}{|c|}{ siRNA p53 Silencer ${ }^{\mathrm{TM}}$ Select No.3 } & \multicolumn{2}{|c|}{$\begin{array}{l}\text { ThermoFisher } \\
\text { Scientific }\end{array}$} & \multicolumn{3}{|c|}{ AM51331, siRNA ID 106141} \\
\hline \multicolumn{3}{|c|}{ siRNA STAT3 Silencer ${ }^{\text {TM }}$ Select No.1 } & \multicolumn{2}{|c|}{$\begin{array}{l}\text { ThermoFisher } \\
\text { Scientific }\end{array}$} & \multicolumn{3}{|c|}{ 4390824, siRNA ID s743 } \\
\hline \multicolumn{3}{|c|}{ siRNA STAT3 Silencer'TM Select No.2 } & \multicolumn{2}{|c|}{$\begin{array}{l}\text { ThermoFisher } \\
\text { Scientific }\end{array}$} & \multicolumn{3}{|c|}{ 4390824, siRNA ID s744 } \\
\hline \multicolumn{8}{|c|}{ Software and Algorithms } \\
\hline \multicolumn{3}{|c|}{ ImageJ software } & \multicolumn{2}{|c|}{ Open source } & \multicolumn{3}{|c|}{ https://imagej.net/Welcome } \\
\hline \multicolumn{3}{|c|}{ Image Lab ${ }^{\mathrm{TM}}$ Software } & \multicolumn{2}{|c|}{ Biorad } & $\begin{array}{l}\text { http://www.bi } \\
\text { de/product/im }\end{array}$ & $\begin{array}{l}\mathrm{rad} . c o m / \mathrm{de} \\
\text { ge-lab-soft }\end{array}$ & \\
\hline ZEN & & & Zeis & & $\begin{array}{l}\text { https://www.z } \\
\text { mikroskopsof }\end{array}$ & $\begin{array}{l}\text { ss.de/mikr } \\
\text { are/zen.ht }\end{array}$ & pie/produkte/ \\
\hline Image Lab ${ }^{\mathrm{TM}} \mathrm{S}$ & & & Bior & & $\begin{array}{l}\text { http://www.bi } \\
\text { de/product/im }\end{array}$ & $\begin{array}{l}\text { rad.com/de } \\
\text { ge-lab-soft }\end{array}$ & \\
\hline Adobe Photosh & ftware & & Ado & & $\begin{array}{l}\text { https://www.a } \\
\text { ans.html }\end{array}$ & be.com/d & ativecloud/pl \\
\hline Celigo Imaging & neter & & $\begin{array}{l}\text { Nex } \\
\text { Bios }\end{array}$ & & $\begin{array}{l}\text { https://www.n } \\
\text { products/cellc } \\
\text { cytometers/ce }\end{array}$ & $\begin{array}{l}\text { xcelom.con } \\
\text { leter-and-c } \\
\text { go-imaging }\end{array}$ & $\begin{array}{l}\text { xcelom- } \\
\text { o-image- } \\
\text { ometer/ }\end{array}$ \\
\hline
\end{tabular}




\section{Supplemental cell lines authentication}

Leibniz-Institut

DSMZ-Deutsche Sammlung von

Mikroorganismen und Zellkulturen $\mathrm{GmbH}$

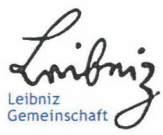

Georg-August-Universität Göttingen

Frau Luisa Klemke

Molekulare Onkologie

Justus-von-Liebig-Weg 11

37077 Göttingen

Ihr Zeichen/Your ref.

Dear Ms. Klemke,

Thank you for your request for authentication of human cell lines and species verification of animal cell line samples. We performed DNA profiling using 17 different and highly polymorphic STR (Short Tandem Repeat) loci. In addition, we have tested your human samples for the presence of mitochondrial DNA sequences from rodent cells such as mouse, rat, Chinese and Syrian hamster.

Animal cell line samples have been subjected to the procedure of Cytochrome C Subunit I (COI) DNA Barcoding for identification of the species.

Results (table 1):

\begin{tabular}{|c|c|c|c|}
\hline & sample & parental/reference line & comment/match \\
\hline 1 & L3.6pl & Colo-357 (Kajiji SM et al., 1987) & full-matching STR profile of cell line COLO-357 in the reference database, authentic* \\
\hline 2 & MIA-PACA-2 & MIA-PACA-2 (DSMZ ACC 733) & full-matching STR profile of cell line MIA-PACA- 2 in the reference database, authentic* \\
\hline 3 & PANC-1 & PANC-1 (DSMZ ACC 783) & full-matching STR profile of cell line PANC-1 in the reference database, authentic \\
\hline 4 & BXPC-3 & BXPC-3 (DSMZ ACC 760) & full-matching STR profile of cell line BXPC-3 in the reference database, authentic. \\
\hline 5 & CAPAN-1 & CAPAN-1 (DSMZ ACC 244) & full-matching STR profile of cell line CAPAN-1 in the reference database, authentic \\
\hline 6 & PA-TU-8902 & PA-TU-8902 (DSMZ ACC 179) & full-matching STR profile of cell line PA-TU-8902 the reference database, authentic \\
\hline 7 & HCT116 WT & HCT-116 WT (DSMZ ACC 581) & full-matching STR profile of cell line HCT-116 in the reference database, authentic* \\
\hline 8 & RKO & RKO (ATCC CRL-2577) & full-matching STR profile of cell line RKO in the reference database, authentic* \\
\hline 9 & LS174.T & LS174.T (DSMZ ACC 759) & full-matching STR profile of cell line LS174.T in the reference database, authentic* \\
\hline 10 & SW480 & SW-480 (DSMZ ACC 313) & full-matching STR profile of cell line SW-480 in the reference database, authentic \\
\hline 11 & SW837 & SW-837 (ATCC CRL-235) & fullmatching STR profile of cell line SW-837 in the reference database, authentic \\
\hline 12 & SW620 & SW-620 (ATCC CRL-227) & matching STR profile of cell line SW- 620 in the reference database, authentic \\
\hline 13 & DLD-1 & DLD-1 (DSMZ ACC 278) & full-matching STR profile of cell line DLD-1 in the reference database, authentic \\
\hline 14 & HT-29 & HT-29 (DSMZ ACC 299) & full-matching STR profile of cell line HT-29 in the reference database, authentic \\
\hline 15 & H1299 & H1299 (ATCC CRL-5803) & full-matching STR profile of cell line $\mathrm{H}-1299$ in the reference database, authentic* \\
\hline 16 & SJSA & SJSA (ATCC CRL-2098) & full-matching STR profile of cell line SJSA in the reference database, authentic \\
\hline 17 & Vero E6 & Vero E6 (ATCC CRL-1033) & COI DNA Barcoding analysis revealed Chlorocebus aethiops species, species-specific \\
\hline 18 & Calu-3 DPZ & Calu-3 DPZ (ATCC HTB-055) & full-matching STR profile of cell line CALU-3in the reference database, authentic \\
\hline 19 & Calu-3 ATCC & Calu-3 ATCC (ATCC HTB-055) & full-matching STR profile of cell line CALU-3 in the reference database, authentic \\
\hline 20 & RPE WT & hTERT-RPE (ATCC CRL-4000) & full-matching STR profile of cell line hTERT-RPE in the reference database, authentic \\
\hline 21 & HCC1806 & HCC1806 (ATCC CRL-2335) & full-matching STR profile of cell line $\mathrm{HCC} 1806$ in the reference database, authentic \\
\hline
\end{tabular}

Geschäftsführung/Directors:

Prof. Dr. Jörg Overman

Bettina Fischer

Aufsichtsratsvorsitzender/Head of

Supervisory Board: MR Dr. David Schnieders
Braunschweigische Landessparkasse

(NORD/LB) Kto.-Nr./Account: 203922

BLZ/Bank Code: 25050000

IBAN DE22 250500000002039220

SWIFT (BIC) NOLADE $2 \mathrm{H}$
Handelsregister/

Commercial Register:

Amtsgericht Braunschweig

HRB 2570

Steuer-Nr. 13/200/2403

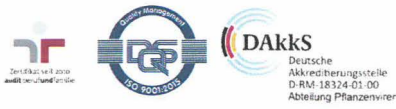


The proof for rodent cells in all samples was negative: At a detection limit of $1: 10^{-5}$ we could not detect mitochondrial sequences of Mus musculus, Rattus norvegicus, Cricetulus auratus, and Cricetulus griseus. Vice versa, sample 17 Vero E6 of Chlorocebus aethiops species is additionally free of human cells at a detection limit of $10^{-3}$.

We have carried out a search of the generated STR profiles of your samples which showed predominantly full matches with the STR data sets of respective parental human cell lines. With the exception of animal sample 17, the human samples are of authentic origin as highlighted in green in table 1.

*Asteriks-marked samples of table 1 reveal either the phenomenon of Microsatellite Instability (MSI) or Loss of Heterozygosity (LoH) at STR loci, which are marked in green color in table 2 (allelic list enclosed). MSI and LoH are often observed in cell lines after bottlenecking selection procedures (e. g. immortalization, gene transfer experiments etc.) in combination with loss of DNA Mismatch Repair. As a consequence respective drifted or lost alleles can become visible without any impact on the status of authenticity. To ensure the STR results we have performed VNTR Typing which could confirm the predicted identities and are free of additional costs (gel documentation in excel sheet).

For authentication of animal cell line samples we have carried out DNA Barcoding by PCR amplification of the $5^{\prime}$ coding region of Cytochrome $C$ Oxidase Subunit I and sequencing of the respective PCR product. Alignment of COI sequence revealed that sample Vero-E6 represents a cell culture of Chlorocebus aethiops (Green Monkey) indicating the correct species of primate cell line (table 1 ).

A further individualization of animal cell lines can often not be carried out because of the lack of suitable STR typing systems or is not possible in the case of rodent cells because of a lack of genetic variability by inbreeding.

The exclusion rate of the applied human STR system is indicating authenticity/uniqueness without any doubt, the matching probability of the system ranges from 1 in 1,114,000,000 for Caucasian and American.

Please find enclosed the documentation (STR electropherograms) and the allelic lists (tables 2 and 3).

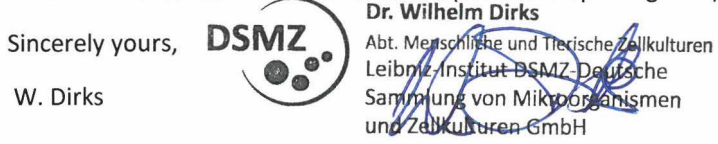

References for cell line subclone In.g.gffenstraße 7B • 38124 Braunschweig • Germany

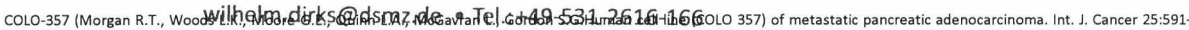
$598,1980)$

COLO357/FG (Kajiji S.M., Davceva B., Quaranta V. Six monoclonal antibodies to human pancreatic cancer antigens. Cancer Res. 47:1367-1376, 1987)

L3.1 (Vezeridis M.P., Tzanakakis G.N., Meitner P.A., Doremus C.M., Tibbetts L.M., Calabresi P. In vivo selection of a highly metastatic cell line from a human pancreatic carcinoma in

the nude mouse. Cancer 69:2060-2063, 1992)

L3.2 (Vezeridis M.P., Tzanakakis G.N., Meitner P.A., Doremus C.M., Tibbetts L.M., Calabresi P. In vivo selection of a highly metastatic cell line from a human pancreatic carcinoma in

the nude mouse. Cancer 69:2060-2063, 1992)

L3.3 (Vezeridis M.P., Tzanakakis G.N., Meitner P.A., Doremus C.M., Tibbetts L.M., Calabresi P. In vivo selection of a highly metastatic cell line from a human pancreatic carcinoma in the nude mouse. Cancer 69:2060-2063, 1992)

L3.6pl (Bruns C.J., Harbison M.T., Kuniyasu H., Eue I., Fidler I.J. In vivo selection and characterization of metastatic variants from human pancreatic adenocarcinoma by using orthotopic implantation in nude mice. Neoplasia 1:50-62, 1999)

Bettina Fischer

Aufsichtsratsvorsitzender/Head of

Supervisory Board: MR Dr. David Schnieders
Braunschweigische Landessparkasse (NORD/LB) Kto.-Nr./Account: 2039220 BLZ/Bank Code: 25050000 IBAN DE22 250500000002039220 SWIFT (BIC) NOLADE $2 \mathrm{H}$
Handelsregister/ Commercial Register: Amtsgericht Braunschweig HRB 2570

Steuer-Nr. 13/200/24030

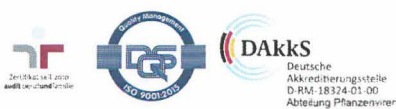


Klemke et al., manuscript provisionally accepted by Frontiers in Oncology (April 2021) Supplemental material

Lelbniz-Institut

DSMZ-Deutsche Sammlung von

Mikroorganismen und Zellkulturen $\mathrm{GmbH}$

Identification Service

Submission Form

Institute/Company

Name

Department

Street

Postal code and City

Country

E-mail

Telephone/Fax

(PO no. for your invoice)

Report language
Georg-August-Universität Göttingen

\section{Luisa Klemke}

Molekulare Onkologie

Justus-von-Liebig-Weg 11

37077 Göttingen

Germany

luisa.klemke@med.uni-goettingen.de

A-4501715941

English $X \quad$ German

$02 / 22 / 2021$

Date and signature

\begin{tabular}{|c|c|c|c|c|c|c|c|c|c|c|}
\hline & Sample Name & $\begin{array}{l}S \\
T \\
R\end{array}$ & $\begin{array}{l}c \\
0 \\
1\end{array}$ & \# & Sample Name & $\begin{array}{l}S \\
T \\
R\end{array}$ & $\begin{array}{l}\mathrm{c} \\
\mathrm{o}\end{array}$ & $\#$ & Sample Name & \begin{tabular}{|l}
5 \\
$T$ \\
\end{tabular} \\
\hline 1 & L3.6pl, 24.04.2018 & $x$ & & 8 & RKO, 29.10.2020 & $x$ & & 15 & H1299, 27.07.2020 & $x$ \\
\hline 2 & MIA-PACA-2, 13.11.2017 & $x$ & & 9 & LS174.T, 06.06.2018 & $x$ & & 16 & SJSA-1, 15.02.2021 & $\mathrm{x}$ \\
\hline 3 & PANC-1, 13.11.2017 & $x$ & & 10 & SW480, 28.08.2020 & $x$ & & 17 & Vero E6, 16.10.2020 & \\
\hline 4 & BXPC-3, 21.08.2017 & $x$ & & 11 & SW837, 13.01.2021 & $x$ & & 18 & Calu-3 DPZ, 08.12.2020 & $x$ \\
\hline 5 & CAPAN-1, 02.05.2018 & $x$ & & 12 & SW620, 24.06 .2020 & $x$ & & 19 & Calu-3 ATCC, 25.05 .2020 & $x$ \\
\hline 6 & U-8902, 28.01.2019 & $x$ & & 13 & DLD-1, 28.08.2020 & $x$ & & 20 & RPE htert WT, 10.05.2019 & \\
\hline 7 & HCT116 WT, 31.05 .2019 & $x$ & & 14 & HT-29, 06.08.2020 & $x$ & & 21 & HCC1806, 21.09.2020 & $x$ \\
\hline
\end{tabular}

Help

send e-mall

print form

Geschäftsfuhrung/Directors:

Prof. Dr. Lorg Overma

derithead of

Braunschwelgische landessparkasse (NORD/B) Kto.N. N./Account: 2039220 BLzeank Code: 25050000

02039220

Handeisregister/

Commercial Register:

Amtsgench Braunschweig

Steuer-Nr. 13/200/24030

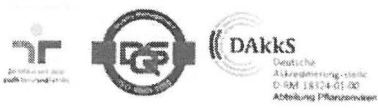


Leibniz-Institut

DSMZ-Deutsche Sammlung von

Mikroorganismen und Zellkulturen $\mathrm{GmbH}$

Leibniz-Institut

DSMZ-Deutsche Sammlung von

Mikroorganismen und Zellkulturen $\mathrm{GmbH}$

Inhoffenstrasse 7b

D-38124 Braunschweig

Germany

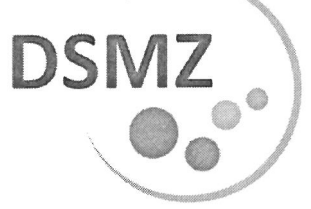

$\begin{array}{ll}\text { Date: } & 11.07 .2018 \\ \text { Your PO No.: } & \text { A - 4501481943 } \\ \text { Our order No.: } & \text { A1807330-1 } \\ \text { Contact: } & \text { Pia Palm }\end{array}$

Material Transfer Agreement

Between the DSMZ and the Recipient of Cell Line(s)

PLEASE RETURN A SIGNED COPY OF THIS AGREEMENT by email to mutz@dsmz.de

All material listed will be made available subject to the following conditions:

1. The DSMZ provides cells as a service to the research community. These materials are solely for research purposes and not for use in humans. Cell lines and their products shall not be sold or used for commercial purposes or utilized in any other type of commercial activity.

2. Recipients herewith confirm (also on behalf of their institutions) that they are end users of materials supplied by the DSMZ. Materials shall not be passed on to third parties.

3. Appropriate reference shall be made in any ensuing publication(s), crediting the work of the original investigators which established the cell line(s) and the DSMZ as supplier. No alteration may be made to their DSMZ titles or acronyms.

4. It is understood that neither the DSMZ nor the depositor of the cells accept any liability whatsoever in connection with the receipt, handling, storage or use of the cell line(s).

5. Recipients accept (also on behalf of host institutions) all conditions of ordering, delivery and payment of the DSMZ.

\begin{tabular}{|l|l|}
\hline ACC-No. & Name \\
\hline 162 & PA-TU-8988T \\
\hline
\end{tabular}

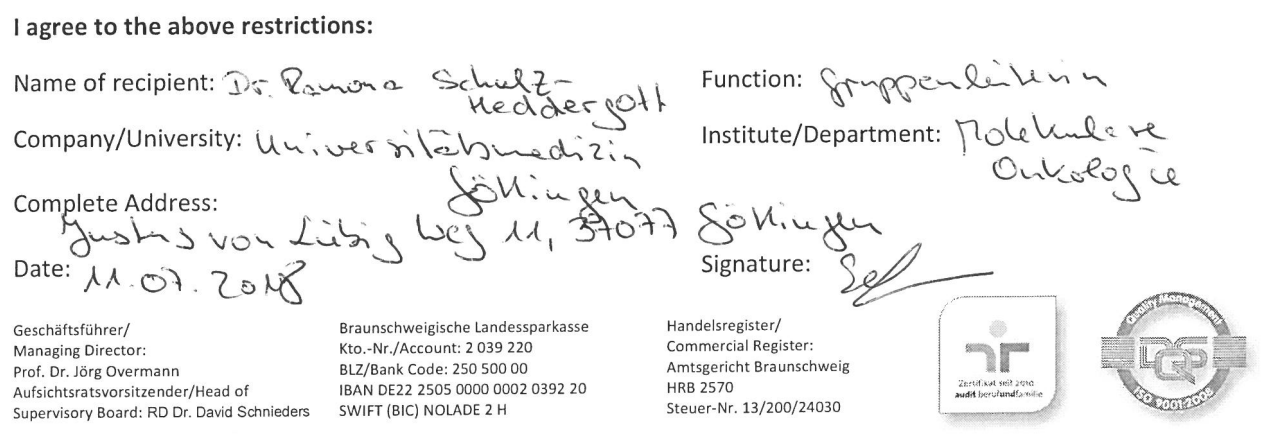


Klemke et al., manuscript provisionally accepted by Frontiers in Oncology (April 2021) Supplemental material

Leibniz-Institut

DSMZ-Deutsche Sammlung von

Mikroorganismen und Zellkulturen $\mathrm{GmbH}$

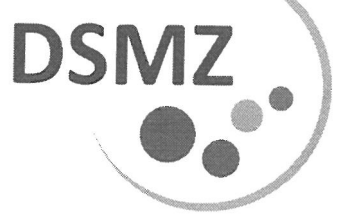

\begin{abstract}
Lieferadresse
Frau Magerhans

Justus von Liebig Weg 11

37077 GÖTTINGEN

DEUTSCHLAND

T.: 0551/39- 14128
\end{abstract}

Universitätsmedizin Göttingen

UMG - Molekulare Onkologie

Kundennr.: 30256

Rechnungsadresse

Georg-August-Universität Göttingen

Stiftung öffentlichen Recht

Universitätsmedizin

G3-1233 Kreditorenbuchhaltung

37099 GÖTTINGEN

DEUTSCHLAND

Kundennr.; 30419

$\begin{array}{ll}\text { Besteller: } & \text { Herr Julian Kloth } \\ \text { Bestellnr. : } & \mathrm{A}-4501481943 \\ \text { Bestelldatum.: } & 11.07 .2018 \\ \text { VAT Nr.: } & \\ \text { Rechnung vom: } & 18.07 .2018 \\ \text { Unsere Ref.-Nr.: } & \text { A } 1807330-1 \\ \text { Versand: } & 18.07 .2018 \\ \text { Ansprechpartner: } & \text { Pia Palm }\end{array}$

Lieferschein 01807236-1

\begin{tabular}{cccccc} 
Pos. & Menge & Leistung & Bezeichnung & Risikogruppe & Zolltarif-Nr. \\
\hline 1 & 1 & ACC 162 & PA-TU-8988T & 1 & 30029090
\end{tabular}

Bemerkungen:

Zollvermerk: Nettogewicht reine Biomasse: $0.9 \mathrm{~g}$

Sämtliche Lieferungen und Leistungen erfolgen auf Basis der Allgemeinen Geschäftsbedingungen der DSMZ (http://www.dsmz.de/terms.html)

Bitte beachten Sie unseren Begleitzettel.

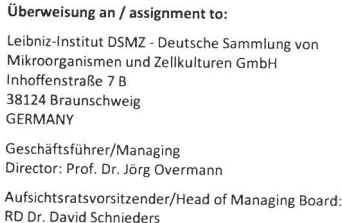

Kontakt / contact details: E-mail: contact@dsmz.de URL: http://www.dsmz.de Phone: +49(0) 53126160
Fax: $\quad+49(0) 5312616418$

St.-Nr.: $\quad 13 / 200 / 24030$

VAT-No.: DE 114815269

Handelsregister/Commercial Register Amtsgericht Braunschweig HRB 2570

DIN EN ISO 9001:2008

Bankverbindung / bank details: Braunschweigische Landessparkasse / Norddeutsche Landesbank Girozentrale (NORD/LB) Konto-Nr.: 2039220 (NAN (1) O. Box 3341,38023

8023 Braunschweig.
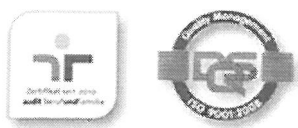


\section{Panel Genome Sequencing of human L3.6pl cells}

\begin{tabular}{|c|c|c|c|c|c|c|c|c|}
\hline Index Gene & Transcript & Type & Nuc Change & Coverage & Cha ( $H$ web Ref. & c. HGVS & p. I mu m TVî̀ $M$ & ' Weightin Filter Step \\
\hline 1 GNAS & ENST0000037108: E1 & $-37411[\mathrm{cC}$ & $G \rightarrow A$ (het) & $22 \%(356)[22 \%(171) / 22 \%(185)]$ & rs74934823 (10 & c. $-37835 \mathrm{G}>\mathrm{A}$ & & distinct \\
\hline 2 MAPK4 & ENST0000040038: E3 & -10553 [c C & $C \rightarrow T$ (homo) & $100 \%(705)[100 \%(442) / 100 \%(263)]$ & rs $7227230(100$ & c. $547-10553 C>T$ & & distinct \\
\hline 3 FAT4 & ENST0000039432: E2 & $-4905[\mathrm{ch} C$ & $\mathrm{T} \rightarrow \mathrm{C}$ (homo) & $100 \%(759)[100 \%(524) / 100 \%(235)]$ & rs925243(1000 & c.5176-4905T>C & & distinct \\
\hline 4 BRD2 & ENST0000044908: E1 & $-3818[\mathrm{ch} C$ & $\mathrm{G} \rightarrow \mathrm{A}$ (homo) & $99 \%(427)[100 \%(348) / 96 \%(79)]$ & rs116656352 (1 & c. $-3976 G>A$ & & distinct \\
\hline 5 BRD2 & ENST0000044908: E1 & $-3813[\mathrm{ch} C$ & $\mathrm{C} \rightarrow \mathrm{A}$ (homo) & $99 \%(466)[100 \%(379) / 97 \%(87)]$ & rs115634162 (1 & c. $-3971 C>A$ & & distinct \\
\hline 6 LMNA & ENST00000368301 E2 & -778 [chr: C & $C \rightarrow G($ homo $)$ & $100 \%(291)[99 \%(159) / 100 \%(132)]$ & rs665979 (1000 & c. $357-778 \mathrm{C}>\mathrm{G}$ & & distinct \\
\hline 7 LMNA & ENST0000036830। E2 & $-739[\mathrm{chr} C$ & $\mathrm{T}>\mathrm{G}$ (homo) & $100 \%$ (306) $[100 \%(137) / 100 \%(169)]$ & rs513043 (1000 & c.357-739T>G & $0 / 2$ of 4 & distinct \\
\hline 8 BRD2 & ENSTO000044908: E7 & -232 [chric & $A \rightarrow G($ homo $)$ & $99 \%(391)[100 \%(235) / 99 \%(156)]$ & rs3129307 (100 & c. $1060-232 A>G$ & & distinct \\
\hline $9 \mathrm{BRD} 2$ & ENST0000044908: E9 & $-53 \ldots-.51 / \vdots D$ & CTT (homo) & $93 \%(589)[95 \%(333) / 91 \%(256)]$ & & 38-53_1438-51delCT & & distinct \\
\hline 10 KMT2D & ENST0000030106 E32 & $-46[\mathrm{chr} 1 \mathrm{C}$ & $\mathrm{G} \rightarrow \mathrm{A}$ (het) & $33 \%(378)[32 \%(202) / 33 \%(176)]$ & & c. $8047-46 G>A$ & & distinct \\
\hline 11 LMNA & ENST0000036830। E7 & $-43[\mathrm{chr} 1 \mathrm{C}$ & $A \rightarrow G$ (het) & $64 \%(656)[68 \%(273) / 62 \%(383)]$ & rs16837198 (10 & c. $1158-43 A>G$ & & distinct \\
\hline 12 SLIT2 & ENST0000050415, E28 & $-42[\mathrm{chr} 4: \mathrm{C}$ & $A \rightarrow G$ (het) & $31 \%(267)[28 \%(104) / 33 \%(163)]$ & rs2290750 (100 & c. $2851-42 A>G$ & & distinct \\
\hline 13 SMARCA4 & ENST0000045071 E10 & $-37 . .-36 /$ indel & TG $\rightarrow$ CA (homo) & $92 \%(898)[94 \%(421) / 91 \%(477)]$ & rs386806808 (d7 & 52-37_1762-36delinsC & & distinct \\
\hline 14 MYCBP2 & ENST0000035733 E42 & $-20[\mathrm{chr} 1 \mathrm{C}$ & $A \rightarrow G($ homo $)$ & $100 \%(320)[100 \%(233) / 100 \%(87)]$ & rs3742103 (100 & c. $6001-20 \mathrm{~A}>\mathrm{G}$ & & distinct \\
\hline 15 SMARCA2 & ENST0000034972 E9 & -19 [chrg: C & $\mathrm{G} \rightarrow \mathrm{A}$ (het) & $36 \%(199) \quad[33 \%(103) / 39 \%(96)]$ & rs141618610 (1 & c. $1522-19 \mathrm{G}>\mathrm{A}$ & & istinct \\
\hline 16 LRP1B & ENST0000038948. E36 & $-17 / 1 \mathrm{bp}$ I (Dup) & $T$ (het) & $51 \%(207)[54 \%(168) / 43 \%(39)]$ & rs398104769 (d & c.5759-18dupT & & distinct \\
\hline 17 FGFR2 & ENST0000035848 E14 & -17 [chr11c & $T \rightarrow G$ (het) & $46 \%(203)[48 \%(143) / 44 \%(60)]$ & rs3135802 (100 & c.1864-17T>G & & distinct \\
\hline 18 LRP1B & ENST0000038948. E34 & $-17[\mathrm{chr} 2 \mathrm{C}$ & $A \rightarrow G$ (homo) & $100 \%(545)[100 \%(323) / 100 \%(222)]$ & rs7599219 (dbS & c. $5501-17 A>G$ & & istinct \\
\hline 19 TLE4 & ENST0000037655: E11 & -16 [chr9: C & C $\rightarrow \mathrm{T}$ (homo) & $100 \%(235)[100 \%(206) / 100 \%(29)]$ & rs73652238 (10 & c. $784-16 C>T$ & & istinct \\
\hline 20 MARK2 & ENST0000040201। E18 & $-16[\mathrm{chr} 1 \mathrm{C}$ & $C \rightarrow T$ (het) & $25 \%(258)[24 \%(132) / 25 \%(126)]]$ & & c.1962-16C>T & & istinct \\
\hline 21 КМт2C & ENST0000026218: E18 & $-15[\mathrm{chr} 7: \mathrm{C}$ & $\mathrm{T} \rightarrow \mathrm{C}$ (het) & $43 \%(178)[46 \%(120) / 39 \%(58)]$ & rs62481502 (db & c. $2872-15 T>C$ & & istinct \\
\hline 22 SF3B1 & ENST0000033550: E19 & $-15[\mathrm{chr} 2 \mathrm{C}$ & $A \rightarrow T$ (homo) & $100 \%(209)[100 \%(169) / 100 \%(40)]$ & rs788017 (1000 & c.2719-15A>T & & distinct \\
\hline 23 LRP1B & ENST0000038948, E45 & $-15[\mathrm{chr} 2 \mathrm{C}$ & $C \rightarrow G$ (het) & $57 \%(218)[61 \%(142) / 52 \%(76)]$ & rs11694934 (10 & c. $7388-15 C>G$ & & istinct \\
\hline $24 \mathrm{ROBO} 3$ & ENST0000039780: E3 & $-14[\mathrm{chr} 1 \mathrm{C}$ & $A \rightarrow C$ (het) & $69 \%(11)[100 \%(11) / 0 \%(0)]$ & rs11219820 (10 & c. $488-14 A>C$ & & orced, distinct \\
\hline 25 EGFR & ENST0000027549: E1 & $-14[\mathrm{chr} 7 \mathrm{C}$ & A $\rightarrow C$ (homo) & $100 \%(85)[100 \%(80) / 100 \%(5)]$ & rs712830(1000 & c. $-191 \mathrm{~A}>\mathrm{C}$ & & forced, distinct \\
\hline 26 BRD2 & ENST0000044908: E2 & $-13 / 1 \mathrm{bp}$ I (Dup) & $T$ (homo) & $93 \%(91)[93 \%(86) / 100 \%(5)]$ & & c.-112-14dupT & & forced, distinct \\
\hline 27 SLIT2 & ENST0000050415, E12 & $-12[\mathrm{chr} 4 \mathrm{i}$ C & $\mathrm{T} \rightarrow \mathrm{C}$ (homo) & $100 \%(235)[100 \%(154) / 100 \%(81)]$ & rs519813 (1000 & c. $1059-12 T>C$ & & istinct \\
\hline 28 SF3B1 & ENST0000033550: E13 & $-11 / 2 \mathrm{bp}$ I (Dup) & TT (homo) & $86 \%(166)[86 \%(129) / 86 \%(37)]$ & rs397804827 (d1) & 720-13_1720-12dup T & & distinct \\
\hline 29 RОВО2 & ENST0000048769. E2 & $-10[\mathrm{chr} 3: \mathrm{C}$ & $C \rightarrow G$ (het) & $79 \%(1972)[78 \%(1215) / 80 \%(757)]$ & rs9631539 (dbS & c. $-13-10 C>G$ & & distinct \\
\hline 30 BRD2 & ENST0000044908: E3 & $-10[\mathrm{chr} 6 \mathrm{C}$ & $\mathrm{T} \rightarrow \mathrm{C}$ (homo) & $99 \%(256)[99 \%(193) / 100 \%(63)]$ & rs635688(1000 & c. $193-10 T>C$ & & istinct \\
\hline 31 MYCBP2 & ENST0000035733 E13 & $-10 / 1 \mathrm{bp}$ I (Dup) & $T$ (het) & $55 \%(126)[69 \%(101) / 30 \%(25)]$ & rs549452634 (d & c.1534-11dupT & & istinct \\
\hline 32 KRAS & ENST0000025607: E6 & $-9[\mathrm{chr} 12 \mathrm{C}$ & $\mathrm{G} \rightarrow \mathrm{A}$ (het) & $37 \%(117)[38 \%(78) / 34 \%(39)]$ & rs12313763 (10 & c. $* 5-9 \mathrm{G}>\mathrm{A}$ & & istinct \\
\hline 33 SLIT2 & ENST0000050383 E9 & $-9[\mathrm{chr} 4: \mathrm{gC}$ & $G \rightarrow T$ (het) & $30 \%(191) \quad[30 \%(105) / 30 \%(86)]$ & rs7695303 (100 & c.776-9G>T & & istinct \\
\hline 34 KMT2D & ENST0000030106 E11 & $-7 / 1 \mathrm{bp}[\mathrm{D}$ & $\mathrm{C}$ (homo) & $96 \%(946)[96 \%(401) / 96 \%(545)]$ & rs112620957 (d & c. $2798-7 \mathrm{delC}$ & & istinct \\
\hline 35 BRAF & ENST0000028860: E1 & $-7[\mathrm{chr} 7: \mathrm{gC}$ & $A \rightarrow C$ (het) & $29 \%(7)[32 \%(7) / 0 \%(0)]$ & & c. $-68 \mathrm{~A}>\mathrm{C}$ & & forced, distinct \\
\hline 36 LRP1B & ENST0000038948, E27 & $-7[\mathrm{chr} 2: \mathrm{g} \mathrm{C}$ & $T \rightarrow G$ (het) & $51 \%(81)[53 \%(70) / 44 \%(11)]$ & rs12987572 (10 & c. $4335-7 \mathrm{~T}>\mathrm{G}$ & & distinct \\
\hline $37 \mathrm{NARF}$ & ENST0000030979، E9 & $-6[\mathrm{chr} 17: \mathrm{C}$ & $T>G$ (het) & $74 \%(704)[73 \%(336) / 76 \%(368)]]$ & rs12103494 (10 & c.834-6T>G & & istinct \\
\hline 38 ROBO1 & ENST0000046423: E22 & $-6 / 1 \mathrm{bp}[\mathrm{I}$ (Dup) & $T$ (het) & $83 \%(179)[88 \%(127) / 74 \%(52)]$ & rs369027577 (d & c.2883-7dupT & & istinct \\
\hline $39 \mathrm{APC}$ & ENST00000257431 E5 & $-4 / 1 \mathrm{bp}[\mathrm{D}$ & $A$ (het) & $74 \%(111)[82 \%(58) / 68 \%(53)]$ & COSM19338 (Cl & c.423-4delA & & istinct \\
\hline 40 BRD2 & ENST0000044908: E8 & $-4[\mathrm{chr} 6: \mathrm{g} C$ & $\mathrm{G} \rightarrow \mathrm{C}$ (homo) & $100 \%(981)[100 \%(597) / 100 \%(384)]$ & rs3097644(100 & c. $1189-4 G>C$ & & istinct \\
\hline 41 FAT1 & ENSTO000044180: E3 & $-4[\mathrm{chr} 4: \mathrm{gC}$ & $\mathrm{G} \rightarrow \mathrm{C}$ (homo) & $100 \%(354)[100 \%(259) / 100 \%(95)]$ & rs172903 (1000 & c. $3266-4 G>C$ & & distinct \\
\hline 42 PBRM1 & ENST00000394831 E11 & $-4[\mathrm{chr} 3: \mathrm{gC}$ & $G \rightarrow A$ (het) & $74 \%(287)[65 \%(104) / 81 \%(183)]$ & $\cos M 4002750$ & c.996-4G>A & & distinct \\
\hline 43 LRP1B & ENSTO000038948: E9 & $3(1239)$ [C & $\mathrm{T} \rightarrow \mathrm{C}$ (het) & $65 \%(77)[67 \%(74) / 38 \%(3)]$ & . V (413 rs1525579 (100 & c. $1239 \mathrm{~T}>\mathrm{C}$ & p.Val413= & forced, distinct \\
\hline 44 LRP1B & ENST0000038948. E50 & 5 (8031) ic & $A \rightarrow G$ (het) & $32 \%(122)[31 \%(73) / 33 \%(49)]$ & $Q(267$ COSM4001201 & c. $8031 \mathrm{~A}>\mathrm{G}$ & p. $G \ln 2677=$ & distinct \\
\hline 45 CDKN2A & ENST0000030449, E2 & $5(155)[\mathrm{cC}$ & $\mathrm{T} \rightarrow \mathrm{A}$ (homo) & $100 \%(932)[100 \%(567) / 100 \%(365)]$ & $>\mathrm{K}(52 ; \cos \mathrm{M} 13436(\mathrm{Cl}$ & c. $155 \mathrm{~T}>\mathrm{A}$ & p.Met52Lys & distinct \\
\hline 46 LRP1B & ENST0000038948. E54 & $5(8526) \mid C$ & $\mathrm{~T} \rightarrow \mathrm{C}$ (het) & $30 \%(123)[30 \%(78) / 30 \%(45)]$ & $\mathrm{Y}(2842$ COSM 3757652 & c. $8526 \mathrm{~T}>\mathrm{C}$ & p. $\operatorname{Tyr} 2842=$ & listinct \\
\hline $47 \mathrm{ROBO} 3$ & ENST0000039780 E21 & 5 (2991) ic & $\mathrm{G} \rightarrow \mathrm{A}$ (homo) & $100 \%(569)[100 \%(327) / 100 \%(242)]$ & -A $(997$ rs7933204 (100 & c. $2991 \mathrm{G}>\mathrm{A}$ & p.Ala997= & distinct \\
\hline 48 МYCBP2 & ENST0000035733 E55 & $8(7778)$ ic & A $\rightarrow G$ (homo) & $100 \%(542)[100 \%(316) / 100 \%(226)]$ & S (259: rs34474844 (10 & c. $7778 \mathrm{~A}>\mathrm{G}$ & p.Asn2593Ser & distinct \\
\hline 49 EGFR & ENST0000027549: E23 & $8(2709)$ ic & $\mathrm{T} \rightarrow \mathrm{C}$ (homo) & $100 \%$ (1254) $[100 \%(646) / 100 \%(608)]$ & . T (903 rs1140475 (100 & c. $2709 \mathrm{~T}>\mathrm{C}$ & p.Thr903= & distinct \\
\hline 50 SF3B1 & ENST0000033550: E5 & $8(423)[\mathrm{cC}$ & $A \rightarrow G($ homo $)$ & $100 \%$ (380) $[100 \%(163) / 100 \%(217)]$ & $\cdot \mathrm{K}(141$ COSM1129389 & c. $423 \mathrm{~A}>\mathrm{G}$ & p.Lys141= & listinct \\
\hline 51 POLR3A & ENST0000037237 E23 & $9(2997)$ |C & $G \rightarrow T$ (het) & $55 \%(377)[59 \%(190) / 52 \%(187)]$ & - V $(999$ rs12241228 (10 & c. $2997 \mathrm{G}>\mathrm{T}$ & p.Val999= & listinct \\
\hline 52 JAG1 & ENST0000025495: E20 & $10(2382) \mathrm{C}$ & $C \rightarrow T$ (het) & $62 \%(318)[64 \%(223) / 60 \%(95)]$ & . S (794) rs56225585(10 & c. $2382 C>T$ & p.Ser794= & distinct \\
\hline 53 FAT4 & ENST0000039432: E10 & $20(11814 ; \mathrm{C}$ & $A \rightarrow G$ (het) & $68 \%(262)[70 \%(180) / 65 \%(82)]$ & S (393\& rs17009721 (10 & c.11814A>G & p.Ser3938= & distinct \\
\hline 54 КМт2С & ENST0000026218: E7 & $22(871)$ |C & $C \rightarrow T$ (het) & $78 \%(538)[81 \%(387) / 71 \%(151)]$ & $\cdot F(291 ;$ rs56850341 (10 & $c .871 C>T$ & p.Leu291Phe & distinct \\
\hline 55 POLR3A & ENST0000037237 E16 & $26(2100) \mathrm{C}$ & $C \rightarrow T$ (het) & $67 \%(629)[67 \%(346) / 67 \%(283)]$ & . I (700) rs79793998 (10 & c. $2100 \mathrm{C}>\mathrm{T}$ & p.lle $700=$ & distinct \\
\hline 56 FAT1 & ENST0000044180: E11 & $26(8904) \mathrm{C}$ & $\mathrm{C} \rightarrow \mathrm{T}$ (homo) & $100 \%(277)[100 \%(226) / 100 \%(51)]$ & A (296\& rs1280099 (100 & c. $8904 \mathrm{C}>\mathrm{T}$ & p.Ala2968= & distinct \\
\hline 57 SLC25A24 & ENST0000056548: E1 & 27 [chr1:l C & $T>G$ (het) & $78 \%(101)[78 \%(100) / 100 \%(1)]$ & i' UTR rs662282(1000 & c. $-194 T>G$ & & forced, distinct \\
\hline 58 U2AF1 & ENSTO000029155: E1 & 29 [chr21C & $A \rightarrow C$ (het) & $49 \%(298)[48 \%(166) / 49 \%(132)]$ & i' UTR rs17115876(10 & c. $-65 \mathrm{~A}>\mathrm{C}$ & & distinct \\
\hline 59 МҮСВР2 & ENST0000035733' E62 & 29 (10440' C & $\mathrm{G} \rightarrow \mathrm{A}$ (homo) & $100 \%(236)[100 \%(139) / 100 \%(97)]$ & P (348C rs34700794 (10 & c. $10440 \mathrm{G}>\mathrm{A}$ & p.Pro3480= & distinct \\
\hline 60 FAT4 & ENST0000039432: E16 & $30(12846 ; \mathrm{C}$ & $C \rightarrow T$ (het) & $71 \%(280)[73 \%(187) / 67 \%(93)]$ & S (4282 rs17009819 (10 & c.12846C>T & p.Ser4282= & distinct \\
\hline $61 \mathrm{ROBO} 2$ & ENSTO000048769. E2 & 32 (19) [c C & $C \rightarrow A$ (het) & $79 \%(1720)[78 \%(832) / 80 \%(888)]$ & $\rightarrow S(7)$ rs12171318(db & c. $19 \mathrm{C}>\mathrm{A}$ & p.Arg7Ser & distinct \\
\hline $62 \mathrm{NARF}$ & ENST0000030979. E9 & $34(867)$ IC & $T->C$ (het) & $78 \%(726)[76 \%(307) / 80 \%(419)]$ & $\cdot R(289$ rs3829567 (100 & c. $867 \mathrm{~T}>\mathrm{C}$ & p.Arg289= & distinct \\
\hline 63 МYСВР2 & ENST0000035733' E46 & $37(6520) \mathrm{C}$ & $C \rightarrow T$ (homo) & $100 \%(329)[100 \%(220) / 100 \%(109)]$ & L (2174 rs34982494 (10 & c. $6520 \mathrm{C}>\mathrm{T}$ & p.Leu2174= & distinct \\
\hline 64 FGFR2 & ENST0000035848 E1 & $38[\mathrm{chr} 10 \mathrm{C}$ & $\mathrm{G} \rightarrow \mathrm{A}($ homo $)$ & $100 \%(275)[100 \%(80) / 100 \%(195)]$ & i' UTR rs1047111(100 & c. $-236 \mathrm{G}>\mathrm{A}$ & & distinct \\
\hline 65 SMARCC2 & ENST0000055016. E5 & $39(438)$ |C & A $\rightarrow G$ (homo) & $99 \%(785)[99 \%(318) / 100 \%(467)]$ & - $P(146$ rs7136420 (100 & c. $438 \mathrm{~A}>\mathrm{G}$ & p.Pro146= & distinct \\
\hline 66 BANF1 & ENSTO000031217: E1 & $39 / 2$ bp || (Dup) & AG (het) & $42 \%(291)[41 \%(165) / 44 \%(126)]$ & IUTR & c. -472 - 471 dupAG & & distinct \\
\hline 67 PIK3СA & ENST0000026396 E12 & $42(1788) \mathrm{C}$ & $A \rightarrow G$ (het) & $62 \%(572)[65 \%(322) / 59 \%(250)]$ & · E (596 rs137902538 (1 & c. $1788 \mathrm{~A}>\mathrm{G}$ & p.Glu596= & distinct \\
\hline 68 TLE4 & ENST0000037655: E18 & $42(2028) \mathrm{C}$ & A $\rightarrow C$ (homo) & $100 \%$ (959) $[100 \%(447) / 100 \%(512)]$ & -A 676 rs34566811 (10 & c. $2028 \mathrm{~A}>\mathrm{C}$ & p.Ala676= & distinct \\
\hline 69 KRAS & ENST0000025607: E2 & $46(35)[\mathrm{cC}$ & $\mathrm{G} \rightarrow \mathrm{A}$ (het) & $48 \%(318) \quad[48 \%(150) / 48 \%(168)]$ & $>\mathrm{D}$ (12) rs121913529 (C & $c .35 \mathrm{G}>\mathrm{A}$ & p.Gly12Asp & distinct \\
\hline 70 LRP1B & ENST0000038948. E32 & $47(5256) \mathrm{C}$ & $A \rightarrow G($ homo $)$ & $100 \%(721)[100 \%(412) / 100 \%(309)]$ & S $(1752$ COSM 4133144 & c. $5256 \mathrm{~A}>\mathrm{G}$ & p.Ser1752= & distinct \\
\hline 71 ARID2 & ENST0000033434. E1 & $52[\mathrm{chr} 12 \mathrm{C}$ & $\mathrm{G} \rightarrow \mathrm{A}$ (het) & $46 \%(6)[50 \%(6) / 0 \%(0)]$ & : UTR & c. $-121 \mathrm{G}>\mathrm{A}$ & & forced, distinct \\
\hline 72 SLC25A24 & ENST0000037004 E1 & 53 [chr1:l C & A $\rightarrow T$ (homo) & $100 \%(91)[100 \%(91) / 0 \%(0)]$ & i' UTR rs524504(1000 & c. $-145 \mathrm{~A}>\mathrm{T}$ & & forced,distinct \\
\hline 73 ROBO2 & ENST0000048769, E2 & $55(42)$ [c C & $A \rightarrow G$ (het) & $79 \%(2134)[76 \%(893) / 82 \%(1241)]$ & $>T$ (14) rs62269817 (db & c. $42 A>G$ & p.Thr14= & distinct \\
\hline 74 SMARCA4 & ENSTO000045071 E5 & 56 (915) |C & $\mathrm{G} \rightarrow \mathrm{A}$ (het) & $95 \%(485)[47 \%(21) / 100 \%(464)]$ & . P(305 rs149573400 (1 & c. $.915 \mathrm{G}>\mathrm{A}$ & p.Pro305 = & distinct \\
\hline 75 LRP1B & ENST0000038948. E27 & $58(4392) \mathrm{C}$ & $A \rightarrow G$ (het) & $43 \%(180)[44 \%(118) / 41 \%(62)]$ & R (146< rs79054985 (10 & c. $4392 \mathrm{~A}>\mathrm{G}$ & p.Arg1464= & distinct \\
\hline 76 КМт2С & ENST0000026218: E15 & $59(2591) \mathrm{C}$ & $A \rightarrow G$ (het) & $35 \%(69) \quad[41 \%(56) / 22 \%(13)]$ & G $(864$ rs4024420 (dbS & c. $2591 \mathrm{~A}>\mathrm{G}$ & p.Glu864Gly & distinct \\
\hline 77 POLR3A & ENST0000037237: E3 & $60(240)$ IC & A $\rightarrow C$ (het) & $74 \%(330)[76 \%(206) / 71 \%(124)]$ & $>\mathrm{L}(80)$ rs $12248310(10$ & c. $240 A>C$ & p.Leu80 $=$ & distinct \\
\hline 78 PBRM1 & ENST00000394831 E23 & $64(3522) \mathrm{C}$ & A $\rightarrow T$ (homo) & $100 \%(904)[100 \%(389) / 100 \%(515)]$ & $\mathrm{P}(117<\cos M 4002747$ & c. $3522 \mathrm{~A}>\mathrm{T}$ & p.Pro1174= & listinct \\
\hline 79 BRD2 & ENST0000044908: E2 & 64 [chr6:l C & $\mathrm{G} \rightarrow \mathrm{A}$ (homo) & $100 \%(405)[100 \%(286) / 100 \%(119)]$ & i' UTR rs516535(1000 & c. $-49 G>A$ & & distinct \\
\hline 80 LRP1B & ENST0000038948. E30 & $67(5006) \mathrm{C}$ & $A \rightarrow C$ (het) & $65 \%(639) \quad[67 \%(399) / 63 \%(240)]$ & $A(1669)$ & c. $5006 \mathrm{~A}>\mathrm{C}$ & p.Glu1669Ala & distinct \\
\hline 81 SLIT2 & ENST0000050415. E21 & $68(2211) \mathrm{C}$ & $C \rightarrow T$ (het) & $28 \%(204)[28 \%(107) / 28 \%(97)]$ & . V (737 rs7690492 (100 & c. $2211 \mathrm{C}>\mathrm{T}$ & p.Val737= & distinct \\
\hline $82 \mathrm{APC}$ & ENST00000257431 E14 & $69(1695) \mathrm{C}$ & $A \rightarrow G$ (homo) & $100 \%(241)[100 \%(102) / 99 \%(139)]$ & - E (565 rs77921116 (10 & c. $1695 \mathrm{~A}>\mathrm{G}$ & p.Glu565= & distinct \\
\hline 83 LRP1B & ENST0000038948, E8 & $69(1082) \mathrm{C}$ & $\mathrm{G} \rightarrow \mathrm{A}$ (het) & $36 \%(279)[35 \%(131) / 36 \%(148)]$ & Q $(361$ COSM1007086 & c. $1082 \mathrm{G}>\mathrm{A}$ & p.Arg361Gln & distinct \\
\hline 84 SLC25A24 & ENST0000056548: E1 & $70 . .76 / 7 \mathrm{k} D$ & CCTGCGC (het) & $65 \%(112)[65 \%(110) / 50 \%(2)]$ & i' UTR rs150513527 (1.- & 151_-145delCCTGC & & forced, distinct \\
\hline 85 FAT1 & ENST0000044180: E23 & $74(12177 ; \mathrm{C}$ & $\mathrm{G} \rightarrow \mathrm{C}$ (homo) & $100 \%(641)[100 \%(300) / 100 \%(341)]$ & $\mathrm{N}(405 \subseteq$ rs1280097 (100 & c. $12177 \mathrm{G}>\mathrm{C}$ & p.Lys4059Asn & distinct \\
\hline 86 RBM6 & ENST0000026602: E21 & 75 (3321) C & $\mathrm{C} \rightarrow \mathrm{T}$ (homo) & $100 \%(371)[100 \%(112) / 100 \%(259)]$ & $Y(110 ;$ COSM 4158172 & c. $3321 \mathrm{C}>\mathrm{T}$ & p. $\operatorname{Tyr} 1107=$ & distinct \\
\hline 87 CHD1 & ENST0000028404: E26 & $77(3648) \mathrm{C}$ & $C \rightarrow T$ (homo) & $100 \%(670)[100 \%(350) / 100 \%(320)]$ & $\mathrm{S}$ (121€ rs140751250 (1 & c. $3648 \mathrm{C}>\mathrm{T}$ & p.Ser1216= & distinct \\
\hline 88 EGFR & ENST0000027549: E20 & $78(2361) \mathrm{C}$ & $\mathrm{G} \rightarrow \mathrm{A}($ homo $)$ & $99 \%(821)[98 \%(427) / 99 \%(394)]$ & . $Q(787$ COSM 1451600 & c. $2361 \mathrm{G}>\mathrm{A}$ & p. $G \ln 787=$ & distinct \\
\hline 89 LRP1B & ENST0000038948. E85 & $80(13047 ; \mathrm{C}$ & $\mathrm{G} \rightarrow \mathrm{A}$ (het) & $22 \%(220)[24 \%(108) / 21 \%(112)]$ & $\mathrm{T}(434 \mathrm{C}$ rs1386356 (100 & c. $13047 \mathrm{G}>\mathrm{A}$ & p.Thr4349= & distinct \\
\hline
\end{tabular}




\begin{tabular}{|c|c|c|c|c|c|c|c|c|}
\hline 0 GNAS & ENST0000037108: E5 & $81(393)$ [C & $C \rightarrow T$ (het) & $81 \%(740)[82 \%(276) / 81 \%(464)]$ & I (131) COSM3758661 & c. $393 C>T$ & p.lle131= & distinct \\
\hline 1 SLC25A24 & ENST0000056548: E4 & $82(480)$ [C & $\mathrm{G} \rightarrow \mathrm{A}$ (het) & $28 \%(139)[25 \%(62) / 31 \%(77)]$ & · E (160' rs11185293 (10 & c. $480 \mathrm{G}>\mathrm{A}$ & p.Glu160= & distinct \\
\hline 92 PIK3CA & ENST0000026396' E6 & $84(1143) \mathrm{C}$ & $C \rightarrow G$ (het) & $63 \%(491) \quad[61 \%(178) / 64 \%(313)]$ & . P (381 rs72561481 (10 & c. $1143 \mathrm{C}>\mathrm{G}$ & p.Pro381= & distinct \\
\hline $93 \mathrm{ROBO} 2$ & ENST0000048769. E2 & $86 . .88$ (73. Indel & GTG -> ATC (het) & $34 \%(891) \quad[32 \%(326) / 36 \%(565)]$ & $>1(25)$ & c.73_75delinsATC & p.Val25lle & distinct \\
\hline 94 КMT2C & ENST0000026218! E18 & $87(2958) \mathrm{C}$ & $A \rightarrow G$ (het) & $51 \%(269)[53 \%(123) / 50 \%(146)]$ & . P (986 rs28439884 (db & c. $2958 \mathrm{~A}>\mathrm{G}$ & p.Pro986= & distinct \\
\hline $95 \mathrm{CHD} 1$ & ENST0000028404! E31 & $87(4335) \mathrm{C}$ & $\mathrm{G} \rightarrow \mathrm{A}$ (homo) & $100 \%(305)[100 \%(112) / 100 \%(193)]$ & $\mathrm{E}(1445$ COSM 4003637 & c. $4335 \mathrm{G}>\mathrm{A}$ & p.Glu1445= & distinct \\
\hline $96 \mathrm{ROBO} 2$ & ENST0000048769. E2 & 88 (75) [c C & $G \rightarrow C$ (het) & $39 \%(977)[36 \%(347) / 42 \%(630)]$ & $>V(25)$ rs62269818 (db & c. $75 \mathrm{G}>\mathrm{C}$ & p.Val25= & distinct \\
\hline 97 LRP1B & ENST0000038948. E51 & $89(8238) \mathrm{C}$ & $\mathrm{G} \rightarrow \mathrm{A}$ (het) & $65 \%(284)[65 \%(127) / 65 \%(157)]$ & G (274trs61732738 (10 & c. $8238 \mathrm{G}>\mathrm{A}$ & p.Gly2746= & distinct \\
\hline 98 SLC25A24 & ENST0000056548: E1 & 89 [chr1:?̨ C & $A \rightarrow C$ (het) & $75 \%(115)[76 \%(113) / 50 \%(2)]$ & : UTR rs554709 (1000 & c. $-132 A>C$ & & forced,distinct \\
\hline 99 КМт2 C & ENST0000026218! E18 & $92(2963) \mathrm{C}$ & $G>T$ (het) & $55 \%(256)[56 \%(112) / 54 \%(144)]$ & . F (988 rs28522267 (db & c. $2963 \mathrm{G}>\mathrm{T}$ & p.Cys988Phe & distinct \\
\hline 100 BRD2 & ENST0000044908: E8 & $92(1280) \mathrm{C}$ & $C \rightarrow T($ homo $)$ & $100 \%(807) \quad[100 \%(418) / 100 \%(389)]$ & - V (427 rs3918143 (100 & c. $1280 C>T$ & p.Ala427Val & distinct \\
\hline D1 SLC25A24 & ENST0000056548: E2 & $93(276)$ [C & $A \rightarrow G$ (het) & $71 \%(195)[70 \%(85) / 73 \%(110)]$ & $>K(92) \operatorname{COSM} 3750202$ & c. $276 A>G$ & p.Lys92= & distinct \\
\hline 102 BRD4 & ENST0000026337' E11 & 95 (2142) C & C $\rightarrow \mathrm{T}$ (homo) & $100 \%(968)[100 \%(444) / 100 \%(524)]$ & . S (714) rs114723577 (1 & c. $2142 C>T$ & p.Ser714= & distinct \\
\hline 103 КМт2C & ENST0000026218! E7 & 97 (946) [C & $A \rightarrow T$ (het) & $83 \%(821) \quad[83 \%(469) / 82 \%(352)]$ & · S (316' rs10454320 (db & c.946A $>\mathrm{T}$ & p.Thr316Ser & distinct \\
\hline 104 ATM & ENST0000027861। E23 & $99(3383) \mathrm{C}$ & $A \rightarrow G($ homo $)$ & $100 \%(523)[99 \%(136) / 100 \%(387)]$ & $\mathrm{R}(112\{\cos M 1350830$ & c. $3383 \mathrm{~A}>\mathrm{G}$ & p.GIn1128Arg & distinct \\
\hline 105 FAT1 & ENST0000044180: E26 & $101(1310: C$ & $\mathrm{T}>\mathrm{C}$ (homo) & $100 \%(840)[100 \%(404) / 100 \%(436)]$ & S $(4367$ COSM 4158952 & c. $13101 \mathrm{~T}>\mathrm{C}$ & p.Ser4367= & distinct \\
\hline 106 SMARCA4 & ENST0000045071' E8 & $105(1524) \mathrm{C}$ & $\mathrm{T} \rightarrow \mathrm{C}$ (homo) & $100 \%(955) \quad[100 \%(480) / 100 \%(475)]$ & · H (508 COSM4131184 & c.1524T>C & p.t $[c .1524 T>C]$ & distinct \\
\hline 107 SMAD4 & ENST0000034298: E9 & $112(1067) \mathrm{C}$ & C $\rightarrow \mathrm{T}$ (homo) & $100 \%(733) \quad[100 \%(368) / 100 \%(365)]$ & $\cdot \mathrm{L}(356 ;$ COSM14049 (Cl & c.1067C>T & p.Pro356Leu & distinct \\
\hline 108 FAT1 & ENST0000044180: E19 & 112 (1066ic & $\mathrm{T} \rightarrow \mathrm{G}$ (homo) & $100 \%$ (779) $[100 \%(403) / 100 \%(376)]$ & A (3554 rs2637777 (100 & c.10660T>G & p.Ser3554Ala & distinct \\
\hline 109 LRP1B & ENST0000038948. E16 & $113(2616) \mathrm{C}$ & $C \rightarrow T($ homo) & $100 \%$ (160) $[100 \%(76) / 100 \%(84)]$ & - D (872 rs13007735 (10 & c. $2616 C>T$ & p.Asp872= & distinct \\
\hline 110 FGFR2 & ENST0000035848' E1 & 117 [chr1C & $A \rightarrow G$ (het) & $44 \%(65)[44 \%(23) / 44 \%(42)]$ & : UTR rs41258305(10 & c. $-157 \mathrm{~A}>\mathrm{G}$ & & distinct \\
\hline 111 EGFR & ENST0000027549: E15 & $117(1839) \mathrm{C}$ & C $\rightarrow T$ (homo) & $100 \%(676)[100 \%(311) / 100 \%(365)]$ & A 1613 rs17290169 (10 & c. $1839 C>T$ & p.Ala613= & distinct \\
\hline 112 TLE4 & ENST0000037655: E15 & $118(1458) \mathrm{C}$ & $\mathrm{C} \rightarrow \mathrm{T}$ (homo) & $98 \%(1502)[98 \%(751) / 98 \%(751)]$ & $\cdot H(486$ rs61742686 (10 & c. $1458 \mathrm{C}>\mathrm{T}$ & p.His486= & distinct \\
\hline 113 SF3B1 & ENST0000033550: E24 & $118(3657) \mathrm{C}$ & $A \rightarrow G($ homo $)$ & $100 \%(721)[100 \%(271) / 100 \%(450)]$ & $V(121 \subseteq$ COSM 3757859 & c. $3657 \mathrm{~A}>\mathrm{G}$ & p.Val1219= & distinct \\
\hline 114 SLIT2 & ENST0000050415, E1 & 120 [chr4 C & $G-T$ (het) & $26 \%(10)[26 \%(10) / 0 \%(0)]$ & : UTR rs7655084(100 & c. $-133 \mathrm{G}>\mathrm{T}$ & & forced,di \\
\hline 115 ТР53ВР2 & ENST0000034353' E6 & $121(595) \mathrm{C}$ & $\mathrm{G} \rightarrow \mathrm{A}$ (het) & $60 \%(380)[58 \%(165) / 61 \%(215)]$ & M (199 rs146703239 (1 & c. $595 \mathrm{G}>\mathrm{A}$ & p.Val199Met & distinct \\
\hline 116 FAT1 & ENST0000044180: E13 & $122(9351) \mathrm{C}$ & $\mathrm{T}>\mathrm{C}$ (homo) & $100 \%(1066)[100 \%(477) / 100 \%(589)]$ & D (311: COSM4003024 & c. $9351 \mathrm{~T}>\mathrm{C}$ & p.Asp3117= & distinct \\
\hline 117 SMARCC2 & ENST0000055016. E25 & $134(2682) \mathrm{C}$ & $\mathrm{C} \rightarrow \mathrm{T}$ (homo) & $100 \%(291)[100 \%(101) / 100 \%(190)]$ & · A (894 rs17852368 (10 & c. $2682 \mathrm{C}>\mathrm{T}$ & p.Ala894= & distinct \\
\hline 118 FAT1 & ENST0000044180: E13 & $134(9363) \mathrm{C}$ & $\mathrm{C} \rightarrow \mathrm{T}$ (homo) & $100 \%(1039)[100 \%(413) / 100 \%(626)]$ & N (312: rs2249916 (100 & c.9363C>T & p.Asn3121= & distinct \\
\hline 119 SF3B1 & ENST0000033550: E18 & $135(2631) \mathrm{C}$ & $\mathrm{T} \rightarrow \mathrm{C}$ (homo) & $100 \%(412)[100 \%(128) / 100 \%(284)]$ & - $\mathrm{G}(877$ COSM3757860 & c. $2631 \mathrm{~T}>\mathrm{C}$ & p.Gly $877=$ & distinct \\
\hline 120 BRD7 & ENST0000039468: E7 & $144(846) \mathrm{C}$ & $C \rightarrow T$ (het) & $50 \%(356) \quad[51 \%(163) / 50 \%(193)]$ & - A (282 COSM3720984 & c. $846 \mathrm{C}>\mathrm{T}$ & p.Ala282 = & distinct \\
\hline 121 U2AF2 & ENST0000045055, E11 & $159(1191) \mathrm{C}$ & $\mathrm{C} \rightarrow \mathrm{T}$ (homo) & $99 \%(507)[99 \%(193) / 99 \%(314)]$ & - D (397 COSM1396639 & c.1191C>T & p.Asp397= & distinct \\
\hline 122 TP53 & ENST0000026930: E4 & $171(267), D$ & $\mathrm{C}$ (homo) & $95 \%(635)[91 \%(272) / 98 \%(363)]$ & 1122 R COSM1180853 & c.267delc & p.Ser90Profs $* 33$ & distinct \\
\hline 123 FAT1 & ENST0000044180: E5 & $176(3818) \mathrm{C}$ & $A \rightarrow G($ homo $)$ & $100 \%(991) \quad[100 \%(478) / 100 \%(513)]$ & R (127: rs328418 (1000 & c. $3818 \mathrm{~A}>\mathrm{G}$ & p.His1273Arg & distinct \\
\hline 124 FAT4 & ENST0000039432: E4 & $191(5760) \mathrm{C}$ & $\mathrm{T}->\mathrm{C}$ (homo) & $100 \%(627)[100 \%(295) / 100 \%(332)]$ & D $(192(\cos M 1131273$ & c.5760T>C & p.Asp1920= & distinct \\
\hline 125 FGFR1 & ENST0000044771: E1 & 192 [chr8 C & C $\rightarrow \mathrm{T}$ (homo) & $100 \%(13)[100 \%(10) / 100 \%(3)]$ & i UTR rs2445003(100 & c. $-751 \mathrm{C}>\mathrm{T}$ & & distinct \\
\hline 126 FGFR2 & ENST0000036905। E17 & 196 [chr1C & $A \rightarrow G($ homo $)$ & $85 \%(23)[0 \%(0) / 85 \%(23)]$ & : UTR rs1649167(100 & c. ${ }^{*} 190 \mathrm{~A}>\mathrm{G}$ & & forced, distinct \\
\hline 127 LRP1B & ENST0000038948. E41 & $206(6633) \mathrm{C}$ & $A \rightarrow T$ (het) & $32 \%(220)[37 \%(102) / 29 \%(118)]$ & P (2211 rs13431727 (10 & c. $6633 \mathrm{~A}>\mathrm{T}$ & p.Pro2211= & distinct \\
\hline 128 FAT1 & ENST0000044180: E19 & $209\left(10755^{\circ} \mathrm{C}\right.$ & $T>C$ (het) & $63 \%(545) \quad[63 \%(259) / 63 \%(286)]$ & T (358t rs115705222 (1 & c. $10757 \mathrm{~T}>\mathrm{C}$ & p.Met3586Thr & distinct \\
\hline 129 BRD3 & ENST0000030340 E6 & $222(936) \mathrm{C}$ & A $\rightarrow G$ (homo) & $100 \%(933)[100 \%(440) / 100 \%(493)]$ & $\cdot L(312)$ COSM4163507 & c. $936 \mathrm{~A}>\mathrm{G}$ & p.Leu312= & distinct \\
\hline 130 TGFBR2 & ENST0000029575. E1 & 255 [chr3 C & $C \rightarrow G$ (het) & $76 \%(212)[76 \%(192) / 83 \%(20)]$ & : UTR rs2306856(100 & c. $-128 \mathrm{C}>\mathrm{G}$ & & tinct \\
\hline 131 FAT4 & ENST0000039432: E9 & $257(7701) \mathrm{C}$ & $\mathrm{G} \rightarrow \mathrm{C}($ homo $)$ & $100 \%(817) \quad[100 \%(399) / 100 \%(418)]$ & V (256; rs988863 (1000 & c. $7701 \mathrm{G}>\mathrm{C}$ & p.Val2567= & distinct \\
\hline 132 BANF1 & ENST0000031217: E1 & 260 [chr1 C & $G \rightarrow C$ (het) & $50 \%(449) \quad[51 \%(255) / 49 \%(194)]$ & : UTR rs1786171 (100 & c. $-249 \mathrm{G}>\mathrm{C}$ & & distinct \\
\hline 133 BANF1 & ENST0000031217: E3 & 275 [chr1c & $C \rightarrow A$ (het) & $52 \%(123)[54 \%(19) / 52 \%(104)]$ & : UTR rs144367403(1 & c. ${ }^{*} 128 \mathrm{C}>\mathrm{A}$ & & distinct \\
\hline 134 PBRM1 & ENST0000039483। E17 & $287(2211) C$ & A $\rightarrow G($ homo $)$ & $100 \%(879) \quad[100 \%(457) / 100 \%(422)]$ & · T (737' COSM1566683 & c. $2211 \mathrm{~A}>\mathrm{G}$ & p.Thr737= & distinct \\
\hline 135 SLIT2 & ENST0000050415، E37 & $293[\mathrm{chr} 4 \mathrm{C}$ & $\mathrm{G} \rightarrow \mathrm{A}$ (homo) & $100 \%(809) \quad[100 \%(303) / 100 \%(506)]$ & UTR rs1379659(100 & c. $* 51 G>A$ & & distinct \\
\hline 136 FGFR1 & ENST0000044771: E1 & 307 [chr8 C & $C \rightarrow T$ (het) & $33 \%(10)[35 \%(8) / 29 \%(2)]$ & : UTR rs3213849(100 & c. $-636 \mathrm{C}>\mathrm{T}$ & & forced,distinct \\
\hline 137 LMNA & ENST00000368301 E12 & 392 [chr1 C & $C \rightarrow T$ (het) & $75 \%(6)[67 \%(2) / 80 \%(4)]$ & UTR rs74116489(10 & c. ${ }^{*} 365 \mathrm{C}>\mathrm{T}$ & & distinct \\
\hline 138 SETD2 & ENST0000040979: E3 & $470(557) \mathrm{C}$ & $\mathrm{C} \rightarrow \mathrm{T}$ (homo) & $100 \%(590) \quad[100 \%(268) / 100 \%(322)]$ & $\cdot$ L (186' rs78759480 (10 & c. $557 \mathrm{C}>\mathrm{T}$ & p.Pro186Leu & distinct \\
\hline 139 ACVR2A & ENST0000024141। E1 & $472 / 1$ bp I (Dup) & $T$ (het) & $33 \%(4)[33 \%(4) / 0 \%(0)]$ & UTR & c.-166dupT & & forced, distinct \\
\hline 140 BRD2 & ENST0000037482! E2 & 523 [chr6C & $\mathrm{G} \rightarrow \mathrm{T}$ (homo) & $100 \%(312)[100 \%(57) / 100 \%(255)]$ & :UTR rs974357(1000 & c. $-782 \mathrm{G}>\mathrm{T}$ & & distinct \\
\hline 141 MARK2 & ENST0000040201। E19 & 548 [chr1C & $A \rightarrow C$ (het) & $47 \%(28)[0 \%(0) / 47 \%(28)]$ & UTR rs182664(1000 & c. ${ }^{*} 187 \mathrm{~A}>\mathrm{C}$ & & forced, distinct \\
\hline 142 RNF43 & ENST0000040797' E9 & $633(1585) \mathrm{C}$ & $C \rightarrow T$ (het) & $75 \%$ (1042) $[76 \%(639) / 74 \%(403)]$ & W (52s CosM191575 (c & c. $1585 C>T$ & p.Arg529Trp & distinct \\
\hline 143 MYC & ENST0000037797I E2 & 708 (738) C & $\mathrm{G} \rightarrow \mathrm{A}$ (het) & $55 \%(945) \quad[50 \%(303) / 58 \%(642)]$ & · P (246 rs2070582 (100 & c. $738 \mathrm{G}>\mathrm{A}$ & p.Pro246= & distinct \\
\hline 144 MYC & ENST0000037797। E2 & $708(738) \mathrm{C}$ & $G->A$ (het) & $55 \%(945)[50 \%(303) / 58 \%(642)]$ & . P (246 rs2070582 (100 & c. $738 \mathrm{G}>\mathrm{A}$ & p.Pro246= & distinct \\
\hline 145 FAT1 & ENST0000044180: E27 & $883[\mathrm{chr} 4 \mathrm{C}$ & $\mathrm{G} \rightarrow \mathrm{A}$ (het) & $30 \%(9)[0 \%(0) / 30 \%(9)]$ & :UTR rs7680937(100 & c. ${ }^{2} 254 \mathrm{G}>\mathrm{A}$ & & forced, distinct \\
\hline 146 LRP1B & ENST0000038948، E1 & $895[\mathrm{chr} 2 \mathrm{C}$ & C $>\mathrm{G}$ (homo) & $99 \%(705) \quad[99 \%(453) / 99 \%(252)]$ & : UTR rs1375610(100 & c. $-78 \mathrm{C}>\mathrm{G}$ & & distinct \\
\hline 147 KMT2D & ENST0000030106 E10 & 1025..105: D & ATCTCCGCAGGCT & T 74\% (366) [55\% (98) / 85\% (268)] & L -> L (i rs375538882 (dlA & TCTCCGCAGGC & c.p.Ala765 Gl & distinct \\
\hline 148 KMT2D & ENST0000030106 E10 & 1025..105: D & ATCTCCGCAGGCT & $74 \%(366) \quad[55 \%(98) / 85 \%(268)]$ & $L \rightarrow L$ (i rs 375538882 (dlA & TCCGCAGGCT & p.Ala765_c & distinct \\
\hline 149 TLE4 & ENST0000037655: E1 & $1063(45) \mathrm{C}$ & $A \rightarrow G($ homo $)$ & $100 \%(751)[100 \%(291) / 100 \%(460)]$ & $>P(15)$ COSM4164047 & c. $45 \mathrm{~A}>\mathrm{G}$ & p.Pro15= & distinct \\
\hline 150 FAT1 & ENST0000044180: E2 & $1230(121: \mathrm{C}$ & $T \rightarrow G$ (het) & $28 \%(257)[30 \%(160) / 26 \%(97)]$ & $R(404$ COSM4003044 & c. $1212 \mathrm{~T}>\mathrm{G}$ & p.Ser404Arg & distinct \\
\hline 151 FAT4 & ENST0000039432! E1 & $1371(135: \mathrm{C}$ & A $\rightarrow T$ (homo) & $100 \%(1316)[100 \%(665) / 100 \%(651)]$ & $>$ L (453 COSM3760369 & c.1358A>T & p.Gln453Leu & distinct \\
\hline 152 FAT4 & ENST0000039432! E9 & $1392(8831 \mathrm{C}$ & $A \rightarrow G$ (het) & $59 \%(484) \quad[59 \%(245) / 59 \%(239)]$ & $V(2946$ rs76048257 (10 & c. $8836 \mathrm{~A}>\mathrm{G}$ & p.lle2946Val & distinct \\
\hline 153 FAT1 & ENST0000044180: E2 & 1462 (144. C & $\mathrm{G} \rightarrow \mathrm{A}$ (het) & $29 \%(308)[27 \%(134) / 31 \%(174)]$ & $>$ I (482) rs3733413 (100 & c. $1444 \mathrm{G}>\mathrm{A}$ & p.Val482Ile & distinct \\
\hline 154 FAT1 & ENST0000044180: E2 & $1503(148 ! \mathrm{C}$ & $C \rightarrow T$ (het) & $28 \%(334) \quad[27 \%(174) / 30 \%(160)]$ & $\cdot \mathrm{N}(495$ COSM 4003040 & c. $1485 C>T$ & p.Asn495= & distinct \\
\hline 155 FAT4 & ENST0000039432! E17 & $1520(145 ! \mathrm{C}$ & $A \rightarrow G$ (het) & $64 \%(560) \quad[67 \%(271) / 61 \%(289)]$ & R (486f rs1014866 (100 & c. $14598 \mathrm{~A}>\mathrm{G}$ & p.Arg4866= & distinct \\
\hline 156 APC & ENST00000257431 E16 & $1774(373:$ C & A $\rightarrow G($ homo $)$ & $100 \%(578) \quad[100 \%(242) / 100 \%(336)]$ & Q (124، rs74380081 (10 & c.3732A $>\mathrm{G}$ & p.G $\ln 1244=$ & distinct \\
\hline 157 FAT4 & ENST0000039432! E17 & $1836(149 \mathrm{C}$ & $C \rightarrow T$ (het) & $36 \%(193) \quad[40 \%(65) / 34 \%(128)]$ & S (4972 COSM4002932 & c. $14914 C>T$ & p.Pro4972Ser & distinct \\
\hline 158 FAT1 & ENST0000044180: E2 & $1860(184: \mathrm{C}$ & $C \rightarrow G$ (het) & $30 \%(216)[30 \%(108) / 30 \%(108)]$ & $\cdot$ L $(614 ;$ COSM4003038 & c. $1842 C>G$ & p.Phe614Leu & distinct \\
\hline 159 FAT4 & ENST0000039432! E9 & $2426(9871 \mathrm{C}$ & $\mathrm{G}>\mathrm{A}$ (het) & $63 \%(385)[61 \%(169) / 65 \%(216)]$ & G (329) rs115219562 (1 & c. $9870 \mathrm{G}>\mathrm{A}$ & p.Gly3290= & distinct \\
\hline 160 FAT4 & ENST0000039432! E1 & $2433(2421 \mathrm{C}$ & $C \rightarrow T$ (homo) & $100 \%(968)[100 \%(592) / 100 \%(376)]$ & $\cdot \mathrm{V}(807$ COSM3760371 & $c .2420 C>T$ & p.Ala807Val & distinct \\
\hline 161 FAT1 & ENST0000044180: E2 & $2602(258 \cdot \mathrm{C}$ & G $>C$ (het) & $33 \%(376) \quad[33 \%(212) / 33 \%(164)]$ & $\cdot$ L (862 COSM4003037 & c. $2584 \mathrm{G}>\mathrm{C}$ & p.Val862Leu & distinct \\
\hline 162 SETD2 & ENST0000040979: E3 & 2617 (270. C & $\mathrm{G} \rightarrow \mathrm{C}$ (homo) & $100 \%(961) \quad[100 \%(526) / 100 \%(435)]$ & $\mathrm{Q}(902 \mathrm{rs} 58906143(10$ & c. $2704 \mathrm{G}>\mathrm{C}$ & p.Glu902GIn & distinct \\
\hline 163 FAT4 & ENST0000039432! E1 & $2824(281: C$ & $\mathrm{G} \rightarrow \mathrm{T}$ (het) & $26 \%(284) \quad[26 \%(136) / 26 \%(148)]$ & N (937 rs112454576 (1 & $c .2811 \mathrm{G}>\mathrm{T}$ & p.Lys937Asn & inct \\
\hline 164 FAT4 & ENST0000039432! E1 & $2957(294 . C$ & $\mathrm{T}>\mathrm{C}$ (homo) & $100 \%(667) \quad[100 \%(345) / 100 \%(322)]$ & $\cdot$ L (982) rs2940779 (100 & c. $2944 \mathrm{~T}>\mathrm{C}$ & p.Leu982= & distinct \\
\hline 165 FAT1 & ENST0000044180: E2 & $3208(3191 \mathrm{C}$ & $A \rightarrow G($ homo $)$ & $100 \%(959) \quad[100 \%(479) / 100 \%(480)]$ & G $(106<$ COSM4003035 & c.3190A>G & p.Arg1064Gly & distinct \\
\hline 166 FAT1 & ENST0000044180: E10 & $3988(879: \mathrm{C}$ & $A \rightarrow T$ (het) & $71 \%(575) \quad[70 \%(256) / 73 \%(319)]$ & L (293ミrs1280098 (100 & c.8798A $>\mathrm{T}$ & p.Gln2933Leu & inct \\
\hline 167 FAT1 & ENST0000044180: E10 & $3988(879: \mathrm{C}$ & $A \rightarrow C$ (het) & $29 \%(231) \quad[30 \%(111) / 27 \%(120)]$ & $\mathrm{P}(293:$ COSM4005727 & c. $8798 \mathrm{~A}>\mathrm{C}$ & p.Gln2933Pro & distinct \\
\hline 168 FAT4 & ENST0000039432! E9 & $4038(114: C$ & $A \rightarrow G$ (het) & $64 \%(611) \quad[63 \%(290) / 65 \%(321)]$ & E (382\& rs17009684 (10 & c. $11482 A>G$ & p.Lys3828Glu & distinct \\
\hline 169 FAT4 & \$T0000039432! E9 & $4174(116 \mathrm{C}$ & $\mathrm{G} \rightarrow \mathrm{A}$ (homo) & $100 \%$ (916) $[100 \%(454) / 100 \%(462$ & N (387ः rs12650153 (10 & c. $11618 \mathrm{G}>\mathrm{A}$ & p.Ser3873Asn & \\
\hline 170 FAT4 & ENST0000039432! E1 & $4318(430 ! \mathrm{C}$ & $\mathrm{C} \rightarrow \mathrm{T}$ (homo) & $100 \%(1097) \quad[100 \%(536) / 100 \%(561)]$ & I(1435 rs2710555 (100 & c. $4305 C>T$ & p.lle1435= & distinct \\
\hline 171 LRP1B & ENST0000038948، E55 & $+3[\mathrm{chr} 2:$ : C & $A \rightarrow G$ (het) & $24 \%(72)[27 \%(20) / 23 \%(52)]$ & rs117225004 (1 & c. $8850+3 A>G$ & & distinct \\
\hline 172 ROBO1 & ENST0000046423: E26 & $+4[\mathrm{chr} 3: \mathrm{C}$ & $\mathrm{T} \rightarrow \mathrm{C}$ (homo) & $100 \%(679) \quad[100 \%(303) / 100 \%(376)]$ & rs7636043 (100 & c. $3875+4 \mathrm{~T}>\mathrm{C}$ & & tinct \\
\hline 173 LRP1B & ENST0000038948، E33 & $+5[\mathrm{chr} 2: \mathrm{l} \mathrm{C}$ & $A \rightarrow G($ homo $)$ & $100 \%(380)[100 \%(144) / 100 \%(236)]$ & rs996361(1000 & c. $5500+5 \mathrm{~A}>\mathrm{G}$ & & distinct \\
\hline 174 PBRM1 & ENST00000394831 E29 & $+6 / 3 b p$ & GAG (homo) & $88 \%(469)[91 \%(166) / 87 \%(303)]$ & rs71084187 (db4 & $76+5 \_4576+6$ ins $G$ & & distinct \\
\hline 175 SF1 & ENST00000377391 E6 & $+6[\mathrm{chr} 11 \mathrm{C}$ & $C \rightarrow T$ (het) & $48 \%(736) \quad[52 \%(424) / 42 \%(312)]$ & rs148875917 (1 & c. $663+6 C>T$ & & distinct \\
\hline 176 GATA6 & ENST0000026921। E6 & $+7[\mathrm{chr} 18 \mathrm{C}$ & $A \rightarrow G($ homo $)$ & $100 \%(219)[100 \%(53) / 100 \%(166)]$ & COSM4000377 & c. $1620+7 A>G$ & & tinct \\
\hline 177 LRP1B & ENST0000038948، E19 & $+10[\mathrm{chr} 2 \mathrm{C}$ & $A \rightarrow C$ (homo) & $100 \%(217)[100 \%(78) / 100 \%(139)]$ & rs1429348 (100 & c. $2968+10 A>C$ & & tinct \\
\hline 178 LRP1B & ENST0000038948، E49 & $+12[\mathrm{chr} 2 \mathrm{C}$ & A $\rightarrow T$ (het) & $63 \%(347) \quad[62 \%(101) / 64 \%(246)]$ & rs13404083 (10 & $c .8026+12 A>T$ & & distinct \\
\hline 179 LMNA & ENST0000036830I E4 & +13 [chr1 C & $\mathrm{G} \rightarrow \mathrm{T}$ (het) & $61 \%(576) \quad[57 \%(227) / 64 \%(349)]$ & rs11264444 (10 & c. $810+13 \mathrm{G}>\mathrm{T}$ & & istinct \\
\hline
\end{tabular}




\begin{tabular}{|c|c|c|c|c|c|c|c|c|c|}
\hline 180 LRP1B & ENST0000038948: E30 & $+14 / 1 \mathrm{bp} \mathrm{I}$ & $A$ (het) & $87 \%(451) \quad[79 \%(152) / 92 \%(299)]$ & \multicolumn{4}{|c|}{ rs78477398 $(10.5114+13$ _5114+14insA } & distinct \\
\hline 181 LRP1B & ENST0000038948: E34 & $+15[\mathrm{chr} 2 \mathrm{C}$ & $A-G$ (het) & $71 \%(411) \quad[72 \%(155) / 70 \%(256)]$ & & rs13382825 (10 & c. $5626+15 A>G$ & & distinct \\
\hline 182 МҮСВР2 & ENST0000035733' E42 & $+15 / 1 \mathrm{bp} \mathrm{D}$ & $\mathrm{T}$ (homo) & $95 \%(242)[100 \%(33) / 95 \%(209)]$ & & rs397840781 (d & c.6226+15delT & & distinct \\
\hline 183 КМT2C & ENST0000026218! E7 & $+15[\mathrm{chr} 7 \mathrm{C}$ & $\mathrm{G} \rightarrow \mathrm{C}$ (het) & $24 \%(198)[23 \%(59) / 24 \%(139)]]$ & & rs201635031 (d & c. $1012+15 G>C$ & & distinct \\
\hline $184 \mathrm{CHD} 1$ & ENST0000028404: E13 & +16 [chr5 C & A $\rightarrow G($ homo $)$ & $100 \%(337)[100 \%(93) / 100 \%(244)]$ & & rs17166428(10 & c. $1991+16 A>G$ & & distinct \\
\hline 185 SLIT2 & ENST0000050415, E28 & $+16[\mathrm{chr} 4 \mathrm{C}$ & $A \rightarrow G$ (het) & $23 \%(102)[25 \%(41) / 22 \%(61)]$ & & rs12506323 (10 & $c .2948+16 A>G$ & & distinct \\
\hline 186 MARK2 & ENST0000040201। E11 & +16 [chr1C & $C \rightarrow T$ (het) & $51 \%(405)[50 \%(114) / 51 \%(291)]$ & & rs224174 (1000 & $c .1101+16 C>T$ & & distinct \\
\hline 187 SMARCA4 & ENST0000045071' E31 & $+16[\mathrm{chr} 1 \mathrm{C}$ & G -> T (homo) & $100 \%(565)[100 \%(282) / 100 \%(283)]$ & & rs151265814 (1 & c. $4675+16 G>T$ & & distinct \\
\hline 188 NARF & ENST0000030979. E3 & $+17[\mathrm{chr} 1 \mathrm{C}$ & $A \rightarrow T$ (het) & $74 \%(577) \quad[73 \%(149) / 74 \%(428)]$ & & rs28401416 (10 & c. $252+17 A>T$ & & distinct \\
\hline 189 ROBO3 & ENST0000039780 E4 & $+24[\mathrm{chr} 1 \mathrm{C}$ & $\mathrm{T} \rightarrow \mathrm{C}$ (homo) & $100 \%(793)[100 \%(417) / 100 \%(376)]$ & & rs4936957(100 & c. $766+24 \mathrm{~T}>\mathrm{C}$ & & distinct \\
\hline 190 SF1 & ENST00000377391 E6 & $+32[\mathrm{chr} 1 \mathrm{C}$ & A $>$ G (homo) & $100 \%(1454) \quad[100 \%(607) / 100 \%(847)]$ & & rs484886 (1000 & c. $663+32 A>G$ & & distinct \\
\hline 191 POLR3A & ENST0000037237 E22 & $+42[\mathrm{chr} 1 \mathrm{C}$ & $\mathrm{T} \rightarrow \mathrm{C}$ (het) & $59 \%(449) \quad[58 \%(226) / 60 \%(223)]$ & & rs3815891 (100 & $c .2988+42 T>C$ & & distinct \\
\hline $192 \mathrm{BRD} 2$ & ENST0000044908: E9 & $+663 / 4 \mathrm{br} \mathrm{I}$ (Dup) & GTTT (het) & $56 \%(76)[79 \%(55) / 32 \%(21)]$ & & c. 1700 & $+659 \_1700+662$ dup & OGTTT & distinct \\
\hline 193 BRD2 & ENST0000044908: E9 & $+672[\mathrm{chr} C$ & $A \rightarrow T$ (het) & $84 \%(97)[85 \%(52) / 82 \%(45)]$ & & rs2395380 (100 & c. $1700+672 A>T$ & & distinct \\
\hline 194 BRD2 & ENST0000044908: E9 & $+689[\mathrm{chr} C$ & G -> T (homo) & $95 \%(109)[91 \%(52) / 98 \%(57)]$ & & rs2082260 (100 & c. $1700+689 \mathrm{G}>\mathrm{T}$ & & distinct \\
\hline 195 BRD2 & ENST0000044908: E9 & +698 / 1 br I (Dup) & $T$ (het) & $40 \%(47)[40 \%(20) / 40 \%(27)]$ & & & c.1700+697dupT & & distinct \\
\hline 196 SLIT2 & ENST0000050415. E1 & $+1105[\mathrm{ct} C$ & $\mathrm{G} \rightarrow \mathrm{A}$ (het) & $28 \%(334) \quad[29 \%(137) / 27 \%(197)]$ & & rs1323066 (100 & c. $179+1105 \mathrm{G}>\mathrm{A}$ & & istinct \\
\hline 197 SMAD4 & ENST0000034298: E4 & $+2088[\mathrm{ct} C$ & $\mathrm{G}->\mathrm{C}$ (homo) & $100 \%(89) \quad[100 \%(42) / 100 \%(47)]$ & & rs7229678(100 & c. $454+2088 \mathrm{G}>\mathrm{C}$ & $2 / 1$ of 3 & distinct \\
\hline 198 SMAD3 & ENST0000032736' E1 & $+32698 / 1 \mathrm{D}$ & $\mathrm{T}$ (homo) & $98 \%(1802)[98 \%(797) / 97 \%(1005)]$ & & & c.206+32698delT & & distinct \\
\hline 199 ATM & ENST0000027861। E1 & 275 [chr1C & $\mathrm{G}->\mathrm{A}($ homo) & $100 \%(280) \quad[100 \%(140) / 100 \%(140)]$ & : UTR & rs189037 (1000 & c. $-111 \mathrm{G}>\mathrm{A}$ & $1 / 0 \mathrm{DP}$ & distinct \\
\hline 200 BRCA1 & ENST0000035765، E10 & $1942(261: C$ & $\mathrm{C} \rightarrow \mathrm{T}($ homo) & $100 \%$ (1429) $[100 \%(679) / 100 \%(750)]$ & $\cdot L(871$ & COSM148278 & c. $2612 C>T$ & p.F 32/ DP, POL & Y distinct \\
\hline 201 ASCC3 & ENST0000036916: E12 & $33(1935) \mathrm{C}$ & C $\rightarrow \mathrm{T}$ (homo) & $100 \%(525)[100 \%(343) / 99 \%(182)]$ & $\cdot L(645$ & rs41288423 (10 & c. $1935 \mathrm{C}>\mathrm{T}$ & p.L 88/Ident SI & I distinct \\
\hline 202 MMACHC & ENST0000040106 E3 & $45(321)$ IC & $\mathrm{G} \rightarrow \mathrm{A}$ (het) & $29 \%(242)[28 \%(107) / 31 \%(135)]$ & $\cdot v(107$ & rs2275276 (100 & c. $321 \mathrm{G}>\mathrm{A}$ & p.l 77/Ident-S & $\mathrm{N}$ distinct \\
\hline $203 \mathrm{TPH} 2$ & ENST0000033385। E9 & $57(1125) \mathrm{C}$ & A $\rightarrow T$ (homo) & $100 \%(895) \quad[100 \%(523) / 100 \%(372)]$ & - A (375 & COSM3753489 & c. $1125 \mathrm{~A}>\mathrm{T}$ & p.A 16 Z Ident-S & $N$ distinct \\
\hline 204 EDNRB & ENST0000044657: E4 & $30(831)$ IC & A $>$ G (homo) & $100 \%(499) \quad[100 \%(286) / 100 \%(213)]$ & $\cdot \mathrm{L}(277$ & COSM3753750 & c. $831 \mathrm{~A}>\mathrm{G}$ & p.L $15 €$ Ident-S & $N$ distinct \\
\hline 205 GABRG3 & ENST0000033374: E8 & $98(963)$ IC & $C \rightarrow T$ (het) & $37 \%(629) \quad[37 \%(366) / 36 \%(263)]$ & $\cdot \mathrm{T}(321$ & rs140679 (1000 & $c .963 C>T$ & p.T 10C Ident-S & $\mathrm{N}$ distinct \\
\hline 206 GABRG3 & ENST0000033374: E8 & $98(963)$ /C & C $\rightarrow T$ (het) & $37 \%(629) \quad[37 \%(366) / 36 \%(263)]$ & . T(321 & rs140679 (1000 & c. $963 C>T$ & p.T $10 C$ Ident-S & $\mathrm{N}$ distinct \\
\hline 207 АTP13A4 & ENST0000039244: E6 & $10(543)$ IC & $A \rightarrow G$ (het) & $29 \%(249)[30 \%(163) / 28 \%(86)]$ & M (181 & COSM3759936 & c. $543 \mathrm{~A}>\mathrm{G}$ & p.ll 55/Ident-S & $N$ distinct \\
\hline 208 WDFY3 & ENST0000032236। E6 & $32(336)$ IC & $A \rightarrow G$ (het) & $66 \%(523)[67 \%(280) / 65 \%(243)]$ & $\cdot L(112$ & COSM4003176 & $c .336 \mathrm{~A}>\mathrm{G}$ & p.L 56/Ident-S & $N$ distinct \\
\hline 209 MUT & ENST0000027481: E3 & $251(636) \mathrm{C}$ & $\mathrm{G}->\mathrm{A}($ homo) & $100 \%(435)[100 \%(226) / 99 \%(209)]$ & $\cdot \mathrm{K}(212$ & rs2229384 & c. $636 \mathrm{G}>\mathrm{A}$ & p.L 151 Ident-S & $N$ distinct \\
\hline $210 \mathrm{MPDZ}$ & ENST0000031921 E25 & $157(3609) \mathrm{C}$ & $A \rightarrow G$ (het) & $29 \%(168)[31 \%(69) / 27 \%(99)]$ & $\mathrm{K}(120$ & Ess10756457 (10 & c. $3609 \mathrm{~A}>\mathrm{G}$ & p.L 47/ Ident-S & $N$ distinct \\
\hline 211 LMNA & ENST00000368301 E9 & $-41[\mathrm{chr} 1 \mathrm{C}$ & $\mathrm{C} \rightarrow \mathrm{T}$ (homo) & $100 \%(1131) \quad[100 \%(705) / 99 \%(426)]$ & & rs553016 (1000 & c. $1489-41 C>T$ & 2/5 POLY & distinct \\
\hline 212 SMARCA2 & ENST0000034972 E33 & -20 [chr9 C & $\mathrm{T} \rightarrow \mathrm{C}$ (homo) & $100 \%$ (123) $[100 \%(82) / 100 \%(41)]$ & & rs3818384 (100 & c. $4595-20 T>C$ & 0/2 POLY & distinct \\
\hline 213 CHD6 & ENST0000037323: E14 & $-19[\mathrm{chr} 21 \mathrm{C}$ & $\mathrm{G}->\mathrm{A}($ homo) & $100 \%(974) \quad[100 \%(600) / 100 \%(374)]$ & & rs4812516(100 & c.1858-19G>A & 0/1 POLY & distinct \\
\hline 214 PIK3CA & ENST0000026396' E6 & $-17[\mathrm{chr} 3 \mathrm{C}$ & $\mathrm{C} \rightarrow \mathrm{A}($ homo $)$ & $100 \%(785) \quad[100 \%(497) / 100 \%(288)]$ & & rs2699896 (100 & c. $1060-17 C>A$ & 67/POLY & distinct \\
\hline 215 MEN1 & ENST0000031204: E2 & -16 [chr1 C & C $\rightarrow \mathrm{G}$ (het) & $56 \%(98) \quad[56 \%(96) / 50 \%(2)]$ & & rs509606 (1000 & c. $-23-16 C>G$ & $1 / 5$ POLY & forced, distinct \\
\hline 216 BRAF & ENST0000028860: E18 & $-16[\mathrm{chr} 7 \mathrm{C}$ & $\mathrm{C} \rightarrow \mathrm{T}$ (het) & $82 \%$ (14) $[100 \%(10) / 57 \%(4)]$ & & rs368721021 (C & $c .2128-16 C>T$ & 1/1 POLY & distinct \\
\hline 217 BRCA2 & ENST0000054445: E17 & $-14[\mathrm{chr} 1 \mathrm{C}$ & $\mathrm{T}->\mathrm{C}$ (homo) & $100 \%(469) \quad[100 \%(365) / 100 \%(104)]$ & & rs9534262(100 & c. $7806-14 \mathrm{~T}>\mathrm{C}$ & 85/ POLY & distinct \\
\hline 218 PIK3CA & ENST0000026396' E12 & $-13[\mathrm{chr} 3 \mathrm{C}$ & $T->C$ (het) & $52 \%(267)[57 \%(200) / 41 \%(67)]$ & & rs41273619(10 & c. $1747-13 T>C$ & 0/1 POLY & distinct \\
\hline 219 ATM & ENST0000027861 E24 & $-12 / 1 \mathrm{bp}$ I (Dup) & $A$ (het) & $85 \%(130)[80 \%(89) / 98 \%(41)]$ & & ClinVar ben (ML & c.3403-13dupA & 38/ POLY & distinct \\
\hline 220 JAG1 & ENST0000025495: E6 & $10(765)$ IC & $C \rightarrow T$ (het) & $35 \%(310) \quad[35 \%(144) / 35 \%(166)]$ & $\cdot Y(255$ & COSM 3758428 & c. $765 \mathrm{C}>\mathrm{T}$ & p.T 5/7 POLY & distinct \\
\hline 221 KMT2D & ENST0000030106 E11 & $29(2826) \mathrm{C}$ & C $\rightarrow \mathrm{T}$ (homo) & $100 \%(833) \quad[100 \%(421) / 100 \%(412)]$ & . I(942) & CosM3753294 & $c .2826 C>T$ & p.II $3 / 1$ POLY & distinct \\
\hline 222 ATM & ENST0000027861। E40 & $30(5948) \mathrm{C}$ & A $>$ G (homo) & $100 \%(97)[100 \%(44) / 100 \%(53)]$ & $\mathrm{S}(198$ & Ess659243 (1000 & c. $5948 \mathrm{~A}>\mathrm{G}$ & p.A 272 POLY & distinct \\
\hline 223 SMARCA2 & ENST0000034972: E2 & 32 [chr9:! C & $\mathrm{G} \rightarrow \mathrm{A}$ (het) & $58 \%$ (199) $[59 \%(127) / 58 \%(72)]$ & $\therefore$ UTR & rs10964468 (10 & c. $-5 \mathrm{G}>\mathrm{A}$ & 0/3 POLY & distinct \\
\hline 224 KRAS & ENST0000025607: E5 & $33(483)$ IC & $\mathrm{G} \rightarrow \mathrm{A}($ homo) & $100 \%(947) \quad[100 \%(422) / 100 \%(525)]$ & $\cdot R(161$ & rs4362222 (100 & c. $483 \mathrm{G}>\mathrm{A}$ & p.A 79/ POLY & distinct \\
\hline 225 APC & ENST00000257431 E12 & $50(1458) \mathrm{C}$ & $\mathrm{T} \rightarrow \mathrm{C}$ (homo) & $100 \%(723) \quad[100 \%(337) / 100 \%(386)]$ & $\cdot Y(486$ & $\cos 1432175$ & c. $1458 \mathrm{~T}>\mathrm{C}$ & p.T $3 / 2$ POLY & distinct \\
\hline 226 LMNA & ENST0000036830। E5 & $51(861)$ IC & $\mathrm{T}->\mathrm{C}$ (het) & $32 \%(339) \quad[30 \%(143) / 34 \%(196)]$ & - A (287 & rs538089 (1000 & c. $861 \mathrm{~T}>\mathrm{C}$ & p.A 2/8 POLY & distinct \\
\hline 227 FGFR2 & ENST0000035848 E6 & $72(696)$ IC & $A \rightarrow G($ homo $)$ & $100 \%(1149) \quad[100 \%(490) / 100 \%(659)]$ & $\cdot v(232$ & rs1047100 (100 & c. $696 \mathrm{~A}>\mathrm{G}$ & p.V 0/1 POLY & distinct \\
\hline 228 FGFR2 & ENST0000047885! E4 & 94 [chr10 C & $A \rightarrow G($ homo $)$ & $100 \%(668)[100 \%(466) / 100 \%(202)]$ & i UTR & rs2981437(100 & c. $-109 A>G$ & $1 / 0 \mathrm{POLY}$ & distinct \\
\hline 229 KMT2D & ENST0000030106 E39 & $96(10836) \mathrm{C}$ & $\mathrm{G}>\mathrm{A}$ (homo) & $100 \%(827) \quad[100 \%(365) / 100 \%(462)]$ & Q (361 & : COSM431202 (6 & c. $10836 \mathrm{G}>\mathrm{A}$ & p.৫3/1 POLY & distinct \\
\hline 230 SMAD3 & ENST0000032736 E2 & $103(309) \mathrm{C}$ & $A \rightarrow G($ homo $)$ & $100 \%(1004)[100 \%(623) / 100 \%(381)]$ & $\cdot L(103)$ & ) rs1065080 (100 & c. $309 A>G$ & p.L 12/POLY & distinct \\
\hline 231 LMNA & ENST0000036830I E12 & 106 [chr1c & $\mathrm{G}>\mathrm{C}$ (homo) & $100 \%(503) \quad[100 \%(191) / 100 \%(312)]$ & : UTR & rs7339 (1000Ge & c. ${ }^{*} 79 \mathrm{G}>\mathrm{C}$ & 2/7 POLY & distinct \\
\hline 232 MEN1 & ENST0000031204! E9 & $114(1299) \mathrm{C}$ & $\mathrm{T} \rightarrow \mathrm{C}$ (homo) & $100 \%(1269) \quad[100 \%(670) / 100 \%(599)]$ & $\cdot H(433$ & 3 rs540012 (1000 & c.1299T>C & p.t 20/POLY & distinct \\
\hline 233 TP53 & ENST0000026930! E4 & $119(215) \mathrm{C}$ & $\mathrm{C} \rightarrow \mathrm{G}($ homo $)$ & $100 \%(735) \quad[100 \%(313) / 100 \%(422)]$ & $>R(72)$ & $\cos M 3766192$ & c. $215 C>G$ & p.P $14 \subseteq$ POLY & distinct \\
\hline 234 ARID1B & ENST0000034608! E6 & $135(2172 ; C$ & $\mathrm{G}>\mathrm{A}($ homo) & $100 \%(969) \quad[100 \%(377) / 100 \%(592)]$ & - $A(724$ & COSM1487405 & $c .2172 \mathrm{G}>\mathrm{A}$ & p.A 4/1 POLY & distinct \\
\hline 235 BCORL1 & ENST0000054005: E3 & $154(331) \mathrm{C}$ & $\mathrm{T} \rightarrow \mathrm{C}$ (homo) & $100 \%(698) \quad[100 \%(404) / 100 \%(294)]$ & $\cdot L(111$ & ) rs4830173 (100 & $c .331 \mathrm{~T}>\mathrm{C}$ & p.P1/O POLY & distinct \\
\hline 236 KMT2D & ENST0000030106 E10 & $168(1426, \mathrm{C}$ & G ->A (homo) & $98 \%(511) \quad[94 \%(127) / 100 \%(384)]$ & $\cdot \mathrm{T}(476$ & rs1064210 (100 & c. $1426 \mathrm{G}>\mathrm{A}$ & p.A 0/3 POLY & distinct \\
\hline 237 KMT2D & ENST0000030106 E10 & $168(1426, \mathrm{C}$ & $\mathrm{G}>\mathrm{A}($ homo) & $98 \%(511)[94 \%(127) / 100 \%(384)]$ & $\cdot \mathrm{T}(476$ & rs1064210 (100 & c. $1426 \mathrm{G}>\mathrm{A}$ & p.A 0/3 POLY & distinct \\
\hline 238 LMNA & ENST00000368301 E7 & $181(1338, \mathrm{C}$ & $\mathrm{T} \rightarrow \mathrm{C}$ (homo) & $100 \%(216)[100 \%(80) / 100 \%(136)]$ & $\cdot D(446$ & rs505058(1000 & c.1338T>C & p.A 2/8 POLY & distinct \\
\hline 239 LMNA & ENST0000036830I E7 & $181(1338, \mathrm{C}$ & $\mathrm{T} \rightarrow \mathrm{C}$ (homo) & $100 \%(216)[100 \%(80) / 100 \%(136)]$ & $\cdot D(446$ & rs505058 (1000 & c. $1338 \mathrm{~T}>\mathrm{C}$ & p.A 2/8 POLY & distinct \\
\hline 240 SMARCA2 & ENST0000034972: E25 & $216(3672$ C & $\mathrm{G}->\mathrm{A}($ homo) & $100 \%(537) \quad[100 \%(269) / 100 \%(268)]$ & E (1224 & A COSM3763856 & c. $3672 \mathrm{G}>\mathrm{A}$ & p.c $0 / 4$ POLY & distinct \\
\hline 241 JAG1 & ENST0000025495: E26 & $218(3417, \mathrm{C}$ & $\mathrm{T}>\mathrm{C}$ (homo) & $100 \%(866) \quad[100 \%(403) / 100 \%(463)]$ & $Y(1139$ & $9 \cos M 3758426$ & c. $3417 \mathrm{~T}>\mathrm{C}$ & p.T 5/7 POLY & distinct \\
\hline $242 \mathrm{CHD} 1$ & ENST0000028404! E35 & $263 . .265$ (! D & CCT (homo) & $97 \%$ (1467) [97\% (808) / 96\% (659)] & $\cdot(1684$ & $\cos M 327350$ & c.5050_5052delCCT & p.F 0/1 POLY & distinct \\
\hline 243 MEN1 & ENST0000031204! E10 & $271(1621) C$ & A $\rightarrow \mathrm{G}($ homo $)$ & $100 \%(1341) \quad[100 \%(779) / 100 \%(562)]$ & - A (541 & $\operatorname{COSM} 255213$ & c. $1621 \mathrm{~A}>\mathrm{G}$ & p.T 20/POLY & distinct \\
\hline 244 SMAD3 & ENST0000032736 E1 & 296 [chr1C & $\mathrm{G} \rightarrow \mathrm{A}$ (het) & $26 \%(66)[21 \%(38) / 36 \%(28)]$ & i UTR & rs1061427(100 & c. $-15 \mathrm{G}>\mathrm{A}$ & 0/8 POLY & distinct \\
\hline 245 RNF43 & ENST0000040797 E9 & $300(1252 ; C$ & $\mathrm{C} \rightarrow \mathrm{A}($ homo $)$ & $100 \%(2000)[100 \%(1063) / 100 \%(937)]$ & M (418 & 3 COSM4130449 & c. $1252 C>A$ & p.L 13/ POLY & distinct \\
\hline $246 \mathrm{NF} 2$ & ENST0000033864: E1 & $332[\mathrm{chr} 2 \mathrm{C}$ & $\mathrm{G}>\mathrm{C}$ (homo) & $95 \%$ (158) $[95 \%(154) / 100 \%(4)]$ & i' UTR & rs1800540 (100 & c. $-110 \mathrm{G}>\mathrm{C}$ & 12/ POLY & forced, di \\
\hline 247 CASR & ENST0000049013: E7 & $512(2244, C$ & $\mathrm{G} \rightarrow \mathrm{C}$ (homo) & $100 \%(2197) \quad[100 \%(1122) / 100 \%(1075)]$ & )]. P (748 & rs2036400 (100 & c. $2244 \mathrm{G}>\mathrm{C}$ & p.P3/1 POLY & distinct \\
\hline 248 КМт2C & ENST0000026218! E14 & 635 (2448'I I (Dup) & $A$ (het) & $27 \%(195) \quad[29 \%(68) / 27 \%(127)]$ & $816(\mathrm{R}$ & rs150073007 (d & c.2447dupA & p.T 0/1 POLY & distinct \\
\hline 249 KMT2D & ENST0000030106 E31 & $1245(747: C$ & $\mathrm{G}>\mathrm{T}$ (homo) & $100 \%(1035) \quad[100 \%(679) / 99 \%(356)]$ & $G(249$ & COSM3722550 & c. $7479 \mathrm{G}>\mathrm{T}$ & p.৫ $3 / 1$ POLY & distinct \\
\hline 250 KMT2D & ENST0000030106 E31 & $1245(747: C$ & $\mathrm{G}>\mathrm{T}$ (homo) & $100 \%(1035) \quad[100 \%(679) / 99 \%(356)]$ & $G(249$ & COSM3722550 & c.7479G $>\mathrm{T}$ & p.৫3/1 POLY & distinct \\
\hline 251 CASR & ENST0000049013: E7 & $1299(303: C$ & $\mathrm{G}>\mathrm{C}($ homo $)$ & $100 \%(1268) \quad[100 \%(577) / 100 \%(691)]$ & $Q(101$ & 1 rs1801726 (100 & c. $3031 \mathrm{G}>\mathrm{C}$ & p.৫3/1 POLY & distinct \\
\hline 252 CASR & ENST0000049013: E7 & 1565 [chr C & $\mathrm{A} \rightarrow \mathrm{T}($ homo) & $100 \%(623) \quad[100 \%(159) / 100 \%(464)]$ & : UTR & rs4677948 (100 & c. ${ }^{*} 60 \mathrm{~A}>\mathrm{T}$ & 3/1 POLY & distinct \\
\hline 253 BRCA2 & ENST0000054445! E11 & $2654(456 . \mathrm{C}$ & A $\rightarrow G($ homo $)$ & $100 \%(544) \quad[100 \%(280) / 100 \%(264)]$ & $\mathrm{L}(1521$ & 1 rs206075 (1000 & c. $4563 \mathrm{~A}>\mathrm{G}$ & p.L65i POLY & distinct \\
\hline 254 SETD2 & ENST0000040979: E3 & $3378(346 !$ C & $\mathrm{T} \rightarrow \mathrm{C}$ (homo) & $100 \%(1061) \quad[100 \%(579) / 100 \%(482)]$ & $N(115$ & ?rs6767907 (100 & c.3465T>C & p.A $1 / 6$ POLY & distinct \\
\hline $255 \mathrm{APC}$ & ENST00000257431 E16 & 3507 (546! C & $\mathrm{T} \rightarrow \mathrm{A}$ (homo) & $100 \%(345)[100 \%(152) / 100 \%(193)]$ & $\mathrm{D}(182$ & ¿COSM3760871 & c.5465T>A & p.V 4/1 POLY & distinct \\
\hline 256 BRCA2 & ENST0000054445! E11 & 4604 (651: C & $\mathrm{G} \rightarrow \mathrm{C}$ (homo) & $100 \%(335) \quad[100 \%(214) / 100 \%(121)]$ & $V(217$ & 1 COSM4147689 & c. $6513 \mathrm{G}>\mathrm{C}$ & p.l 301 POLY & distinct \\
\hline 257 CASR & ENST0000049013: E7 & $+15 / 1 \mathrm{bp}$ I (Dup) & A (het) & $29 \%(22)[0 \%(0) / 29 \%(22)]$ & & rs33974189 (Mı & c. ${ }^{* 188 d u p A}$ & $0 / 2$ POLY & forced,dist \\
\hline 258 LMNA & ENST0000036830I E6 & +16 [chr1 C & $\mathrm{G} \rightarrow \mathrm{A}$ (het) & $37 \%(422) \quad[35 \%(184) / 38 \%(238)]$ & & rs534807 (1000 & c. $1157+16 G>A$ & 2/8 POLY & distinct \\
\hline 259 LMNA & ENST0000036830। E6 & $+16[\mathrm{chr} 1 \mathrm{C}$ & $\mathrm{G} \rightarrow \mathrm{A}$ (het) & $37 \%(422)[35 \%(184) / 38 \%(238)]$ & & rs534807(1000 & c. $1157+16 G>A$ & 2/8 POLY & distinct \\
\hline 260 CASR & ENST0000049013: E3 & $+19[\mathrm{chr} 3 \mathrm{C}$ & $\mathrm{G} \rightarrow \mathrm{A}($ homo) & $99 \%$ (373) $[100 \%(10) / 99 \%(363)]$ & & rs9869985 (100 & c. $492+19 G>A$ & 3/1 POLY & forced, distinct \\
\hline 261 TP53 & ENST0000026930! E2 & $+38[\mathrm{chr} 1 \mathrm{C}$ & $C \rightarrow G$ (het) & $93 \%(240)[61 \%(30) / 100 \%(210)]$ & & rs1642785 (100 & c. $74+38 \mathrm{C}>\mathrm{G}$ & 11 i POLY & distinct \\
\hline 262 BRCA1 & ENST0000035765، E1 & $+101[\mathrm{chr} C$ & C $>$ G (homo) & $99 \%(307) \quad[100 \%(230) / 99 \%(77)]$ & & rs799905 (1000 & c. $-20+101 C>G$ & $1 / 0$ POLY & distinct \\
\hline 263 KDM6A & ENST0000037796: E18 & $-5 / 1 b p[D$ & $T$ (het) & $45 \%(35)[42 \%(16) / 48 \%(19)]$ & & ExAC, ClinVar (I & c.2703-5delT & 0/1 POLY (s & distinct \\
\hline 264 RNF43 & ENST0000040797 E2 & $524(139) \mathrm{C}$ & $A \rightarrow G$ (het) & $22 \%(396) \quad[22 \%(188) / 21 \%(208)]$ & $>V(47)$ & rs3744093 (100 & $c .139 A>G$ & p.ll 14/Poly & distinct \\
\hline 265 SMARCA2 & ENST0000034972: E4 & $350 . .352$ ( D & GCA (het) & $36 \%(102)[36 \%(46) / 36 \%(56)]$ & $2(23$ & 236) & c.705_707delGCA & p.co/4 Se & \\
\hline 266 BRCA2 & ENST0000054445! E14 & 390 (7397, C & $\mathrm{T} \rightarrow \mathrm{C}$ (homo) & $100 \%(282)[100 \%(91) / 100 \%(191)]$ & A $(2466$ & Ers169547 (1000 & c. $7397 \mathrm{~T}>\mathrm{C}$ & p.V 302 Wildtyp & istinct \\
\hline
\end{tabular}




\section{DISCUSSION}

To increase the success rate of clinical trials in the field of oncology, it is necessary to direct cancer research towards personalized medicine and precision oncology characterized by tailor-made treatments for individual patients [3]. In this study we investigated whether HSP90 clients such as MIF (macrophage migration inhibitory factor) and mutp53 (mutant p53) can serve as potential biomarkers and drug targets in colorectal cancer (CRC) or pancreatic ductal adenocarcinoma (PDAC):

(I) Figures labeled with 'Publication' can be found in the published paper (section 3.1): Klemke et. al. 2021, Cell Death \& Disease [234].

(II) Figures labeled with 'Manuscript' can be found in the provisionally accepted but not yet published manuscript (section 3.2), Klemke et. al. 2021, Frontiers in Oncology.

(III) Figures without any of the mentioned specifications can be found in the dissertation itself.

The results gained in these two subprojects, will be discussed in detail in the following sections.

\subsection{MIF PROMOTES COLORECTAL CANCER PROGRESSION}

Various efforts have been made to better understand the biological function of MIF. It was first discovered as a pro-inflammatory cytokine shown to inhibit random migration of immune cells [116]. However, as research progressed it became evident that it has a more complex biology than previously described. To date, MIF has not just been extensively described as a chemokine but also as a hormone and enzyme [159, 162]. Furthermore, with its pleiotropic functions it can regulate tumorigenic pathways by supporting all hallmarks of cancer [73, 235].

In our current CRC study (Publication in section 3.1) using the colitis-associated AOM/DSS mouse model, we investigated the role of MIF in CRC development. We showed that Mif levels are elevated in CRC cells and that a depletion of Mif results in decreased tumor burden (Publication Figures 1, 2 and S1). These findings are in line 
with results gained in small intestinal tumors of $A p c^{\min }$ mice [149] and xenograft mouse models [111, 151] further supporting MIF as a tumor promoting protein in intestinal cancers.

To understand the core mechanism leading to altered tumor development, we were intrigued to answer the following questions:

(I) Does MIF already affect CRC development during tumor initiation?

(II) Is the tumorigenic effect of MIF limited to established tumors?

Interestingly, we found that MIF is an important player in both phases of CRC tumor development.

To explore the underlying tumorigenic mechanism that could explain the decreased tumor burden after Mif depletion, established tumors were biopsied (Publication Figure $1 \mathrm{~A}$ ) and recovering tissue was prepared to investigate tumor initiation (Publication Figure 3A). The recovery group (representing tumor initiation), is characterized by DSS induced disruption of the intestinal epithelial barrier, causing massive infiltration of immune cells and disruption of the tissue (Publication Figure 3B; section 2.2.1). Within this phase of tumor development, we hypothesized that MIF promotes DSS-induced inflammation as a pro-inflammatory cytokine and Mif loss results in decreased inflammation and better tissue recovery. Therefore, we evaluated the infiltration of regulatory $\mathrm{T}$-cells $\left(\mathrm{FoxP}^{+}\right)$, neutrophil granulocytes $\left(\mathrm{MPO}^{+}\right)$and $\mathrm{T}$ lymphocytes $\left(\mathrm{CD}^{+}\right)$into the damaged tissue.

During initial stages, MIF acted as a pro-inflammatory cytokine on overall inflammation, particularly on the recruitment of regulatory T-cells, neutrophil granulocytes and Tlymphocytes (Publication Figures 3B-D and S2A-C). In contrast, this effect was not observed in established tumors (Publication Figures 4A and S3A, B). Interestingly, monocyte/macrophage infiltration was altered in established tumors but not in adjacent normal epithelium (Publication Figure 4B) or during tumor initiation ('recovery'; Publication Figures $3 \mathrm{C}$ and S2A). Furthermore, angiogenic (CD31, VEGF) and proliferative (Ki67) markers were specifically regulated in established tumors after Mifdepletion (Publication Figures $4 \mathrm{C}-\mathrm{H}$ ) but not during tumor initiation (Publication Figure S3G-K). Our in vitro (HCT116 and DLD-1; Publication Figures 5A-G) and ex vivo (Mif-depleted colon-derived tumor organoids; Publication Figures 6F, G) studies 
confirm involvement of tumor cell-derived MIF in angiogenic pathways. Together with clinical patient correlation studies (Publication Figure $\mathbf{5 H}-\mathbf{J}$ ), these data showed that MIF acts in a CD74-dependent manner in CRC. Using matched pairs of colonic organoids (normal and tumor tissues from the same donor), we confirmed MIF as an important HSP90 client and demonstrated that stabilized MIF results in higher susceptibility of tumor cells towards HSP90 inhibition (Publication Figures 6H-I, 7AD, S5B, C).

Taken together, these data suggest that MIF function switches from a pro-inflammatory cytokine during tumor initiation to a HSP90-stabilized protein driving tumorigenic mechanisms in established tumors (Figure 8).

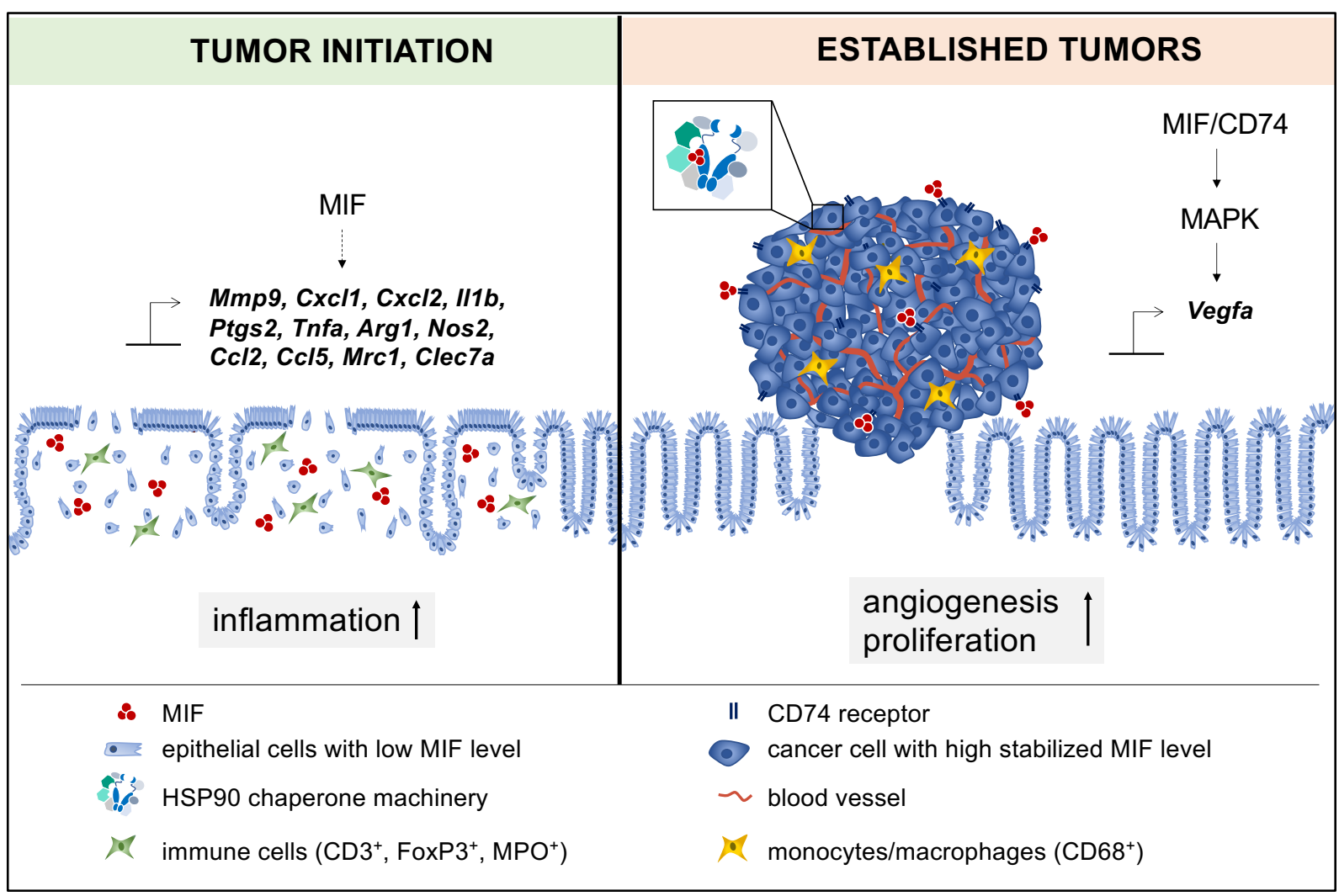

Figure 8: Functional switch of MIF during colorectal cancer progression. During tumor initiation MIF functions as a pro-inflammatory cytokine to regulate overall inflammation via recruitment of $\mathrm{CD}^{+}, \mathrm{FoxP}^{+}, \mathrm{MPO}^{+}$immune cells and cytokine expression. In contrast, in established tumors MIF regulates the recruitment of tumor-associated macrophages and is involved in tumorigenic pathways such as proliferation and angiogenesis. Notably, MIF no longer regulates overall inflammation in established tumors. 


\subsubsection{MIF contributes to angiogenesis, but only in established tumors - a CD74- dependent mechanism}

MIF has been described to promote colorectal tumorigenesis through regulation of angiogenesis [111, 149, 151, 236]. In established tumors of Mif-deficient mice, angiogenic markers (CD31 and Vegf) were downregulated compared to Mif-proficient mice (Publication Figures 4C-G). Furthermore, infiltration of tumor associated macrophages was reduced after Mif loss (Publication Figure 4B). From our in vivo study, we cannot conclude whether the MIF-dependent angiogenic effects are due to receptor mediated pathways in cancer cells themselves (Figure 5) or are a result of recruited tumor-associated macrophages, which are known to express and secrete angiogenic factors such as VEGF to support tumor progression [167, 170].

In this context, three different scenarios would be possible (Figure 9):

(I) Cancer cells harbor high levels of HSP90-stabilized MIF which is secreted into the surrounding stroma. The secreted MIF can act in an autocrine manner via the CD74 receptor to increase the expression and secretion of angiogenic factors such as VEGF and IL-8 from cancer cells [109, 111, 151, 155, 159].

(II) In a paracrine fashion, cancer cell-derived MIF also acts on constituents of the stromal compartment. MIF/CD74 interaction has been shown to be particularly important for chemotaxis of monocytes/macrophages [237]. In addition, tumor cell-derived MIF has been reported to contribute to the angiogenic potential of macrophages by inducing the expression of factors such as VEGF $[161,167$, 238, 239]. Furthermore, tumor cell-derived MIF can promote neovascularization, by direct interaction with endothelial cells, resulting in their proliferation, migration and tube formation [162, 239].

(III) Stromal cells are also known to express and secrete MIF, which can act in an autocrine or paracrine fashion, further contribute to the expression of angiogenic factors [117, 167, 239-241]. Thus, the secretion of MIF from stromal cells can also contribute to neovascularization of tumors [167, 242].

In a tumor bulk, which consists of epithelial cancer cells and constituents of the tumor microenvironment, any combination of the three scenarios might contribute to vessel formation [168, 239]. However, scenarios (I) and (II) are most likely, since tumor cells 
have high levels of stabilized MIF, hence being the major source of secreted MIF [57, 111].

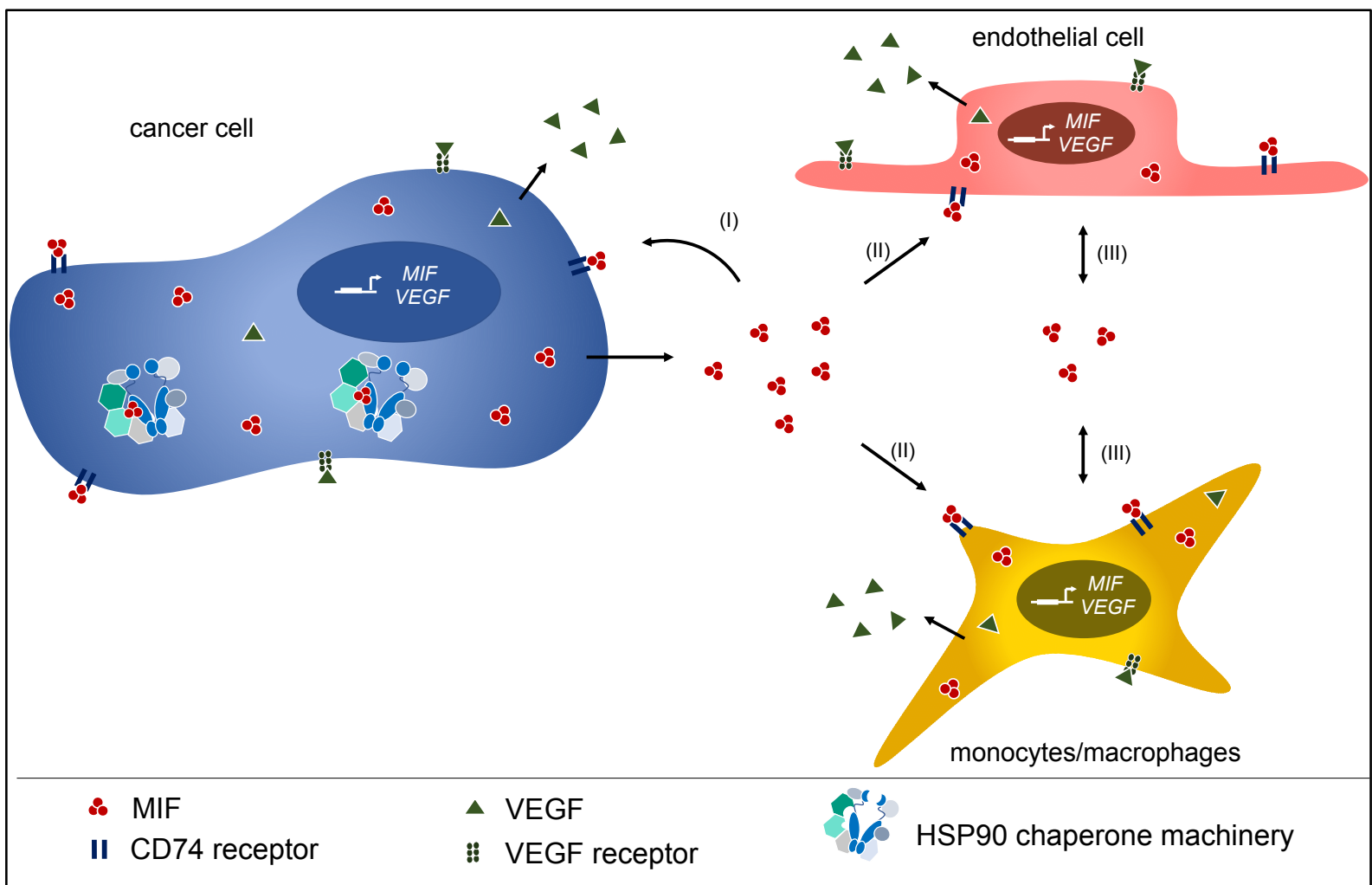

Figure 9: Possible scenarios on how MIF triggers angiogenesis in cancer. In cancer cells, MIF is highly stabilized by the HSP90 superchaperone machinery resulting in elevated MIF levels in cancer cells. (I) Secreted MIF acts in an autocrine loop, by binding to the CD74 receptor on cancer cells, promoting tumorigenesis via inducing expression and secretion of angiogenic factors (VEGF). (II) Moreover, tumor cell-derived MIF can act in a paracrine manner on monocytes/macrophages and endothelial cells which further promotes angiogenesis. (III) Macrophages/monocytes and endothelial cells also express and secrete MIF, which additionally triggers angiogenic responses by paracrine and autocrine loops.

Colorectal cancer cells have elevated MIF levels due to the stabilization via the HSP90 chaperone machinery (Publication Figures 2, 6 and 7; Figure 5), suggesting that most of the secreted MIF arises from these tumor cells themselves and contributes to angiogenesis. To prove this hypothesis, we used epithelial cell cultures to evaluate the expression of angiogenic factors. In Mif-deficient tumor organoids (Publication Figure 6G) as well as in human cancer cells (HCT116) (Publication Figure 5B), we found reduced expression of VEGFA or IL8 after MIF loss, suggesting that HSP90-stabilized MIF in tumor cells themselves contributes to neovascularization in CRC via an autocrine loop. Of note, this effect was only observed in the presence of the cognate CD74 receptor (Publication Figures 5A-G). 
Interestingly, we found that macrophage recruitment was specifically altered in established tumors, but not in adjacent normal tissue (Publication Figure 4B). MIF has been suggested to drive recruitment and activation of tumor-associated macrophages in a CD74-dependent manner, thereby promoting tumor progression via immunosuppression and angiogenesis [167, 168]. In line with these findings, Fan et al. demonstrated that Mif- and CD74-deficient murine macrophages displayed diminished chemotaxis [237]. The reduced chemotactic potential of macrophages in the absence of MIF might explain the lower number of macrophages observed in Mifdepleted tumors in vivo (Publication Figure 4B). In contrast to macrophages, other tested immune cells (neutrophil granulocytes, T-lymphocytes or regulatory T-cells) were not regulated in established tumors of Mif-deficient mice compared to wildtype mice. These immune cells are also known to contribute to tumor progression and to be regulated by MIF [161]. Indeed, the recruitment of T-cells [128, 243] and neutrophils $[244,245]$ has been mainly reported via the receptors CXCR2 and CXCR4. Taken together, the regulation of tumor associated macrophages but not neutrophil granulocytes, T-lymphocytes or regulatory T-cells (Publication Figures 4A, B and S3A), confirms the involvement of the MIF/CD74 axis in established CRC.

Further experiments are necessary to further investigate whether MIF in tumor cells or stromal cells (e.g., macrophages, fibroblasts, endothelial cells) constitutes the driving force of tumor progression. In that context, Hu et al. demonstrated that human hepatic sinusoidal endothelial cells (HHSECs) as well as human umbilical vein endothelial cells (HUVECs) cells were able to induce chemotaxis and migration in different CRC cell lines (SW480, HCT116) via secretion of MIF [246]. Vice versa, their study also showed, that MIF secreted from these cancer cells did not induce migration of endothelial cells [246]. In contrast, boyden chamber assays using HUVECs treated with recombinant MIF confirm its important role on neovascularization [241]. To further elaborate on the communication between cancer and endothelial cells, one could perform transwell and boyden chamber assays (with HUVECs) using the supernatant of MIF-proficient and MIF-depleted human epithelial cancer cells (e.g., HCT116) or matched pairs normal and tumor organoids from CRC patients.

However, additional preclinical proof-of-principal studies would help to understand the importance of cancer-derived MIF in the context of tumor development. Therefore, Miffl/fl; ;illincre mice can be used to specifically deplete MIF from colon epithelial cells, 
while MIF expression in the tumor stroma remains unaltered [247]. These transgenic mice are characterized by the constitutive expression of the cre recombinase under regulatory control of the villin promotor [247]. The cre recombinase specifically recognizes loxP (floxed allele, fl) sequences allowing the recombination of defined target genes [247]. In Mif//ff; ;illincre mice, the Mif gene is flanked with two loxP sites, allowing the villincre recombinase to deplete Mif from intestinal epithelial cells.

By using this mouse strain in comparison to our constitutive Mif knockout mouse used in the current study, the following results would be expected:

(I) If MIF in cancer cells is the driving force of CRC development, an epithelialspecific depletion of MIF would result in decreased tumor burden.

(II) If MIF in stromal cells is the most important source for CRC development, we would not see any alteration of tumor growth after MIF loss in epithelial cells.

(III) If a combination of MIF in cancer and stromal cells results in the development of colorectal tumors, a milder reduction in tumor burden would be seen compared to that observed in the overall constitutive Mif knockout mice.

Evaluation of tumor burden, tumorigenic pathways and macrophage recruitment can reveal whether depletion of HSP90-stabilized MIF specifically in the intestinal epithelial cancer cells is the driver of tumor development in CRC.

An additional step would be to use a conditional Mif knockout mouse (Mif ${ }^{f / f}$; villincreERT2). In these mice, the cre recombinase is fused with the mutated ligand-binding domain of the estrogen receptor ERT2 which interacts with HSP90 [247, 248]. Upon treatment with Tamoxifen HSP90 dissociates and the creERT2 fusion protein translocates into the nucleus to induce recombination of the target gene of interest [248]. This mouse model allows the depletion of genes at a defined time point. For the current study it would be of interest to use these conditional mice to investigate whether tumor cell-derived MIF is important for tumor maintenance. For those experiments, mice would be treated with AOM/DSS to induce tumor development. Then, Mif recombination is initiated using Tamoxifen at a particular tumor size. These approaches are especially important for clinical translation, since CRC patients are often diagnosed at a later stage of the disease $[107,108]$. Therefore, most of these patients would already have established CRC tumors at the start of their treatment 
[107]. Hence, the Mifflff; $v$ illincreERT2 mice would be a suitable model to investigate if MIF is essential for CRC maintenance and can serve as a suitable biomarker for treatment in the clinic.

Furthermore, these mice provide the possibility of co-cultures between Mif-proficient and Mif-deficient colonic tumor organoids together with isolated peritoneal Mifproficient macrophages. These approaches enable the analysis of macrophage recruitment and activation dependent on the MIF status of the tumor cell. A few other groups have recently started using Mif//fl mice to investigate effects of tissue-specific Mif-depletion on renal inflammation [249] or nonmelanoma skin cancer development [250]. Thus far, in vivo data from a mouse model for colorectal cancer remain elusive, which raises the need for further research.

To better understand whether tumor cell-derived MIF plays a role in mediating tumorigenesis, we investigated the underlying molecular mechanism by which MIF contributes to angiogenesis and proliferation. Analysis of ERK and p38 activity in Mifdeficient tumors revealed decreased phosphorylation of both MAPKs (Publication Figure 4F). Both factors are downstream of MIF's cognate CD74 receptor and are involved in proliferation and angiogenesis [127, 134, 159], which is supported by decreased VEGF levels after Mif loss in vivo (Publication Figures 4C, F, G; Figure 5). Concomitantly, in human CRC cells (HCT116, RKO), the regulation of ERK activity and downstream angiogenic genes were dependent on the presence of MIF and its cognate receptor CD74 (Publication Figures 5A-G). Accordingly, human patient correlation studies revealed that high levels of MIF in CRC cells along with high levels of CD74 correlated with reduced overall survival of patients compared to high MIF levels alone (Publication Figures $5 \mathbf{H}, \mathrm{I}$ ). In contrast, presence of CD74 in the absence of high MIF levels (MIFlow) did not impact patient survival (Publication Figure $5 \mathbf{J}$ ). Taken together, these findings suggest that MIF acts on angiogenesis and proliferation in a CD74-dependent manner in CRC. Research from other groups confirm the importance of MIF and CD74 interaction in tumor progression throughout different cancer origins [157, 251, 252].

In sum, we found that HSP90-stabilized Mif in established colorectal cancer cells acts in a CD74-dependent manner, contributing to angiogenesis via regulation of MAPKs ERK and p38. 


\subsubsection{MIF contributes to overall inflammation, but only during tumor initiation - a CD74-independent mechanism?}

We next aimed to investigate, whether MIF is already important for tumor initiation ('recovery'). Interestingly, angiogenic and proliferative factors, which were regulated in a CD74-dependent manner in established tumors (section 4.1.1) remained unchanged in $\mathrm{Mif}^{-}$- mice during tumor initiation (Publication Figures S3G-K) which hints towards CD74-independent mechanisms of MIF. Indeed, the complexity of MIF receptors is evolving, since CD74/CD44 and non-cognate receptors (CXCR2, CXCR4, CXCR7) can signal individually, but have also been reported to form heterodimers [124, 128, 130, 159] (section 2.2.2 and 2.2.3).

Following DSS administration, massive tissue damage was observed in all animals of the recovery group; this effect was even more pronounced in Mif-proficient than Mifdeficient mice (Publication Figure 3B). We hypothesized that MIF loss might result in better recovery of the tissue due to decreased inflammatory processes, which led us to investigate some major players of the inflammatory pool. A variety of different hematopoietic cells such as neutrophils, macrophages and T-cells are essential drivers of a rapid wound healing response [253]. Neutrophils (MPO ${ }^{+}$) are the main immune cell population in human blood protecting the host from various infections [245, 254]. Bacterial infiltration into the tissue (e.g., by DSS administration, section 2.2.1), causes massive infiltration of neutrophils into the damaged area [245]. However, the exact role of neutrophils has been controversially discussed and is highly context dependent [254]. Besides their role in promoting tissue recovery, neutrophils act against the infiltrated microbiome by secreting proteases and reactive oxygen species (ROS) which might also contribute to further damage of host tissue and might result in delayed regeneration $[245,255]$. T-Lymphocytes $\left(C D 3^{+}\right)$are known to be potentially harmful for tissue recovery [256]. They secrete pro-inflammatory cytokines such as TNF $\alpha$, which can trigger inflammatory responses and decrease tissue regeneration [256, 257]. Regulatory T-cells (FoxP3 ${ }^{+}$) are also important drivers of inflammation and tissue repair by modulating the infiltration of other immune cells such as neutrophils and $\mathrm{T}$ lymphocytes [256]. Last but not least, macrophages have also been shown to play an important role in tissue repair and regeneration by secreting chemokines and matrix metalloproteinases [253]. 
Indeed, we found overall inflammation to be downregulated in Mif-deficient mice during tumor initiation (Publication Figures 3C, D and S2A-C). Importantly, T-cell and neutrophil recruitment were altered upon Mif loss, while recruitment of CD68 positive monocytes/macrophages remained unchanged (Publication Figures $3 \mathrm{C}$ and S2A). As it has been reported that Mif and CD74 deficient murine macrophages display diminished chemotaxis [237], it was interesting to see that Mif loss did not alter macrophage recruitment during tumor initiation. In contrast, neutrophils which have so far been reported in the literature to be CD74 negative [133, 258, 259] showed diminished infiltration in the absence of Mif.

These data further suggest that CD74-independent mechanisms could be involved in the recruitment of immune cells during tumor initiation. Notably, the MIF-mediated recruitment of T-cells and neutrophils have been reported to occur mainly via CXCR2 and CXCR4 [124, 128, 161, 162, 243]. These results hint towards the binding of MIF to these receptors, to drive overall inflammation during tumor initiation. In accordance, Farr et al. reported that CD74 loss in vivo results in increased inflammation-induced tissue damage, caused by decreased tissue recovery [260]. One reasonable explanation for this observation could be the shift of MIF towards interaction with its non-cognate receptors CXCR2 and CXCR4 [128, 243, 260]. Consequently, due to the co-regulation of receptors and the possible shift of ligands/receptor binding, knockout or overexpression experiments in this context might not be ideal. Though out of scope of our current study, it would be of importance to understand the dynamics of these MIF receptors to further understand the role of MIF during CRC initiation.

Taken together we deduce that the reduction of tumor burden in Mif/- mice might be a combination of: (I) decreased inflammation and better recovery during tumor initiation and (II) diminished proliferation and angiogenesis in established tumors.

\subsubsection{HSP90-stabilized Mif contributes to tumor cell survival}

Next, we aimed to investigate how Mif levels are elevated in colorectal cancer cells and whether it can serve as a drug target in CRC. In established tumors, MIF levels were upregulated at mRNA level (approx. 2.5-fold in mouse and human samples; Publication Figures 2A, F and S1A, C), which could be due to increased activation of its transcription factor HIF1 $\alpha[155,261]$ (Figure 5). However, using immunoblot 
analysis, we found that MIF was even more strongly upregulated on protein levels (up to 5.1-fold in mouse and up to 8.5-fold in human samples; Publication Figures 2B, E and S1B). We previously showed that this protein elevation is due to increased stabilization of Mif through the HSP90 chaperone machinery in a mouse model for breast cancer [57, 76]. In our current study, we confirm elevation of Mif in CRC cells to be driven by stabilization via HSP90 (Publication Figures 6 and 7), thus raising the question:

Is MIF also a targetable, cancer-relevant HSP90 client in colorectal cancer?

To answer this question, mice were treated with the HSP90 inhibitor 17AAG to assess tumor burden. Interestingly, reduction of tumor growth was more pronounced in Mifproficient than Mif-deficient mice (Publication Figures 6B-E), suggesting MIF as an important HSP90 client in CRC. In line with these results, Mif-depleted colonic tumor organoids were less susceptible towards HSP90 inhibitor treatment (17AAG) than Mifproficient tumor organoids (Publication Figures 6G-I). Furthermore, we used matched pairs of colonic organoids (normal and tumor material derived from the same mouse), allowing us to investigate tumor-specificity and toxicities of HSP90 inhibitors ex vivo. Importantly, tumor-derived organoids, containing stabilized Mif, showed increased death after HSP90 inhibitor treatment compared to matched pairs of normal epithelial-derived organoids, containing unstabilized Mif (Publication Figures 7A-D). Even though immunoblot analysis reveals degraded Mif in both normal- and tumorderived organoids, only degradation of tumor-derived, stabilized Mif resulted in increased organoid death. Again, these results confirm tumor promoting functions of MIF due to epithelial-specific stabilization through the HSP90 chaperone machinery.

Taken together, we propose that the combination of novel HSP90 inhibitors (reducing HSF1 response, section 2.1.3) together with anti-MIF agents might provide a possible way to selectively target tumor cells and increase anti-tumor effects. Furthermore, the combination treatments could provide a mechanism to bypass cancer resistance [19, 80].

In that respect, inhibition of MIF function can be achieved by using small molecules or antibodies against MIF itself or its cognate receptor CD74 [159, 175]. Small molecules against the tautomerase domain of MIF like ISO-1 have been extensively studied in preclinical trials for different cancers [175, 262, 263]. However, clinical trials were mostly conducted using antibodies against MIF or its cognate receptor CD74 [175]. 
Early phase clinical trials using BAX69 (Imalumab, anti-MIF) or Milatuzumab (hLL1, anti-CD74) showed low toxicity; however, efficacy needs to be further assessed [175, 264, 265]. BAX69 is an antibody particularly raised against oxidized MIF (oxMIF) which has been studied in clinical and preclinical cancer trials thus far [175, 265, 266]. To date, very little is known about the redox-dependent isoform of MIF and its biological properties and more research is needed in this field [267]. However, it has been reported that oxMIF might be the disease related isoform which has been shown to be upregulated in tumor cells of CRC patients [265, 266]. Thiele et al. proposed that oxidation of MIF results in a conformational change of the protein [268]. Based on these data, it could be speculated that the conformational change of MIF due its oxidation increases its dependency on HSP90 superchaperone binding, resulting in stabilization of MIF in colorectal cancer cells. As most other studies in this research area, we did not distinguish between reduced and oxidized MIF. Consequently, it would be of interest to differentiate the two and to find out if oxMIF is the isoform that is more dependent on the HSP90 superchaperone support.

More recently, the use of nanobodies against MIF has been investigated in the context of endotoxic shock [175, 269]. Nanobodies are camelid-derived single-domain antigenbinding antibody fragments $[269,270]$. Compared to conventional monoclonal antibodies, nanobodies have been shown to have better solubility and tissue penetration, as well as lower immunogenicity [269]. Hence, the use of nanobodies might also be of interest for targeting oxidized MIF in preclinical and potential clinical studies in order to increase the efficacy of anti-MIF therapy in colorectal cancer.

In conclusion, post-translational modifications such as oxidation of MIF might provide an explanation for the functional switch of MIF from a pro-inflammatory cytokine during tumor initiation, to a tumor promoting protein in established tumors.

Our study confirms that MIF can serve as a biomarker in colorectal cancer. Due to the lack of successful clinical studies so far, a combination of different cancer treatments might be of interest [265]. We hypothesize that the combination of HSP90 inhibitors and anti-MIF or anti-CD74 therapies could provide a novel approach to target colorectal cancer cells in individual patients. Of note, we have previously shown that mutp53 $3^{\mathrm{R} 248 / \mathrm{W}}$ is also a cancer relevant HSP90 client, driving tumor growth and invasion in CRC [56]. Hence, in those tumors harboring the stabilized mutp53 $2248 \mathrm{Q} / \mathrm{W}$ variant HSP90 inhibition would concomitantly result in a degradation of MIF and 
mutp53 $248 \mathrm{Q} / \mathrm{w}$. This effect might be a promising basis for novel treatment strategies, using biomarkes in order to increase the efficacy of cancer therapy.

\subsection{MUTP53 ${ }^{R 248 Q / W}$ PROMOTES MIGRATION IN CRC AND PDAC}

Since decades, researchers from all over the world have been trying to investigate the role of the tumor suppressor p53 in cancer. As 'guardian of the genome', p53 is mutated in a majority of cancers providing a selection advantage for cells [188, 190, 201, 221]. Over time it became evident, that different mutants of p53 must be considered as individual proteins, harboring different functions [204, 213]. The exact biological function of mutp53 variants is dependent on the mutation itself as well as newly arising complex interactions with other proteins [204, 210, 224] (section 2.3.3). In a previous study in colorectal cancer, we showed that the interaction of mutp53R248Q/W with pSTAT3 drives proliferation and invasion of cancer cells [56] (section 2.3.4). Based on these results, we proceeded to investigate the impact of mutp53 $3^{\mathrm{R} 248 \mathrm{~W}}$ in PDAC. A panel of different PDAC cell lines revealed high levels of stabilized mutp53 ${ }^{\mathrm{R} 248 \mathrm{~W}}$ in MIA-PACA-2, mutp53 ${ }^{\mathrm{C} 176 \mathrm{~S}}$ in PA-TU-8902 and mutp53 ${ }^{\mathrm{R} 282 \mathrm{~W}}$ in PA-TU-8988T cells (Manuscript Figure 1A, B). Treatment of PDAC cells with second generation HSP90 inhibitors Ganetespib and Onalespib resulted in reduced mutp53 levels in all cell lines except BXPC-3 cells harboring mutp53 ${ }^{\mathrm{Y} 220 \mathrm{C}}$ (Manuscript Figure 2A). In PANC-1, MIA-PACA-2, PA-TU-8902 and PA-TU-8988T cells, HSP90 inhibitor treatment resulted in reduced cell confluency (Manuscript Figure 2B). These data reveal that all mutants except Y220C are targetable using HSP90 inhibitors.

As we have previously shown that stabilized mutp53 variants bind to PSTAT3 in colorectal cancer cells [56], we aimed to investigate if this also occurred in PDAC. Therefore, we performed co-immunoprecipitations in different PDAC cell lines. Strongest interaction was observed between mutp53 and pSTAT3 in MIA-PACA-2 and PA-TU-8988T cells (Manuscript Figure 4B-D). Of note, the interaction of mutp53 and PSTAT3 was independent of the levels of pSTAT3; even upon PSTAT3 induction in MIA-PACA-2, PANC-1 and BXPC-3 cells, strongest interaction was only observed between mutp53 ${ }^{\text {R248W }}$ (MIA-PACA-2 cells) and pSTAT3 (Manuscript Figure 4H). This confirmed our results in CRC, indicating that specific variants of stabilized mutp53 predict new complex formations to drive tumorigenesis. 
In CRC, mutp53 $248 \mathrm{Q} / \mathrm{w}$ - pSTAT3 complexes drive migration and invasion [56]. To evaluate if p53 mutants also acquire such new and specific functions in PDAC, we depleted single p53 mutants in different PDAC cells. Importantly, a depletion of mutp53 only diminished migration in MIA-PACA-2 cells harboring mutp53 ${ }^{\mathrm{R} 248 \mathrm{~W}}$ (Manuscript Figure 3A, F). It as to why the PA-TU-8988T cells, which showed a strong binding between $\mathrm{p} 53^{\mathrm{R} 282 \mathrm{~W}}$ and $\mathrm{pSTAT} 3$ failed to have a GOF on migration remains speculative. Possible explanations will be discussed in the following sections in more detail.

Furthermore, an ablation of mutp53 only resulted in reduced pSTAT3 levels in MIAPACA-2 cells but not in any other tested cell lines (Manuscript Figure 4E and S2B), suggesting that only the specific mutp53 ${ }^{\mathrm{R} 248 \mathrm{~W}}$ regulates the activity of STAT3. Concomitantly, the absence of pSTAT3 reduced migration of MIA-PACA-2 cells but not PA-TU-8988T or PANC-1 cells (Manuscript Figure 5A-D). Finally, clinical data revealed that R248 missense mutations of p53 decrease overall patient survival compared to other p53 mutations (Manuscript Figure 5E). In sum, these data suggest that mutp53 ${ }^{\mathrm{R} 248 \mathrm{~W}}$ drives migration of PDAC cells via its complex formation with pSTAT3 in a similar manner to that observed in CRC [56] (Figure 10).

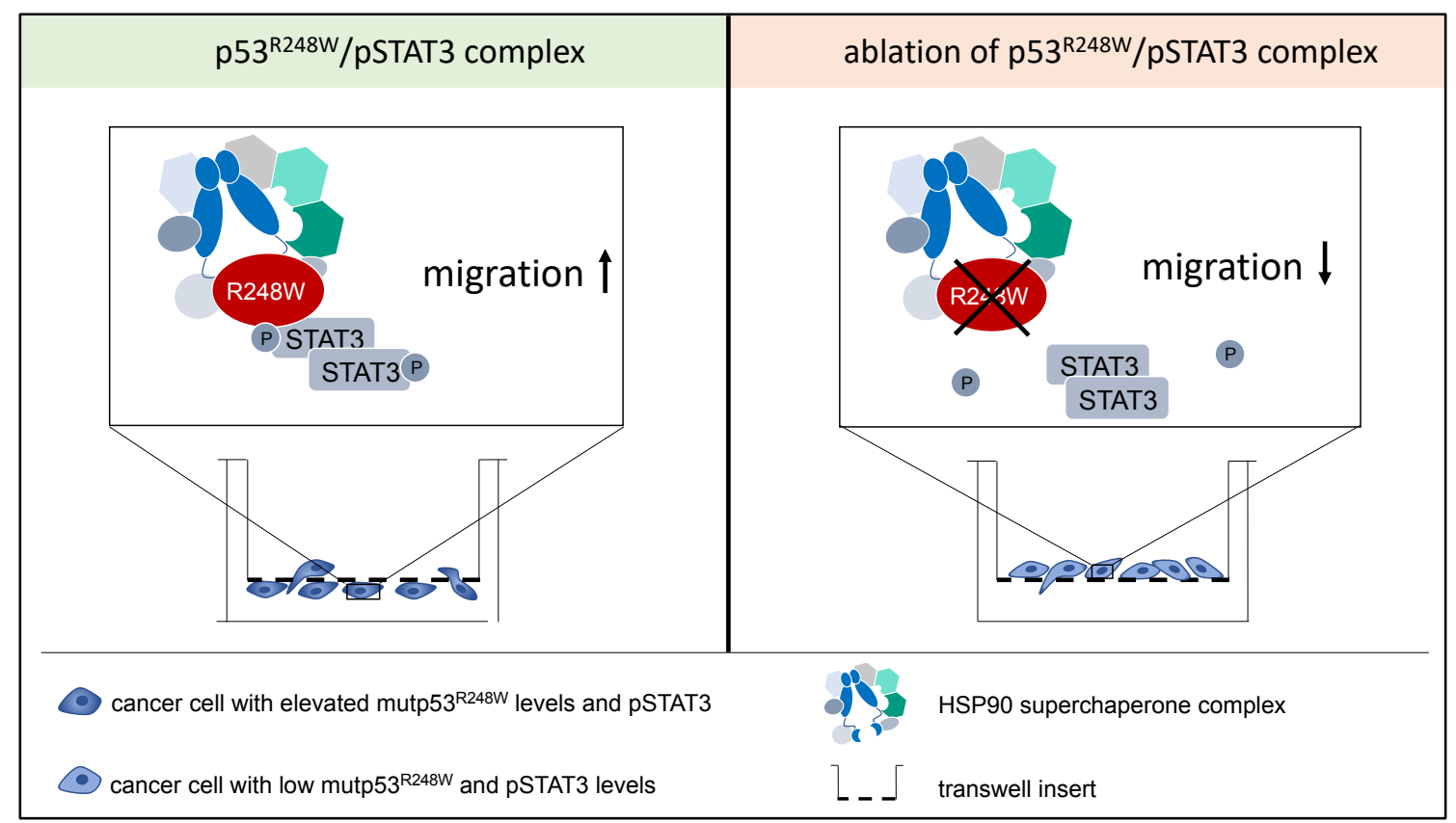

Figure 10: Migratory potential of PDAC cells depends on mutp53 ${ }^{\mathrm{R} 248 \mathrm{~W}}$ and $\mathrm{pSTAT3}$ complex formation. In PDAC cells mutp53 ${ }^{\mathrm{R} 248 \mathrm{~W}}$ is stabilized by the HSP90 superchaperone complex. Depletion of mutp53 ${ }^{\mathrm{R} 248 \mathrm{~W}}$ reduces $\mathrm{pSTAT3}$ levels and results in decreased migration of PDAC cells. Ablation of STAT3 only diminishes migration of mutp $53^{\mathrm{R} 248 \mathrm{~W}}$ harboring cells. 


\subsubsection{Distinct complex formation of mutp53 variants}

The current study confirms the importance of mutp53 $3^{\mathrm{R} 248 \mathrm{~W}}-\mathrm{pSTAT} 3$ complex formation on migration in PDAC and the regulatory impact of mutp53 ${ }^{\mathrm{R} 248 \mathrm{~W}}$ on STAT3 activity, which was already observed in colorectal cancer in vivo and in vitro [56]. To date, the only existing PDAC cell line harboring the R248 mutation of p53 is the MIAPACA-2 cell line. Options to overcome this issue would be:

(I) Altering the endogenous alleles using targeted homologous recombination (e.g., CRISPR/Cas9) to generate R248W mutations in other cell lines. This approach enables the study of proteins under normal regulatory control elements [271, 272].

(II) Generation of isogenic cell lines, by altering the endogenous alleles using homologous recombination. Analyzing different mutation in one genetic cell background helps to exclude effects from other cell-specific mutations and alterations [272, 273].

In colorectal cancer, Sur et al. made use of this approach to study the physiological function of mutp53 ${ }^{\mathrm{R} 248 \mathrm{~W}}$ independent of plasmid overexpression induced effects [271]. Furthermore, CRISPR/Cas9 technology helps to maintain the gene copy number [273], which might be of tremendous importance in the case of stabilized proteins. Because of that, we propose the use of CRISPR/Cas9 or other approaches using homologous recombination technology in isogenic cell line models to better understand the impact of different single nucleotide polymorphisms on the function of the protein under isogenic conditions.

Although our current study shows that the specific variant mutp53 ${ }^{\mathrm{R} 248 \mathrm{~W}}$ regulates PSTAT3 in PDAC, further research is needed to investigate the underlying mechanism. One possible explanation for increased pSTAT3 levels in the presence of mutp53 $248 \mathrm{~W}$ could be the displacement of the phosphatase SHP2, as observed in CRC [56]. However, we cannot exclude that other phosphatases known to regulate STAT3 activity might also be involved [274].

As displayed in section 2.3.3, mutp53 has been described to exert its gain of tumorigenic functions via interaction with other proteins such as the transcription factor 
pSTAT3 [56, 210] (Figure 6). However, the exact underlying mechanism and the interacting proteins are highly dependent on the cellular context [204, 210]. Stabilization of mutp53 through the HSP90 complex is a prerequisite for GOF in cancer cells [56, 221, 222] (Figure 3). The presence of certain co-chaperones or posttranslational modifications of the HSP90 chaperone complex are known to define its interaction with client proteins $[23,75]$. Therefore, different co-chaperones and modifications of the HSP90 superchaperone complex might also have an impact on interaction partners of mutp53 [210]. In the current study, we did not elaborate on any further interaction partners of mutp53 ${ }^{\mathrm{R} 248 \mathrm{~W}}$ in PDAC. Given that many proteins have been described to interact with different mutants of p53 [204, 210, 224, 233], we cannot exclude that other proteins might also be part of the complex (hetero TF complex

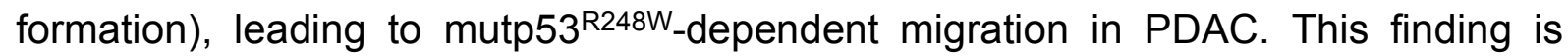
further supported by the fact that STAT3 is reported to form hetero transcription factor (TF) complexes with other proteins such as other STAT isoforms [275], NF- $\kappa$ B [276] or NFATc1 [277] in cancer.

Based on our current study, we cannot state whether mutp $53^{\mathrm{R} 248 \mathrm{~W}}$ binds to pSTAT3 homodimers or hetero TF complexes [277] and/or whether this interaction supports the recruitment of additional co-factors that drive the transcriptional program [278]. However, the formation of hetero TF complexes might provide an explanation for binding of mutp53 to pSTAT3 in various PDAC cells (Manuscript Figures 4B-D), without regulating migration (Manuscript Figure 3A-H). One such example is the PATU-8988T cell line, which showed strong binding of mutp53 $282 \mathrm{~W}$ to pSTAT3 (Manuscript Figure 4D). However, reduced phosphorylation levels upon mutp53 ablation and diminished migration were not observed (Manuscript Figures $3 \mathbf{C}, \mathbf{H}$ and 4E). STAT3 is known to be involved in many of the hallmarks of cancer, such as angiogenesis, proliferation and inflammation to promote tumor progression $[279,280]$. To date, very little is known about the exact interaction partners of mutp53 $282 \mathrm{~W}$. These results again suggest that mutp $53^{\mathrm{R} 282 \mathrm{~W}}$ might be part of a hetero TF complex, together with other proteins and transcription factors, resulting in a gain-of-function other than migration.

Further examples for hetero TF complexes have been proposed for mutp53 $3^{\mathrm{R} 273 \mathrm{H}}$ and mutp53 $3^{\mathrm{R} 175 \mathrm{H}}$. Cooks et al. have previously reported that mutp53 ${ }^{\mathrm{R} 273 \mathrm{H}}$ in PANC-1 cells supports activation of NF- $\kappa \mathrm{B}$ to promote inflammation-associated cancer progression 
[281]. The authors hypothesize that this effect could be due to a direct protein-protein interaction as observed for other mutp53 variants [281]. In this context, mutp53 ${ }^{\mathrm{R} 175 \mathrm{H}}$ and the corresponding murine mutp $53^{\mathrm{R} 172 \mathrm{H}}$ were also reported to enhance NF- $\kappa \mathrm{B}$ activity $[282,283]$. Murine mutp53 ${ }^{R 172 H}$ was shown to physically interact with NF- $\kappa B$ p65 [283] and both homologs were found to colocalize with the transcription factor on some of its promotor sites, regulating the transcriptional outcome [283, 284]. Interestingly, besides strong interaction of mutp53 to pSTAT3 in MIA-PACA-2 cells, we found low binding in PANC-1, BXPC-3 and CAPAN-1 cells (Manuscript Figures 4B, C). One explanation for the minor co-precipitation of pSTAT3 in PANC-1 could be the formation of hetero TF complexes between mutp53 ${ }^{\mathrm{R} 273 \mathrm{H}}$ and $\mathrm{NF}-\kappa \mathrm{B}$ together with pSTAT3; this might be triggering inflammatory processes instead of migration in PDAC cells. Nevertheless, since levels of phosphorylated STAT3 were not affected after mutp53 ablation in PANC-1 cells, binding of mutp53 ${ }^{\mathrm{R} 273 \mathrm{H}}$ to pSTAT3 might not result in a direct displacement of phosphatases to regulate STAT3 activity (Manuscript Figure S2B).

To exploit the exact gain-of-function mechanism of the different mutp53 variants, intensive research is required. Binding of mutp53 to pSTAT3 does not necessarily result in a gain-of-function on migration. It is more likely that the exact composition of the complex and the participation of other proteins and transcription factors might define which tumorigenic function is gained. Because of this vast complexity and heterogeneity, it is important to further investigate the underlying mechanism of each p53 mutant in a context-specific manner.

\subsubsection{Stabilized mutp53 as potential therapeutic target}

Since TP53 is mutated in the majority of cancers, resulting in its accumulation especially in cancer cells compared to normal cells [285], several efforts have been made in different cancer entities to specifically target mutp53 in tumor cells e.g. via HSP90 inhibition [56, 85, 226].

To investigate whether mutp53 is also a potential drug target in PDAC, we treated different PDAC cells with the two HSP90 inhibitors Gantespib and Onalespib. Interestingly, HSP90 inhibition results in destabilization of the mutp53 protein in all analyzed PDAC cells except BXPC-3 cells (Manuscript Figure 2A). However, the 
positive control AKT also failed to be degraded upon inhibitor treatment in BXPC-3 cells. These data suggest a resistance mechanism towards HSP90 inhibition. As previously shown in $C R C$, that resistance towards Ganetespib correlates with the expression of UDP glucuronosyltransferase 1A (UGT1A) [286]. UGT1A belongs to a superfamily of proteins involved in the biotransformation and excretion of various compounds and proteins [286, 287]. An overexpression of UGT1A in BXPC-3 cells could provide an explanation for the resistance towards both HSP90 inhibitors.

As in our current PDAC study, many other studies have also shown that different mutp53 proteins are stabilized by the HSP90 chaperone machinery, resulting in elevated mutp53 levels in cancer cells [56, 85, 214, 229, 288]. In addition, Parrales et al. showed that mutp53 can be targeted by interference with the mevalonate pathway, using the small molecule inhibitor Statin [289]. An intermediate of this pathway (mevalonate-5-phosphate) contributes to the inhibition of the ubiquitin ligase CHIP by promoting the interaction between HSP40 and mutp53 [289, 290]. Inhibition of this intermediate results in an increased CHIP-mediated degradation of mutp53 [289, 290]. Interestingly, one of the investigated cell lines in their study is the BXPC-3 cell line harboring the conformational mutant mutp53 ${ }^{\mathrm{Y} 220 \mathrm{C}}[290]$. The fact that mutp53 ${ }^{\mathrm{Y} 220 \mathrm{C}}$ was not targetable using HSP90 inhibitors (Manuscript Figure 2A) could also be explained, by HSP90-independent stabilization via interaction with other cochaperones such as HSP40 [290]. Taken together these results highlight that the entire HSP machinery is essential for stabilization of mutp53 which is required for its gain-offunction activities.

It has been demonstrated that high levels of stabilized mutp53 are a prerequisite for the gain of new tumorigenic functions [221, 222]. However, some controversies exist in the literature concerning the exact mechanism that leads to elevated mutp53 levels. It is important to note that mutation-induced unstable conformations of some mutant p53 proteins such as R248Q, R273H, R175H, R282W and Y220C, might contribute to an aggregation of these mutants, either amongst themselves and/or with other proteins [217, 291, 292], independent of HSP90. Mutations-induced conformational changes of p53 can expose an aggregation-prone sequence within the hydrophopic core of DNA binding domain resulting in oligomerization [218]. These prion-like aggregates were shown to interact and thereby modulate the transcriptional activity of other proteins such as remaining WTp53 as well as p53-family members such as p63 and p73 and 
$[217,219,220,293]$. BXPC-3 cells display the Y220C mutation of $p 53$, which was described to form oligomeric structures [291]. Therefore, further studies are needed to elucidate whether higher concentrations of the HSP90 inhibitors are able to induce degradation of mutp53 in these cells at all. Treatment with Statins to inhibit the mevalonate pathway could provide some evidence whether mutp $53^{\mathrm{Y} 220 \mathrm{C}}$ is stabilized in a HSP40-dependent manner in PDAC [290]. Altogether, this would help to understand if mutp53 ${ }^{\mathrm{Y} 220 \mathrm{C}}$ is a client of HSP90/HSP40 in pancreatic cancer cells or if moderately elevated levels of mutp53 in these cells are due to self-aggregation of the protein. Co-immunoprecipitation of mutp53 ${ }^{\mathrm{Y} 220 \mathrm{C}}$ and HSP90/HSP40 would help to further elucidate whether this mutant in BXPC-3 cells is a client of HSPs or not.

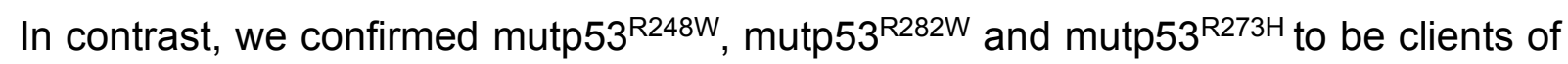
the HSP90 chaperone machinery in PDAC (Manuscript Figure 2A). In fact, cancer cells might even have an equilibrium of HSP90-stabilized and aggregated proteins, resulting in the gain of new tumorigenic functions via interaction of mutp53 with other proteins to form complexes in general [217]. The exact mechanism might be highly dependent on the cellular and molecular context. Of note, small molecules, such as ReACp53 or PRIMA-1, targeting these prion-like heterogeneous aggregates of mutp53 have been applied to preclinical and clinical trials [228, 291, 294]. This approach focuses on personalized drugs in order to change the conformation of different p53 mutants, leading to their disaggregation or the restoration of wildtype p53 functions (reactivators) [228, 291].

Further investigation of the transient and dynamic nature of these complexes is necessary to obtain a better understanding of the different mutp53 GOFs [291]. In the current study we provided first evidence that most of the p53 mutants (except Y220C) can serve as potential biomarkers for HSP90 inhibitor treatment. Given the plethora of HSP90 clients, simultaneous inhibition of cancer-relevant clients can help to overcome possible therapy induced resistance mechanisms by targeting more than one tumor driving mechanisms $[19,235]$.

Apart from the strategies discussed above to target mutp53 itself (HSP90 inhibitors, Statins, p53 reactivators), it would also be possible to target mutp53 through targeting its interaction partners to disrupt hetero TF complexes. Hence, in the context of mutp53 ${ }^{\mathrm{R} 248 \mathrm{~W}}$, it would be of particular interest to target pSTAT3. In this context, our study revealed that siRNA-mediated knockdown or inhibition of STAT3 using Stattic, 
resulted in diminished migration specifically in mutp53 $248 \mathrm{~W}$ harboring MIA-PACA-2 cells (Manuscript Figure 5A, B, D). Additionally, MIA-PACA-2 cells were most susceptible towards Stattic treatment compared to other PDAC cells (Manuscript Figure 5C). However, Stattic was tested in several other preclinical trials, but is not considered a potent therapeutic candidate, due to diverse off-target effects and lacking efficacy in vivo [295-297]. In contrast BBI608 (Napabucasin), a small molecule shown to inhibit stemness pathways such as the STAT3 signaling pathway [298] has achieved orphan status by the FDA for treatment of gastric and pancreatic cancers [279, 299]. Unfortunately, phase III clinical trial for both cancers were discontinued due to futility $[298,300]$. In contrast, mutp53 ${ }^{\mathrm{R} 273 \mathrm{H}}$ might form hetero TF complexes with NF- $\kappa \mathrm{B}$ and other cofactors such as pSTAT3 (Manuscript Figure 4B) causing a GOF on inflammation rather than migration [281]. In that case, a modulator of inflammatory pathways might be more relevant Interestingly, the triterpenoid bardoxolone methyl (RTA 402 or CDDO-Me) was found to inhibit both: STAT3 and NF- $\kappa$ B pathways [301304]. First clinical evaluations for advanced solid tumors showed low toxicity and promising anti-tumor efficacy [303].

Using inhibitors for the specific hetero TF complexes based on specific biomarkers might be an important step towards personalized medicine, helping to increase success rates of pancreatic cancer treatments. A combination of novel HSP90 inhibitors (section 2.1.3) together with mutp53 variant-specific STAT3/NF- $\kappa B$ inhibitors (BBI608, RTA 402) could provide a good treatment option to selectively target tumor cells and prevent acquisition of resistance [19, 235].

\subsubsection{Mutp53 and the PDAC tumor microenvironment}

In the past couple of years, it became more evident that mutp53 can influence the crosstalk between cancer cells and the components of the tumor microenvironment [305]. It is thought that the interaction of mutp53 variants with different transcription factors (e.g., NF- $\kappa \mathrm{B}, \mathrm{pSTAT3}$, see section 4.2.1) can trigger a pro-tumorigenic response of stromal cells either by specific secretion of soluble bioactive mediators (e.g. cytokines, angiogenic factors) or the transcription of proteins which are necessary for interaction between tumor and stromal cells [305-307]. In that way, mutp53 can 
regulate the exact composition of the cancer secretome in order to acquire new tumorigenic functions and to promote tumor progression [305].

PDAC is histologically characterized by a very high stromal content (approx. $90 \%$ of the tumor bulk), also contributing to the broad heterogeneity and plasticity of the disease as well as therapeutic resistance $[178,179]$. Because of that, it is especially important for further studies to take the tumor microenvironment into consideration. A recent publication from Butera et al. investigated the impact of different mutp53 proteins on the tumor secretome [308]. They found that mutp53 ${ }^{\mathrm{R} 175 \mathrm{H}}$ and mutp53 ${ }^{\mathrm{R} 273 \mathrm{H}}$ can alter the cancer secretome to promote anti-apoptotic and hyperproliferative responses as well as chemoresistance of PDAC cells [308]. Interestingly, closer analysis of the exact composition of secreted proteins revealed a vast interaction network with several proteins, including HSP90 [308]. This is in line with our study presenting mutp53 ${ }^{\mathrm{R} 273 \mathrm{H}}$ as a HSP90 client in PDAC (Manuscript Figure 2A). Taken together, HSP90-stabilized mutp53 ${ }^{\mathrm{R} 273 \mathrm{H}}$ in PDAC cells, might lead to the formation of hetero TF complexes with NF- $\kappa \mathrm{B}$, pSTAT3 and potential other cofactors to promote inflammatory processes and to regulate the cancer secretome [281, 308]. Thus, mutp53 ${ }^{\mathrm{R} 273 \mathrm{H}}$ in PANC-1 cells might constitute a GOF on inflammation, apoptosis or chemoresistance instead of migration.

Since PDAC is a cancer entity with high desmoplastic reaction, the interaction of the tumor microenvironment (predominantly cancer-associated fibroblasts, CAFs) and cancer cells has to be taken into account [309]. Models like patient-derived xenograft and 3D organoid co-cultures harboring different p53 mutations could allow a crosstalk between stromal and cancer cells, mimicking the broad tumor heterogeneity in human PDAC patients [309, 310]. In vivo studies and in vitro co-cultures with stromal compartments of the tumor microenvironment can help to clarify the impact of different mutants on the composition of the secretome, which might help to better understand this broad heterogeneity.

In the context of tumor microenvironment, tumor associated macrophages have been extensively studied and are suggested to significantly contribute to tumor progression [170, 311]. Cooks et al. propose that mutp53 in colorectal cancer cells, promotes increased secretion of miR-1246 containing exosomes [311]. Uptake of these exosomes by macrophages results in a phenotypic shift towards M2 
(immunosuppressive, tumor-promoting) contributing to the progression and metastasis of cancer [307, 311].

In sum, the current study investigates the role of different p53 mutants in PDAC, showing that different mutations are differentially stabilized and that only the HSP90stabilized R248W mutant has a GOF on migration. Again, these data further emphasize the necessity to investigate the role of mutp53 in a mutant variant and context-specific manner. Additionally, it highlights that different mutants of p53 can have different GOFs which might be due to different interaction partners and the formation of hetero TF complexes. However, intensive research is needed to fully understand the underlying GOF mechanism and the clinical potential in cancer entities with inadequate therapeutic approaches, such as PDAC.

\subsection{CONCLUSION}

In our studies, we aimed to investigated HSP90-stabilized proteins as therapeutic targets in cancer.

We show that mutp53 ${ }^{R 248 \mathrm{~W}}$ is an important HSP90 client in pancreatic ductal adenocarcinoma driving migration via hijacking of PSTAT3 (Manuscript, section 3.2). Our data provide first evidence that the mutp53 ${ }^{\mathrm{R} 248 \mathrm{~W}}$ variant is a cancer-relevant HSP90 client in PDAC and that its ablation reduces migration of cancer cells. However, further research is necessary to elaborate on the exact mechanism of action. Additional experimental models could be deployed, to better understand the complexity and the impact of stabilized mutp53 variants in cancer cells on the interaction with other compartments of the tumor bulk (e.g., cancer-associated fibroblasts or immune cells).

Furthermore, our data highlight MIF as a cancer-relevant HSP90 client in colorectal cancer which can serve as target for cancer therapy (Publication, section 3.1). These data are in line with different studies suggesting MIF as a potential target in other models for intestinal cancer [111, 149] and other cancer entities [57, 112, 312]. So far, little is known about the exact mechanism leading to the stabilization of MIF through the HSP90 chaperone machinery. The oxidation of MIF might provide an explanation but needs to be further analyzed. Our findings, that MIF is dispensable for normal but not for cancer cells make it an interesting target for cancer therapy. In normal cells, 
other pro-inflammatory cytokines might be able take over the function after MIF depletion. In contrast, tumor cells are addicted to tumorigenic function of HSP90stabilized MIF. Thus, the combination of novel HPS90 inhibitors and anti-MIF or antiCD74 treatments can provide an opportunity to better disrupt cancer cells.

In this respect, it might also be of interest to investigate whether MIF can serve as potential target in PDAC. Two studies have proven that MIF promotes tumor aggressiveness, metastasis and invasion in PDAC [312, 313]. Thus, elevated MIF levels were also a predictor for worse prognosis for PDAC patients [312, 314]. Especially in a stromal dense cancer entity like PDAC [179], investigating the impact of MIF especially on the regulation and activation of macrophages and their contribution to tumor angiogenesis might be of interest. Therapeutically, it would be interesting to understand if MIF can serve as a cancer-relevant HSP90 client also in PDAC.

To date, none of the tested HSP90 inhibitors have passed clinical validation and achieved FDA approval [14, 91]. Nevertheless, the vast plethora of clients and the specific higher affinity of inhibitors to tumoral Hsp90 make it a preferable target for cancer therapies $[21,68,79]$. Simultaneous inhibition of cancer-relevant HSP90 clients can also help to overcome resistance mechanism $[19,235]$. To improve the clinical outcome of HSP90 inhibitors, it is important to investigate possible cancer-relevant HSP90 clients that could help to increase efficacy of these inhibitors. Additionally, a combination treatment of HSP90 inhibitors and compounds against specific biomarkers such as MIF, mutp53 or mutp53-specific hetero TF complexes could be of interest to improve the success of cancer therapy. 


\section{REFERENCES}

1. National Cancer Institute, Cancer Statistics. 2020 [cited 15.12.2020]; Available from: https://www.cancer.gov/about-cancer/understanding/statistics.

2. Sung H, Ferlay J, Siegel RL, Laversanne M, Soerjomataram I, et al., Global cancer statistics 2020: GLOBOCAN estimates of incidence and mortality worldwide for 36 cancers in 185 countries. CA Cancer J Clin, 2021. DOI: $10.3322 /$ caac. 21660

3. The global challenge of cancer. Nature Cancer, 2020. 1, 1-2 DOI: 10.1038/s43018-019-0023-9.

4. Cagan R and Meyer P, Rethinking cancer: current challenges and opportunities in cancer research. Dis Model Mech, 2017. 10(4): p. 349-352. DOI: $10.1242 / \mathrm{dmm} .030007$

5. Wong $\mathrm{CH}$, Siah $\mathrm{KW}$, and Lo AW, Estimation of clinical trial success rates and related parameters. Biostatistics, 2019. 20(2): p. 273-286. DOI: 10.1093/biostatistics/kxx069

6. Dietlein F, Weghorn D, Taylor-Weiner A, Richters A, Reardon B, et al., Identification of cancer driver genes based on nucleotide context. Nat Genet, 2020. 52(2): p. 208-218. DOI: 10.1038/s41588-019-0572-y

7. Hanahan D and Weinberg RA, The hallmarks of cancer. Cell, 2000. 100(1): p. 57-70. DOI: 10.1016/s0092-8674(00)81683-9

8. Greenman C, Stephens P, Smith R, Dalgliesh GL, Hunter C, et al., Patterns of somatic mutation in human cancer genomes. Nature, 2007. 446(7132): p. 1538. DOI: $10.1038 /$ nature 05610

9. Loeb LA, Loeb KR, and Anderson JP, Multiple mutations and cancer. Proc Natl Acad Sci U S A, 2003. 100(3): p. 776-81. DOI: 10.1073/pnas.0334858100

10. Chakravarthi BV, Nepal S, and Varambally S, Genomic and Epigenomic Alterations in Cancer. Am J Pathol, 2016. 186(7): p. 1724-35. DOI: 10.1016/j.ajpath.2016.02.023

11. Senft D and Ronai ZA, Adaptive Stress Responses During Tumor Metastasis and Dormancy. Trends Cancer, 2016. 2(8): p. 429-442. DOI: 10.1016/j.trecan.2016.06.004

12. Xu W, Trepel J, and Neckers L, Ras, ROS and proteotoxic stress: a delicate balance. Cancer Cell, 2011. 20(3): p. 281-2. DOI: 10.1016/j.ccr.2011.08.020

13. Ruggero D, Translational control in cancer etiology. Cold Spring Harb Perspect Biol, 2013. 5(2). DOI: 10.1101/cshperspect.a012336

14. Jaeger AM and Whitesell L, HSP90: Enabler of Cancer Adaptation. Annual Review of Cancer Biology, 2019. 3(1): p. 275-297. DOI: 10.1146/annurevcancerbio-030518-055533

15. Guang MHZ, Kavanagh EL, Dunne LP, Dowling P, Zhang L, et al., Targeting Proteotoxic Stress in Cancer: A Review of the Role that Protein Quality Control 
Pathways Play in Oncogenesis. Cancers (Basel), 2019. 11(1). DOI: 10.3390/cancers 11010066

16. Whitesell $L$ and Lindquist SL, HSP90 and the chaperoning of cancer. Nat Rev Cancer, 2005. 5(10): p. 761-72. DOI: 10.1038/nrc1716

17. Miyata $\mathrm{Y}$, Nakamoto $\mathrm{H}$, and Neckers $\mathrm{L}$, The therapeutic target Hsp90 and cancer hallmarks. Curr Pharm Des, 2013. 19(3): p. 347-65. DOI: 10.2174/138161213804143725

18. Acquaviva J, Smith DL, Jimenez JP, Zhang C, Sequeira M, et al., Overcoming acquired BRAF inhibitor resistance in melanoma via targeted inhibition of Hsp90 with ganetespib. Mol Cancer Ther, 2014. 13(2): p. 353-63. DOI: 10.1158/15357163.MCT-13-0481

19. Jhaveri K and Modi S, HSP90 inhibitors for cancer therapy and overcoming drug resistance. Adv Pharmacol, 2012. 65: p. 471-517. DOI: 10.1016/B978-0-12397927-8.00015-4

20. Jhaveri K and Modi S, Ganetespib: research and clinical development. Onco Targets Ther, 2015. 8: p. 1849-58. DOI: 10.2147/OTT.S65804

21. Picard, D., HSP90 INTERACTORS. 2021 [cited 31.03.2021]; Available from: https://www.picard.ch/downloads/Hsp90interactors.pdf.

22. Lindquist S and Craig EA, The heat-shock proteins. Annu Rev Genet, 1988. 22 : p. 631-77. DOI: 10.1146/annurev.ge.22.120188.003215

23. Taipale M, Jarosz DF, and Lindquist S, HSP9O at the hub of protein homeostasis: emerging mechanistic insights. Nat Rev Mol Cell Biol, 2010. 11(7): p. 515-28. DOI: $10.1038 / \mathrm{nrm} 2918$

24. Csermely P, Schnaider T, Soti C, Prohaszka Z, and Nardai G, The 90-kDa molecular chaperone family: structure, function, and clinical applications. A comprehensive review. Pharmacol Ther, 1998. 79(2): p. 129-68. DOI: 10.1016/s0163-7258(98)00013-8

25. Ritossa $F, A$ new puffing pattern induced by temperature shock and DNP in drosophila. Experientia, 1962. 18(12): p. 571-573. DOI: 10.1007/BF02172188

26. Bakthisaran R, Tangirala R, and Rao Ch M, Small heat shock proteins: Role in cellular functions and pathology. Biochim Biophys Acta, 2015. 1854(4): p. 291319. DOI: 10.1016/j.bbapap.2014.12.019

27. De Maio A, Heat shock proteins: facts, thoughts, and dreams. Shock, 1999. 11(1): p. 1-12. DOI: 10.1097/00024382-199901000-00001

28. Li Z and Srivastava P, Heat-shock proteins. Curr Protoc Immunol, 2004. Appendix 1: p. Appendix 1T. DOI: 10.1002/0471142735.ima01ts58

29. Li J, Richter K, and Buchner J, Mixed Hsp90-cochaperone complexes are important for the progression of the reaction cycle. Nat Struct Mol Biol, 2011. 18(1): p. 61-6. DOI: 10.1038/nsmb.1965

30. Geller R, Taguwa S, and Frydman J, Broad action of Hsp90 as a host chaperone required for viral replication. Biochim Biophys Acta, 2012. 1823(3): p. 698-706. DOI: 10.1016/j.bbamcr.2011.11.007

31. Picard D, Heat-shock protein 90, a chaperone for folding and regulation. Cell Mol Life Sci, 2002. 59(10): p. 1640-8. DOI: 10.1007/pl00012491 
32. Lai BT, Chin NW, Stanek AE, Keh W, and Lanks KW, Quantitation and intracellular localization of the $85 \mathrm{~K}$ heat shock protein by using monoclonal and polyclonal antibodies. Mol Cell Biol, 1984. 4(12): p. 2802-10. DOI: 10.1128/mcb.4.12.2802

33. Sreedhar AS, Kalmar E, Csermely P, and Shen YF, Hsp90 isoforms: functions, expression and clinical importance. FEBS Lett, 2004. 562(1-3): p. 11-5. DOI: 10.1016/s0014-5793(04)00229-7

34. Perdew GH, Hord N, Hollenback CE, and Welsh MJ, Localization and characterization of the 86- and 84-kDa heat shock proteins in Hepa 1c1c7 cells. Exp Cell Res, 1993. 209(2): p. 350-6. DOI: 10.1006/excr.1993.1320

35. Richter K, Muschler P, Hainzl O, and Buchner J, Coordinated ATP hydrolysis by the Hsp90 dimer. J Biol Chem, 2001. 276(36): p. 33689-96. DOI: 10.1074/jbc.M103832200

36. Wayne $\mathrm{N}$ and Bolon DN, Dimerization of Hsp90 is required for in vivo function. Design and analysis of monomers and dimers. J Biol Chem, 2007. 282(48): p. 35386-95. DOI: 10.1074/jbc.M703844200

37. Nemoto T, Ohara-Nemoto Y, Ota M, Takagi T, and Yokoyama K, Mechanism of dimer formation of the 90-kDa heat-shock protein. Eur J Biochem, 1995. 233(1): p. 1-8. DOI: 10.1111/j.1432-1033.1995.001_1.x

38. Schopf FH, Biebl MM, and Buchner J, The HSP9O chaperone machinery. Nat Rev Mol Cell Biol, 2017. 18(6): p. 345-360. DOI: 10.1038/nrm.2017.20

39. Li J, Soroka J, and Buchner J, The Hsp90 chaperone machinery: conformational dynamics and regulation by co-chaperones. Biochim Biophys Acta, 2012. 1823(3): p. 624-35. DOI: 10.1016/j.bbamcr.2011.09.003

40. Hernandez MP, Chadli A, and Toft DO, HSP40 binding is the first step in the HSP90 chaperoning pathway for the progesterone receptor. J Biol Chem, 2002. 277(14): p. 11873-81. DOI: 10.1074/jbc.M111445200

41. Johnson BD, Schumacher RJ, Ross ED, and Toft DO, Hop modulates Hsp70/Hsp90 interactions in protein folding. J Biol Chem, 1998. 273(6): p. 367986. DOI: $10.1074 / \mathrm{jbc} .273 .6 .3679$

42. Hoter A, El-Sabban ME, and Naim HY, The HSP9O Family: Structure, Regulation, Function, and Implications in Health and Disease. Int J Mol Sci, 2018. 19(9). DOI: 10.3390/ijms19092560

43. Wang H, Tan MS, Lu RC, Yu JT, and Tan L, Heat shock proteins at the crossroads between cancer and Alzheimer's disease. Biomed Res Int, 2014. 2014: p. 239164. DOI: $10.1155 / 2014 / 239164$

44. Lanneau D, Wettstein G, Bonniaud P, and Garrido C, Heat shock proteins: cell protection through protein triage. ScientificWorldJournal, 2010. 10: p. 1543-52. DOI: 10.1100/tsw.2010.152

45. Zuehlke $\mathrm{A}$ and Johnson $\mathrm{JL}, \mathrm{Hsp} 90$ and co-chaperones twist the functions of diverse client proteins. Biopolymers, 2010. 93(3): p. 211-7. DOI: 10.1002/bip.21292

46. Scroggins BT, Robzyk K, Wang D, Marcu MG, Tsutsumi S, et al., An acetylation site in the middle domain of Hsp90 regulates chaperone function. Mol Cell, 2007. 25(1): p. 151-9. DOI: 10.1016/j.molcel.2006.12.008 
47. Riggs DL, Cox MB, Cheung-Flynn J, Prapapanich V, Carrigan PE, et al., Functional specificity of co-chaperone interactions with Hsp90 client proteins. Crit Rev Biochem Mol Biol, 2004. 39(5-6): p. 279-95. DOI: 10.1080/10409230490892513

48. Sahasrabudhe P, Rohrberg J, Biebl MM, Rutz DA, and Buchner J, The Plasticity of the Hsp90 Co-chaperone System. Mol Cell, 2017. 67(6): p. 947-961 e5. DOI: 10.1016/j.molcel.2017.08.004

49. Akerfelt M, Morimoto RI, and Sistonen L, Heat shock factors: integrators of cell stress, development and lifespan. Nat Rev Mol Cell Biol, 2010. 11(8): p. 54555. DOI: $10.1038 / \mathrm{nrm} 2938$

50. Prodromou C, Mechanisms of Hsp90 regulation. Biochem J, 2016. 473(16): p. 2439-52. DOI: 10.1042/BCJ20160005

51. Vabulas RM, Raychaudhuri S, Hayer-Hartl M, and Hartl FU, Protein folding in the cytoplasm and the heat shock response. Cold Spring Harb Perspect Biol, 2010. 2(12): p. a004390. DOI: $10.1101 /$ cshperspect.a004390

52. Neef DW, Jaeger AM, Gomez-Pastor R, Willmund F, Frydman J, et al., A direct regulatory interaction between chaperonin TRiC and stress-responsive transcription factor HSF1. Cell Rep, 2014. 9(3): p. 955-66. DOI: 10.1016/j.celrep.2014.09.056

53. Zou J, Guo Y, Guettouche T, Smith DF, and Voellmy R, Repression of heat shock transcription factor HSF1 activation by HSP90 (HSP90 complex) that forms a stress-sensitive complex with HSF1. Cell, 1998. 94(4): p. 471-80. DOI: 10.1016/s0092-8674(00)81588-3

54. Gomez-Pastor R, Burchfiel ET, and Thiele DJ, Regulation of heat shock transcription factors and their roles in physiology and disease. Nat Rev Mol Cell Biol, 2018. 19(1): p. 4-19. DOI: 10.1038/nrm.2017.73

55. Luo W, Sun W, Taldone T, Rodina A, and Chiosis G, Heat shock protein 90 in neurodegenerative diseases. Mol Neurodegener, 2010. 5: p. 24. DOI: 10.1186/1750-1326-5-24

56. Schulz-Heddergott R, Stark N, Edmunds SJ, Li J, Conradi LC, et al., Therapeutic Ablation of Gain-of-Function Mutant p53 in Colorectal Cancer Inhibits Stat3-Mediated Tumor Growth and Invasion. Cancer Cell, 2018. 34(2): p. 298-314 e7. DOI: 10.1016/j.ccell.2018.07.004

57. Schulz R, Marchenko ND, Holembowski L, Fingerle-Rowson G, Pesic M, et al., Inhibiting the HSP90 chaperone destabilizes macrophage migration inhibitory factor and thereby inhibits breast tumor progression. J Exp Med, 2012. 209(2): p. 275-89. DOI: 10.1084/jem.20111117

58. McDowell CL, Bryan Sutton R, and Obermann WM, Expression of Hsp90 chaperone [corrected] proteins in human tumor tissue. Int J Biol Macromol, 2009. 45(3): p. 310-4. DOI: 10.1016/j.ijbiomac.2009.06.012

59. Pick E, Kluger Y, Giltnane JM, Moeder C, Camp RL, et al., High HSP90 expression is associated with decreased survival in breast cancer. Cancer Res, 2007. 67(7): p. 2932-7. DOI: 10.1158/0008-5472.CAN-06-4511 
60. Yano M, Naito Z, Tanaka S, and Asano G, Expression and roles of heat shock proteins in human breast cancer. Jpn J Cancer Res, 1996. 87(9): p. 908-15. DOI: $10.1111 / \mathrm{j} .1349-7006.1996 . t b 02119 . x$

61. Zhang S, Guo S, Li Z, Li D, and Zhan Q, High expression of HSP9O is associated with poor prognosis in patients with colorectal cancer. PeerJ, 2019. 7: p. e7946. DOI: 10.7717/peerj.7946

62. Kim K, Lee HW, Lee EH, Park MI, Lee JS, et al., Differential expression of HSP90 isoforms and their correlations with clinicopathologic factors in patients with colorectal cancer. Int J Clin Exp Pathol, 2019. 12(3): p. 978-986.

63. Gress TM, Muller-Pillasch F, Weber C, Lerch MM, Friess H, et al., Differential expression of heat shock proteins in pancreatic carcinoma. Cancer Res, 1994. 54(2): p. 547-51.

64. Ogata M, Naito Z, Tanaka S, Moriyama Y, and Asano G, Overexpression and localization of heat shock proteins $m R N A$ in pancreatic carcinoma. J Nippon Med Sch, 2000. 67(3): p. 177-85. DOI: 10.1272/jnms.67.177

65. Walczak A, Gradzik K, Kabzinski J, Przybylowska-Sygut K, and Majsterek I, The Role of the ER-Induced UPR Pathway and the Efficacy of Its Inhibitors and Inducers in the Inhibition of Tumor Progression. Oxid Med Cell Longev, 2019. 2019: p. 5729710 . DOI: $10.1155 / 2019 / 5729710$

66. Takayama S, Reed JC, and Homma S, Heat-shock proteins as regulators of apoptosis. Oncogene, 2003. 22(56): p. 9041-7. DOI: 10.1038/sj.onc.1207114

67. Seo YH, Small Molecule Inhibitors to Disrupt Protein-protein Interactions of Heat Shock Protein 90 Chaperone Machinery. J Cancer Prev, 2015. 20(1): p. 5-11. DOI: 10.15430/JCP.2015.20.1.5

68. Workman $\mathrm{P}$, Altered states: selectively drugging the Hsp90 cancer chaperone. Trends Mol Med, 2004. 10(2): p. 47-51. DOI: 10.1016/j.molmed.2003.12.005

69. Kamal A, Boehm MF, and Burrows FJ, Therapeutic and diagnostic implications of Hsp90 activation. Trends Mol Med, 2004. 10(6): p. 283-90. DOI: 10.1016/j.molmed.2004.04.006

70. Dai C, The heat-shock, or HSF1-mediated proteotoxic stress, response in cancer: from proteomic stability to oncogenesis. Philos Trans R Soc Lond B Biol Sci, 2018. 373(1738). DOI: 10.1098/rstb.2016.0525

71. Kamal A, Thao L, Sensintaffar J, Zhang L, Boehm MF, et al., A high-affinity conformation of Hsp90 confers tumour selectivity on Hsp90 inhibitors. Nature, 2003. 425(6956): p. 407-10. DOI: 10.1038/nature01913

72. Mahalingam D, Swords R, Carew JS, Nawrocki ST, Bhalla K, et al., Targeting HSP90 for cancer therapy. Br J Cancer, 2009. 100(10): p. 1523-9. DOI: 10.1038/sj.bjc.6605066

73. Hanahan D and Weinberg RA, Hallmarks of cancer: the next generation. Cell, 2011. 144(5): p. 646-74. DOI: 10.1016/j.cell.2011.02.013

74. Vahid S, Thaper D, and Zoubeidi A, Chaperoning the Cancer: The Proteostatic Functions of the Heat Shock Proteins in Cancer. Recent Pat Anticancer Drug Discov, 2017. 12(1): p. 35-47. DOI: 10.2174/1574892811666161102125252 
75. Trepel J, Mollapour M, Giaccone G, and Neckers L, Targeting the dynamic HSP90 complex in cancer. Nat Rev Cancer, 2010. 10(8): p. 537-49. DOI: $10.1038 / \mathrm{nrc} 2887$

76. Schulz R, Streller F, Scheel AH, Ruschoff J, Reinert MC, et al., HER2/ErbB2 activates HSF1 and thereby controls HSP90 clients including MIF in HER2overexpressing breast cancer. Cell Death Dis, 2014. 5: p. e980. DOI: 10.1038/cddis.2013.508

77. Mimnaugh EG, Chavany C, and Neckers L, Polyubiquitination and proteasomal degradation of the p185c-erbB-2 receptor protein-tyrosine kinase induced by geldanamycin. J Biol Chem, 1996. 271(37): p. 22796-801. DOI: 10.1074/jbc.271.37.22796

78. He S, Zhang C, Shafi AA, Sequeira M, Acquaviva J, et al., Potent activity of the Hsp90 inhibitor ganetespib in prostate cancer cells irrespective of androgen receptor status or variant receptor expression. Int J Oncol, 2013. 42(1): p. 3543. DOI: $10.3892 /$ ijo.2012.1698

79. Neckers L and Lee YS, Cancer: the rules of attraction. Nature, 2003. 425(6956): p. 357-9. DOI: 10.1038/425357a

80. Schulz R and Moll UM, Targeting the heat shock protein 90: a rational way to inhibit macrophage migration inhibitory factor function in cancer. Curr Opin Oncol, 2014. 26(1): p. 108-13. DOI: 10.1097/CCO.0000000000000036

81. Kim YS, Alarcon SV, Lee S, Lee MJ, Giaccone G, et al., Update on Hsp90 inhibitors in clinical trial. Curr Top Med Chem, 2009. 9(15): p. 1479-92. DOI: $10.2174 / 156802609789895728$

82. Jhaveri K, Ochiana SO, Dunphy MP, Gerecitano JF, Corben AD, et al., Heat shock protein 90 inhibitors in the treatment of cancer: current status and future directions. Expert Opin Investig Drugs, 2014. 23(5): p. 611-28. DOI: 10.1517/13543784.2014.902442

83. Parimi S and Tsang RY, Hsp90 inhibitors in oncology: ready for prime time? Curr Oncol, 2014. 21(5): p. e663-7. DOI: 10.3747/co.21.2163

84. Do K, Speranza G, Chang LC, Polley EC, Bishop R, et al., Phase I study of the heat shock protein 90 (Hsp90) inhibitor onalespib (AT13387) administered on a daily for 2 consecutive days per week dosing schedule in patients with advanced solid tumors. Invest New Drugs, 2015. 33(4): p. 921-30. DOI: 10.1007/s10637-015-0255-1

85. Alexandrova EM, Yallowitz AR, Li D, Xu S, Schulz R, et al., Improving survival by exploiting tumour dependence on stabilized mutant p53 for treatment. Nature, 2015. 523(7560): p. 352-6. DOI: 10.1038/nature14430

86. Shimamura T, Perera SA, Foley KP, Sang J, Rodig SJ, et al., Ganetespib (STA9090), a nongeldanamycin HSP90 inhibitor, has potent antitumor activity in in vitro and in vivo models of non-small cell lung cancer. Clin Cancer Res, 2012. 18(18): p. 4973-85. DOI: 10.1158/1078-0432.CCR-11-2967

87. Graham B, Curry J, Smyth T, Fazal L, Feltell R, et al., The heat shock protein 90 inhibitor, AT13387, displays a long duration of action in vitro and in vivo in non-small cell lung cancer. Cancer Sci, 2012. 103(3): p. 522-7. DOI: 10.1111/j.1349-7006.2011.02191.x 
88. Lee H, Saini N, Howard EW, Parris AB, Ma Z, et al., Ganetespib targets multiple levels of the receptor tyrosine kinase signaling cascade and preferentially inhibits ErbB2-overexpressing breast cancer cells. Sci Rep, 2018. 8(1): p. 6829. DOI: $10.1038 / \mathrm{s} 41598-018-25284-0$

89. Kryeziu K, Bruun J, Guren TK, Sveen A, and Lothe RA, Combination therapies with HSP90 inhibitors against colorectal cancer. Biochim Biophys Acta Rev Cancer, 2019. 1871(2): p. 240-247. DOI: 10.1016/j.bbcan.2019.01.002

90. He S, Smith DL, Sequeira M, Sang J, Bates RC, et al., The HSP90 inhibitor ganetespib has chemosensitizer and radiosensitizer activity in colorectal cancer. Invest New Drugs, 2014. 32(4): p. 577-86. DOI: 10.1007/s10637-0140095-4

91. Park HK, Yoon NG, Lee JE, Hu S, Yoon S, et al., Unleashing the full potential of Hsp90 inhibitors as cancer therapeutics through simultaneous inactivation of Hsp90, Grp94, and TRAP1. Exp Mol Med, 2020. 52(1): p. 79-91. DOI: 10.1038/s12276-019-0360-x

92. Eskew JD, Sadikot T, Morales P, Duren A, Dunwiddie I, et al., Development and characterization of a novel C-terminal inhibitor of Hsp90 in androgen dependent and independent prostate cancer cells. BMC Cancer, 2011. 11: p. 468. DOI: 10.1186/1471-2407-11-468

93. Butler LM, Ferraldeschi R, Armstrong HK, Centenera MM, and Workman P, Maximizing the Therapeutic Potential of HSP90 Inhibitors. Mol Cancer Res, 2015. 13(11): p. 1445-51. DOI: 10.1158/1541-7786.MCR-15-0234

94. Neckers L, Blagg B, Haystead T, Trepel JB, Whitesell L, et al., Methods to validate Hsp90 inhibitor specificity, to identify off-target effects, and to rethink approaches for further clinical development. Cell Stress Chaperones, 2018. 23(4): p. 467-482. DOI: 10.1007/s12192-018-0877-2

95. Bickel D and Gohlke H, C-terminal modulators of heat shock protein of $90 \mathrm{kDa}$ (HSP90): State of development and modes of action. Bioorg Med Chem, 2019. 27(21): p. 115080. DOI: 10.1016/j.bmc.2019.115080

96. Marmol I, Sanchez-de-Diego C, Pradilla Dieste A, Cerrada E, and Rodriguez Yoldi MJ, Colorectal Carcinoma: A General Overview and Future Perspectives in Colorectal Cancer. Int J Mol Sci, 2017. 18(1). DOI: 10.3390/ijms18010197

97. Ferlay J, Colombet M, Soerjomataram I, Mathers C, Parkin DM, et al., Estimating the global cancer incidence and mortality in 2018: GLOBOCAN sources and methods. Int J Cancer, 2019. 144(8): p. 1941-1953. DOI: 10.1002/ijc.31937

98. Bray F, Ferlay J, Soerjomataram I, Siegel RL, Torre LA, et al., Global cancer statistics 2018: GLOBOCAN estimates of incidence and mortality worldwide for 36 cancers in 185 countries. CA Cancer J Clin, 2018. 68(6): p. 394-424. DOI: 10.3322/caac.21492

99. Bosman FT, Chapter 5.5: Colorectal Cancer, in World Cancer Report 2014, B.W.W.C.P. Steward, Editor. 2014, International Agency for Research on Cancer, World Health Organization. p. 392 ff. ISBN: 978-92-832-0443-5

100. Vogelstein B, Fearon ER, Hamilton SR, Kern SE, Preisinger AC, et al., Genetic alterations during colorectal-tumor development. N Engl J Med, 1988. 319(9): p. 525-32. DOI: 10.1056/NEJM198809013190901 
101. Fearon ER and Vogelstein B, A genetic model for colorectal tumorigenesis. Cell, 1990. 61(5): p. 759-67. DOI: 10.1016/0092-8674(90)90186-i

102. Xie $\mathrm{J}$ and Itzkowitz $\mathrm{SH}$, Cancer in inflammatory bowel disease. World $\mathrm{J}$ Gastroenterol, 2008. 14(3): p. 378-89. DOI: 10.3748/wjg.14.378

103. Tanaka T, Kohno H, Suzuki R, Yamada Y, Sugie S, et al., A novel inflammationrelated mouse colon carcinogenesis model induced by azoxymethane and dextran sodium sulfate. Cancer Sci, 2003. 94(11): p. 965-73. DOI: 10.1111/j.1349-7006.2003.tb01386.x

104. Rosenberg DW, Giardina C, and Tanaka T, Mouse models for the study of colon carcinogenesis. Carcinogenesis, 2009. 30(2): p. 183-96. DOI: 10.1093/carcin/bgn267

105. De Robertis M, Massi E, Poeta ML, Carotti S, Morini S, et al., The AOM/DSS murine model for the study of colon carcinogenesis: From pathways to diagnosis and therapy studies. J Carcinog, 2011. 10: p. 9. DOI: 10.4103/14773163.78279

106. Chassaing B, Aitken JD, Malleshappa M, and Vijay-Kumar M, Dextran sulfate sodium (DSS)-induced colitis in mice. Curr Protoc Immunol, 2014. 104: p. 1525 1-15 25 14. DOI: 10.1002/0471142735.im1525s104

107. Kuipers EJ, Grady WM, Lieberman D, Seufferlein T, Sung JJ, et al., Colorectal cancer. Nat Rev Dis Primers, 2015. 1: p. 15065. DOI: 10.1038/nrdp.2015.65

108. Walter FM, Emery JD, Mendonca S, Hall N, Morris HC, et al., Symptoms and patient factors associated with longer time to diagnosis for colorectal cancer: results from a prospective cohort study. Br J Cancer, 2016. 115(5): p. 533-41. DOI: 10.1038/bjc.2016.221

109. Xu X, Wang B, Ye C, Yao C, Lin Y, et al., Overexpression of macrophage migration inhibitory factor induces angiogenesis in human breast cancer. Cancer Lett, 2008. 261(2): p. 147-57. DOI: 10.1016/j.canlet.2007.11.028

110. Meyer-Siegler K and Hudson PB, Enhanced expression of macrophage migration inhibitory factor in prostatic adenocarcinoma metastases. Urology, 1996. 48(3): p. 448-52. DOI: 10.1016/S0090-4295(96)00207-5

111. He XX, Chen K, Yang J, Li XY, Gan HY, et al., Macrophage migration inhibitory factor promotes colorectal cancer. Mol Med, 2009. 15(1-2): p. 1-10. DOI: 10.2119/molmed.2008.00107

112. Hira E, Ono T, Dhar DK, El-Assal ON, Hishikawa Y, et al., Overexpression of macrophage migration inhibitory factor induces angiogenesis and deteriorates prognosis after radical resection for hepatocellular carcinoma. Cancer, 2005. 103(3): p. 588-98. DOI: $10.1002 /$ cncr.20818

113. Calandra T and Roger T, Macrophage migration inhibitory factor: a regulator of innate immunity. Nat Rev Immunol, 2003. 3(10): p. 791-800. DOI: 10.1038/nri1200

114. Roger T, David J, Glauser MP, and Calandra T, MIF regulates innate immune responses through modulation of Toll-like receptor 4. Nature, 2001. 414(6866): p. 920-4. DOI: 10.1038/414920a 
115. Nishihira J, Macrophage migration inhibitory factor (MIF): its essential role in the immune system and cell growth. J Interferon Cytokine Res, 2000. 20(9): p. 75162. DOI: $10.1089 / 10799900050151012$

116. David JR, Delayed hypersensitivity in vitro: its mediation by cell-free substances formed by lymphoid cell-antigen interaction. Proc Natl Acad Sci U S A, 1966. 56(1): p. 72-7. DOI: 10.1073/pnas.56.1.72

117. Calandra T, Bernhagen J, Mitchell RA, and Bucala R, The macrophage is an important and previously unrecognized source of macrophage migration inhibitory factor. J Exp Med, 1994. 179(6): p. 1895-902. DOI: 10.1084/jem.179.6.1895

118. Bloom BR and Shevach $\mathrm{E}$, Requirement for T cells in the production of migration inhibitory factor. J Exp Med, 1975. 142(5): p. 1306-11. DOI: 10.1084/jem.142.5.1306

119. Suzuki M, Sugimoto H, Nakagawa A, Tanaka I, Nishihira J, et al., Crystal structure of the macrophage migration inhibitory factor from rat liver. Nat Struct Biol, 1996. 3(3): p. 259-66. DOI: 10.1038/nsb0396-259

120. Sun HW, Bernhagen J, Bucala R, and Lolis E, Crystal structure at 2.6-A resolution of human macrophage migration inhibitory factor. Proc Natl Acad Sci U S A, 1996. 93(11): p. 5191-6. DOI: 10.1073/pnas.93.11.5191

121. Rosengren E, Bucala R, Aman P, Jacobsson L, Odh G, et al., The immunoregulatory mediator macrophage migration inhibitory factor (MIF) catalyzes a tautomerization reaction. Mol Med, 1996. 2(1): p. 143-9.

122. Morand EF, Leech M, and Bernhagen J, MIF: a new cytokine link between rheumatoid arthritis and atherosclerosis. Nat Rev Drug Discov, 2006. 5(5): p. 399-410. DOI: 10.1038/nrd2029

123. Asare $\mathrm{Y}$, Schmitt $\mathrm{M}$, and Bernhagen J, The vascular biology of macrophage migration inhibitory factor (MIF). Expression and effects in inflammation, atherogenesis and angiogenesis. Thromb Haemost, 2013. 109(3): p. 391-8. DOI: 10.1160/TH12-11-0831

124. Zernecke A, Bernhagen J, and Weber C, Macrophage migration inhibitory factor in cardiovascular disease. Circulation, 2008. 117(12): p. 1594-602. DOI: 10.1161/CIRCULATIONAHA.107.729125

125. Petralia MC, Battaglia G, Bruno V, Pennisi M, Mangano K, et al., The Role of Macrophage Migration Inhibitory Factor in Alzheimer's Disease: Conventionally Pathogenetic or Unconventionally Protective? Molecules, 2020. 25(2). DOI: 10.3390/molecules25020291

126. Florez-Sampedro L, Soto-Gamez A, Poelarends GJ, and Melgert BN, The role of MIF in chronic lung diseases: looking beyond inflammation. Am J Physiol Lung Cell Mol Physiol, 2020. 318(6): p. L1183-L1197. DOI: 10.1152/ajplung.00521.2019

127. Leng L, Metz CN, Fang Y, Xu J, Donnelly S, et al., MIF signal transduction initiated by binding to CD74. J Exp Med, 2003. 197(11): p. 1467-76. DOI: 10.1084/jem.20030286

128. Bernhagen J, Krohn R, Lue H, Gregory JL, Zernecke A, et al., MIF is a noncognate ligand of $\mathrm{CXC}$ chemokine receptors in inflammatory and 
atherogenic cell recruitment. Nat Med, 2007. 13(5): p. 587-96. DOI: $10.1038 / \mathrm{nm} 1567$

129. Alampour-Rajabi S, El Bounkari O, Rot A, Muller-Newen G, Bachelerie F, et al., MIF interacts with CXCR7 to promote receptor internalization, ERK1/2 and ZAP70 signaling, and lymphocyte chemotaxis. FASEB J, 2015. 29(11): p. 4497-511. DOI: $10.1096 /$ fj.15-273904

130. Jankauskas SS, Wong DWL, Bucala R, Djudjaj S, and Boor P, Evolving complexity of MIF signaling. Cell Signal, 2019. 57: p. 76-88. DOI: 10.1016/j.cellsig.2019.01.006

131. Xie L, Qiao X, Wu Y, and Tang J, beta-Arrestin1 mediates the endocytosis and functions of macrophage migration inhibitory factor. PLoS One, 2011. 6(1): p. e16428. DOI: 10.1371/journal.pone.0016428

132. Schwartz V, Kruttgen A, Weis J, Weber C, Ostendorf T, et al., Role for CD74 and CXCR4 in clathrin-dependent endocytosis of the cytokine MIF. Eur J Cell Biol, 2012. 91(6-7): p. 435-49. DOI: 10.1016/j.ejcb.2011.08.006

133. Tillmann S, Bernhagen $\mathrm{J}$, and Noels $\mathrm{H}$, Arrest Functions of the MIF Ligand/Receptor Axes in Atherogenesis. Front Immunol, 2013. 4: p. 115. DOI: 10.3389/fimmu.2013.00115

134. Shi X, Leng L, Wang T, Wang W, Du X, et al., CD44 is the signaling component of the macrophage migration inhibitory factor-CD74 receptor complex. Immunity, 2006. 25(4): p. 595-606. DOI: 10.1016/j.immuni.2006.08.020

135. Sampey AV, Hall PH, Mitchell RA, Metz CN, and Morand EF, Regulation of synoviocyte phospholipase $A 2$ and cyclooxygenase 2 by macrophage migration inhibitory factor. Arthritis Rheum, 2001. 44(6): p. 1273-80. DOI: 10.1002/15290131(200106)44:6<1273::AID-ART219>3.0.CO;2-8

136. Mitchell RA, Metz CN, Peng $\mathrm{T}$, and Bucala $\mathrm{R}$, Sustained mitogen-activated protein kinase (MAPK) and cytoplasmic phospholipase A2 activation by macrophage migration inhibitory factor (MIF). Regulatory role in cell proliferation and glucocorticoid action. J Biol Chem, 1999. 274(25): p. 18100-6. DOI: 10.1074/jbc.274.25.18100

137. Lang T, Foote A, Lee JP, Morand EF, and Harris J, MIF: Implications in the Pathoetiology of Systemic Lupus Erythematosus. Front Immunol, 2015. 6: p. 577. DOI: 10.3389/fimmu.2015.00577

138. Mitchell RA, Liao $\mathrm{H}$, Chesney J, Fingerle-Rowson $\mathrm{G}$, Baugh J, et al., Macrophage migration inhibitory factor (MIF) sustains macrophage proinflammatory function by inhibiting p53: regulatory role in the innate immune response. Proc Natl Acad Sci U S A, 2002. 99(1): p. 345-50. DOI: 10.1073/pnas.012511599

139. Kleemann R, Hausser A, Geiger G, Mischke R, Burger-Kentischer A, et al., Intracellular action of the cytokine MIF to modulate AP-1 activity and the cell cycle through Jab1. Nature, 2000. 408(6809): p. 211-6. DOI: 10.1038/35041591

140. Bucala R, Signal transduction. A most interesting factor. Nature, 2000. 408(6809): p. 146-7. DOI: 10.1038/35041654 
141. Claret FX, Hibi M, Dhut S, Toda T, and Karin M, A new group of conserved coactivators that increase the specificity of AP-1 transcription factors. Nature, 1996. 383(6599): p. 453-7. DOI: $10.1038 / 383453 a 0$

142. Tomoda K, Kubota Y, and Kato J, Degradation of the cyclin-dependent-kinase inhibitor p27Kip1 is instigated by Jab1. Nature, 1999. 398(6723): p. 160-5. DOI: $10.1038 / 18230$

143. Tomoda K, Yoneda-Kato N, Fukumoto A, Yamanaka S, and Kato JY, Multiple functions of Jab1 are required for early embryonic development and growth potential in mice. J Biol Chem, 2004. 279(41): p. 43013-8. DOI: 10.1074/jbc.M406559200

144. Fan H, Kao W, Yang YH, Gu R, Harris J, et al., Macrophage migration inhibitory factor inhibits the antiinflammatory effects of glucocorticoids via glucocorticoidinduced leucine zipper. Arthritis Rheumatol, 2014. 66(8): p. 2059-70. DOI: 10.1002/art.38689

145. Bucala R and Donnelly SC, Macrophage migration inhibitory factor: a probable link between inflammation and cancer. Immunity, 2007. 26(3): p. 281-5. DOI: 10.1016/j.immuni.2007.03.005

146. Conroy H, Mawhinney L, and Donnelly SC, Inflammation and cancer: macrophage migration inhibitory factor (MIF)--the potential missing link. QJM, 2010. 103(11): p. 831-6. DOI: 10.1093/qjmed/hcq148

147. Meyer-Siegler KL, Bellino MA, and Tannenbaum M, Macrophage migration inhibitory factor evaluation compared with prostate specific antigen as a biomarker in patients with prostate carcinoma. Cancer, 2002. 94(5): p. 1449-56. DOI: 10.1002/cncr.10354

148. Morris KT, Nofchissey RA, Pinchuk IV, and Beswick EJ, Chronic macrophage migration inhibitory factor exposure induces mesenchymal epithelial transition and promotes gastric and colon cancers. PLoS One, 2014. 9(6): p. e98656. DOI: 10.1371/journal.pone.0098656

149. Wilson JM, Coletta PL, Cuthbert RJ, Scott N, MacLennan K, et al., Macrophage migration inhibitory factor promotes intestinal tumorigenesis. Gastroenterology, 2005. 129(5): p. 1485-503. DOI: 10.1053/j.gastro.2005.07.061

150. Sun B, Nishihira J, Suzuki M, Fukushima N, Ishibashi T, et al., Induction of macrophage migration inhibitory factor by lysophosphatidic acid: relevance to tumor growth and angiogenesis. Int J Mol Med, 2003. 12(4): p. 633-41.

151. Ogawa H, Nishihira J, Sato Y, Kondo M, Takahashi N, et al., An antibody for macrophage migration inhibitory factor suppresses tumour growth and inhibits tumour-associated angiogenesis. Cytokine, 2000. 12(4): p. 309-14. DOI: 10.1006/cyto.1999.0562

152. Nalbantoglu I, Blanc V, and Davidson NO, Characterization of Colorectal Cancer Development in Apc (min/+) Mice. Methods Mol Biol, 2016. 1422: p. 309-27. DOI: 10.1007/978-1-4939-3603-8_27

153. Skinner HD, Zheng JZ, Fang J, Agani F, and Jiang BH, Vascular endothelial growth factor transcriptional activation is mediated by hypoxia-inducible factor 1alpha, HDM2, and p70S6K1 in response to phosphatidylinositol 3-kinase/AKT signaling. J Biol Chem, 2004. 279(44): p. 45643-51. DOI: 10.1074/jbc.M404097200 
154. Triner D and Shah YM, Hypoxia-inducible factors: a central link between inflammation and cancer. J Clin Invest, 2016. 126(10): p. 3689-3698. DOI: $10.1172 / \mathrm{JCl} 84430$

155. Shachar I, Cohen S, Marom A, and Becker-Herman S, Regulation of CLL survival by hypoxia-inducible factor and its target genes. FEBS Lett, 2012. 586(18): p. 2906-10. DOI: 10.1016/j.febslet.2012.07.016

156. Zhu G, Tang Y, Geng N, Zheng M, Jiang J, et al., HIF-alpha/MIF and NFkappaB/IL-6 axes contribute to the recruitment of CD11b+Gr-1+ myeloid cells in hypoxic microenvironment of HNSCC. Neoplasia, 2014. 16(2): p. 168-79. DOI: $10.1593 /$ neo.132034

157. Bozzi F, Mogavero A, Varinelli L, Belfiore A, Manenti G, et al., MIF/CD74 axis is a target for novel therapies in colon carcinomatosis. J Exp Clin Cancer Res, 2017. 36(1): p. 16. DOI: 10.1186/s13046-016-0475-z

158. Lee CY, Su MJ, Huang CY, Chen MY, Hsu HC, et al., Macrophage migration inhibitory factor increases cell motility and up-regulates alphavbeta3 integrin in human chondrosarcoma cells. J Cell Biochem, 2012. 113(5): p. 1590-8. DOI: 10.1002/jcb.24027

159. O'Reilly C, Doroudian M, Mawhinney L, and Donnelly SC, Targeting MIF in Cancer: Therapeutic Strategies, Current Developments, and Future Opportunities. Med Res Rev, 2016. 36(3): p. 440-60. DOI: 10.1002/med.21385

160. Lue H, Thiele M, Franz J, Dahl E, Speckgens S, et al., Macrophage migration inhibitory factor (MIF) promotes cell survival by activation of the Akt pathway and role for CSN5/JAB1 in the control of autocrine MIF activity. Oncogene, 2007. 26(35): p. 5046-59. DOI: 10.1038/sj.onc.1210318

161. Noe JT and Mitchell RA, MIF-Dependent Control of Tumor Immunity. Front Immunol, 2020. 11: p. 609948. DOI: 10.3389/fimmu.2020.609948

162. Gordon-Weeks AN, Lim SY, Yuzhalin AE, Jones K, and Muschel R, Macrophage migration inhibitory factor: a key cytokine and therapeutic target in colon cancer. Cytokine Growth Factor Rev, 2015. 26(4): p. 451-61. DOI: 10.1016/j.cytogfr.2015.03.002

163. Sang N, Stiehl DP, Bohensky J, Leshchinsky I, Srinivas V, et al., MAPK signaling up-regulates the activity of hypoxia-inducible factors by its effects on p300. J Biol Chem, 2003. 278(16): p. 14013-9. DOI: 10.1074/jbc.M209702200

164. Oda S, Oda T, Nishi K, Takabuchi S, Wakamatsu T, et al., Macrophage migration inhibitory factor activates hypoxia-inducible factor in a p53-dependent manner. PLoS One, 2008. 3(5): p. e2215. DOI: 10.1371/journal.pone.0002215

165. Swamy MV, Herzog CR, and Rao CV, Inhibition of COX-2 in colon cancer cell lines by celecoxib increases the nuclear localization of active p53. Cancer Res, 2003. 63(17): p. 5239-42.

166. Choi EM, Kim SR, Lee EJ, and Han JA, Cyclooxygenase-2 functionally inactivates $p 53$ through a physical interaction with p53. Biochim Biophys Acta, 2009. 1793(8): p. 1354-65. DOI: 10.1016/j.bbamcr.2009.05.006

167. Yaddanapudi K, Putty K, Rendon BE, Lamont GJ, Faughn JD, et al., Control of tumor-associated macrophage alternative activation by macrophage migration 
inhibitory factor. J Immunol, 2013. 190(6): p. 2984-93. DOI: 10.4049/jimmunol.1201650

168. Mitchell RA and Yaddanapudi K, Stromal-dependent tumor promotion by MIF family members. Cell Signal, 2014. 26(12): p. 2969-78. DOI: 10.1016/j.cellsig.2014.09.012

169. Owen $\mathrm{JL}$ and Mohamadzadeh M, Macrophages and chemokines as mediators of angiogenesis. Front Physiol, 2013. 4: p. 159. DOI: 10.3389/fphys.2013.00159

170. Erreni M, Mantovani A, and Allavena P, Tumor-associated Macrophages (TAM) and Inflammation in Colorectal Cancer. Cancer Microenviron, 2011. 4(2): p. 141-54. DOI: 10.1007/s12307-010-0052-5

171. Russo R, Matrone N, Belli V, Ciardiello D, Valletta M, et al., Macrophage Migration Inhibitory Factor Is a Molecular Determinant of the Anti-EGFR Monoclonal Antibody Cetuximab Resistance in Human Colorectal Cancer Cells. Cancers (Basel), 2019. 11(10). DOI: 10.3390/cancers 11101430

172. Cheon SK, Kim HP, Park YL, Jang JE, Lim Y, et al., Macrophage migration inhibitory factor promotes resistance to MEK blockade in KRAS mutant colorectal cancer cells. Mol Oncol, 2018. 12(8): p. 1398-1409. DOI: 10.1002/1878-0261.12345

173. Fingerle-Rowson G, Kaleswarapu DR, Schlander C, Kabgani N, Brocks T, et al., A tautomerase-null macrophage migration-inhibitory factor (MIF) gene knock-in mouse model reveals that protein interactions and not enzymatic activity mediate MIF-dependent growth regulation. Mol Cell Biol, 2009. 29(7): p. 1922-32. DOI: 10.1128/MCB.01907-08

174. Kok T, Wasiel AA, Cool RH, Melgert BN, Poelarends GJ, et al., Small-molecule inhibitors of macrophage migration inhibitory factor (MIF) as an emerging class of therapeutics for immune disorders. Drug Discov Today, 2018. 23(11): p. 1910-1918. DOI: 10.1016/j.drudis.2018.06.017

175. Cavalli E, Ciurleo R, Petralia MC, Fagone P, Bella R, et al., Emerging Role of the Macrophage Migration Inhibitory Factor Family of Cytokines in Neuroblastoma. Pathogenic Effectors and Novel Therapeutic Targets? Molecules, 2020. 25(5). DOI: 10.3390/molecules25051194

176. Grasso C, Jansen G, and Giovannetti E, Drug resistance in pancreatic cancer: Impact of altered energy metabolism. Crit Rev Oncol Hematol, 2017. 114: p. 139-152. DOI: 10.1016/j.critrevonc.2017.03.026

177. Orth M, Metzger P, Gerum S, Mayerle J, Schneider G, et al., Pancreatic ductal adenocarcinoma: biological hallmarks, current status, and future perspectives of combined modality treatment approaches. Radiat Oncol, 2019. 14(1): p. 141. DOI: 10.1186/s13014-019-1345-6

178. Smigiel JM, Parameswaran N, and Jackson MW, Targeting Pancreatic Cancer Cell Plasticity: The Latest in Therapeutics. Cancers (Basel), 2018. 10(1). DOI: 10.3390/cancers10010014

179. Hessmann E, Buchholz SM, Demir IE, Singh SK, Gress TM, et al., Microenvironmental Determinants of Pancreatic Cancer. Physiol Rev, 2020. 100(4): p. 1707-1751. DOI: 10.1152/physrev.00042.2019 
180. Collisson EA, Sadanandam A, Olson P, Gibb WJ, Truitt M, et al., Subtypes of pancreatic ductal adenocarcinoma and their differing responses to therapy. Nat Med, 2011. 17(4): p. 500-3. DOI: 10.1038/nm.2344

181. Bailey P, Chang DK, Nones K, Johns AL, Patch AM, et al., Genomic analyses identify molecular subtypes of pancreatic cancer. Nature, 2016. 531(7592): p. 47-52. DOI: 10.1038/nature16965

182. Moffitt RA, Marayati R, Flate EL, Volmar KE, Loeza SG, et al., Virtual microdissection identifies distinct tumor- and stroma-specific subtypes of pancreatic ductal adenocarcinoma. Nat Genet, 2015. 47(10): p. 1168-78. DOI: 10.1038/ng.3398

183. Martens S, Lefesvre P, Nicolle R, Biankin AV, Puleo F, et al., Different shades of pancreatic ductal adenocarcinoma, different paths towards precision therapeutic applications. Ann Oncol, 2019. 30(9): p. 1428-1436. DOI: 10.1093/annonc/mdz181

184. Morris JPt, Wang SC, and Hebrok M, KRAS, Hedgehog, Wnt and the twisted developmental biology of pancreatic ductal adenocarcinoma. Nat Rev Cancer, 2010. 10(10): p. 683-95. DOI: 10.1038/nrc2899

185. Heßmann E, The Molecular Frame of Pancreatic Carcinogenesis. 2014: IntechOpen.

186. Guo J, Xie K, and Zheng S, Molecular Biomarkers of Pancreatic Intraepithelial Neoplasia and Their Implications in Early Diagnosis and Therapeutic Intervention of Pancreatic Cancer. Int J Biol Sci, 2016. 12(3): p. 292-301. DOI: 10.7150/ijbs. 14995

187. Cicenas J, Kvederaviciute K, Meskinyte I, Meskinyte-Kausiliene E, Skeberdyte A, et al., KRAS, TP53, CDKN2A, SMAD4, BRCA1, and BRCA2 Mutations in Pancreatic Cancer. Cancers (Basel), 2017. 9(5). DOI: 10.3390/cancers 9050042

188. Lane DP, Cancer. p53, guardian of the genome. Nature, 1992. 358(6381): p. 15-6. DOI: $10.1038 / 358015 \mathrm{a} 0$

189. Lane DP and Crawford LV, $T$ antigen is bound to a host protein in SV40transformed cells. Nature, 1979. 278(5701): p. 261-3. DOI: 10.1038/278261a0

190. Zilfou JT and Lowe SW, Tumor suppressive functions of p53. Cold Spring Harb Perspect Biol, 2009. 1(5): p. a001883. DOI: 10.1101/cshperspect.a001883

191. Moll UM and Slade N, p63 and p73: roles in development and tumor formation. Mol Cancer Res, 2004. 2(7): p. 371-86.

192. Lane DP and Benchimol S, p53: oncogene or anti-oncogene? Genes Dev, 1990. 4(1): p. 1-8. DOI: 10.1101/gad.4.1.1

193. Vousden KH and Lane DP, p53 in health and disease. Nat Rev Mol Cell Biol, 2007. 8(4): p. 275-83. DOI: 10.1038/nrm2147

194. Reed SM and Quelle DE, p53 Acetylation: Regulation and Consequences. Cancers (Basel), 2014. 7(1): p. 30-69. DOI: 10.3390/cancers7010030

195. Moll UM and Petrenko O, The MDM2-p53 interaction. Mol Cancer Res, 2003. 1(14): p. 1001-8.

196. Momand J, Zambetti GP, Olson DC, George D, and Levine AJ, The mdm-2 oncogene product forms a complex with the p53 protein and inhibits $p 53-$ 
mediated transactivation. Cell, 1992. 69(7): p. 1237-45. DOI: 10.1016/00928674(92)90644-r

197. Kamada R, Toguchi Y, Nomura T, Imagawa T, and Sakaguchi K, Tetramer formation of tumor suppressor protein p53: Structure, function, and applications. Biopolymers, 2016. 106(4): p. 598-612. DOI: 10.1002/bip.22772

198. Wu L and Levine AJ, Differential regulation of the p21/WAF-1 and mdm2 genes after high-dose UV irradiation: p53-dependent and p53-independent regulation of the mdm2 gene. Mol Med, 1997. 3(7): p. 441-51.

199. Vousden $\mathrm{KH}$ and Lu X, Live or let die: the cell's response to p53. Nat Rev Cancer, 2002. 2(8): p. 594-604. DOI: $10.1038 / n r c 864$

200. Hollstein M, Sidransky D, Vogelstein B, and Harris CC, p53 mutations in human cancers. Science, 1991. 253(5015): p. 49-53. DOI: 10.1126/science.1905840

201. Vogelstein B, Lane D, and Levine AJ, Surfing the p53 network. Nature, 2000. 408(6810): p. 307-10. DOI: 10.1038/35042675

202. Sabapathy K and Lane DP, Therapeutic targeting of p53: all mutants are equal, but some mutants are more equal than others. Nat Rev Clin Oncol, 2018. 15(1): p. 13-30. DOI: 10.1038/nrclinonc.2017.151

203. Kandoth C, McLellan MD, Vandin F, Ye K, Niu B, et al., Mutational landscape and significance across 12 major cancer types. Nature, 2013. 502(7471): p. 333-339. DOI: 10.1038/nature12634

204. Freed-Pastor WA and Prives C, Mutant p53: one name, many proteins. Genes Dev, 2012. 26(12): p. 1268-86. DOI: 10.1101/gad.190678.112

205. Brosh R and Rotter $\mathrm{V}$, When mutants gain new powers: news from the mutant p53 field. Nat Rev Cancer, 2009. 9(10): p. 701-13. DOI: 10.1038/nrc2693

206. Hollstein $M$, Hergenhahn $M$, Yang $Q$, Bartsch $H$, Wang ZQ, et al., New approaches to understanding p53 gene tumor mutation spectra. Mutat Res, 1999. 431(2): p. 199-209. DOI: 10.1016/s0027-5107(99)00162-1

207. Bullock AN, Henckel J, and Fersht AR, Quantitative analysis of residual folding and DNA binding in mutant p53 core domain: definition of mutant states for rescue in cancer therapy. Oncogene, 2000. 19(10): p. 1245-56. DOI: 10.1038/sj.onc.1203434

208. Sabapathy K, The Contrived Mutant p53 Oncogene - Beyond Loss of Functions. Front Oncol, 2015. 5: p. 276. DOI: 10.3389/fonc.2015.00276

209. Alexandrova EM, Mirza SA, Xu S, Schulz-Heddergott R, Marchenko ND, et al., p53 loss-of-heterozygosity is a necessary prerequisite for mutant p53 stabilization and gain-of-function in vivo. Cell Death Dis, 2017. 8(3): p. e2661. DOI: $10.1038 /$ cddis.2017.80

210. Muller PA and Vousden $\mathrm{KH}, p 53$ mutations in cancer. Nat Cell Biol, 2013. 15(1): p. 2-8. DOI: $10.1038 / \mathrm{ncb} 2641$

211. Bullock AN, Henckel J, DeDecker BS, Johnson CM, Nikolova PV, et al., Thermodynamic stability of wild-type and mutant p53 core domain. Proc Natl Acad Sci U S A, 1997. 94(26): p. 14338-42. DOI: 10.1073/pnas.94.26.14338 
212. Baugh $\mathrm{EH}, \mathrm{Ke} \mathrm{H}$, Levine AJ, Bonneau RA, and Chan CS, Why are there hotspot mutations in the TP53 gene in human cancers? Cell Death Differ, 2018. 25(1): p. 154-160. DOI: $10.1038 / \mathrm{cdd} .2017 .180$

213. Walerych D, Lisek K, and Del Sal G, Mutant p53: One, No One, and One Hundred Thousand. Front Oncol, 2015. 5: p. 289. DOI: 10.3389/fonc. 2015.00289

214. Li D, Marchenko ND, Schulz R, Fischer V, Velasco-Hernandez T, et al., Functional inactivation of endogenous MDM2 and CHIP by HSP9O causes aberrant stabilization of mutant p53 in human cancer cells. Mol Cancer Res, 2011. 9(5): p. 577-88. DOI: 10.1158/1541-7786.MCR-10-0534

215. Blagosklonny MV, Toretsky J, Bohen S, and Neckers L, Mutant conformation of p53 translated in vitro or in vivo requires functional HSP90. Proc Natl Acad Sci U S A, 1996. 93(16): p. 8379-83. DOI: 10.1073/pnas.93.16.8379

216. Ano Bom AP, Rangel LP, Costa DC, de Oliveira GA, Sanches D, et al., Mutant p53 aggregates into prion-like amyloid oligomers and fibrils: implications for cancer. J Biol Chem, 2012. 287(33): p. 28152-62. DOI: 10.1074/jbc.M112.340638

217. Wawrzynow B, Zylicz A, and Zylicz M, Chaperoning the guardian of the genome. The two-faced role of molecular chaperones in p53 tumor suppressor action. Biochim Biophys Acta Rev Cancer, 2018. 1869(2): p. 161-174. DOI: 10.1016/j.bbcan.2017.12.004

218. Xu J, Reumers J, Couceiro JR, De Smet F, Gallardo R, et al., Gain of function of mutant $p 53$ by coaggregation with multiple tumor suppressors. Nat Chem Biol, 2011. 7(5): p. 285-95. DOI: 10.1038/nchembio.546

219. Silva JL, De Moura Gallo CV, Costa DC, and Rangel LP, Prion-like aggregation of mutant p53 in cancer. Trends Biochem Sci, 2014. 39(6): p. 260-7. DOI: 10.1016/j.tibs.2014.04.001

220. de Oliveira GAP, Petronilho EC, Pedrote MM, Marques MA, Vieira T, et al., The Status of p53 Oligomeric and Aggregation States in Cancer. Biomolecules, 2020. 10(4). DOI: 10.3390/biom10040548

221. Oren M and Rotter V, Mutant p53 gain-of-function in cancer. Cold Spring Harb Perspect Biol, 2010. 2(2): p. a001107. DOI: 10.1101/cshperspect.a001107

222. Mantovani F, Collavin L, and Del Sal G, Mutant $p 53$ as a guardian of the cancer cell. Cell Death Differ, 2019. 26(2): p. 199-212. DOI: 10.1038/s41418-018-02469

223. Bellazzo A, Sicari D, Valentino E, Del Sal G, and Collavin L, Complexes formed by mutant p53 and their roles in breast cancer. Breast Cancer (Dove Med Press), 2018. 10: p. 101-112. DOI: 10.2147/BCTT.S145826

224. Kim MP and Lozano G, Mutant p53 partners in crime. Cell Death Differ, 2018. 25(1): p. 161-168. DOI: 10.1038/cdd.2017.185

225. Liu X, Wilcken R, Joerger AC, Chuckowree IS, Amin J, et al., Small molecule induced reactivation of mutant p53 in cancer cells. Nucleic Acids Res, 2013. 41(12): p. 6034-44. DOI: 10.1093/nar/gkt305 
226. Schulz-Heddergott $\mathrm{R}$ and Moll UM, Gain-of-Function (GOF) Mutant p53 as Actionable Therapeutic Target. Cancers (Basel), 2018. 10(6). DOI: 10.3390/cancers10060188

227. Bykov VJ, Issaeva N, Shilov A, Hultcrantz M, Pugacheva E, et al., Restoration of the tumor suppressor function to mutant p53 by a low-molecular-weight compound. Nat Med, 2002. 8(3): p. 282-8. DOI: 10.1038/nm0302-282

228. Soragni A, Janzen DM, Johnson LM, Lindgren AG, Thai-Quynh Nguyen A, et al., A Designed Inhibitor of p53 Aggregation Rescues p53 Tumor Suppression in Ovarian Carcinomas. Cancer Cell, 2016. 29(1): p. 90-103. DOI: 10.1016/j.ccell.2015.12.002

229. Muller P, Hrstka R, Coomber D, Lane DP, and Vojtesek B, Chaperonedependent stabilization and degradation of p53 mutants. Oncogene, 2008. 27(24): p. 3371-83. DOI: 10.1038/sj.onc. 1211010

230. Xu J, Wang J, Hu Y, Qian J, Xu B, et al., Unequal prognostic potentials of $p 53$ gain-of-function mutations in human cancers associate with drug-metabolizing activity. Cell Death Dis, 2014. 5: p. e1108. DOI: 10.1038/cddis.2014.75

231. Graziano F and Cascinu S, Prognostic molecular markers for planning adjuvant chemotherapy trials in Dukes' B colorectal cancer patients: how much evidence is enough? Ann Oncol, 2003. 14(7): p. 1026-38. DOI: 10.1093/annonc/mdg284

232. Roman-Rosales AA, Garcia-Villa E, Herrera LA, Gariglio P, and Diaz-Chavez J, Mutant p53 gain of function induces HER2 over-expression in cancer cells. BMC Cancer, 2018. 18(1): p. 709. DOI: 10.1186/s12885-018-4613-1

233. Ahn JH, Kim TJ, Lee $\mathrm{JH}$, and Choi $\mathrm{JH}$, Mutant $p 53$ stimulates cell invasion through an interaction with Rad21 in human ovarian cancer cells. Sci Rep, 2017. 7(1): p. 9076. DOI: 10.1038/s41598-017-08880-4

234. Klemke L, De Oliveira T, Witt D, Winkler N, Bohnenberger H, et al., Hsp90stabilized MIF supports tumor progression via macrophage recruitment and angiogenesis in colorectal cancer. Cell Death Dis, 2021. 12(2): p. 155. DOI: 10.1038/s41419-021-03426-z

235. Schulz-Heddergott R and Moll UM, HSP90-Stabilized MIF in Oncogenesis and Cell Growth Control, in MIF Family Cytokines in Innate Immunity and Homeostasis, R. Bucala and J. Bernhagen, Editors. 2017, Springer International Publishing: Cham. p. 21-42. ISBN: 978-3-319-52354-5

236. Nishihira J, Ishibashi T, Fukushima T, Sun B, Sato Y, et al., Macrophage migration inhibitory factor (MIF): Its potential role in tumor growth and tumorassociated angiogenesis. Ann N Y Acad Sci, 2003. 995: p. 171-82. DOI: 10.1111/j.1749-6632.2003.tb03220.x

237. Fan H, Hall P, Santos LL, Gregory JL, Fingerle-Rowson G, et al., Macrophage migration inhibitory factor and CD74 regulate macrophage chemotactic responses via MAPK and Rho GTPase. J Immunol, 2011. 186(8): p. 4915-24. DOI: 10.4049/jimmunol.1003713

238. White ES, Strom SR, Wys NL, and Arenberg DA, Non-small cell lung cancer cells induce monocytes to increase expression of angiogenic activity. J Immunol, 2001. 166(12): p. 7549-55. DOI: 10.4049/jimmunol.166.12.7549 
239. Chesney JA and Mitchell RA, 25 Years On: A Retrospective on Migration Inhibitory Factor in Tumor Angiogenesis. Mol Med, 2015. 21 Suppl 1: p. S1924. DOI: 10.2119/molmed.2015.00055

240. Kanzler I, Tuchscheerer N, Steffens G, Simsekyilmaz S, Konschalla S, et al., Differential roles of angiogenic chemokines in endothelial progenitor cellinduced angiogenesis. Basic Res Cardiol, 2013. 108(1): p. 310. DOI: 10.1007/s00395-012-0310-4

241. Cui J, Zhang F, Wang Y, Liu J, Ming X, et al., Macrophage migration inhibitory factor promotes cardiac stem cell proliferation and endothelial differentiation through the activation of the PI3K/Akt/mTOR and AMPK pathways. Int J Mol Med, 2016. 37(5): p. 1299-309. DOI: 10.3892/ijmm.2016.2542

242. Girard E, Strathdee C, Trueblood E, and Queva C, Macrophage migration inhibitory factor produced by the tumour stroma but not by tumour cells regulates angiogenesis in the B16-F10 melanoma model. Br J Cancer, 2012. 107(9): p. 1498-505. DOI: 10.1038/bjc.2012.392

243. Weber C, Kraemer S, Drechsler M, Lue H, Koenen RR, et al., Structural determinants of MIF functions in CXCR2-mediated inflammatory and atherogenic leukocyte recruitment. Proc Natl Acad Sci U S A, 2008. 105(42): p. 16278-83. DOI: 10.1073/pnas.0804017105

244. Martin C, Burdon PC, Bridger G, Gutierrez-Ramos JC, Williams TJ, et al., Chemokines acting via CXCR2 and CXCR4 control the release of neutrophils from the bone marrow and their return following senescence. Immunity, 2003. 19(4): p. 583-93. DOI: 10.1016/s1074-7613(03)00263-2

245. Kruger P, Saffarzadeh M, Weber AN, Rieber N, Radsak M, et al., Neutrophils: Between host defence, immune modulation, and tissue injury. PLoS Pathog, 2015. 11(3): p. e1004651. DOI: 10.1371/journal.ppat.1004651

246. Hu CT, Guo LL, Feng N, Zhang L, Zhou N, et al., MIF, secreted by human hepatic sinusoidal endothelial cells, promotes chemotaxis and outgrowth of colorectal cancer in liver prometastasis. Oncotarget, 2015. 6(26): p. 22410-23. DOI: 10.18632/oncotarget.4198

247. el Marjou F, Janssen KP, Chang BH, Li M, Hindie V, et al., Tissue-specific and inducible Cre-mediated recombination in the gut epithelium. Genesis, 2004. 39(3): p. 186-93. DOI: 10.1002/gene.20042

248. Kim H, Kim M, Im SK, and Fang S, Mouse Cre-LoxP system: general principles to determine tissue-specific roles of target genes. Lab Anim Res, 2018. 34(4): p. 147-159. DOI: 10.5625/lar.2018.34.4.147

249. Djudjaj S, Martin IV, Buhl EM, Nothofer NJ, Leng L, et al., Macrophage Migration Inhibitory Factor Limits Renal Inflammation and Fibrosis by Counteracting Tubular Cell Cycle Arrest. J Am Soc Nephrol, 2017. 28(12): p. 3590-3604. DOI: 10.1681/ASN.2017020190

250. Brocks T, Fedorchenko O, Schliermann N, Stein A, Moll UM, et al., Macrophage migration inhibitory factor protects from nonmelanoma epidermal tumors by regulating the number of antigen-presenting cells in skin. FASEB J, 2017. 31(2): p. 526-543. DOI: $10.1096 / \mathrm{fj} .201600860 \mathrm{R}$

251. McClelland M, Zhao L, Carskadon S, and Arenberg D, Expression of CD74, the receptor for macrophage migration inhibitory factor, in non-small cell lung 
cancer. Am J Pathol, 2009. 174(2): p. 638-46. DOI: 10.2353/ajpath.2009.080463

252. Binsky I, Haran M, Starlets D, Gore Y, Lantner F, et al., IL-8 secreted in a macrophage migration-inhibitory factor- and CD74-dependent manner regulates B cell chronic lymphocytic leukemia survival. Proc Natl Acad Sci U S A, 2007. 104(33): p. 13408-13. DOI: 10.1073/pnas.0701553104

253. Wynn TA and Vannella KM, Macrophages in Tissue Repair, Regeneration, and Fibrosis. Immunity, 2016. 44(3): p. 450-462. DOI: 10.1016/j.immuni.2016.02.015

254. Wang J, Neutrophils in tissue injury and repair. Cell Tissue Res, 2018. 371(3): p. 531-539. DOI: 10.1007/s00441-017-2785-7

255. Wilgus TA, Roy S, and McDaniel JC, Neutrophils and Wound Repair: Positive Actions and Negative Reactions. Adv Wound Care (New Rochelle), 2013. 2(7): p. 379-388. DOI: 10.1089/wound.2012.0383

256. Li J, Tan J, Martino MM, and Lui KO, Regulatory T-Cells: Potential Regulator of Tissue Repair and Regeneration. Front Immunol, 2018. 9: p. 585. DOI: 10.3389/fimmu.2018.00585

257. Liu Y, Wang L, Kikuiri T, Akiyama K, Chen C, et al., Mesenchymal stem cellbased tissue regeneration is governed by recipient $T$ lymphocytes via IFNgamma and TNF-alpha. Nat Med, 2011. 17(12): p. 1594-601. DOI: $10.1038 / \mathrm{nm} .2542$

258. Pellowe AS, Sauler M, Hou Y, Merola J, Liu R, et al., Endothelial cell-secreted MIF reduces pericyte contractility and enhances neutrophil extravasation. FASEB J, 2019. 33(2): p. 2171-2186. DOI: 10.1096/fj.201800480R

259. Pantouris G, Syed MA, Fan C, Rajasekaran D, Cho TY, et al., An Analysis of MIF Structural Features that Control Functional Activation of CD74. Chem Biol, 2015. 22(9): p. 1197-205. DOI: 10.1016/j.chembiol.2015.08.006

260. Farr L, Ghosh S, Jiang N, Watanabe K, Parlak M, et al., CD74 Signaling Links Inflammation to Intestinal Epithelial Cell Regeneration and Promotes Mucosal Healing. Cell Mol Gastroenterol Hepatol, 2020. 10(1): p. 101-112. DOI: 10.1016/j.jcmgh.2020.01.009

261. Baugh JA, Gantier M, Li L, Byrne A, Buckley A, et al., Dual regulation of macrophage migration inhibitory factor (MIF) expression in hypoxia by CREB and HIF-1. Biochem Biophys Res Commun, 2006. 347(4): p. 895-903. DOI: 10.1016/j.bbrc.2006.06.148

262. Cheng B, Wang Q, Song Y, Liu Y, Liu Y, et al., MIF inhibitor, ISO-1, attenuates human pancreatic cancer cell proliferation, migration and invasion in vitro, and suppresses xenograft tumour growth in vivo. Sci Rep, 2020. 10(1): p. 6741. DOI: 10.1038/s41598-020-63778-y

263. Meyer-Siegler KL, Iczkowski KA, Leng L, Bucala R, and Vera PL, Inhibition of macrophage migration inhibitory factor or its receptor (CD74) attenuates growth and invasion of DU-145 prostate cancer cells. J Immunol, 2006. 177(12): p. 8730-9. DOI: 10.4049/jimmunol.177.12.8730

264. Haran M, Mirkin V, Braester A, Harpaz N, Shevetz O, et al., A phase I-Il clinical trial of the anti-CD74 monoclonal antibody milatuzumab in frail patients with 
refractory chronic lymphocytic leukaemia: A patient based approach. $\mathrm{Br} \mathrm{J}$ Haematol, 2018. 182(1): p. 125-128. DOI: 10.1111/bjh.14726

265. Mahalingam D, Patel MR, Sachdev JC, Hart LL, Halama N, et al., Phase I study of imalumab (BAX69), a fully human recombinant antioxidized macrophage migration inhibitory factor antibody in advanced solid tumours. $\mathrm{Br} \mathrm{J}$ Clin Pharmacol, 2020. 86(9): p. 1836-1848. DOI: 10.1111/bcp.14289

266. Schinagl A, Thiele M, Douillard P, Volkel D, Kenner L, et al., Oxidized macrophage migration inhibitory factor is a potential new tissue marker and drug target in cancer. Oncotarget, 2016. 7(45): p. 73486-73496. DOI: 10.18632/oncotarget.11970

267. Schindler L, Dickerhof N, Hampton MB, and Bernhagen J, Post-translational regulation of macrophage migration inhibitory factor: Basis for functional finetuning. Redox Biol, 2018. 15: p. 135-142. DOI: 10.1016/j.redox.2017.11.028

268. Thiele M, Kerschbaumer RJ, Tam FW, Volkel D, Douillard P, et al., Selective Targeting of a Disease-Related Conformational Isoform of Macrophage Migration Inhibitory Factor Ameliorates Inflammatory Conditions. J Immunol, 2015. 195(5): p. 2343-52. DOI: 10.4049/jimmunol.1500572

269. Sparkes A, De Baetselier P, Brys L, Cabrito I, Sterckx YG, et al., Novel half-life extended anti-MIF nanobodies protect against endotoxic shock. FASEB J, 2018. 32(6): p. 3411-3422. DOI: 10.1096/fj.201701189R

270. Harmsen MM and De Haard HJ, Properties, production, and applications of camelid single-domain antibody fragments. Appl Microbiol Biotechnol, 2007. 77(1): p. 13-22. DOI: 10.1007/s00253-007-1142-2

271. Sur S, Pagliarini R, Bunz F, Rago C, Diaz LA, Jr., et al., A panel of isogenic human cancer cells suggests a therapeutic approach for cancers with inactivated p53. Proc Natl Acad Sci U S A, 2009. 106(10): p. 3964-9. DOI: 10.1073/pnas.0813333106

272. Antonova E, Glazova O, Gaponova A, Eremyan A, Zvereva S, et al., Successful CRISPR/Cas9 mediated homologous recombination in a chicken cell line. F1000Res, 2018. 7: p. 238. DOI: 10.12688/f1000research.13457.2

273. Vartanian S, Bentley C, Brauer MJ, Li L, Shirasawa S, et al., Identification of mutant K-Ras-dependent phenotypes using a panel of isogenic cell lines. J Biol Chem, 2013. 288(4): p. 2403-13. DOI: 10.1074/jbc.M112.394130

274. Igelmann S, Neubauer HA, and Ferbeyre G, STAT3 and STAT5 Activation in Solid Cancers. Cancers (Basel), 2019. 11(10). DOI: 10.3390/cancers11101428

275. Wu CJ, Sundararajan V, Sheu BC, Huang RY, and Wei LH, Activation of STAT3 and STAT5 Signaling in Epithelial Ovarian Cancer Progression: Mechanism and Therapeutic Opportunity. Cancers (Basel), 2019. 12(1). DOI: 10.3390/cancers12010024

276. Lee H, Herrmann A, Deng JH, Kujawski M, Niu G, et al., Persistently activated Stat3 maintains constitutive NF-kappaB activity in tumors. Cancer Cell, 2009. 15(4): p. 283-93. DOI: 10.1016/j.ccr.2009.02.015

277. Baumgart S, Chen NM, Siveke JT, Konig A, Zhang JS, et al., Inflammationinduced NFATC1-STAT3 transcription complex promotes pancreatic cancer 
initiation by KrasG12D. Cancer Discov, 2014. 4(6): p. 688-701. DOI: 10.1158/2159-8290.CD-13-0593

278. Qin JJ, Yan L, Zhang J, and Zhang WD, STAT3 as a potential therapeutic target in triple negative breast cancer: a systematic review. J Exp Clin Cancer Res, 2019. 38(1): p. 195. DOI: 10.1186/s13046-019-1206-z

279. Zou S, Tong Q, Liu B, Huang W, Tian Y, et al., Targeting STAT3 in Cancer Immunotherapy. Mol Cancer, 2020. 19(1): p. 145. DOI: 10.1186/s12943-02001258-7

280. Siveen KS, Sikka S, Surana R, Dai X, Zhang J, et al., Targeting the STAT3 signaling pathway in cancer: role of synthetic and natural inhibitors. Biochim Biophys Acta, 2014. 1845(2): p. 136-54. DOI: 10.1016/j.bbcan.2013.12.005

281. Cooks T, Pateras IS, Tarcic O, Solomon H, Schetter AJ, et al., Mutant p53 prolongs NF-kappaB activation and promotes chronic inflammation and inflammation-associated colorectal cancer. Cancer Cell, 2013. 23(5): p. 634-46. DOI: 10.1016/j.ccr.2013.03.022

282. Weisz L, Damalas A, Liontos M, Karakaidos P, Fontemaggi G, et al., Mutant p53 enhances nuclear factor kappaB activation by tumor necrosis factor alpha in cancer cells. Cancer Res, 2007. 67(6): p. 2396-401. DOI: 10.1158/00085472.CAN-06-2425

283. Schneider $\mathrm{G}$, Henrich $\mathrm{A}$, Greiner $\mathrm{G}$, Wolf $\mathrm{V}$, Lovas $\mathrm{A}$, et al., Cross talk between stimulated NF-kappaB and the tumor suppressor p53. Oncogene, 2010. 29(19): p. 2795-806. DOI: 10.1038/onc.2010.46

284. Dell'Orso S, Fontemaggi G, Stambolsky P, Goeman F, Voellenkle C, et al., ChIP-on-chip analysis of in vivo mutant $p 53$ binding to selected gene promoters. OMICS, 2011. 15(5): p. 305-12. DOI: 10.1089/omi.2010.0084

285. Hanel W, Marchenko N, Xu S, Yu SX, Weng W, et al., Two hot spot mutant p53 mouse models display differential gain of function in tumorigenesis. Cell Death Differ, 2013. 20(7): p. 898-909. DOI: 10.1038/cdd.2013.17

286. Landmann $\mathrm{H}$, Proia DA, He S, Ogawa LS, Kramer $F$, et al., UDP glucuronosyltransferase $1 \mathrm{~A}$ expression levels determine the response of colorectal cancer cells to the heat shock protein 90 inhibitor ganetespib. Cell Death Dis, 2014. 5: p. e1411. DOI: 10.1038/cddis.2014.378

287. Angstadt AY, Hartman TJ, Lesko SM, Muscat JE, Zhu J, et al., The effect of UGT1A and UGT2B polymorphisms on colorectal cancer risk: haplotype associations and gene-environment interactions. Genes Chromosomes Cancer, 2014. 53(6): p. 454-66. DOI: 10.1002/gcc.22157

288. Li D, Marchenko ND, and Moll UM, SAHA shows preferential cytotoxicity in mutant p53 cancer cells by destabilizing mutant p53 through inhibition of the HDAC6-Hsp90 chaperone axis. Cell Death Differ, 2011. 18(12): p. 1904-13. DOI: $10.1038 /$ cdd. 2011.71

289. Parrales $\mathrm{A}$, Thoenen $\mathrm{E}$, and Iwakuma $\mathrm{T}$, The interplay between mutant $\mathrm{p} 53$ and the mevalonate pathway. Cell Death Differ, 2018. 25(3): p. 460-470. DOI: 10.1038/s41418-017-0026-y 
290. Parrales A, Ranjan A, lyer SV, Padhye S, Weir SJ, et al., DNAJA1 controls the fate of misfolded mutant p53 through the mevalonate pathway. Nat Cell Biol, 2016. 18(11): p. 1233-1243. DOI: $10.1038 / n c b 3427$

291. Silva JL, Cino EA, Soares IN, Ferreira VF, and G APdO, Targeting the Prionlike Aggregation of Mutant p53 to Combat Cancer. Acc Chem Res, 2018. 51(1): p. 181-190. DOI: 10.1021/acs.accounts.7b00473

292. Zhang Y, Coillie SV, Fang JY, and Xu J, Gain of function of mutant $p 53:$ R282W on the peak? Oncogenesis, 2016. 5: p. e196. DOI: 10.1038/oncsis.2016.8

293. Kehrloesser S, Osterburg C, Tuppi M, Schafer B, Vousden $\mathrm{KH}$, et al., Intrinsic aggregation propensity of the p63 and p73 TI domains correlates with p53R175H interaction and suggests further significance of aggregation events in the p53 family. Cell Death Differ, 2016. 23(12): p. 1952-1960. DOI: 10.1038/cdd.2016.75

294. Yue X, Zhao Y, Xu Y, Zheng M, Feng Z, et al., Mutant p53 in Cancer: Accumulation, Gain-of-Function, and Therapy. J Mol Biol, 2017. 429(11): p. 1595-1606. DOI: 10.1016/j.jmb.2017.03.030

295. Kolosenko I, Yu Y, Busker S, Dyczynski M, Liu J, et al., Identification of novel small molecules that inhibit STAT3-dependent transcription and function. PLoS One, 2017. 12(6): p. e0178844. DOI: 10.1371/journal.pone.0178844

296. Debnath B, Xu S, and Neamati N, Small molecule inhibitors of signal transducer and activator of transcription 3 (Stat3) protein. J Med Chem, 2012. 55(15): p. 6645-68. DOI: 10.1021/jm300207s

297. Spitzner M, Roesler B, Bielfeld C, Emons G, Gaedcke J, et al., STAT3 inhibition sensitizes colorectal cancer to chemoradiotherapy in vitro and in vivo. Int $\mathrm{J}$ Cancer, 2014. 134(4): p. 997-1007. DOI: 10.1002/ijc.28429

298. Kawazoe A, Kuboki Y, Bando H, Fukuoka S, Kojima T, et al., Phase 1 study of napabucasin, a cancer stemness inhibitor, in patients with advanced solid tumors. Cancer Chemother Pharmacol, 2020. 85(5): p. 855-862. DOI: 10.1007/s00280-020-04059-3

299. Boston Biomedical, Inc., Boston Biomedical Announces Orphan Drug Designation for Napabucasin in Pancreatic Cancer. 2016 [cited 20.04.2021]; Available from: https://www.prnewswire.com/news-releases/bostonbiomedical-announces-orphan-drug-designation-for-napabucasin-inpancreatic-cancer-300361932.html.

300. Boston Biomedical, Inc., Boston Biomedical, Inc. Announces Update on Phase 3 CanStem111P Study of Napabucasin in Patients with Metastatic Pancreatic Cancer Following Interim Analysis. 2019 [cited 20.04.2021]; Available from: https://www.prnewswire.com/news-releases/boston-biomedical-incannounces-update-on-phase-3-canstem111p-study-of-napabucasin-inpatients-with-metastatic-pancreatic-cancer-following-interim-analysis300879064.html.

301. Ling X, Konopleva M, Zeng Z, Ruvolo V, Stephens LC, et al., The novel triterpenoid C-28 methyl ester of 2-cyano-3, 12-dioxoolen-1, 9-dien-28-oic acid inhibits metastatic murine breast tumor growth through inactivation of STAT3 signaling. Cancer Res, 2007. 67(9): p. 4210-8. DOI: 10.1158/0008-5472.CAN06-3629 
302. Duan Z, Ames RY, Ryan M, Hornicek FJ, Mankin H, et al., CDDO-Me, a synthetic triterpenoid, inhibits expression of IL-6 and Stat3 phosphorylation in multi-drug resistant ovarian cancer cells. Cancer Chemother Pharmacol, 2009. 63(4): p. 681-9. DOI: 10.1007/s00280-008-0785-8

303. Hong DS, Kurzrock R, Supko JG, He X, Naing A, et al., A phase I first-in-human trial of bardoxolone methyl in patients with advanced solid tumors and lymphomas. Clin Cancer Res, 2012. 18(12): p. 3396-406. DOI: 10.1158/10780432.CCR-11-2703

304. Ahmad R, Raina D, Meyer C, Kharbanda S, and Kufe D, Triterpenoid CDDOMe blocks the NF-kappaB pathway by direct inhibition of IKKbeta on Cys-179. J Biol Chem, 2006. 281(47): p. 35764-9. DOI: 10.1074/jbc.M607160200

305. Capaci V, Mantovani F, and Del Sal G, Amplifying Tumor-Stroma Communication: An Emerging Oncogenic Function of Mutant p53. Front Oncol, 2020. 10: p. 614230. DOI: $10.3389 /$ fonc.2020.614230

306. Stein $\mathrm{Y}$, Aloni-Grinstein R, and Rotter V, Mutant p53-a potential player in shaping the tumor-stroma crosstalk. J Mol Cell Biol, 2019. 11(7): p. 600-604. DOI: 10.1093/jmcb/mjz071

307. Pavlakis E and Stiewe T, p53's Extended Reach: The Mutant p53 Secretome. Biomolecules, 2020. 10(2). DOI: 10.3390/biom10020307

308. Butera G, Brandi J, Cavallini C, Scarpa A, Lawlor RT, et al., The Mutant p53Driven Secretome Has Oncogenic Functions in Pancreatic Ductal Adenocarcinoma Cells. Biomolecules, 2020. 10(6). DOI: 10.3390/biom10060884

309. Sperb N, Tsesmelis M, and Wirth T, Crosstalk between Tumor and Stromal Cells in Pancreatic Ductal Adenocarcinoma. Int J Mol Sci, 2020. 21(15). DOI: 10.3390/ijms21155486

310. Garcia PL, Miller AL, and Yoon KJ, Patient-Derived Xenograft Models of Pancreatic Cancer: Overview and Comparison with Other Types of Models. Cancers (Basel), 2020. 12(5). DOI: 10.3390/cancers12051327

311. Cooks T, Pateras IS, Jenkins LM, Patel KM, Robles AI, et al., Mutant p53 cancers reprogram macrophages to tumor supporting macrophages via exosomal miR-1246. Nat Commun, 2018. 9(1): p. 771. DOI: 10.1038/s41467018-03224-w

312. Wang D, Wang R, Huang A, Fang Z, Wang K, et al., Upregulation of macrophage migration inhibitory factor promotes tumor metastasis and correlates with poor prognosis of pancreatic ductal adenocarcinoma. Oncol Rep, 2018. 40(5): p. 2628-2636. DOI: 10.3892/or.2018.6703

313. Yang S, He P, Wang J, Schetter A, Tang W, et al., A Novel MIF Signaling Pathway Drives the Malignant Character of Pancreatic Cancer by Targeting NR3C2. Cancer Res, 2016. 76(13): p. 3838-50. DOI: 10.1158/0008-5472.CAN$15-2841$

314. Funamizu N, Hu C, Lacy C, Schetter A, Zhang G, et al., Macrophage migration inhibitory factor induces epithelial to mesenchymal transition, enhances tumor aggressiveness and predicts clinical outcome in resected pancreatic ductal adenocarcinoma. Int J Cancer, 2013. 132(4): p. 785-94. DOI: 10.1002/ijc.27736 


\section{ACKNOWLEDGEMENTS}

Starting my $\mathrm{PhD}$ in 2017, I can now look back on 3.5 years of constructive collaborations, great experiences and lots of memories. In this regard I would like to express my special thanks to those who accompanied me on my way:

To begin with, I would like to thank my supervisor PD Dr. Ramona Schulz-Heddergott for the great opportunity to work in her group during my PhD. I am very thankful for her constant support, insightful feedback and guidance throughout my projects. Her commitment for the team and for science is inspiring.

I also take this opportunity, to thank Prof. Matthias Dobbelstein for giving me the opportunity to be part of his Institute of Molecular Oncology. I am grateful for the valuable input and advice I got during our regular jour fixes and seminars.

Furthermore, I would like to extend my gratitude to my TAC members Prof. Holger Reichardt and Prof. Argyris Papantonis for fruitful scientific discussions during the thesis advisory committee meetings. Additionally, I thank Prof. Heidi Hahn, Dr. Shiv Singh and Dr. Nico Posnien for being members of the extended examination board.

Many thanks also to all past and present members of the $3^{\text {rd }}$ floor of GZMB and especially the Molecular Oncology for generating such a cheerful, productive and interactive working environment. In particular I would like to thank the best PhD buddy I could imagine: Valentina Manzini. From the very beginning, we were going through all phases of everyday $\mathrm{PhD}$ life together. I am very grateful for her constant encouragement, our vivid discussions and the friendship we have. I would also like to thank Dr. Josephine Choo for always giving great advice throughout my $\mathrm{PhD}$ and for spreading joy in the lab. Furthermore, I would like to thank Nadine Winkler and Tamara Isermann for their constant support, our collaboration and the great team spirit we have had. I really appreciated the daily work with all members of the MolOnkol in the past couple of years. 
In addition, I thank all co-authors, collaborators and everyone who contributed to my projects. I would like to extend my gratitude to the Göttingen Graduate School for Neurosciences, Biophysics, and Molecular Biosciences (GGNB) and the program Molecular Medicine for giving me the chance of being part of the program and a great network.

Special thanks also to Valentina, Josephine and Matthias for taking the time to proofread my thesis.

Finally, I would like to express my gratitude for my family and friends. Thanks a lot for your never-ending encouragement and tremendous support throughout my way. 


\section{$7 \quad$ AFFIDAVIT}

Herewith I declare that the PhD Thesis entitled "HSP90-stabilized proteins as therapeutic targets in cancer" was written independently and with no other sources and aids than quoted.

Luisa Klemke

Göttingen, April 2021 